\title{
Late Givetian ammonoids from Hassi Nebech (Tafilalt Basin, Anti-Atlas, southern Morocco)
}

\author{
Jürgen Bockwinkel ${ }^{1}$, R. Thomas Becker ${ }^{*}, 2$ and $\dagger$ Volker Ebbighausen $^{3}$ \\ ${ }^{1}$ Dechant-Fein-Str. 22, 51375 Leverkusen, Germany. E-mail: jbockwinkel@t-online.de \\ ${ }^{2}$ Institut für Geologie und Paläontologie, WWU Münster, Corrensstr. 24, 48149 Münster, Germany. E-mail: rbecker@uni-muenster.de \\ ${ }^{3}$ deceased on 3 rd June 2011
}

Received 7 January 2012

Accepted 3 September 2012

Published 20 February 2013

\begin{abstract}
The Hassi Nebech area of the SE Tafilalt (Tafilalt Basin, Anti-Atlas, Morocco) yielded the richest and most diverse late Givetian ammonoid fauna on a global scale. Above the distinctive regional "Lower Marker Bed" (Synpharciceras clavilobum Zone), abundant loosely collected limonitic specimens derive from hypoxic shales of the Taouzites taouzensis to Petteroceras errans zones. The ontogenetic morphometry and intraspecific variability of a total of 30 species representing five families, the Acanthoclymeniidae, Taouzitidae, Pharciceratidae, Petteroceratidae, and Tornoceratidae, are documented. New taxa are: Pseudoprobeloceras praecox n. sp., Scaturites minutus n. gen. n. sp., Darkaoceras velox n. sp., Pharciceras decoratum n. sp., Ph. fornix n. sp., Ph. subconstans n. sp., Ph. involutum n. sp., Lunupharciceras incisum n. sp., Transpharciceras procedens $\mathrm{n}$. gen. n. sp., Stenopharciceras progressum n. sp., Pluripharciceras n. gen. (type species: Synpharciceras plurilobatum Petter, 1959), Plu. orbis n. sp., Synpharciceras frequens n. sp., Lobotornoceras bensaidi n. sp., Nebechoceras eccentricum n. gen. n. sp., and Phoenixites lenticulus $\mathrm{n}$. $\mathrm{sp}$.. The documentation of conch and particularly suture ontogeny and intraspecific variability necessitates a revised diagnosis for ten taxa. Manticoceras pontiformis Termier \& Termier, 1950, Probeloceras costulatum Petter, 1959, and Pseudoprobeloceras nebechense Bensaid, 1974 are regarded as subjective junior synonyms of $P$ s. pernai (Wedekind, 1918). Sandbergeroceras acutum Termier \& Termier, 1950 is a subjective synonym of Taouzites taouzensis (Termier \& Termier, 1950). Pharciceras applanatum Bensaï, 1974 is transferred to Extropharciceras. Other forms ( $P h$. aff. tridens, Ph. cf. subconstans n. sp., Extropharciceras n. sp. 2, Ex. cf. arenicum, Ex. cf. applanatum, Synpharciceras sp., Plu. cf. plurilobatum) are described in open nomenclature.
\end{abstract}

Devonian

Ammonoidea

Ontogeny

Systematics

Biostratigraphy

Additional supporting information may be found in the online version of this article at the publisher's web-site

\section{Introduction}

The Devonian succession of the eastern Anti-Atlas (Tafilalt and Maider) of southern Morocco has been celebrated in numerous publications for its open marine faunas, notably for its ammonoids. Such a study is of particular interest, because the late Givetian was a very peculiar time in ammonoid evolution (e.g., House 1985) because of the rapid complication of septa in separate lineages of the distinctive families Taouzitidae, Pharciceratidae, and Petteroceratidae. As outlined in our first contribution on the revision and description of
Moroccan pharciceratid faunas (Bockwinkel et al. 2009), the eastern Anti-Atlas is the best region on a global scale to investigate such assemblages, based both on the shear number of available, well-preserved specimens, and on the globally highest beta (regional) diversity. This earlier study dealt with the fauna from the "Lower Marker Bed" of Dar Kaoua, which is continued here by a detailed description of the limonitic fauna from overlying shales of the famous Hassi Nebech region in the more basinal setting of the south-eastern Tafilalt. More than 1,800 specimens have been investigated, which fall in 30 species. For comparison, there

\footnotetext{
* Corresponding author
} 


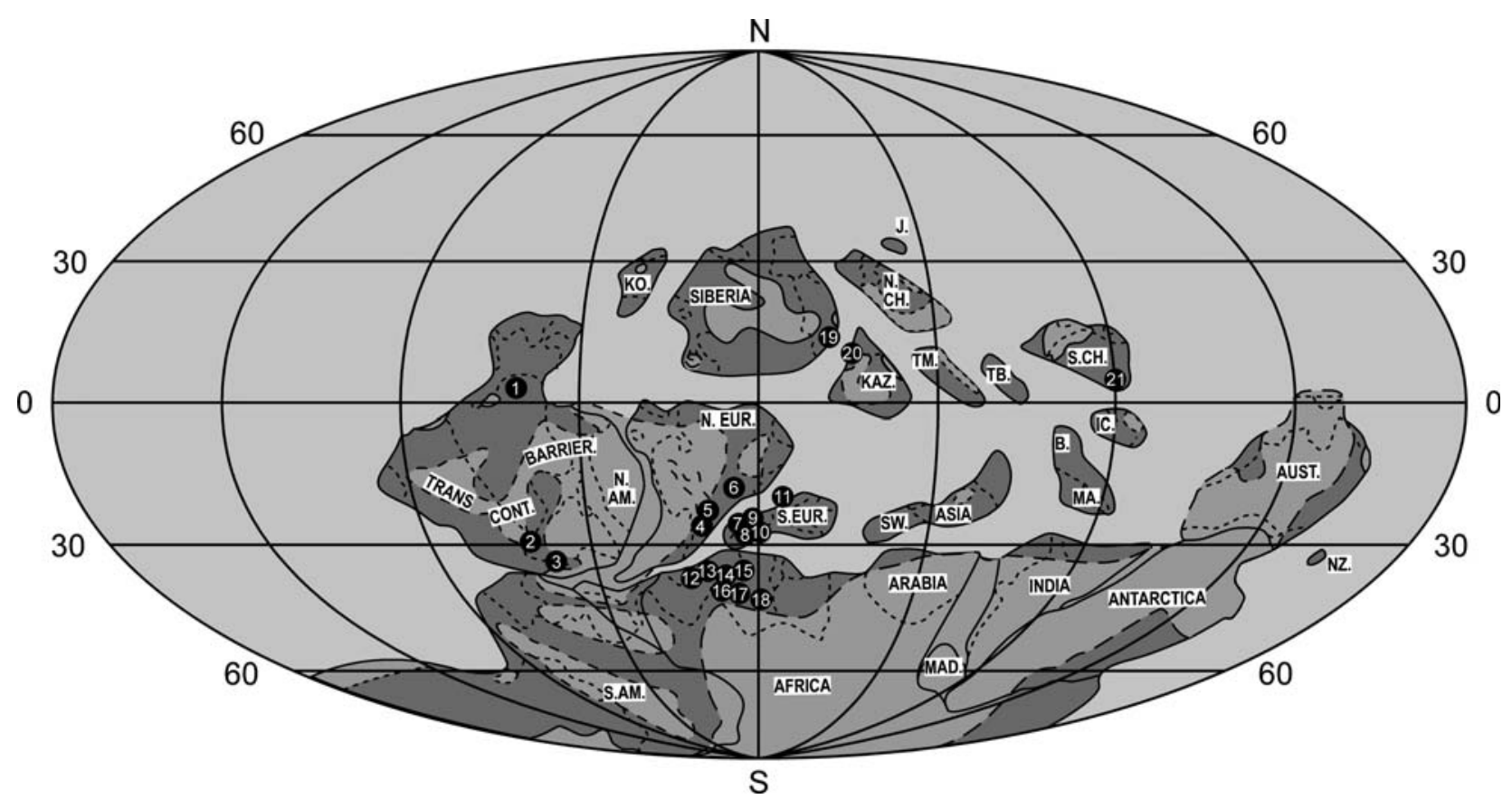

Figure 1. Global distribution of Late Givetian ammonoids. $1=$ NW Territorries, $2=$ Kentucky, $3=$ New York State, $4=$ Rhenish Massive, $5=$ Harz Mountains, $6=$ Sudetes, $7=$ Cantabrian Mountains, $8=$ Westiberian Chains (Celtiberia), $9=$ Pyrenees, $10=$ Montagne Noire, $11=$ Graz Palaeozoic, $12=$ eastern Dra Valley, $13=$ Maider, $14=$ Tafilalt, $15=$ Ben Zireg region, $16=$ Ougarta, $17=$ Saoura Valley, $18=$ Ahnet, $19=$ Rudny Altai, $20=$ Semipalatinsk region, eastern Kazakhstan, $21=$ Guangxi, South China. J. = Japan, KO. = Kolyma, N. EUR. = northern Europe, N. AM. = North America, S. AM. = South America, S. EUR. $=$ southern Europe, N. CH. = North China, KAZ. = Kazakhstan, TM. = Tarim Basin, TB. = Tibet, S. CH. = South China, IC. = Indochine, B. = Burma, MA. = Malyasia, AUST. = Australia, NZ. = New Zealand, MAD. = Madagascar. Plate tectonic reconstruction altered from Heckel \& Witzke (1979), preferred to other Devonian world reconstructions because of the placing of Asian plates.

are currently only ca. 20 named species for all of the late Givetian of the Rhenish Massive, the region with the next highest documented diversity.

For a brief review of the palaeogeographic distribution (Fig. 1) of pharciceratid faunas and of previous reports from the Anti-Atlas, the reader is referred to Bockwinkel et al. (2009). The late Givetian ammonoid zonation and conodont correlation within the Tafilalt region was summarized by Becker \& House (2000a, 2000b) and Aboussalam \& Becker (2007). The applied zonal key for correlation, with $\mathrm{MD}=$ Middle Devonian and $\mathrm{UD}=$ Upper Devonian, is also shown in the concise geological time scale of IUGS (Ogg et al. 2008). The late Givetian succession of the Tafilalt consists of the following ammonoid succession: MD III-B = Mzerrebites erraticus Zone; MD III-C = Synpharciceras clavilobum Zone; MD III-D = Taouzites taouzensis Zone; MD III-E = Petteroceras errans Zone.

\section{Locality and Stratigraphy}

The Hassi Nebech area in the south-eastern Tafilalt (Fig. 2) exposes an Early to Late Devonian succession in an elongated, ca. WNW-ESE striking band of low outcrop for several kilometres, starting ca. $19 \mathrm{~km}$ eastnortheast from Taouz. Following early records of pharciceratids in rather generalized faunal lists for the Er- foud region by Termier (1929) and Clariond (1934), it is the first North African locality from which a late Givetian goniatite was illustrated (Synpharciceras n. sp. aff. clavilobus in Schindewolf 1940, here included in Syn. clavilobum). Based on the Marcais and Clariond Collection, Termier \& Termier (1950) briefly described from the same series of outcrops, but then named Oued Kseir (which lies to the East) and Tazoult Nehra (a marker hill to the North), the following six species:

Koenenites lamellosus (= Mzerrebites sp. and Darkaoceras velox $\mathrm{n} . \mathrm{sp}$.

Pharciceras taouzensis (placed in Taouzites by Korn 2001; partly including Synpharciceras plurilobatum)

Pharciceras tridens (partly including an Extropharciceras)

Pharciceras tafilense (= Synpharciceras frequens n. sp.)

Pharciceras kseirense (placed in Stenopharciceras by Montesinos \& Henn 1986)

Pharciceras lunulicosta $(?=$ Synpharciceras frequens n. sp.)

Bensaï (1974) added 11 species from the main shale levels of the same locality.

Tornoceras simplex (= probably Nebechoceras eccentricum n. gen. n. sp.)

Tornoceras frechi (= partly Lobotornoceras bensaidi n. sp.) 


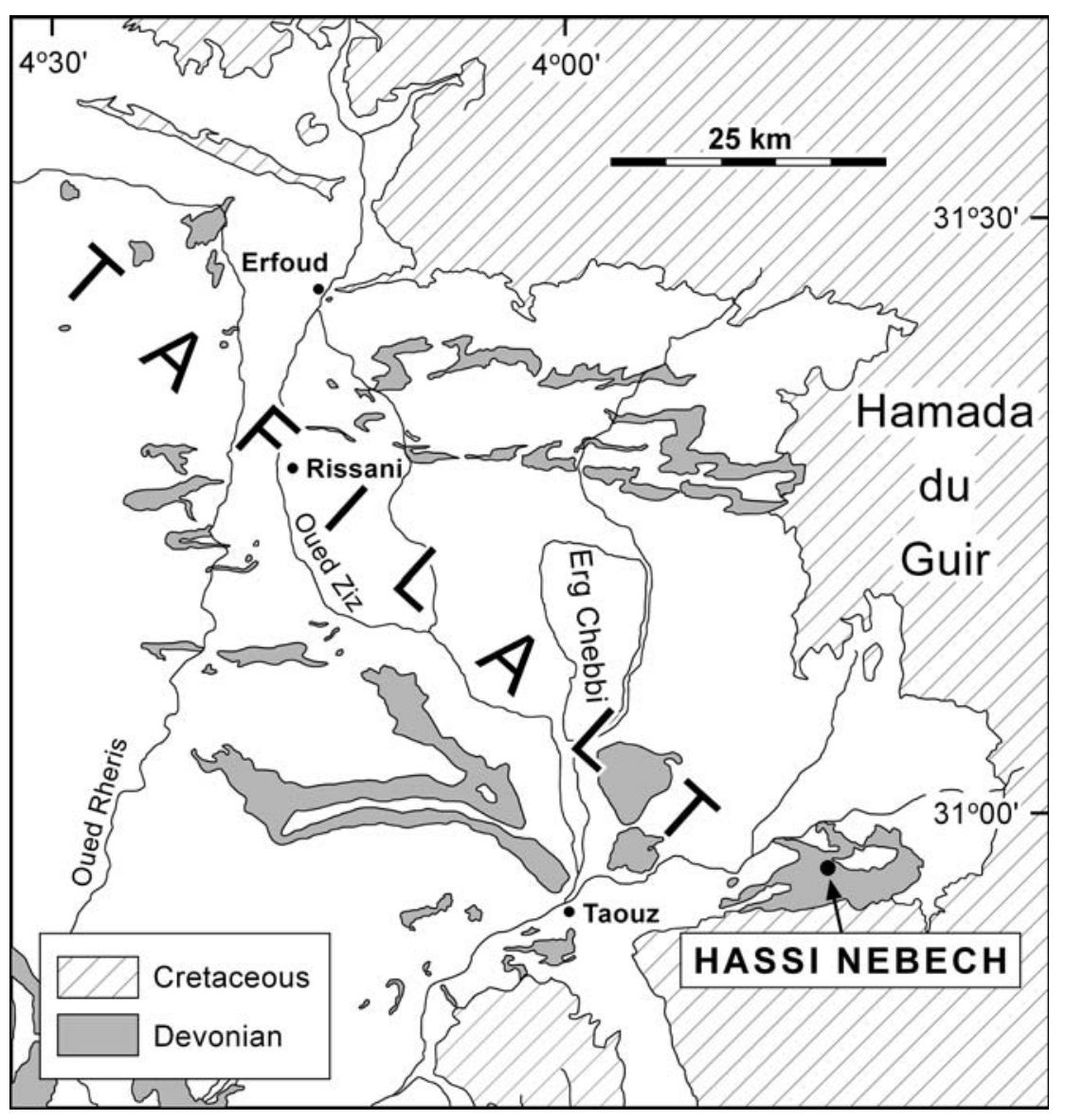

Figure 2. Location of the Hassi Nebech area in the SE Tafilalt (modified from Kaufmann 1998).
Pharciceras cf. meridionale (= Darkaoceras velox n. sp.)

Pharciceras applanatum (here re-assigned to Extropharciceras)

Pharciceras cf. applanatum (= Pharciceras fornix n. sp.)

Pharciceras n. sp. (e.p. = Stenopharciceras kseirense)

Synpharciceras clavilobum (= Syn. clavilobum, Syn. frequens n. sp., and Syn. aff. frequens n. sp.)

Synpharciceras aff. clavilobum (= Synpharciceras frequens n. sp.)

Beloceras? disciforme (placed in Meropharciceras by Becker \& House 1993)

Petteroceras errans

Petteroceras n. sp. (= Pett. errans)

Pseudoprobeloceras nebechense (= Pseudoprobeloceras pernai and Ps. praecox n. sp.)

Three Givetian-Frasnian sections have been measured since 1997, numbered 1a, 1b, and 2 (Becker \& House field numbering, see Aboussalam 2003). Details of Section 1a have not yet been published but topmost Middle Givetian nodular limestones yielded a new, very large-eyed phacopid in association with the oldest Pharciceras (Stegemann 2005). Above, deeply weathered shales are very rich in slightly abraded (rounded) limonitic and haematitic pharciceratids and other goniatites. This loose collection complements the main material from Section 2 described here. Section $1 \mathrm{~b}$ (at $\mathrm{N}$ $30^{\circ} 56^{\prime} 19^{\prime \prime}$, W $003^{\circ} 47^{\prime} 04^{\prime \prime}$ ), just 50 m east of Section 1a, has been investigated in detail by Aboussalam (2003) and does not reach the main pharciceratid levels. Nodular limestones at its top gently dip to the South and fall in the basal late Givetian Mzerrebites erraticus Zone (hermanni and cristatus ectypus conodont zones). Higher strata are covered by soil.

Section 2 is the succession that first was studied by Bensaïd (1974) and Bensaïd et al. (1985). It lies ca. $2 \mathrm{~km}$ east of Section $1 \mathrm{~b}$, at N $30^{\circ} 56^{\prime} 04^{\prime \prime}$, W $003^{\circ} 46^{\prime} 03^{\prime \prime}$, and exposes middle Givetian argillaceous beds with maenioceratids to Middle Frasnian nodular limestones with Sandbergeroceras and Naplesites, followed by Mesobeloceras, early Beloceras and Carinoceras. As outlined in Bensaïd et al. (1985), the sedimentary sequence indicates deeper deposition than in the Givetian/Frasnian boundary section in the central Tafilalt. This is consistent with assignment of the Hassi Nebech region to a Tafilalt Basin by Wendt et al. (1984) and Wendt (1988). Bensaïd et al. (1985) proposed that Section 2 could become the type-section of a formally defined Pharciceras Stage but the local conodont record is poor (Aboussalam \& Becker 2007; Gouwy et al. 2007). Aboussalam (2003) and Aboussalam \& Becker (2011) concentrated on the interval around the Taghanic Crisis level, starting in Middle Givetian shales and limestones. These are in places very rich in Maenioceras and Afromaenioceras, and overlain by the regional "Lower Marker Bed" (sensu Becker \& House 2000a and Bockwin- 


\section{Mesobeloceras}

white limestone with Manticoceras and Mesobeloceras

(lateral styliolinite)

(lateral siderite)

buff marly limestone

white limestone with Sandbergeroceras

red nodular limestone with Sandbergeroceras

styliolinite

septarians

septarians

siderite beds

turbiditic limestone

white limestone

white nodules

main collecting level

Lower Marker Bed

deeply weathered shale and marl

nodular micritic limestone

$\infty$ limestone nodules

番 marly septarians

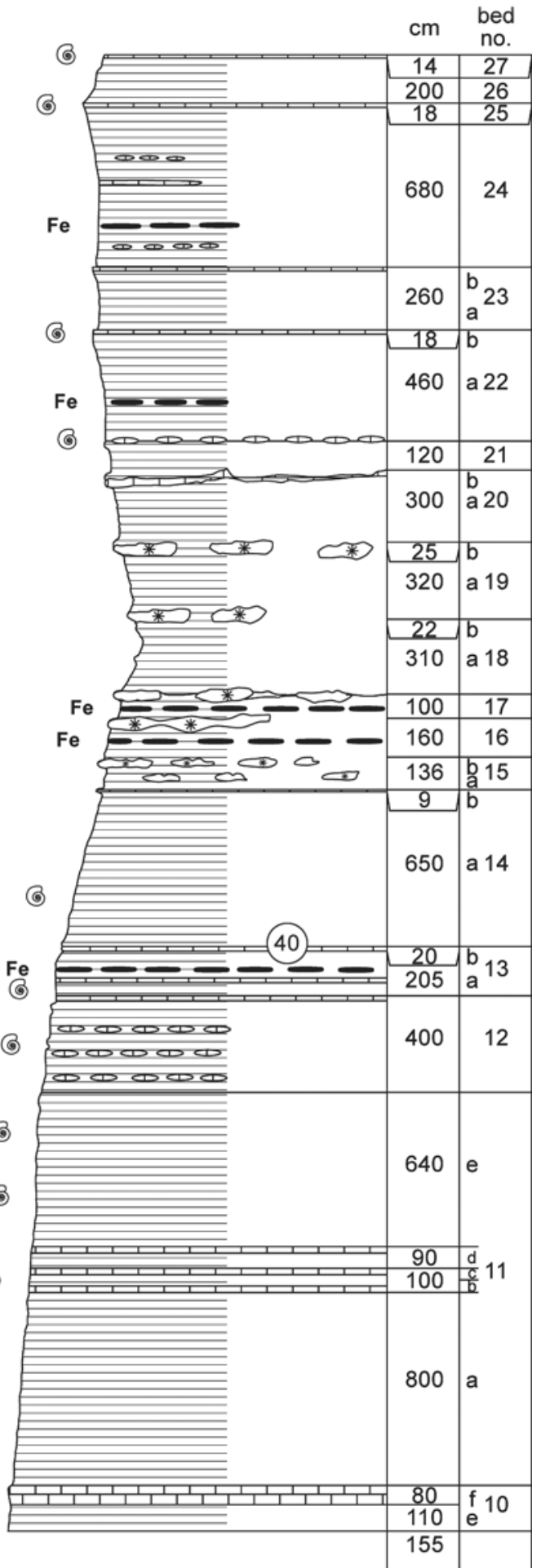

Fe brown siderite plates

(6) ammonoids

(40) number of Bensaid el. al. 1985
Figure 3. Lithological log of Hassi Nebech, Section 2, showing the position of ammonoid levels and marker units (based on joint work with the late M. R. House in 1997 and 1999, updated in spring 2011). kel et al. 2009) of the Synpharciceras clavilobum and disparilis zones. Our study concentrates on the richly fossiliferous overlying hypoxic shales that alternate with thin layers of nodular limestone, siderite and calcareous concretions. The goniatites were collected loose from the interval between the "Lower Marker Bed" (Bed 10f) and a second nodular limestone (Bed 13b) that forms the top of a very low, gentle slope. There is little contamination of faunas by transport during occasional flooding. Transported goniatites, especially those 
from section Hassi Nebech 1a to the West, are often somewhat polished and display a distinctive, abraded preservation. The sedimentary and faunal sequence at Section 2 correlates with the lower part of the Bouia Formation of the Tafilalt Platform (Hollard 1981). The investigated succession, (Section 2, Fig. 3) is summarized in the supplementary online material (1. Investigated succession), starting at the base with the numbered sequence of Aboussalam (2003).

\section{Material and methods}

All types and figured or numbered specimens from Hassi Nebech and material from Oberscheld used for comparison, except former specimens of Petter (1959) or of Bensaïd (1974) from the Bonn University collection STIPB-(old: GPI Bo)-Bensaïd numbers, are reposited in the collection of the Museum für Naturkunde, Berlin, under the numbers MB.C.22101.1-MB.C.22150; not described specimens remain in the Bockwinkel, Becker (WWU Münster), and Ebbighausen collections (MfN Berlin). The following genus abbreviations are used throughout the text: D. = Darkaoceras, Ep. = Epitornoceras, Ex. $=$ Extropharciceras, $L .=$ Lobotornoceras, Lu. $=$ Lunupharciceras, Me.$=$ Meropharciceras,$\quad M z .=$ Mzerrebites,$\quad N .=$ Nebechoceras n. gen., $\quad P .=$ Phoenixites, $\quad$ Pett. $=$ Petteroceras,$\quad P h .=$ Pharciceras Plu. $=$ Pluripharciceras n. gen., Ps. $=$ Pseudoprobeloceras, $S c .=$ Scaturites n. gen., St. = Stenopharciceras, Syn. = Synpharciceras, T. = Taouzites, Tr. = Transpharciceras n. gen. The abbreviations of conch parameters and suture elements are depicted in Figure 4. Plurilobes (Pl), irregular, often unilateral or episodic saddle incisions, are not recognized in suture formulae. Terminology and definitions of conch proportions follow Korn (2010).

For the understanding of evolutionary terms (parallel evolution, micromorphism) we refer to Landman (1988), Kennedy \& Cobban (1990), Skelton (1993), Marchand et al. (2002), and Monnet et al. (2011).

For synonymy lists, descriptions, discussions, and stratigraphic as well as geographic distribution of taxa, see supplementary online material (2. Systematic Palaeontology); for conch dimensions and ratios, see supplementary online material (3. Conch dimensions).

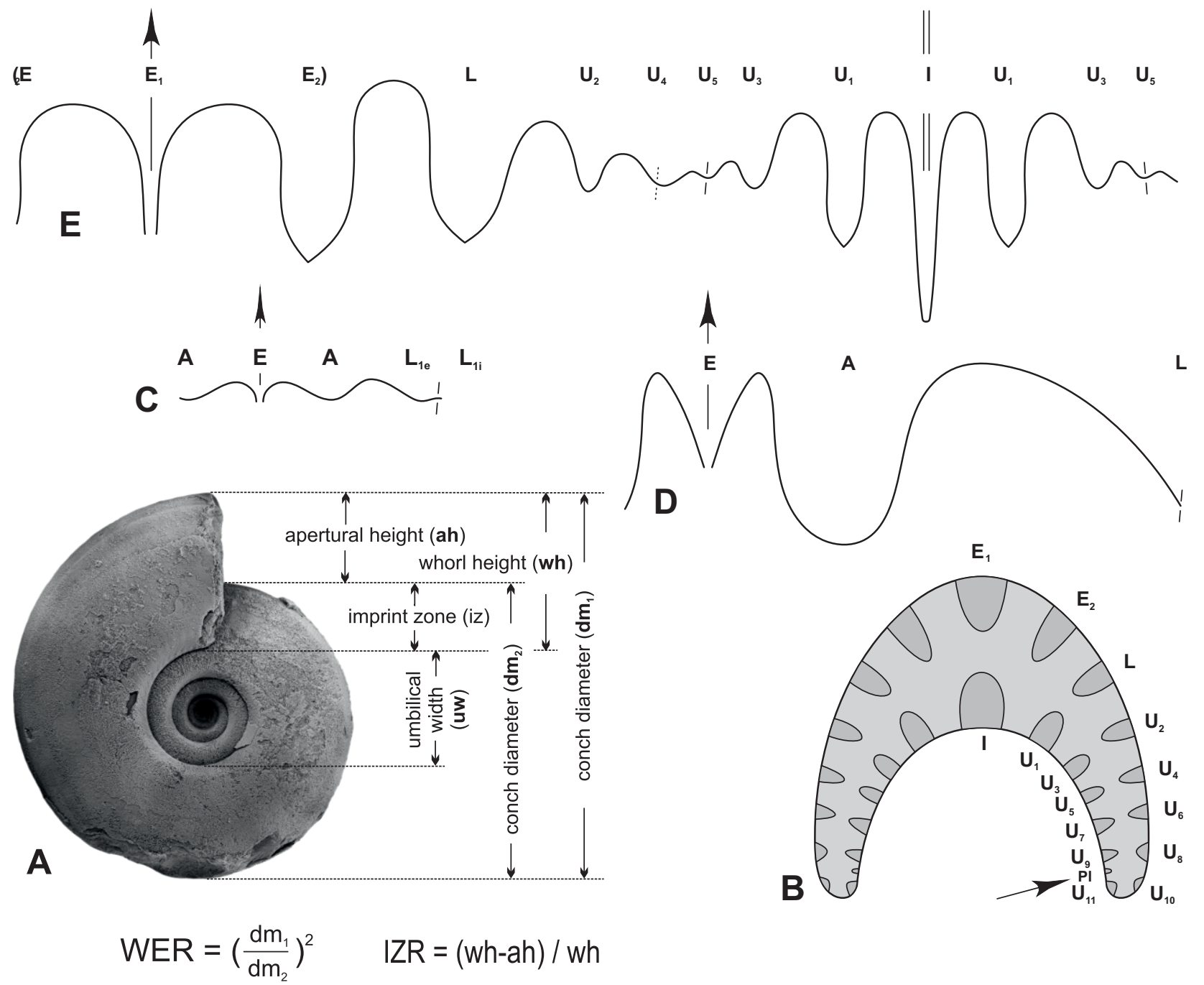

Figure 4. Overview of used conch parameters and abbreviations of suture elements; A. Conch parameters, their abbreviations and calculations. WER $=$ whorl expansion rate; IZR = imprint zone rate. B. Septal face and suture formula in Synpharciceras. C. Outer suture and its formula in Lobotornoceras. D. Outer suture and its formula in Epitornoceras. E. Suture and its formula in Extropharciceras. $\mathrm{E}=$ external lobe; $\mathrm{A}=$ adventitious lobe; $\mathrm{L}=$ lateral lobe; $\mathrm{U}=$ umbilical lobe; $\mathrm{I}=$ internal lobe; $\mathrm{Pl}=$ plurilobation. 


\section{Systematic Palaeontology}

Order Agoniatitida Ruzhencev, 1957

Suborder Gephuroceratina Ruzhencev, 1957

Superfamily Gephurocerataceae Frech, 1897

Family Acanthoclymeniidae Schindewolf, 1955

\section{Subfamily Ponticeratinae Korn \& Klug, 2002}

Diagnosis. Earliest whorls depressed, intermediate to mature conchs strongly compressed, subinvolute to evolute, with rounded, tabulate, or oxyconic venter, and often with ventrolateral furrows; growth lines biconvex, sometimes bundled ribs. Sutures with deep I lobe, shallow, internal $\mathrm{U}$ lobe, subumbilical, rounded or pointed L lobe, dominant, rounded, asymmetric or symmetric lateral saddle, incipient or deep $\mathrm{E}_{2}$ lobe, and narrow, deep or ontogenetically shortened $E_{1}$ lobe; suture formula: $\left(E_{2} E_{1} E_{2}\right) L$ : UI.

\section{Pseudoprobeloceras Bensaïd, 1974}

Type species. Pseudoprobeloceras nebechense Bensaïd, 1974, a subjective junior synonym of Ps. pernai.

Diagnosis: Small to large-sized, earliest whorls depressed, intermediate to mature conchs strongly compressed, subinvolute to evolute, with rounded venter and weak ventrolateral furrows; growth lines concavo-convex, bundled to ribs in early stages. Sutures with deep I lobe, shallow, internal $U$ lobe, rounded $L$ lobe, dominant, rounded, slightly asymmetric flank saddle, incipient $E_{2}$ lobe, and narrow, deep $E_{1}$ lobe; suture formula: $\left(\mathrm{E}_{2} \mathrm{E}_{1} \mathrm{E}_{2}\right) \mathrm{L}$ : UI.

\section{Pseudoprobeloceras pernai (Wedekind, 1918)}

Figures 5, 6

Lectotype. Original specimen figured by Wedekind (1918, pl. 21, fig. 1), designated in House \& Ziegler (1977), Marburg University collection. Type locality and horizon. Prinzkessel Ore Mine near Oberscheld, Dill Syncline, southern Rhenish Massive; late Givetian.

Material. More than 250 specimens up to $38 \mathrm{~mm}$ conch diameter, including MB.C.22134.1-MB.C.22134.26.

Diagnosis (emend.). Small-sized, conch ontogeny triphasic; first two whorls (up to $2 \mathrm{~mm} \mathrm{dm}$ ) moderately depressed, with increasing umbilical width ratio (uw/dm up to 0.45 ) and decreasing, low WER (down to 1.75); subsequent whorls with nearly constant conch parameters and identical ww/dm and uw/dm values; towards maturity with gradual, slight decrease of whorl (compressed from $10 \mathrm{~mm} \mathrm{dm}$ on, mature ww/wh $=\mathrm{ca}$. 0.75 ) and umbilical width rates (mature uw/dm $=0.30-0.35$ ) and strongly rising WER, reaching almost 2.50 ; umbilical wall shortly rounded, flanks slightly flattened, venter broadly rounded, bordered by rounded to subangular shoulders. Growth lines concavo-convex, with high ventrolateral salient lying in faint spiral furrows and with a deep ventral sinus. Ammonitella smooth, first two post-embryonic whorls with dense ribbing on inner flanks, subsequently coarser ribbed to nodose but ribs disappear gradually between 8 and $13 \mathrm{~mm} \mathrm{dm}$. Sutures with V-shaped, narrow I lobe, very shallow, wide internal U lobe, subsymmetric, deep and wide L lobe, slightly asymmetric, prominent EL saddle, very small, step-like $\mathrm{E}_{2}$ lobe, which becomes pointed in median stages, and very deep, narrow, diverging $\mathrm{E}_{1}$ lobe.

Table 1. Conch ontogeny (Figs 5A-H, L-N) of Pseudoprobeloceras pernai (Wedekind, 1918) from Hassi Nebech.

\begin{tabular}{llll}
\hline $\mathrm{dm}$ & conch shape & whorl cross-section shape & whorl expansion \\
\hline $2 \mathrm{~mm}$ & thinly to thickly discoidal; subevolute & moderately depressed; moderately embracing & low \\
& $($ ww/dm $=0.45-0.50 ;$ uw/dm $=0.40-0.45)$ & $($ ww/wh $=1.50-1.60 ;$ IZR $=0.20-0.25)$ & $($ WER $\sim 1.75)$ \\
$8 \mathrm{~mm}$ & thinly discoidal; subevolute & weakly depressed; moderately embracing & moderate to high \\
& $($ ww $/ \mathrm{dm} \sim 0.40 ;$ uw/dm $=0.35-0.45)$ & $($ ww/wh $\sim 1.10 ;$ IZR $=0.20-0.25)$ & $($ WER $=1.90-2.10)$ \\
$20 \mathrm{~mm}$ & extremely discoidal; subevolute & weakly compressed; moderately embracing & high \\
& $($ ww/dm $\sim 0.30 ;$ uw/dm $=0.30-0.35)$ & $($ ww/wh $=0.75-0.80 ;$ IZR $=0.20-0.25)$ & $($ WER $=2.00-2.20)$ \\
\hline
\end{tabular}

Table 2. Suture formula (Figs 5F, I-K), conch characteristics, and ornament of Pseudoprobeloceras pernai (Wedekind, 1918) from Hassi Nebech.

$\left(E_{2} E_{1} E_{2}\right) L: U I$

$E_{1}$ deep and straight; $E_{2}$ very small, shallow in juveniles, angular or steplike in adults; $E_{2} L$ saddle moderately wide and slightly asymmetric; $\mathrm{L}$ lobe deeply rounded; shallow $\mathrm{U}$ lobe; sometimes with asymmetric position of the I lobe (MB.C.22134.11)

venter rounded, nearly flat at about $25 \mathrm{~mm}$ dm; umbilicus moderately wide early stages until 8-13 mm dm with weak or strong (MB.C.22134.22) ribs; weak ventrolateral grooves in adults; no internal constrictions growth lines prorsiradiate, concavo-convex, with high ventrolateral projection 

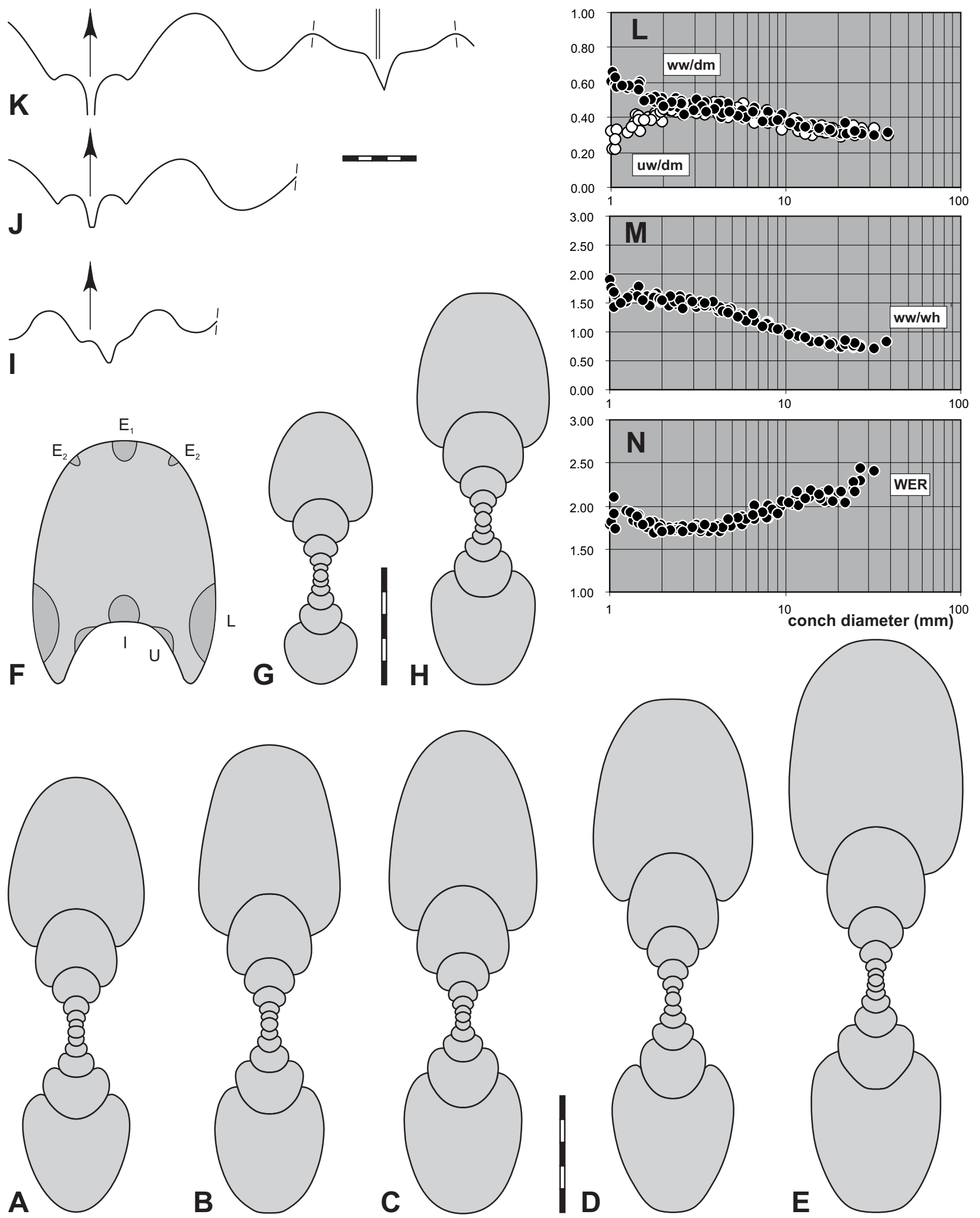

Figure 5. Pseudoprobeloceras pernai (Wedekind, 1918) from Hassi Nebech; A-H. Cross-sections, all $\times 4$; A. MB.C.22134.1; B. MB.C.22134.2; C. MB.C.22134.3; D. MB.C.22134.4; E. MB.C.22134.5; F. Septal face of MB.C.22134.6, ×4; G. MB.C.22134.7; H. MB.C.22134.8; I-K. Sutures, all ×2.5; I. MB.C.22134.9 at $6 \mathrm{~mm}$ wh; J. MB.C.22134.10 at $9 \mathrm{~mm}$ wh; K. MB.C.22134.11 at $11 \mathrm{~mm}$ wh; L-N. Ontogenetic development of ww $/ \mathrm{dm}, \mathrm{uw} / \mathrm{dm}$, ww/wh, and WER. Scale bar, 1 unit (black or white) $=1 \mathrm{~mm}$, also in the following Figures. 


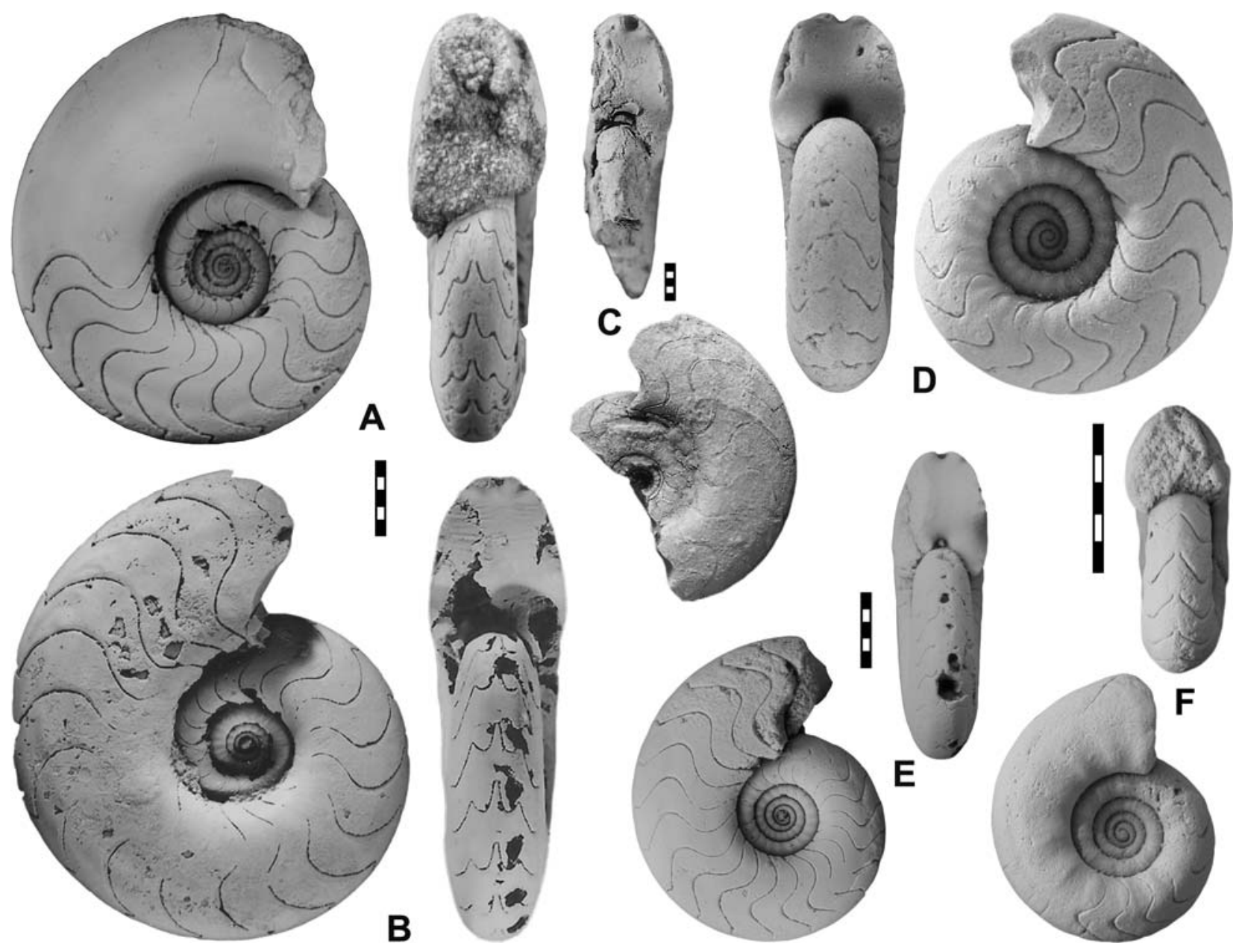

Figure 6. Pseudoprobeloceras pernai (Wedekind, 1918) from Hassi Nebech (except C); A. MB.C.22134.10, $\times 2$; B. MB.C.22134.12, $\times 2$; C. Lectotype of Manticoceras pontiforme Termier \& Termier (pl. 149, figs 3-5), $\times 1$; D. MB.C.22134.13 with "Mortońs Syndrome", ×4; E. MB.C.22134.14, ×2; F. MB.C.22134.15, $\times 4$.

\section{Pseudoprobeloceras praecox n. sp.}

Figures 7, 8

Derivation of name. From the Latin word for premature; because of the early ontogenetic expression of rapidly expanding, compressed whorls. Holotype. MB.C.22135.1, illustrated in Figures 7E and 8A.

Type locality and horizon. Hassi Nebech, Section 2, SE Tafilalt, main collecting level, probably Taouzites taouzensis Zone (late Givetian). Material. More than 50 specimens up to $20 \mathrm{~mm}$ conch diameter including MB.C.22135.1-MB.C.22135.16.

Diagnosis. Small-sized, post-embryonic conch ontogeny slightly biphasic; first three whorls (up to ca. $3.5 \mathrm{~mm} \mathrm{dm}$ ) low, depressed, with moderate WER $<2.00$ and rising umbilical width rate (uw/dm up to almost 0.40 ); subsequently with increasingly less depressed to compressed (from $9 \mathrm{~mm} \mathrm{dm}$ on) and rapidly expanding whorls (WER gradually high to very high, $>2.50$ at maturity) and increasingly subevolute to subinvolute (uw/dm $0.20-$ 0.25 at maturity); early whorls well-rounded, mature whorls flattened but with rounded ventral and umbilical shoulders. Early stages up to 7-9 mm $\mathrm{dm}$ ribbed, mature whorls smooth; growth lines concavo-convex, with projecting ventrolateral salient lying in shallow spiral furrows. Mature sutures with deep $L$ lobe, wide and high ventrolateral saddle, both slightly asymmetric, small, pointed $\mathrm{E}_{2}$ lobe and deep, diverging $\mathrm{E}_{1}$ lobe.

Table 3. Conch ontogeny (Figs 7A-C, F-H) of Pseudoprobeloceras praecox $\mathrm{n}$. sp.

\begin{tabular}{llll}
\hline $\mathrm{dm}$ & conch shape & whorl cross-section shape & whorl expansion \\
\hline $2 \mathrm{~mm}$ & thickly discoidal; subevolute & moderately depressed; moderately embracing & moderate \\
& $($ ww/dm $\sim 0.55 ;$ uw/dm $\sim 0.35)$ & (ww/wh $\sim 1.50 ;$ IZR $=0.25-0.30)$ & $($ WER $\sim 1.90)$ \\
$8 \mathrm{~mm}$ & thinly discoidal; subinvolute & weakly depressed; moderately embracing & high \\
& $($ ww/dm $\sim 0.45 ;$ uw $/ \mathrm{dm} \sim 0.30)$ & $($ ww/wh $=1.00-1.10 ;$ IZR $\sim 0.25)$ & $($ WER $=2.10-2.20)$ \\
$20 \mathrm{~mm}$ & extremely discoidal; subinvolute & weakly compressed; moderately embracing & very high \\
& $($ ww $/ \mathrm{dm}=0.30-0.35 ;$ uw $/ \mathrm{dm} \sim 0.25)$ & $($ ww/wh $=0.70-0.80 ;$ IZR $\sim 0.20)$ & $($ WER $=2.30-2.50)$ \\
\hline
\end{tabular}


Table 4. Suture formula (Figs 7D, E), conch characteristics, and ornament of Pseudoprobeloceras praecox n. $\mathrm{sp}$.

\section{$\left(E_{2} E_{1} E_{2}\right) L$ : (probably U) I}

$E_{1}$ short; $E_{2}$ very small and steplike; $L$ lobe deeply rounded, $E_{2} L$ saddle moderately wide; $L$ lobe deeply rounded; both slightly asymmetric venter narrowly rounded, nearly flat at about $20 \mathrm{~mm}$ dm; umbilicus moderately narrow juvenile ribs; weak furrows in adults; no internal mould constrictions growth lines concavo-convex, with high ventrolateral salient

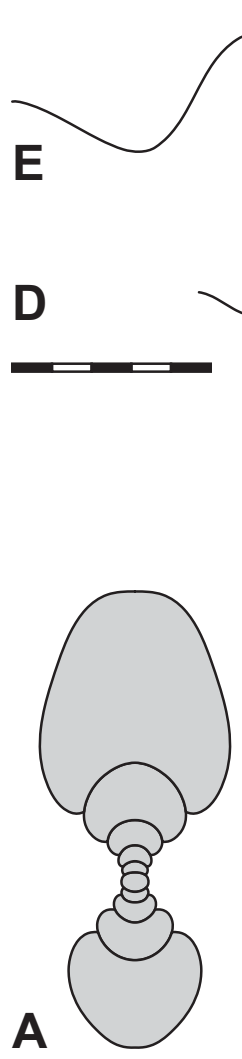

B
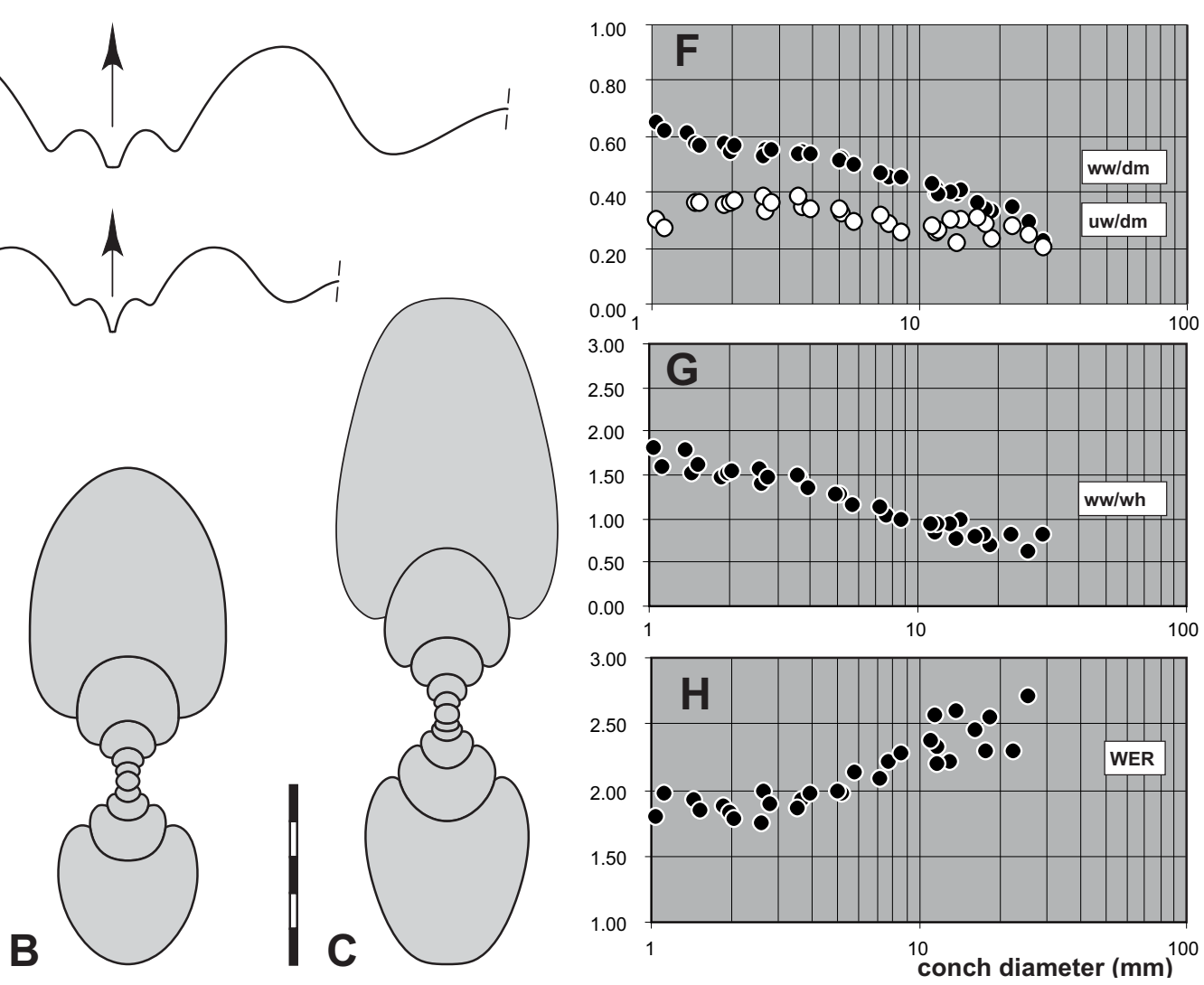

Figure 7. Pseudoprobeloceras praecox n. sp. from Hassi Nebech; A. Cross-section of paratype MB.C.22135.2, $\times 4$; B. Cross-section of paratype MB.C.22135.3, $\times 4$; C. Cross-section of paratype MB.C.22135.4, $\times 4$; D. Suture of paratype MB.C.22135.5 at $4.7 \mathrm{~mm}$ wh, $\times 4$; E. Suture of holotype MB.C.22135.1 at $9.4 \mathrm{~mm}$ wh, $\times 4 ; \mathbf{F}-\mathbf{H}$. Ontogenetic development of ww/dm, uw/dm, ww/ wh, and WER.
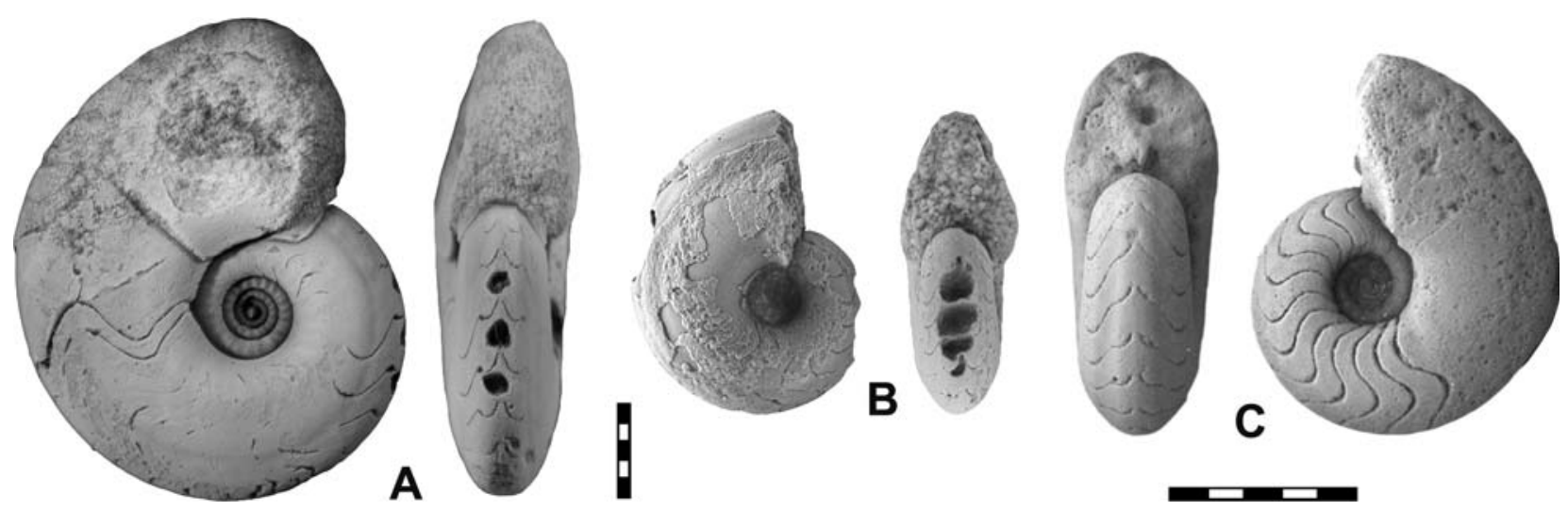

Figure 8. Pseudoprobeloceras praecox n. sp. from Hassi Nebech; A. Holotype MB.C.22135.1, ×2; B. Paratype MB.C.22135.6, $\times 2$; C. Paratype MB.C. $22135.7, \times 4$. 


\section{Subfamily Acanthoclymeniinae Schindewolf, 1955}

Diagnosis. Very small to medium-sized, earliest whorls depressed, median to mature conch strongly compressed, subinvolute to evolute, venter gently rounded, tabulate or bicarinate, often with ventrolateral furrows; growth lines concavo-convex to biconvex. Sutures with deep I lobe, absent, shallow or moderately deep, rounded or pointed internal U lobe, subumbilical, rounded or V-shaped L lobe, dominant, (sub)triangular ventrolateral saddle, incipient or deep $E_{2}$ lobe, and narrow, deep or ontogenetically shortened, $E_{1}$ lobe; suture formula: $\left(E_{2} E_{1} E_{2}\right) L: I$.

\section{Scaturites n. gen.}

Derivation of name. After the Latin scaturire $=$ to spring and the Greek ites $=$ stone; due to its basal position within the Acanthoclymeniinae. Type species. Scaturites minutus $\mathrm{n}$. sp.

Diagnosis. Very small sized, smooth, earliest whorls depressed, intermediate to mature conch compressed, subevolute, with rounded venter and faint ventrolateral furrows; growth lines concavo-convex with high ventrolateral projection. Sutures with deep I lobe, widely rounded L lobe, strongly asymmetric, relative narrow and subtriangular ventrolateral saddle, incipient $\mathrm{E}_{2}$ lobe, and narrow, deep $\mathrm{E}_{1}$ lobe; suture formula: $\left(\mathrm{E}_{2} \mathrm{E}_{1} \mathrm{E}_{2}\right) \mathrm{L}: \mathrm{I}$.

Included species. Sc. minutus n. sp., ?Pseudoprobeloceras cf. nebechense in Göddertz (1989)

Geographic distribution and stratigraphic range. North Africa (Tafilalt, ? southern Algeria); late Givetian.

\section{Scaturites minutus n. sp.}

Figures 9,10

Derivation of name. Because of the small size.

Holotype. MB.C.22136.1, illustrated in Figures 9G and 10A.

Type locality and horizon. Hassi Nebech, Section 2, SE Tafilalt, main collecting level, probably Taouzites taouzensis Zone (late Givetian). Material. More than 200 specimens up to $15 \mathrm{~mm}$ conch diameter including MB.C.22136.1-MB.C.22136.13.

Diagnosis. Very small-sized, first two rounded whorls up to ca. $2.3 \mathrm{~mm} \mathrm{dm}$ weakly depressed, with increasing umbilical width (uw/dm up to ca. 0.42 ) and decreasing whorl expansion rate (down to $<2.00$ ); subsequently increasingly compressed (from ca. $4 \mathrm{~mm} \mathrm{dm}$ on), subevolute (mature $\mathrm{uw} / \mathrm{dm}=$ ca. 0.32 ), and with sharply rising WER (up to 2.50); whorls laterally flattened; WER decreases on the last whorl $(>10 \mathrm{~mm} \mathrm{dm})$ while
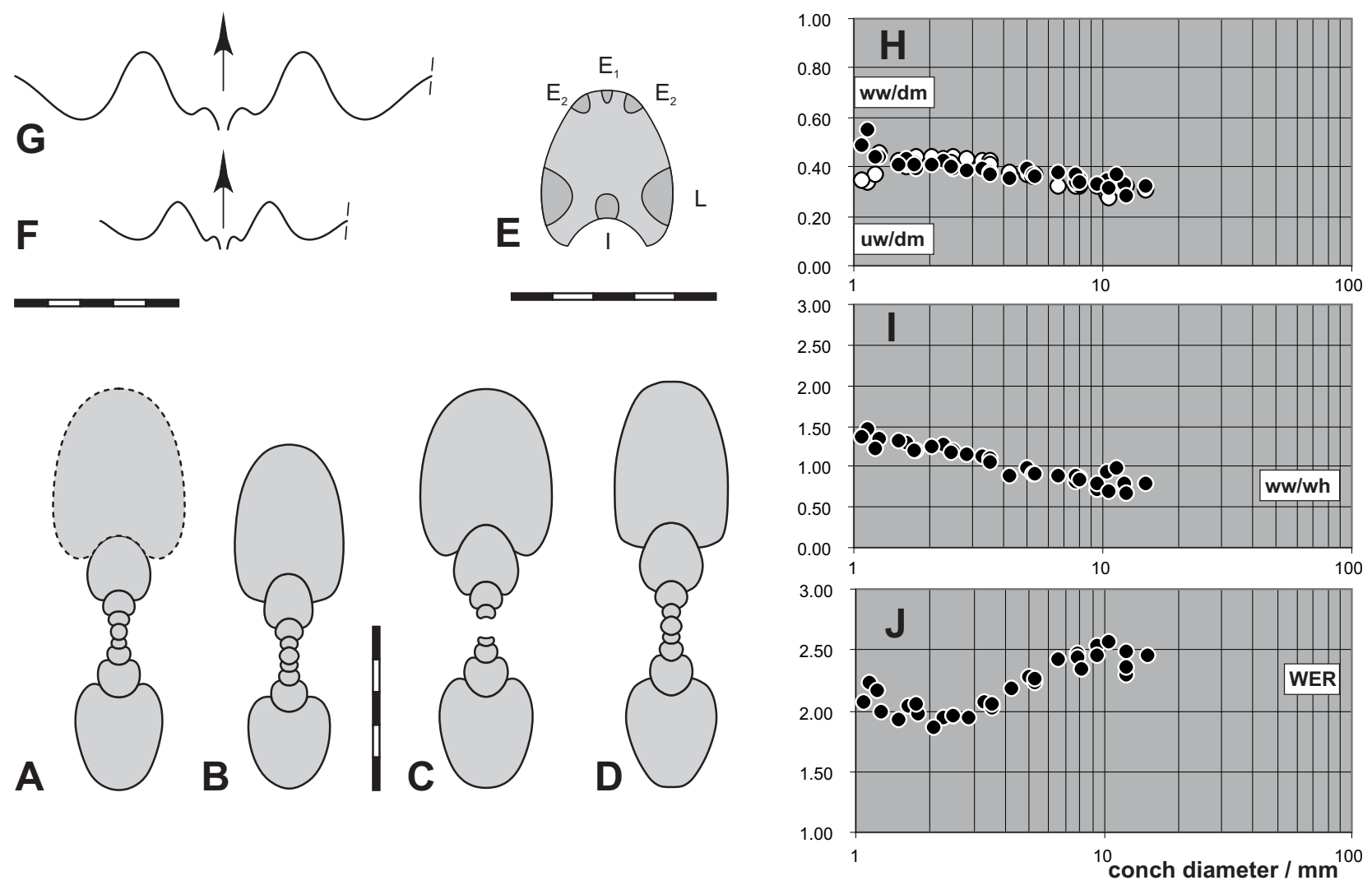

Figure 9. Scaturites minutus n. gen. n. sp. from Hassi Nebech; A-D. Cross-sections, all $\times$ 4; A. Paratype MB.C.22136.2; B. Paratype MB.C.22136.3; C. Paratype MB.C.22136.4; D. Paratype MB.C.22136.5; E. Septal face of paratype MB.C.22136.6, ×5; F. Suture of paratype MB.C.22136.7 at $2.9 \mathrm{~mm}$ wh, $\times 4$; G. Suture of holotype MB.C.22136.1 at $4.4 \mathrm{~mm}$ wh; $\times 4$, H-J. Ontogenetic development of ww/dm, uw/dm, ww/wh, and WER. 

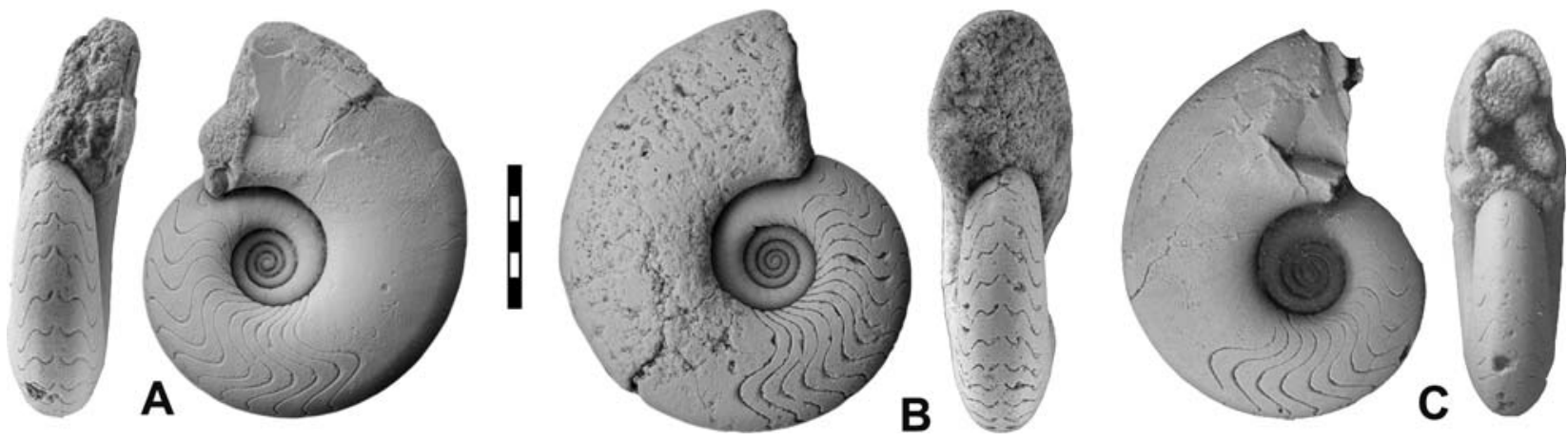

Figure 10. Scaturites minutus n. gen. n. sp. from Hassi Nebech; A. Holotype MB.C.22136.1; B. Paratype MB.C.22136.8; C. Paratype MB.C.22136.9; all $\times 3$.

Table 5. Conch ontogeny (Figs $9 \mathrm{~A}-\mathrm{E}, \mathrm{H}-\mathrm{J}$ ) of Scaturites minutus n. gen. n. sp.

\begin{tabular}{|c|c|c|c|}
\hline$d m$ & conch shape & whorl cross-section shape & whorl expansion \\
\hline $2 \mathrm{~mm}$ & $\begin{array}{l}\text { thinly discoidal; subevolute } \\
\text { (ww/dm } \sim 0.40 ; \text { uw/dm }=0.40-0.45 \text { ) }\end{array}$ & $\begin{array}{l}\text { weakly depressed; weakly to mod. embracing } \\
\text { (ww/wh }=1.20-1.30 ; \text { IZR }=0.10-0.20 \text { ) }\end{array}$ & $\begin{array}{l}\text { moderate } \\
(\mathrm{WER}=1.90-2.00)\end{array}$ \\
\hline $8 \mathrm{~mm}$ & $\begin{array}{l}\text { thinly discoidal; subevolute } \\
\text { (ww/dm } \sim 0.35 ; \text { uw/dm } \sim 0.33 \text { ) }\end{array}$ & $\begin{array}{l}\text { weakly compressed; weakly to mod. embracing } \\
\text { (ww/wh }=0.80-0.85 ; \text { IZR } \sim 0.10-0.20 \text { ) }\end{array}$ & $\begin{array}{l}\text { high } \\
(\text { WER }=2.10-2.20)\end{array}$ \\
\hline $12 \mathrm{~mm}$ & $\begin{array}{l}\text { extremely discoidal; subevolute } \\
(\mathrm{ww} / \mathrm{dm}=0.30 .0 .35 ; \mathrm{uw} / \mathrm{dm} \sim 0.32)\end{array}$ & $\begin{array}{l}\text { weakly compressed; weakly to mod. embracing } \\
\text { (ww/wh }=0.70-0.95 ; \text { IZR } \sim 0.10-0.20 \text { ) }\end{array}$ & $\begin{array}{l}\text { very high } \\
(\text { WER }=2.35-2.50)\end{array}$ \\
\hline
\end{tabular}

Table 6. Suture formula (Figs 9E-G), conch characteristics, and ornament of Scaturites minutus n. gen. n. sp.

$\left(E_{2} E_{1} E_{2}\right) L: I$

$E_{1}$ short; $E_{2}$ very small, rounded; $E_{2} L$ saddle subtriangular; $L$ lobe deep and widely rounded

venter narrowly rounded or nearly flat; umbilicus moderately wide

no ribs in juvenils or adults; sometimes very shallow furrows; no internal constrictions

growth lines prorsiradiate, concavo-convex (MB.C.22136.12, MB.C.22136.13)

the other conch parameter ratios stagnate. Smooth throughout ontogeny apart from rare traces of ventrolateral furrows. Sutures with small, deep $\mathrm{E}_{1}$ lobe, very small, step-like $\mathrm{E}_{2}$ lobes, narrow, subtriangular ventrolateral saddle, deep, wide, slightly asymmetric L lobe, and deep, narrow I lobe.

Family Taouzitidae Korn, 2001

\section{Darkaoceras Bockwinkel, Becker \& Ebbighausen, 2009}

Type species. Timanites meridionalis Petter, 1959.

For a detailed discussion of the genus see Bockwinkel et al. (2009).

\section{Darkaoceras velox n. sp.}

Figures 11, 12

Derivation of name. From the Latin velox = fast; because of the stream-lined, compressed conch, which allowed relatively fast horizontal swimming (Klug \& Korn 2004).

Holotype. MB.C.22105.1, illustrated in Figures 11I and 12B, one of the largest available specimens, showing the characteristic conch shape and sutures.

Type locality and horizon. Hassi Nebech, Section 2, SE Tafilalt, main collecting level, probably Taouzites taouzensis Zone (late Givetian).

Material. More than 50 specimens up to $30 \mathrm{~mm}$ conch diameter, including MB.C.22105.1-MB.C.22105.22.

Diagnosis. First three whorls (until ca. $5.5 \mathrm{~mm} \mathrm{dm}$ ) moderately depressed (, ww/wh falling from 2.00 to 1.50 ), with moderate WER (1.752.00 ), subevolute (uw/dm $=0.35-0.40$ ), and with weak to pronounced subumbilical ribbing and rounded venter; subsequent intermediate to 


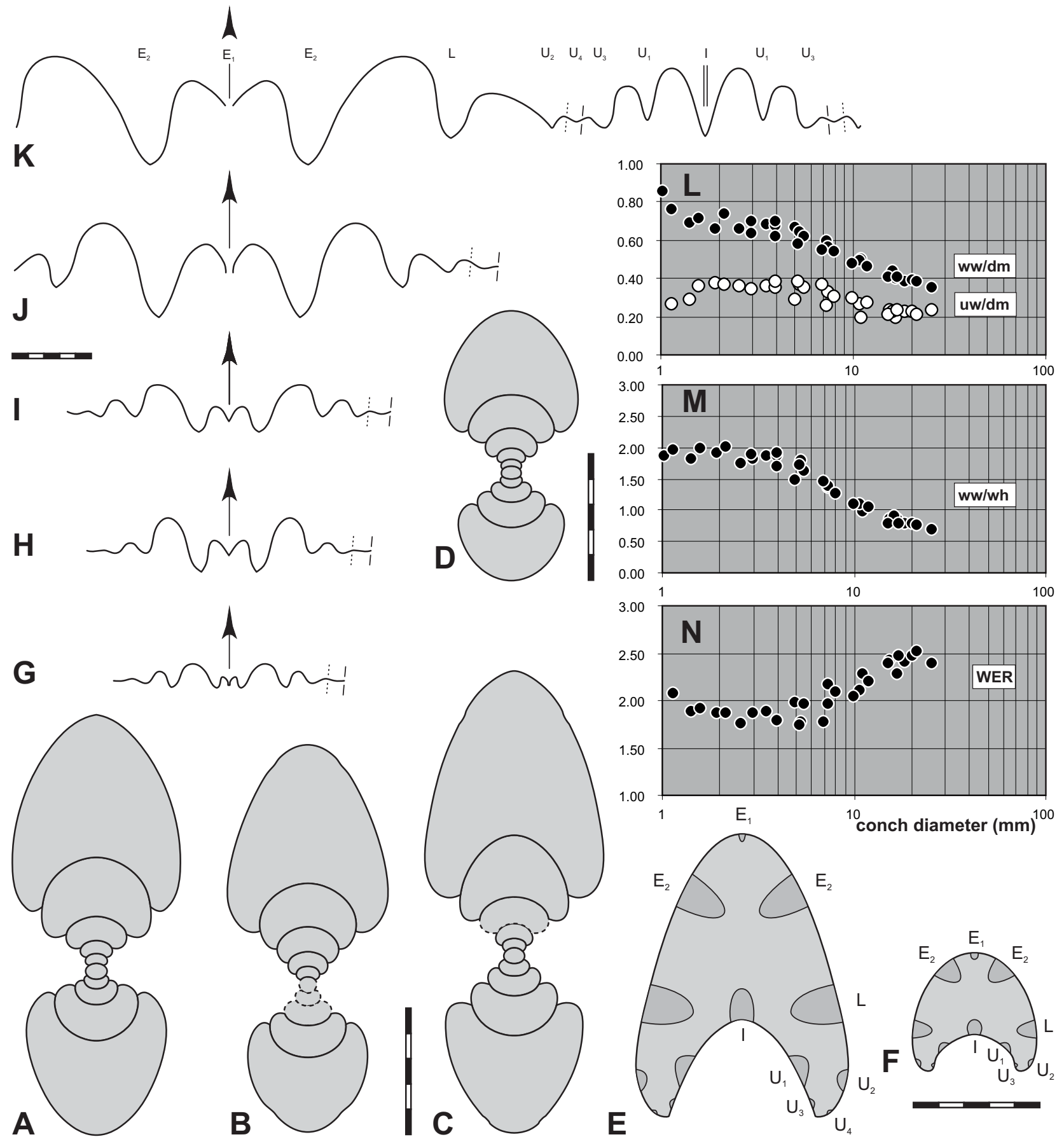

Figure 11. Darkaoceras velox n. sp. from Hassi Nebech; A-D. Cross-sections, all $\times 4$; A. Paratype MB.C.22105.2; B. Paratype MB.C.22105.3; C. Paratype MB.C.22105.4; D. Paratype MB.C.22105.5; E. Septal face of paratype MB.C.22105.6, ×4; F. Septal face of paratype MB.C.22105.7, $\times 4$; G-K. Sutures, all $\times 2.5$; G. Paratype MB.C.22105.8 at $6.1 \mathrm{~mm}$ wh; H. Paratype MB.C.22105.9 at $6.9 \mathrm{~mm}$ wh; I. Holotype MB.C.22105.1 at $7.3 \mathrm{~mm}$ wh; J. Paratype MB.C.22105.10 at $13.5 \mathrm{~mm}$ wh; K. Paratype MB.C.22105.11 at ca. $21 \mathrm{~mm}$ wh; L-N. Ontogenetic development of ww/dm, uw/dm, ww/wh, and WER.

mature stages increasingly less depressed to compressed (from ca. $10 \mathrm{~mm} \mathrm{dm}$ on), mature whorls $(>20 \mathrm{~mm} \mathrm{dm}$ ) with ww/dm $<0.40$, with gradual sharpening of the venter, decreasing umbilical width rates (mature uw/dm down to ca. 0.20 ) and strongly rising WER (2.50 at maturity), and disappearance of ribs. Growth lines undulose and strongly biconvex, with deep ocular sinus and high ventrolateral projection lying in a broad spiral furrow or double furrow. Mature sutures with shallow, divergent $\mathrm{E}_{1}$ lobe, high median saddle, deep, pointed, roughly V-shaped $\mathrm{E}_{2}$ lobe, high, broadly arched ventrolateral saddle, narrow, pointed $\mathrm{L}$ lobe, less deep than the $\mathrm{E}_{2}$ lobe, relatively wide, low, asymmetric $\mathrm{LU}_{2}$ saddle, small outer $\mathrm{U}_{2}$ and $\mathrm{U}_{4}$ lobes, small internal $\mathrm{U}_{3}$ lobe, narrow, moderately deep, pointed $\mathrm{U}_{1}$ lobe, and narrow, deep, pointed I lobe. 

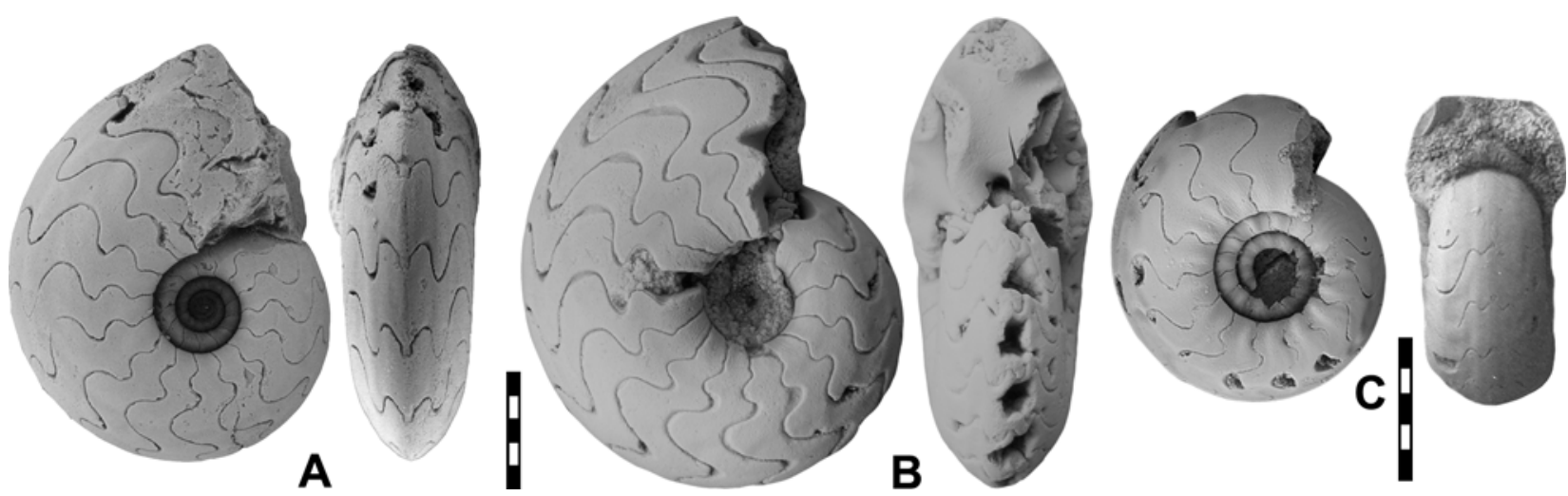

Figure 12. Darkaoceras velox n. sp. from Hassi Nebech; A. Paratype MB.C.22105.12, $\times 2.5$; B. Holotype MB.C.22105.1, $\times 2.5$; C. Paratype MB.C.22105.13, $\times 3$.

Table 7. Conch ontogeny (Figs $11 \mathrm{~A}-\mathrm{F}, \mathrm{L}-\mathrm{N}$ ) of Darkaoceras velox $\mathrm{n}$. sp.

\begin{tabular}{|c|c|c|c|}
\hline $\mathrm{dm}$ & conch shape & whorl cross-section shape & whorl expansion \\
\hline $2 \mathrm{~mm}$ & $\begin{array}{l}\text { thinly to thickly pachyconic; subevolute } \\
\text { (ww/dm }=0.65-0.75 ; \text { uw } / \mathrm{dm}=0.35-0.40 \text { ) }\end{array}$ & $\begin{array}{l}\text { moderately depressed ; moderately embracing } \\
(w w / w h \sim 2.00 ; \operatorname{IZR}=0.25-0.30)\end{array}$ & $\begin{array}{l}\text { moderate } \\
(\text { WER } \sim 1.85)\end{array}$ \\
\hline $5 \mathrm{~mm}$ & $\begin{array}{l}\text { thickly discoidal to thinly pachyconic; } \\
\text { subevolute to subinvolute } \\
\text { (ww/dm }=0.55-0.65 ; \text { uw/dm }=0.25-0.40 \text { ) }\end{array}$ & $\begin{array}{l}\text { moderately depressed; strongly embracing } \\
(\mathrm{ww} / \mathrm{wh}=1.50-1.80 ; \mathrm{IZR}=0.30-0.35)\end{array}$ & $\begin{array}{l}\text { moderate } \\
(\mathrm{WER}=1.75-2.00)\end{array}$ \\
\hline $15 \mathrm{~mm}$ & $\begin{array}{l}\text { thinly discoidal; subinvolute } \\
(\mathrm{ww} / \mathrm{dm}=0.40-0.45 ; \mathrm{uw} / \mathrm{dm}=0.20-0.25)\end{array}$ & $\begin{array}{l}\text { weakly compressed; moderately embracing } \\
(\mathrm{ww} / \mathrm{wh}=0.80-0.90 ; \mathrm{IZR}=0.25-0.30\end{array}$ & $\begin{array}{l}\text { very high } \\
(\text { WER }=2.30-2.40)\end{array}$ \\
\hline $25 \mathrm{~mm}$ & $\begin{array}{l}\text { thinly discoidal; subinvolute } \\
(\text { ww/dm } \sim 0.35 ; \text { uw/dm } \sim 0.25)\end{array}$ & $\begin{array}{l}\text { weakly compressed; moderately embracing } \\
\text { (ww/wh } \sim 0.70 ; \text { IZR } \sim 0.30 \text { ) }\end{array}$ & $\begin{array}{l}\text { very high } \\
(\text { WER } \sim 2.40)\end{array}$ \\
\hline
\end{tabular}

Table 8. Suture formula (Figs 11E-K), conch characteristics, and ornament of Darkaoceras velox n. sp.

$\left(E_{2} E_{1} E_{2}\right) L U_{2}: U_{3} U_{1} l$ at $9 \mathrm{~mm} d m$ (MB.C.22105.7)

\section{$\left(E_{2} E_{1} E_{2}\right) L U_{2} U_{4}: U_{3} U_{1} l$ at $13 \mathrm{~mm}$ dm (MB.C.22105.20)}

$\left(\mathrm{E}_{2} \mathrm{E}_{1} \mathrm{E}_{2}\right) \mathrm{LU}_{2} \mathrm{U}_{4}: \mathrm{U}_{3} \mathrm{U}_{1} \mathrm{l}$ a t 20 and $40 \mathrm{~mm}$ dm (MB.C.22105.15, MB.C.22105.11)

$\mathrm{E}_{1}$ lobe broad, $\mathrm{V}$ - or funnel-shaped, only $\sim 30 \%$ depth of $\mathrm{E}_{2}$ lobe at maturity; $\mathrm{E}_{2}$ lobe deep and large; ventrolateral saddle high and broadly rounded, asymmetric; $L$ lobe short; outer $U$ lobes small

venter suboxyconic at ca. $13 \mathrm{~mm} \mathrm{dm}$, oxyconic at $15-30 \mathrm{~mm} \mathrm{dm}$

juvenile stages weak or distinctive ribs; ventrolateral (double) furrows; no internal constrictions

growth lines rectiradiate, strongly biconvex, with high ventrolateral salient

\section{Taouzites Korn, 2001}

Type species. Pharciceras taouzensis Termier \& Termier, 1950.

Diagnosis (emend.). Large-sized, early whorls weakly depressed and subevolute, ribbed and with rounded venter; intermediate to mature whorls rapidly expanding, thinly to extremely discoidal, oxyconic, without ribs and with strongly decreasing umbilical width ratios. Ornament strongly biconvex with high ventrolateral salient lying in spiral furrows, which weaken with growth. Sutures with short, strongly divergent $\mathrm{E}_{1}$ lobe, high median saddle, moderately deep to deep, pointed $\mathrm{E}_{2}$ lobe, dominant, wide $\mathrm{E}_{2} \mathrm{~L}$ saddle, deep, pointed $\mathrm{L}$ lobe, and ontogenetically with up to five outer and three inner $U$ lobes, which do not develop in a strictly alternating order. Suture formula: $\left(E_{2} E_{1} E_{2}\right) L_{2} U_{4} U_{5} U_{7}: U_{6} U_{3} U_{1} I$ to $\left(\mathrm{E}_{2} \mathrm{E}_{1} \mathrm{E}_{2}\right) \mathrm{LU}_{2} \mathrm{U}_{4} \mathrm{U}_{5} \mathrm{U}_{7} \mathrm{U}_{8}: \mathrm{U}_{6} \mathrm{U}_{3} \mathrm{U}_{1} \mathrm{I}$.

\section{Taouzites taouzensis (Termier \& Termier, 1950)}

Figures 13, 14

Lectotype. Termier \& Termier (1950) and Petter (1959) based their description of "Pharciceras" taouzense on the Clariond collection, which contains the syntype material. Montesinos \& Henn (1986) designated Petter's original of pl. 9, figs 7, 7a, as lectoptype, which is accepted here although it was not figured in the valid original description.

Type locality and horizon. Oued Kseir = Hassi Nebech area, SE Tafilalt, main collecting level, Taouzites taouzensis Zone (late Givetian). 


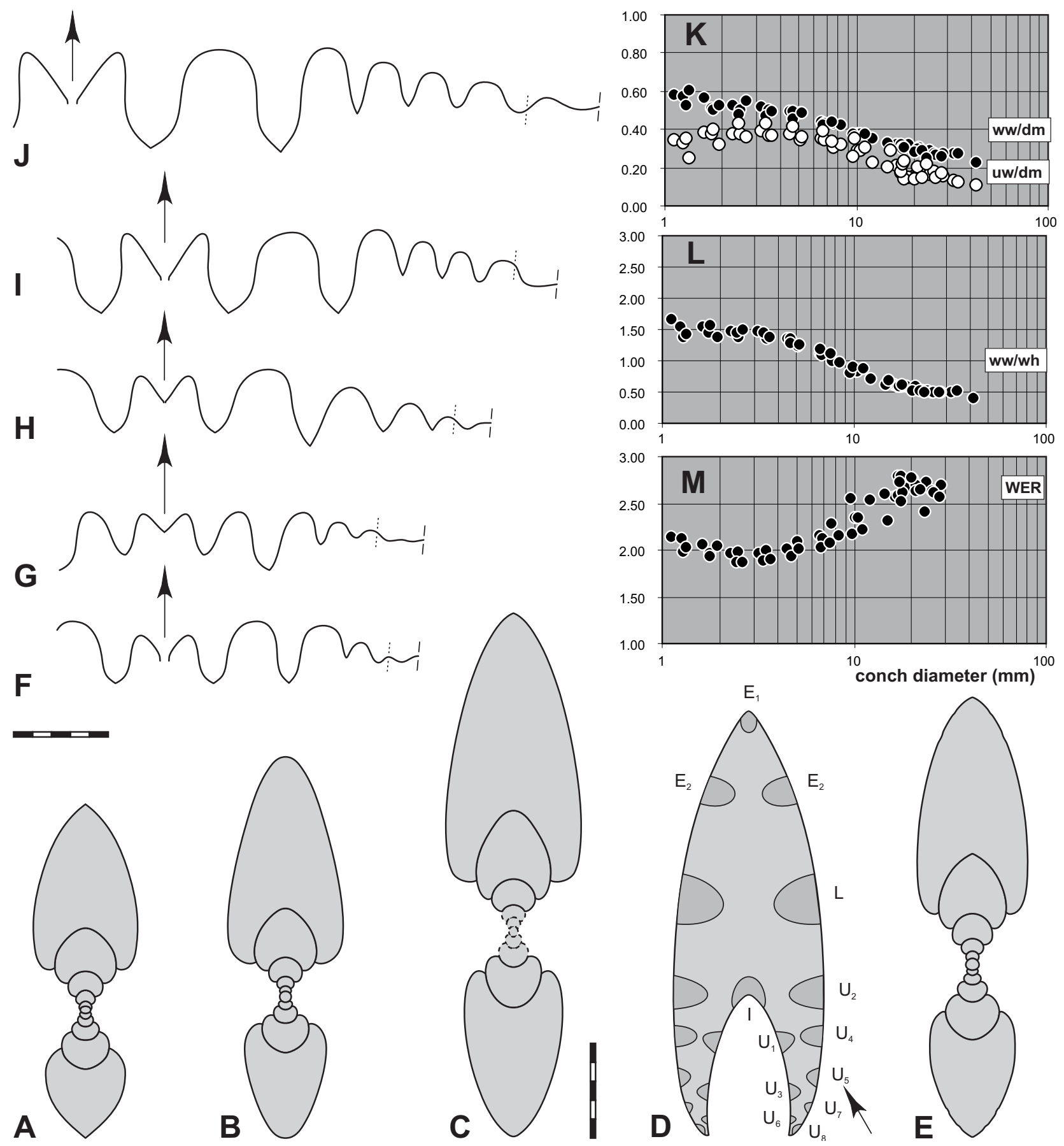

Figure 13. Taouzites taouzensis (Termier \& Termier, 1950), all topotypes from Hassi Nebech (exept D); A. Cross-section of MB.C.22106.1, $\times 3$; B. Cross-section of MB.C.22106.2, $\times 3$; C. Cross-section of MB.C.22106.3, $\times 3$; D. Septal face of MB.C.22138 from Ouidane Chebbi, $\times 3$; E. Septal face of MB.C.22106.4, $\times 3$; F-J. Sutures, all $\times 3$; F. MB.C.22106.4 at $10.7 \mathrm{~mm}$ wh; G. MB.C.22106.5 at $11.2 \mathrm{~mm}$ wh; H. MB.C.22106.6 at $14.5 \mathrm{~mm}$ wh; I. MB.C.22106.7 at $16 \mathrm{~mm}$ wh; J. MB.C.22106.7 at $20 \mathrm{~mm}$ wh; K-M. Ontogenetic development of ww/dm, uw/dm, ww/wh, and WER.

Material. More than 300 specimens (Hassi Nebech topotypes) up to $42 \mathrm{~mm}$ conch diameter, including MB.C.22106.1-MB.C.22106.23; one specimen from Ouidane Chebbi (eastern Tafilalt) (MB.C.22138), showing a larger septal face (Fig. 13D).

Diagnosis. (emend.) First two whorls (up to ca. $3 \mathrm{~mm} \mathrm{dm}$ ) weakly depressed (ww/wh ca. 1.50) and increasingly subevolute (uw/dm up to 0.40 ) and with slightly decreasing WER $(<2.00)$; median stages up to ca. $20 \mathrm{~mm} \mathrm{dm}$ less depressed to increasingly compressed (from ca. $8 \mathrm{~mm} \mathrm{dm}$, $w w / \mathrm{dm}$ falling from 0.50 to 0.30 ), subinvolute (uw/dm down to 0.20 ) and with high to very high WER (up to 2.80); mature conchs strongly compressed, strongly oxyconic, with $\mathrm{uw} / \mathrm{dm}=\mathrm{ca}$. 0.15 . Growth ornament strongly biconvex, forming dense inner flank ribs in early whorls, and with very high, narrow ventrolateral salient lying in spiral double furrows. Mature sutures with divergent, V-shaped, moderately deep $E_{1}$ lobe, narrow median saddles, moderately deep to deep, bell-shaped $\mathrm{E}_{2}$ lobe, prominent, wide, slightly asymmetric $\mathrm{E}_{2} \mathrm{~L}$ saddle, deep, narrow and pointed $\mathrm{L}$ lobe, pointed small outer $\mathrm{U}_{2}$ and $\mathrm{U}_{4}$ lobes, and three further outer $\mathrm{U}$ lobes, but only three inner $\mathrm{U}$ lobes. Mature suture formula: $\left(\mathrm{E}_{2} \mathrm{E}_{1} \mathrm{E}_{2}\right) \mathrm{LU}_{2} \mathrm{U}_{4} \mathrm{U}_{5} \mathrm{U}_{7} \mathrm{U}_{8}: \mathrm{U}_{6} \mathrm{U}_{3} \mathrm{U}_{1} \mathrm{I}$. 

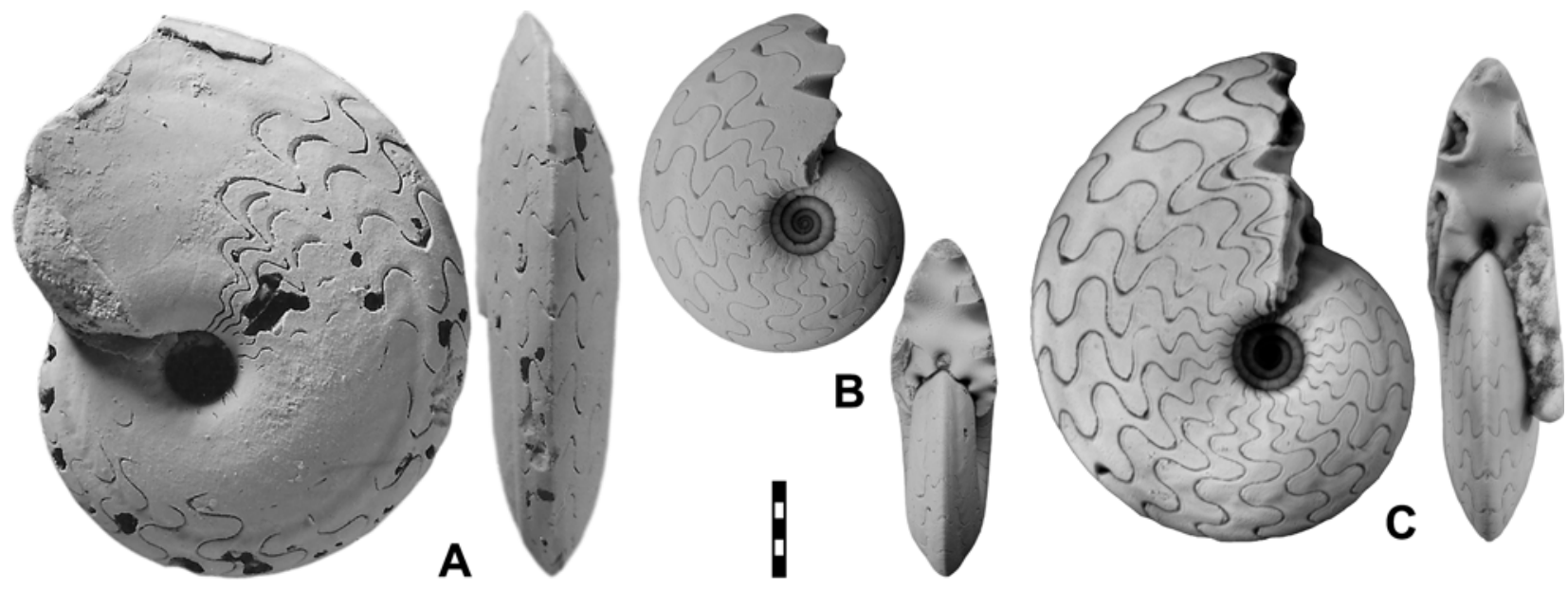

Figure 14. Taouzites taouzensis (Termier \& Termier, 1950) from Hassi Nebech; A. MB.C.22106.20; B. MB.C.22106.8; C. MB.C. 22106.9 ; all $\times 2$.

Table 9. Conch ontogeny (Figs 13A-C, E, K-M) of Taouzites taouzensis (Termier \& Termier, 1950) from the type locality.

\begin{tabular}{|c|c|c|c|}
\hline $\mathrm{dm}$ & conch shape & whorl cross-section shape & whorl expansion \\
\hline $2 \mathrm{~mm}$ & $\begin{array}{l}\text { thickly discoidal; subevolute } \\
(\text { ww } / \mathrm{dm}=0.50-0.55 ; \text { uw/dm }=0.30-0.40)\end{array}$ & $\begin{array}{l}\text { weakly depressed; moderately embracing } \\
(\mathrm{ww} / \mathrm{wh}=1.40-1.50 ; \text { IZR } \sim 0.20)\end{array}$ & $\begin{array}{l}\text { moderate } \\
\text { to high }(\text { WER } \sim 2.00)\end{array}$ \\
\hline $10 \mathrm{~mm}$ & $\begin{array}{l}\text { thinly discoidal; subevolute to subinvolute } \\
\text { (ww/dm }=0.35-0.40 ; u w / d m=0.25-0.35 \text { ) }\end{array}$ & $\begin{array}{l}\text { weakly compressed; moderately embracing } \\
(\mathrm{ww} / \mathrm{wh}=0.80-1.00 ; \mathrm{IZR} \sim 0.23 \text { ) }\end{array}$ & $\begin{array}{l}\text { very high } \\
\text { (WER }=2.30-2.55)\end{array}$ \\
\hline $20 \mathrm{~mm}$ & $\begin{array}{l}\text { extremely discoidal; subinvolute } \\
(\mathrm{ww} / \mathrm{dm} \sim 0.30 ; \mathrm{uw} / \mathrm{dm}=0.15-0.25)\end{array}$ & $\begin{array}{l}\text { weakly compressed; moderately embracing } \\
(\mathrm{w} w / \mathrm{wh}=0.50-0.60 ; \mathrm{IZR} \sim 0.28)\end{array}$ & $\begin{array}{l}\text { extremely high } \\
\text { (WER }=2.60-2.80)\end{array}$ \\
\hline
\end{tabular}

Table 10. Suture formula (Figs 13D, F-J), conch characteristics, and ornament of Taouzites taouzensis (Termier \& Termier, 1950) from the type locality.

$\left(E_{2} E_{1} E_{2}\right) L U_{2}: U_{1} l$ at $8 \mathrm{~mm}$ dm (MB.C.22106.15)

$\left(E_{2} E_{1} E_{2}\right) L U_{2} U_{4}: U_{3} U_{1} l$ at $9.5 \mathrm{~mm}$ dm (MB.C.22106.21)

$\left(E_{2} E_{1} E_{2}\right) L U_{2} U_{4} U_{5}: U_{3} U_{1} l$ at $18 \mathrm{~mm}$ dm (MB.C.22106.8)

$\left(E_{2} E_{1} E_{2}\right) L U_{2} U_{4} U_{5} U_{7}: U_{6} U_{3} U_{1} l$ at $30 \mathrm{~mm}$ dm (MB.C.22106.11)

$\left(\mathrm{E}_{2} \mathrm{E}_{1} \mathrm{E}_{2}\right) L \mathrm{U}_{2} \mathrm{U}_{4} \mathrm{U}_{5} \mathrm{U}_{7} \mathrm{U}_{8}: \mathrm{U}_{6} \mathrm{U}_{3} \mathrm{U}_{1} \mathrm{l}$ at $35 \mathrm{~mm}$ dm (MB.C.22106.7)

$E_{1}$ lobe broad, $V$ - or funnel-shaped, $\sim 50 \%$ depth of $E_{2}$ lobe; ventrolateral saddle broad and as high as median saddle

$\mathrm{L}$ lobe as deep or sometimes significantly deeper than $\mathrm{E}_{2}$; asymmetrical development of inner and outer $\mathrm{U}$ lobes from $\mathrm{U}_{5}$

venter oxyconic from 5-8 $\mathrm{mm} \mathrm{dm}$ on, umbilical width variable

juvenile ribs, ventrolateral furrows; no internal constrictions

growth lines rectiradiate, strongly biconvex, with high ventrolateral projection and deep lateral sinus

Suborder Pharciceratina Korn, 1998

Superfamily Pharcicerataceae Hyatt, 1900

Family Pharciceratidae Hyatt, 1900

\section{Subfamily Pharciceratinae Hyatt, 1900}

\section{Pharciceras Hyatt, 1884}

Type species. Goniatites tridens Sandberger \& Sandberger, 1850

\section{Pharciceras decoratum n. sp.}

Figures 15, 16

Derivation of name. After the decorative ribbing of early to median whorls.

Holotype. MB.C.22107.1 (Figs 15B and 16B). 

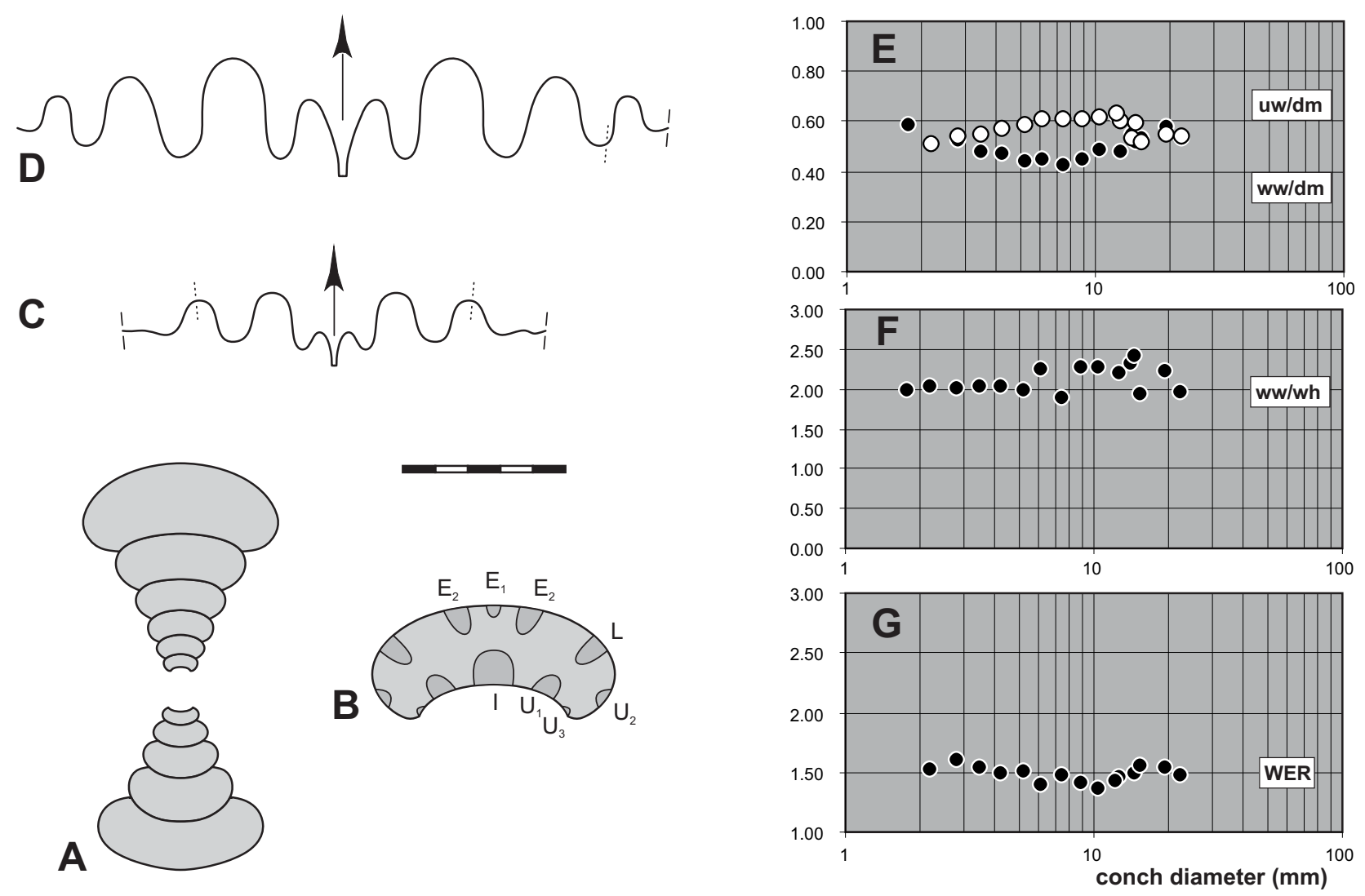

Figure 15. Pharciceras decoratum n. sp. from Hassi Nebech; A. Cross-section of paratype MB.C.22107.2, $\times 4$; B. Septal face of holotype MB.C.22107.1, $\times$ 4; C. Suture of paratype MB.C.22107.3 at $2.1 \mathrm{~mm}$ wh, $\times 4$; D. Suture of paratype MB.C.22107.4 at $4.9 \mathrm{~mm}$ wh, $\times 4$; E-G. Ontogenetic development of ww/dm, uw/dm, ww/wh, and WER of all available specimens.

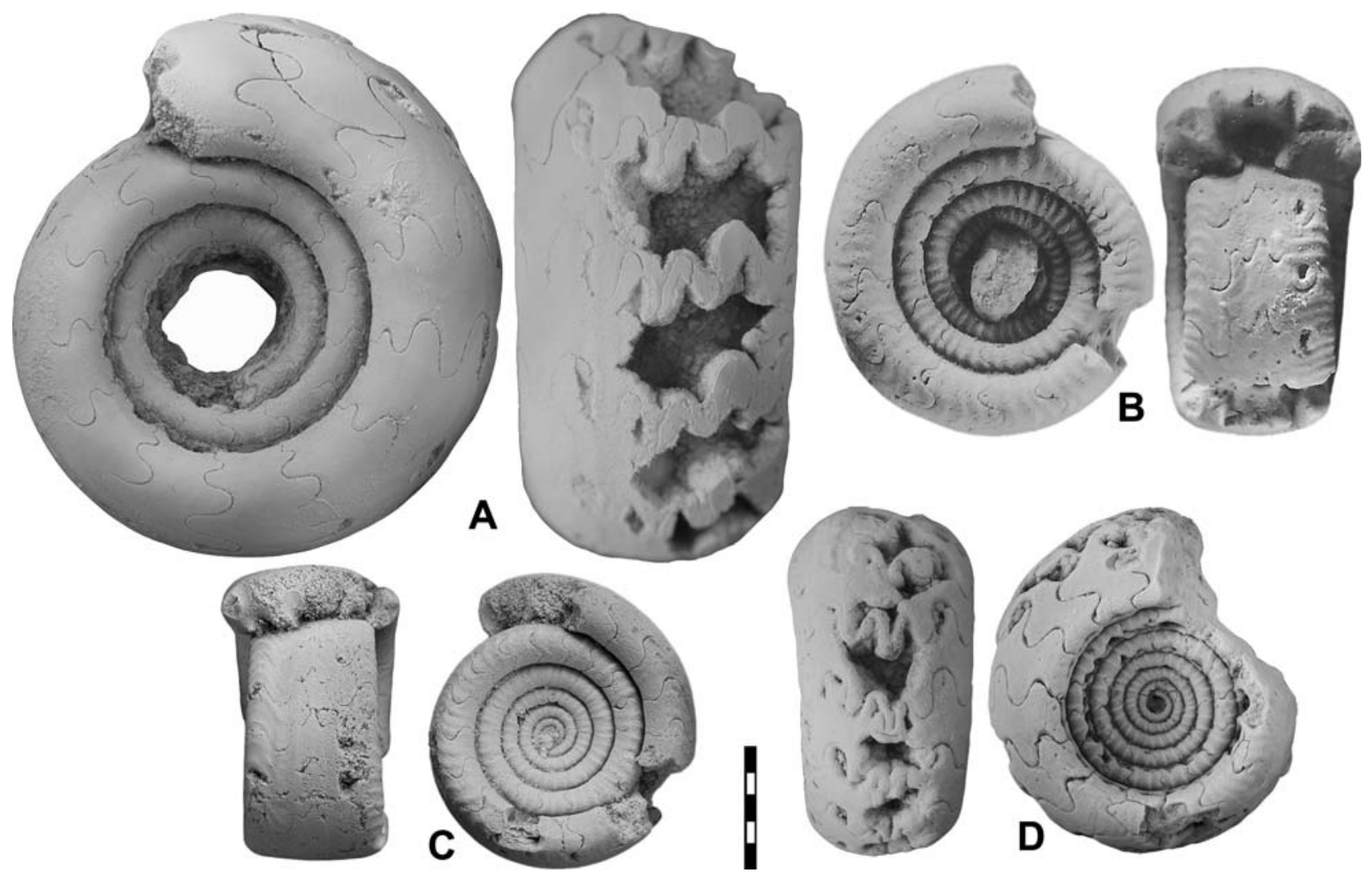

Figure 16. Pharciceras decoratum n. sp. from Hassi Nebech; A. Paratype MB.C.22107.4; B. Holotype MB.C.22107.1; C. Paratype MB.C.22107.3; D. MB.C.22107.7, more involute than typical specimens; all $\times 3$. 
Type locality and horizon. Hassi Nebech, Section 2, SE Tafilalt, main collecting level, probably Taouzites taouzensis Zone (late Givetian). Material. Seven specimens up to $22 \mathrm{~mm}$ conch diameter (MB.C.22107.1-MB.C.22107.7).

Diagnosis. Early to median stages with relatively uniform, sharply ribbed whorls, with slightly flattened venter and WER fluctuating between 1.40 and 1.60; ww/wh values vary between 2.00 and 2.50, post-embryonic ww/dm ratios first decrease from 0.60 to 0.40 (at ca. $7 \mathrm{~mm} \mathrm{dm}$ ) then rise back to 0.60 ; the umbilicus widens gradually until ca. $13 \mathrm{~mm}$ (uw/dm from 0.50 to $>0.60$ ) and becomes slightly narrower subsequently, with uw/dm $<$ ww/dm from $20 \mathrm{~mm} \mathrm{dm}$. Growth lines biconvex, with projecting ventrolateral salient in spiral double furrows. Sutures with diverging $\mathrm{E}_{1}$ lobe, narrow, moderately low median saddle, narrow $\mathrm{E}_{2}$ lobe, high and narrow $\mathrm{E}_{2} \mathrm{~L}$ saddle, deep L lobe, and two small outer and inner $\mathrm{U}$ lobes.

Table 11. Conch ontogeny (Figs 15A, B, E-G) of Pharciceras decoratum n. sp.

\begin{tabular}{|c|c|c|c|}
\hline$d m$ & conch shape & whorl cross-section shape & whorl expansion \\
\hline $2 \mathrm{~mm}$ & $\begin{array}{l}\text { thickly discoidal; evolute } \\
\text { (ww/dm } \sim 0.50 ; \text { uw/dm } \sim 0.50 \text { ) }\end{array}$ & $\begin{array}{l}\text { moderately depressed; moderately embracing } \\
\text { (ww/wh } \sim 2.00 ; \text { IZR } \sim 0.24 \text { ) }\end{array}$ & $\begin{array}{l}\text { low } \\
(\text { WER } \sim 1.50)\end{array}$ \\
\hline $5 \mathrm{~mm}$ & $\begin{array}{l}\text { thinly discoidal; evolute } \\
\text { (ww/dm } \sim 0.45 ; \text { uw/dm } \sim 0.60 \text { ) }\end{array}$ & $\begin{array}{l}\text { moderately depressed; moderately embracing } \\
\text { (ww/wh } \sim 2.00 ; \text { IZR } \sim 0.20 \text { ) }\end{array}$ & $\begin{array}{l}\text { low } \\
(\text { WER } \sim 1.50)\end{array}$ \\
\hline $10 \mathrm{~mm}$ & $\begin{array}{l}\text { thickly discoidal; evolute } \\
\text { (ww/dm } \sim 0.50 ; \text { uw/dm } \sim 0.60 \text { ) }\end{array}$ & $\begin{array}{l}\text { strongly depressed; moderately embracing } \\
\text { (ww/wh } \sim 2.30 ; \text { IZR } \sim 0.30 \text { ) }\end{array}$ & $\begin{array}{l}\text { low } \\
(\text { WER } \sim 1.35)\end{array}$ \\
\hline $20 \mathrm{~mm}$ & $\begin{array}{l}\text { thickly discoidal; evolute } \\
\text { (ww/dm } \sim 0.55 ; \text { uw/dm } \sim 0.55 \text { ) }\end{array}$ & $\begin{array}{l}\text { strongly depressed; moderately to strongly embracing } \\
\text { (ww/wh }=2.00-2.20 ; \mathrm{IZR}=0.25-0.35 \text { ) }\end{array}$ & $\begin{array}{l}\text { low } \\
(\text { WER }=1.50-1.55)\end{array}$ \\
\hline
\end{tabular}

Table 12. Suture formula (Figs 15B-D), conch characteristics, and ornament of Pharciceras decoratum n. sp.

$\left(E_{2} E_{1} E_{2}\right) L U_{2}: U_{3} U_{1} I$ at $14 \mathrm{~mm} d m(M B . C .22107 .1)$

$\left(E_{2} E_{1} E_{2}\right) L U_{2} U_{4}: U_{3} U_{1} l$ at $22 \mathrm{~mm} d m$ (MB.C.22107.4)

$\mathrm{E}_{1}$ lobe narrow, deep, diverging; ventrolateral saddle high, rounded; $L$ lobe deep and rounded

juvenile venter flat, in adults broadly rounded; umbilicus wide

inner whorls sharply ribbed; adult ventrolateral double furrows; no mould constrictions

growth lines biconvex with high ventrolateral salient

\section{Pharciceras aff. tridens (Sandberger \& Sandberger, 1850)}

Figures 17,18

Material. Seven specimens up to $24 \mathrm{~mm}$ conch diameter (MB.C.22108.1-MB.C.22108.7).

Table 13. Conch ontogeny (Figs 17A, B, D-F) of Pharciceras aff. tridens (Sandberger \& Sandberger, 1850).

\begin{tabular}{|c|c|c|c|}
\hline $\mathrm{dm}$ & conch shape & whorl cross-section shape & whorl expansion \\
\hline $2 \mathrm{~mm}$ & $\begin{array}{l}\text { thickly discoidal; evolute } \\
\text { (ww/dm } \sim 0.50 ; \text { uw/dm } \sim 0.45 \text { ) }\end{array}$ & $\begin{array}{l}\text { moderately depressed; moderately embracing } \\
\text { (ww/wh } \sim 1.85 ; \text { IZR } \sim 0.15 \text { ) }\end{array}$ & $\begin{array}{l}\text { low } \\
(\text { WER } \sim 1.70)\end{array}$ \\
\hline $5 \mathrm{~mm}$ & $\begin{array}{l}\text { thickly pachyconic; evolute } \\
\text { (ww/dm } \sim 0.50 ; \text { uw/dm } \sim 0.60 \text { ) }\end{array}$ & $\begin{array}{l}\text { strongly depressed; moderately embracing } \\
\text { (ww/wh } \sim 2.25 ; \text { IZR } \sim 0.20 \text { ) }\end{array}$ & $\begin{array}{l}\text { Low } \\
(\text { WER }=1.40-1.50)\end{array}$ \\
\hline $10 \mathrm{~mm}$ & $\begin{array}{l}\text { thickly pachyconic; evolute } \\
\text { (ww/dm } \sim 0.55 ; \text { uw/dm } \sim 0.60 \text { ) }\end{array}$ & $\begin{array}{l}\text { strongly depressed; moderately embracing } \\
\text { (ww/wh } \sim 2.45 ; \text { IZR } \sim 0.25 \text { ) }\end{array}$ & $\begin{array}{l}\text { low } \\
(\text { WER }=1.40-1.50)\end{array}$ \\
\hline $20 \mathrm{~mm}$ & $\begin{array}{l}\text { thickly discoidal; evolute } \\
\text { (ww/dm } \sim 0.55 ; \text { uw/dm } \sim 0.50 \text { ) }\end{array}$ & $\begin{array}{l}\text { moderately; moderately embracing } \\
\text { (ww/wh } \sim 2.00 ; \text { IZR } \sim 0.25 \text { ) }\end{array}$ & $\begin{array}{l}\text { low } \\
(\text { WER } \sim 1.65)\end{array}$ \\
\hline
\end{tabular}

Table 14. Suture formula (Figs 17B, C), conch characteristics, and ornament of Pharciceras. aff. tridens (Sandberger \& Sandberger, 1850).

$\left(E_{2} E_{1} E_{2}\right) L U_{2}: U_{1} l$ at $6 \mathrm{~mm} d m(M B . C .22108 .5)$

$\left(E_{2} E_{1} E_{2}\right) L_{2} U_{4}: U_{3} U_{1} l$ at $19 \mathrm{~mm} d m$ (MB.C.22108.3)

$E_{1}$ lobe narrow, deep; ventrolateral saddle high, rounded; L lobe broad and large

juvenile venter flat, in adults broadly rounded; umbilicus moderately wide

inner whorls distinctively ribbed; adult with shallow ventrolateral double furrows; no mould constrictions

growth lines strongly biconvex, with high ventrolateral salient 

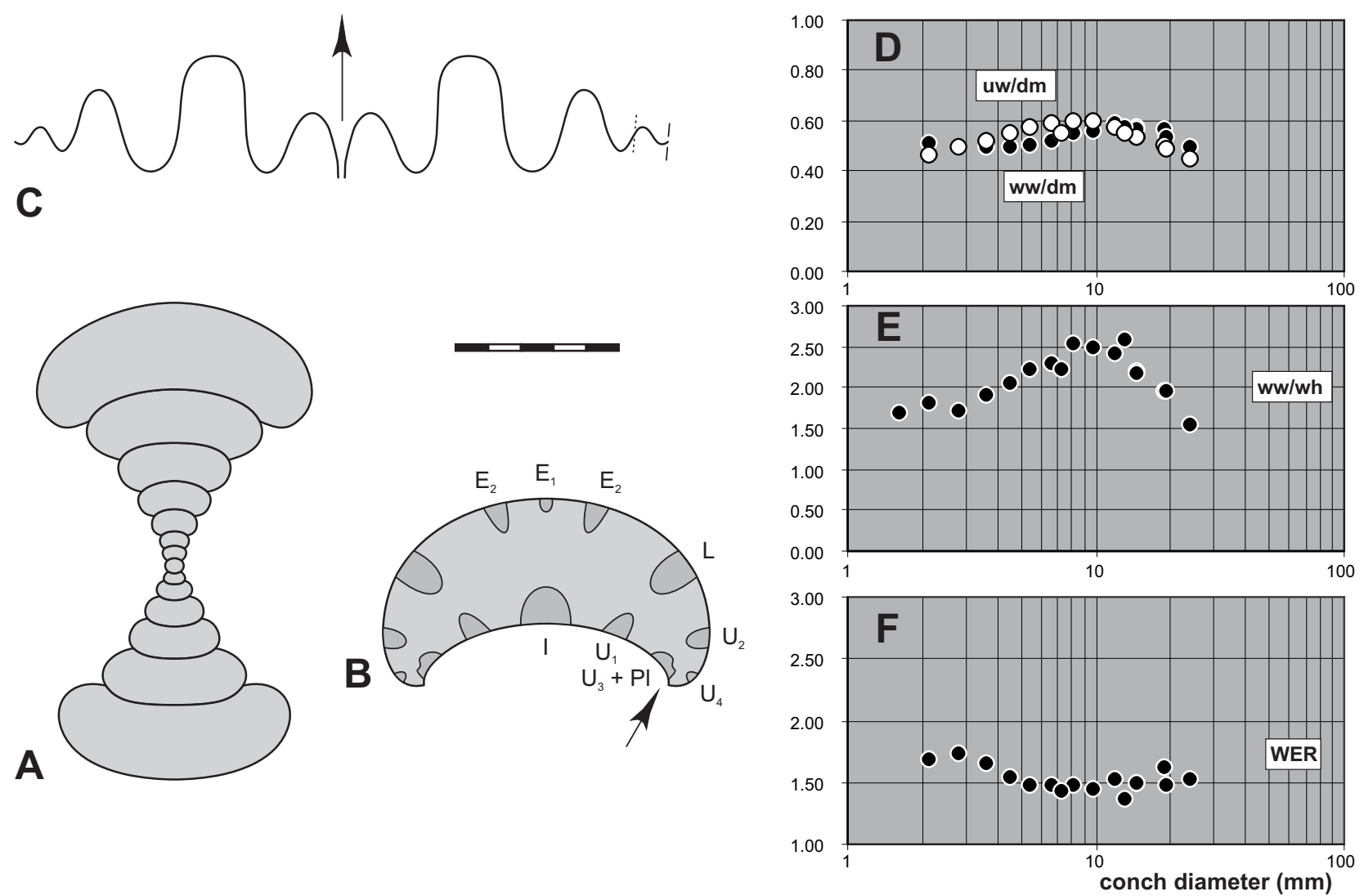

Figure 17. Pharciceras aff. tridens (Sandberger \& Sandberger, 1850) from Hassi Nebech; A. Cross-section of MB.C.22108.1, $\times 4$; B. Septal face of MB.C.22108.2, $\times 4$; C. Suture of MB.C.22108.3 at $5.5 \mathrm{~mm}$ wh, $\times 4$; D-F. Ontogenetic development of ww/dm, uw/dm, ww/wh, and WER of all available specimens.
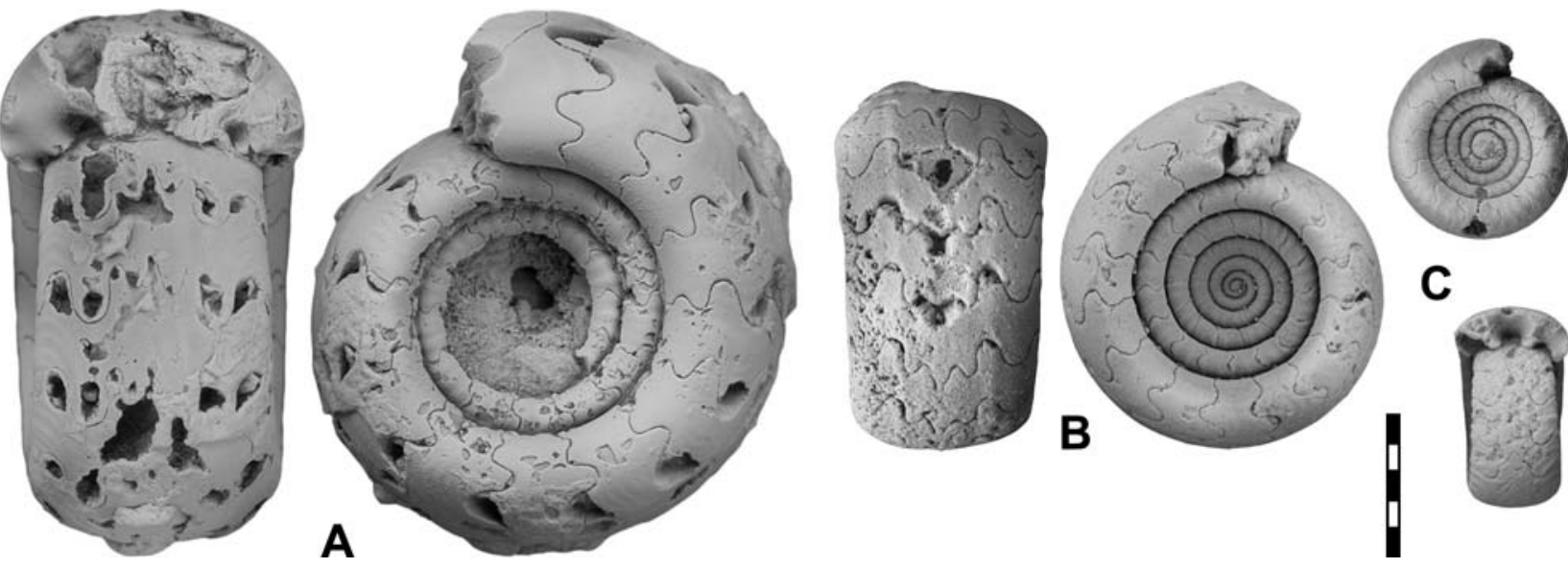

Figure 18. Pharciceras aff. tridens (Sandberger \& Sandberger, 1850) from Hassi Nebech; A. MB.C.22108.3; B. MB.C.22108.4; C. MB.C. 22108.5 ; all $\times 3$.

\section{Pharciceras lateseptatum Frech, 1902}

Figures 19-21

Lectotype. Original specimen figured by Frech (1902), designated in Bockwinkel et al. (2009), which, however, probably was lost at Breslau (Wroclaw) during the 2nd World War.

Material. Ca. 120 specimens up to $33 \mathrm{~mm}$ conch diameter, including MB.C.22109.1-MB.C.22109.25.

Diagnosis (emend.). Moderate-sized, first five whorls, ca. until $8 \mathrm{~mm} \mathrm{dm}$, increasingly depressed and evolute, with strongly increasing ww/wh (up to ca. 2.50), weakly increasing ww/dm (up to $0.60-0.80$ ) and rapid rise of uw/dm ratios (up to 0.45 to 0.50 ); WER decreases to $1.50-1.60$; subsequently less depressed, with decreasing relative umbilical width and rapidly expanding whorls; last whorl slightly compressed (ww/wh near $1.00 \mathrm{at}$ ca. $50 \mathrm{~mm}$ $\mathrm{dm}$ ) and subevolute (uw/dm 0.25 to 0.30 , adult WER $=1.60$ to 1.80 ). Early whorls markedly ribbed, subsequently with evenly spaced, strongly 
Table 15. Conch ontogeny (Figs 19A-D, F-H) of Pharciceras lateseptatum (Frech, 1902) Morphotype I from Hassi Nebech.

\begin{tabular}{|c|c|c|c|}
\hline$d m$ & conch shape & whorl cross-section shape & whorl expansion \\
\hline $2 \mathrm{~mm}$ & $\begin{array}{l}\text { thinly pachyconic; subevolute } \\
\text { (ww/dm } \sim 0.60 ; \text { uw/dm } \sim 0.45 \text { ) }\end{array}$ & $\begin{array}{l}\text { moderately depressed; moderately embracing } \\
\text { (ww/wh } \sim 2.00 ; \text { IZR } \sim 0.20 \text { ) }\end{array}$ & $\begin{array}{l}\text { low } \\
(\text { WER } \sim 1.70)\end{array}$ \\
\hline $5 \mathrm{~mm}$ & $\begin{array}{l}\text { thinly pachyconic; evolute } \\
\text { (ww/dm } \sim 0.65 ; \text { uw/dm } \sim 0.50 \text { ) }\end{array}$ & $\begin{array}{l}\text { strongly depressed; moderately embracing } \\
\text { (ww/wh } \sim 2.25 ; \text { IZR } \sim 0.30 \text { ) }\end{array}$ & $\begin{array}{l}\text { low } \\
(\text { WER } \sim 1.60)\end{array}$ \\
\hline $10 \mathrm{~mm}$ & $\begin{array}{l}\text { thinly pachyconic; subevolute } \\
\text { (ww/dm } \sim 0.65 ; \text { uw/dm } \sim 0.45 \text { ) }\end{array}$ & $\begin{array}{l}\text { strongly depressed; strongly embracing } \\
\text { (ww/wh } \sim 2.30 ; \text { IZR } \sim 0.30 \text { ) }\end{array}$ & $\begin{array}{l}\text { low } \\
(\text { WER } \sim 1.55)\end{array}$ \\
\hline $20 \mathrm{~mm}$ & $\begin{array}{l}\text { thinly pachyconic; subevolute } \\
\text { (ww/dm } \sim 0.60 \mathrm{uw} / \mathrm{dm} \sim 0.40 \text { ) }\end{array}$ & $\begin{array}{l}\text { moderately depressed; strongly embracing } \\
\text { (ww/wh }=1.50-2.00 ; \text { IZR } \sim 0.35 \text { ) }\end{array}$ & $\begin{array}{l}\text { low } \\
(\text { WER } \sim 1.75)\end{array}$ \\
\hline
\end{tabular}

Table 16. Conch ontogeny (Figs 21A, D-F) of Pharciceras lateseptatum (Frech, 1902) Morphotype II from Hassi Nebech.

\begin{tabular}{|c|c|c|c|}
\hline$d m$ & conch shape & whorl cross-section shape & whorl expansion \\
\hline $2 \mathrm{~mm}$ & $\begin{array}{l}\text { thinly pachyconic; subevolute } \\
\text { (ww/dm } \sim 0.60 ; \text { uw/dm } \sim 0.42 \text { ) }\end{array}$ & $\begin{array}{l}\text { strongly depressed; moderately embracing } \\
\text { (ww/wh } \sim 2.05 ; \text { IZR } \sim 0.20 \text { ) }\end{array}$ & $\begin{array}{l}\text { low } \\
(\text { WER } \sim 1.75)\end{array}$ \\
\hline $5 \mathrm{~mm}$ & $\begin{array}{l}\text { thickly pachyconic; subevolute } \\
\text { (ww/dm } \sim 0.75 ; \text { uw/dm } \sim 0.42 \text { ) }\end{array}$ & $\begin{array}{l}\text { strongly depressed; moderately embracing } \\
\text { (ww/wh } \sim 2.25 ; \text { IZR } \sim 0.30 \text { ) }\end{array}$ & $\begin{array}{l}\text { low } \\
(\text { WER } \sim 1.65)\end{array}$ \\
\hline $10 \mathrm{~mm}$ & $\begin{array}{l}\text { thickly pachyconic; subevolute } \\
\text { (ww/dm } \sim 0.75 ; \text { uw/dm } \sim 0.42\end{array}$ & $\begin{array}{l}\text { strongly depressed; strongly embracing } \\
\text { (ww/wh } \sim 2.50 \text {; IZR } \sim 0.35 \text { ) }\end{array}$ & $\begin{array}{l}\text { low } \\
(\text { WER } \sim 1.60)\end{array}$ \\
\hline $20 \mathrm{~mm}$ & $\begin{array}{l}\text { thinly pachyconic; subevolute } \\
\text { (ww/dm } \sim 0.70 ; \mathrm{uw} / \mathrm{dm} \sim 0.38 \text { ) }\end{array}$ & $\begin{array}{l}\text { moderately depressed; strongly embracing } \\
\text { (ww/wh } \sim 1.60 ; \text { IZR } \sim 0.35 \text { ) }\end{array}$ & $\begin{array}{l}\text { low } \\
(\text { WER } \sim 1.75)\end{array}$ \\
\hline
\end{tabular}

biconvex growth lirae and ventrolateral double furrows . Mature sutures with divergent $\mathrm{E}_{1}$ lobe, moderately high to high median saddle, deep, lanceolate $\mathrm{E}_{2}$ lobe, elevated and relative narrow ventrolateral saddle, moderately deep to deep, lanceolate $\mathrm{L}$ lobe, moderately narrow, asymmetric, pointed $\mathrm{U}_{2}$ lobe, wide, rounded to subangular $\mathrm{U}_{4}$ lobe, small internal $\mathrm{U}_{3}$ lobe, deep and narrow $\mathrm{U}_{1}$ lobe, and deep, narrow, lanceolate I lobe.
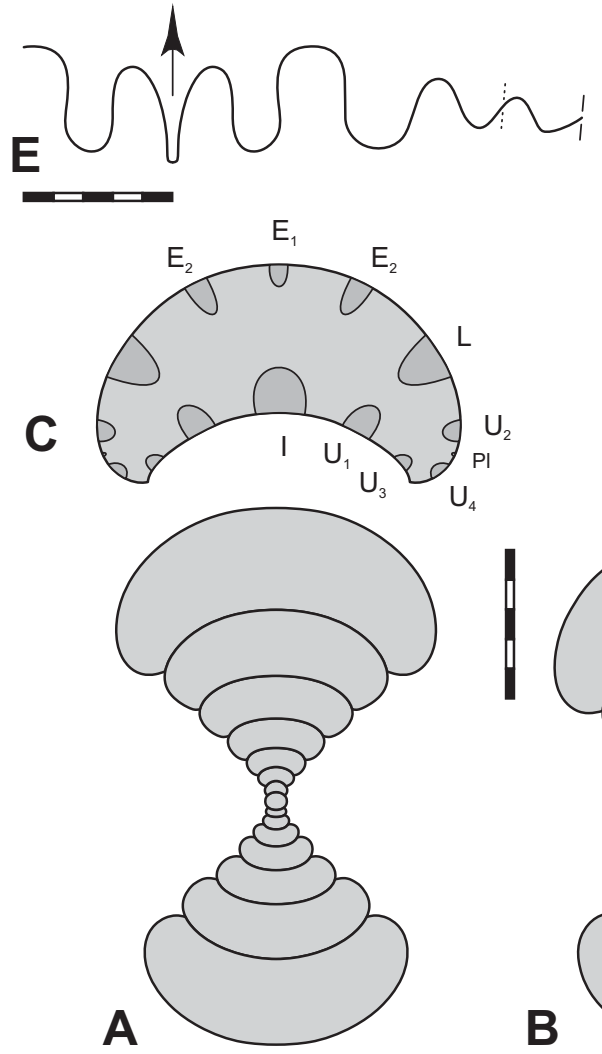
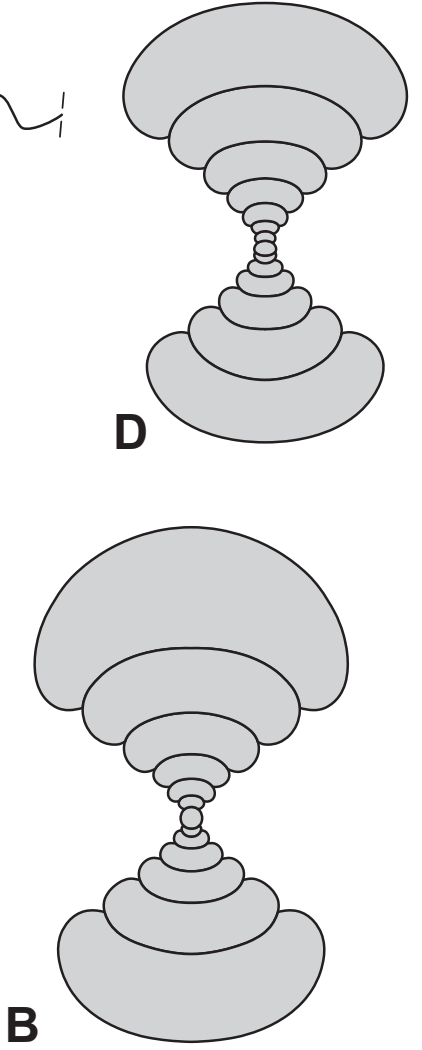

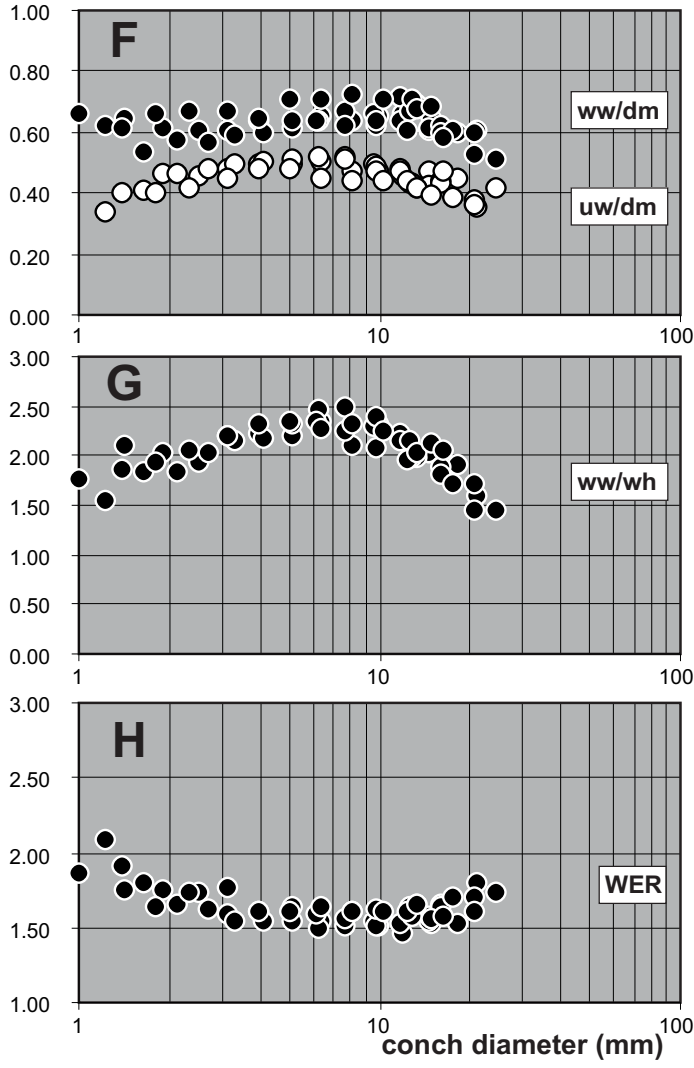

Figure 19. Pharciceras lateseptatum Frech, 1902 Morphotype I from Hassi Nebech; A. Cross-section of MB.C.22109.1, $\times 3$; B. Cross-section of MB.C.22109.2, ×3; C. Septal face of MB.C.22109.3, $\times 3$; D. Cross-section of MB.C.22109.4, $\times 3$; E. Suture of MB.C.22109.5 at $8.6 \mathrm{~mm} w \mathrm{w}, \times 3 ; \mathbf{F}-\mathbf{H}$. Ontogenetic development of ww/dm, uw/dm, ww/wh, and WER. 


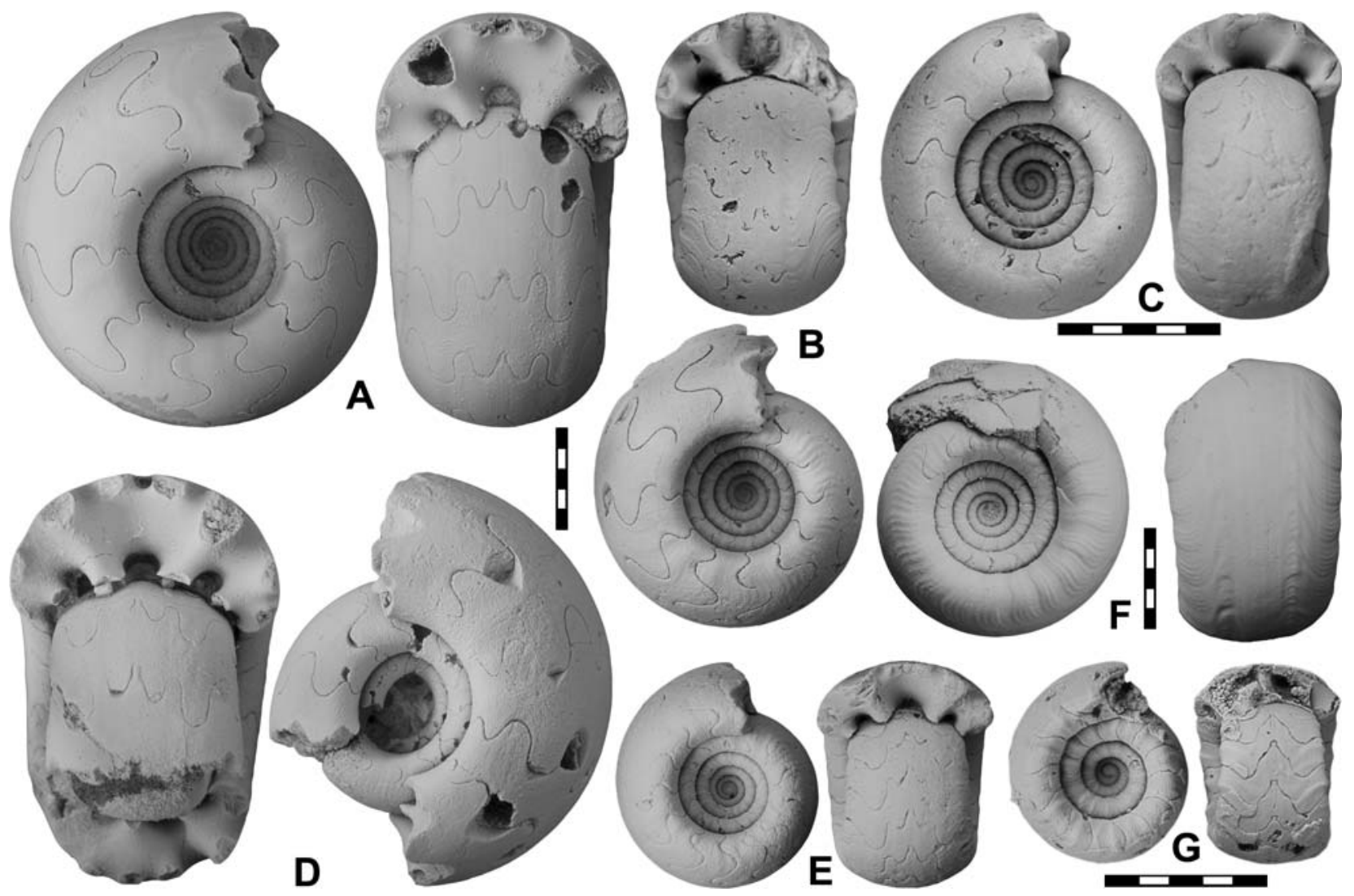

Figure 20. Pharciceras lateseptatum Frech, 1902 from Hassi Nebech; A-C. Morphotype I; A. MB.C.22109.3, ×2.5; B. MB.C. $22109.6, \times 2.5 ;$ C. MB.C.22109.7, $\times 4$; D-G. Morphotype II; D. MB.C.22109.8, $\times 2.5$; E. MB.C.22109.9, $\times 2.5$; F. MB.C. $22109.10, \times 2.5$; G. MB.C. $22109.11, \times 4$.
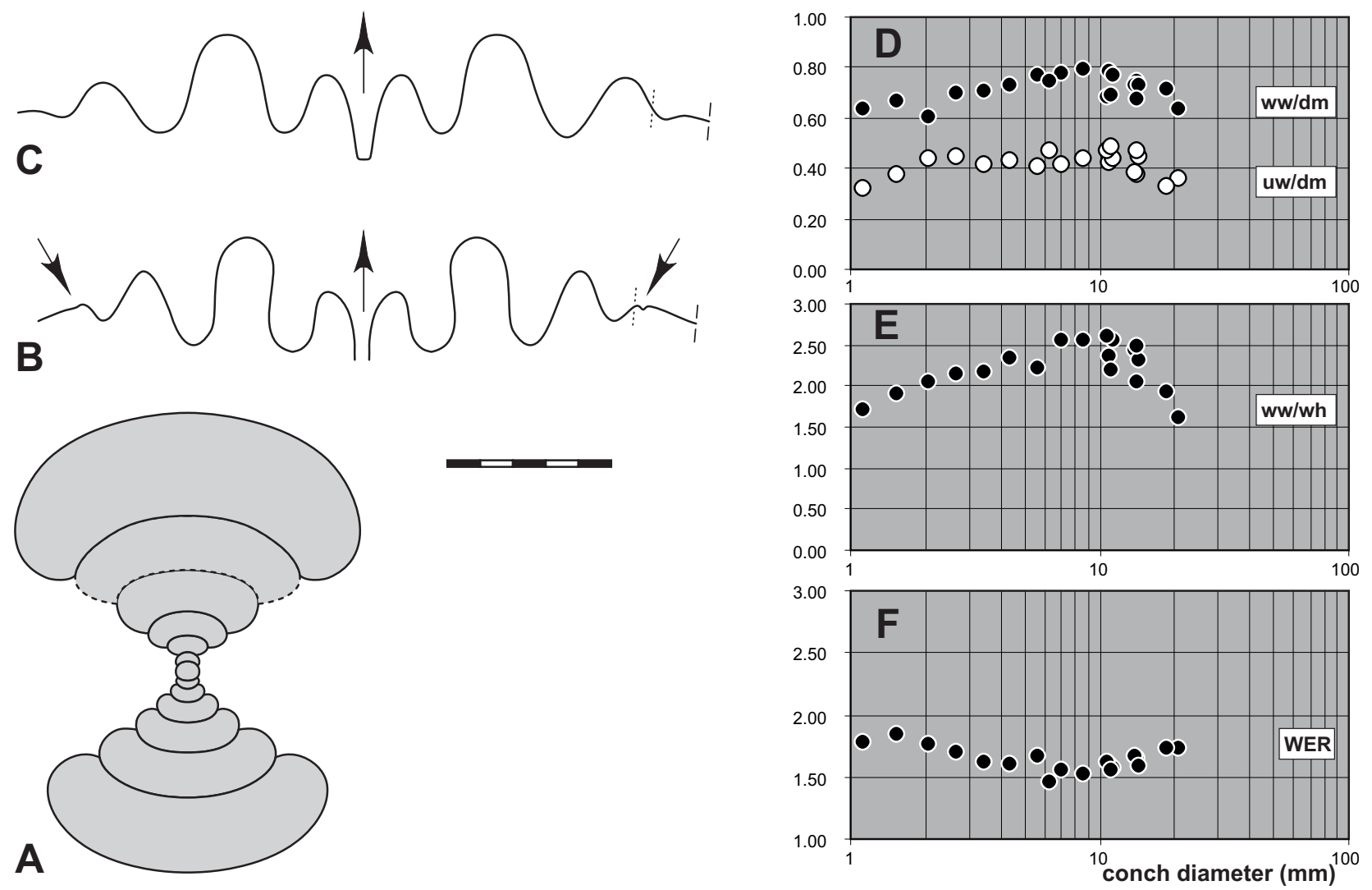

Figure 21. Pharciceras lateseptatum Frech, 1902 Morphotype II from Hassi Nebech; A. Cross-section of MB.C.22109.12, ×4; B. Suture of MB.C.22109.13 at $4.5 \mathrm{~mm}$ wh (arrows point at minor plurilobes), $\times 4$; C. Suture of MB.C.22109.12 at $4.8 \mathrm{~mm}$ wh, $\times 4$; D-F. Ontogenetic development of ww/dm, uw/dm, ww/wh, and WER. 
Table 17. Suture formula (Figs 19C, E, 21B, C), conch characteristics, and ornament of Pharciceras lateseptatum (Frech, 1902) from Hassi Nebech.

$\left(E_{2} E_{1} E_{2}\right) L U_{2}: U_{1} l$ at $6.5 \mathrm{~mm}$ dm (MB.C.22109.7, Morphotype I)

$\left(E_{2} E_{1} E_{2}\right) L U_{2}: U_{3} U_{1} l$ at $12 \mathrm{~mm}$ dm (MB.C.22109.17, Morphotype I)

$\left(E_{2} E_{1} E_{2}\right) L U_{2} U_{4}: U_{3} U_{1} l$ at $19 \mathrm{~mm}$ dm (MB.C.22109.3, Morphotype I)

$\mathrm{E}_{1}$ lobe narrow, deep, subparallel; ventrolateral saddle high, rounded; $L$ lobe broad and large venter of juvenile whorls flat, in adults broadly rounded

inner whorls with flank ribs; median whorls smooth or with shallow nodes, shallow ventrolateral double furrows, fading on moulds towards maturity; no internal constrictions

growth lines rectiradiate, strongly biconvex, with narrow and high projecting ventrolateral salient

Table 18. Comparison of typical ww/wh and uw/dm values in various Tafilalt Pharciceras species and morphotypes, with emphasis on Hassi Nebech representatives.

\begin{tabular}{|c|c|c|c|c|c|c|c|c|c|c|c|c|}
\hline & \multicolumn{6}{|l|}{ ww/wh } & \multicolumn{6}{|l|}{$\mathrm{uw} / \mathrm{dm}$} \\
\hline & $5 \mathrm{~mm}$ & $10 \mathrm{~mm}$ & $15 \mathrm{~mm}$ & $20 \mathrm{~mm}$ & $30 \mathrm{~mm}$ & $40 \mathrm{~mm}$ & $5 \mathrm{~mm}$ & $10 \mathrm{~mm}$ & $15 \mathrm{~mm}$ & $20 \mathrm{~mm}$ & $30 \mathrm{~mm}$ & $40 \mathrm{~mm}$ \\
\hline evolvens & 2.20 & 2.50 & 1.90 & $\begin{array}{l}1.80- \\
1.90\end{array}$ & 1.50 & 1.40 & 0.56 & 0.60 & 0.58 & 0.54 & 0.50 & 0.47 \\
\hline decoratum n. sp. & 2.00 & 2.30 & $\begin{array}{l}2.00- \\
2.50\end{array}$ & $\begin{array}{l}2.00- \\
2.30\end{array}$ & & & 0.60 & 0.60 & $\begin{array}{l}0.55- \\
0.60\end{array}$ & 0.55 & & \\
\hline aff. tridens & 2.20 & 2.50 & 2.20 & 1.90 & $<1.50$ & & 0.58 & 0.60 & 0.55 & 0.48 & $? 0.40$ & \\
\hline tridens & 2.30 & $\begin{array}{l}2.50- \\
2.70\end{array}$ & $\begin{array}{l}2.30- \\
2.50\end{array}$ & 2.20 & $\begin{array}{l}1.60- \\
1.80\end{array}$ & 1.40 & 0.50 & $\begin{array}{l}0.55- \\
0.60\end{array}$ & $\begin{array}{l}0.50- \\
0.55\end{array}$ & 0.50 & 0.45 & 0.40 \\
\hline lateseptatum Morphotype I & 2.30 & $\begin{array}{l}2.10- \\
2.30\end{array}$ & $\begin{array}{l}1.80- \\
2.20\end{array}$ & $\begin{array}{l}1.50- \\
1.60\end{array}$ & 1.30 & $\begin{array}{l}1.10- \\
1.20\end{array}$ & 0.50 & $\begin{array}{l}0.43- \\
0.48\end{array}$ & $\begin{array}{l}0.42- \\
0.45\end{array}$ & $\begin{array}{l}0.37- \\
0.40\end{array}$ & $\begin{array}{l}0.35- \\
0.37\end{array}$ & $\begin{array}{l}0.27- \\
0.32\end{array}$ \\
\hline lateseptatum Morphotype II & 3.20 & $\begin{array}{l}2.40- \\
2.60\end{array}$ & $\begin{array}{l}2.30- \\
2.40\end{array}$ & 1.90 & & & 0.50 & 0.45 & $\begin{array}{l}0.40- \\
0.45\end{array}$ & 0.37 & $\begin{array}{l}0.35- \\
0.37\end{array}$ & \\
\hline fornix n. sp. & $\begin{array}{l}2.20- \\
2.40\end{array}$ & $\begin{array}{l}2.00- \\
2.20\end{array}$ & $\begin{array}{l}1.70- \\
2.00\end{array}$ & 1.50 & 1.30 & & 0.41 & $\begin{array}{l}0.38- \\
0.41\end{array}$ & $\begin{array}{l}0.31- \\
0.37\end{array}$ & $\begin{array}{l}0.27- \\
0.33\end{array}$ & $\begin{array}{l}0.29- \\
0.32\end{array}$ & \\
\hline darkaouense & 2.01 & 1.80 & 1.60 & 1.55 & 1.30 & 1.15 & 0.40 & 0.41 & 0.39 & 0.38 & 0.37 & 0.35 \\
\hline pargai Morphotype I & 1.65 & 1.78 & 1.50 & 1.40 & 1.10 & & 0.42 & 0.42 & 0.36 & 0.33 & 0.31 & 0.28 \\
\hline pargai Morphotype II & 2.00 & $\begin{array}{l}1.70- \\
1.80\end{array}$ & $\begin{array}{l}1.50- \\
1.60\end{array}$ & $\begin{array}{l}1.20- \\
1.40\end{array}$ & $\begin{array}{l}1.10- \\
1.20\end{array}$ & 1.00 & 0.40 & $\begin{array}{l}0.38- \\
0.40\end{array}$ & $\begin{array}{l}0.28- \\
0.35\end{array}$ & $\begin{array}{l}0.25- \\
0.30\end{array}$ & $\begin{array}{l}0.22- \\
0.26\end{array}$ & $\begin{array}{l}0.22- \\
0.25\end{array}$ \\
\hline subconstans n. sp. & 1.90 & $\begin{array}{l}1.70- \\
1.80\end{array}$ & 1.70 & & & & 0.27 & 0.25 & 0.27 & & & \\
\hline
\end{tabular}

\section{Pharciceras fornix n. sp.}

Figures 22, 23

Derivation of name. After the Latin fornix = rounded hall; due to the well-rounded conch.

Holotype. MB.C.22110.1 (Figs 22H and 23B).

Material. Ca. 50 specimens up to $35 \mathrm{~mm}$ conch diameter, including MB.C.22110.1-MB.C.22110.13, and paratype STIPB-Bensaïd-29 (old: GPI Bo 29), original of Ph. cf. applanatum in Bensaïd, 1974, pl. 3, fig. 8, here re-illustrated in Fig. 23D.

Table 19. Conch ontogeny (Figs 22A-D, I-K) of Pharciceras fornix n. sp.

\begin{tabular}{llll}
\hline $\mathrm{dm}$ & conch shape & whorl cross-section shape & whorl expansion \\
\hline $2 \mathrm{~mm}$ & thinly pachyconic; subevolute & moderately depressed; strongly embracing & moderate \\
& $($ ww/dm $\sim 0.65 ;$ uw/dm $\sim 0.35)$ & $($ ww/wh $\sim 1.90 ;$ IZR $\sim 0.35)$ & (WER $\sim 1.80)$
\end{tabular}


Table 20. Suture formula (Figs 22C-H), conch characteristics, and ornament of Pharciceras fornix n. sp.

\section{$\left(E_{2} E_{1} E_{2}\right) L U_{2}: U_{3} U_{1} l$ at $13 \mathrm{~mm} d m(M B . C .22110 .12)$}

\section{$\left(E_{2} E_{1} E_{2}\right) L U_{2} U_{4}: U_{3} U_{1} l$ at $30 \mathrm{~mm} d m$ (MB.C.22110.9)}

$\mathrm{E}_{1}$ lobe deep, funnel-shaped; $\mathrm{E}_{2}$ lobe in adults as deep as $\mathrm{E}_{1}$; ventrolateral saddle high and broadly rounded, slightly asymmetric;

L lobe wide, moderately deep

venter broadly rounded; umbilicus moderately wide

juvenile ribs; ventrolateral double furrows; no mould constrictions

growth lines strongly biconvex, with high ventrolateral salient

Type locality and horizon. Hassi Nebech, Section 2, SE Tafilalt, main collecting level, probably Taouzites taouzensis Zone (late Givetian).

Diagnosis. First five whorls, until ca. $8.5 \mathrm{~mm} \mathrm{dm}$, ribbed and increasingly cadiconic, with rising whorl width (ww/dm up to 0.78 , ww/wh up to 2.40) and umbilical width rates (uw/dm up to 0.40 ); WER values drop to $1.50-1.60$; intermediate and mature stages gradually less depressed $(\mathrm{ww} / \mathrm{wh}=1.50$ at $20 \mathrm{~mm} \mathrm{dm})$ and subevolute/subinvolute $(\mathrm{uw} / \mathrm{dm}=0.27$ to 0.32$)$, slightly faster expanding (WER $=1.70-1.80)$, smooth.
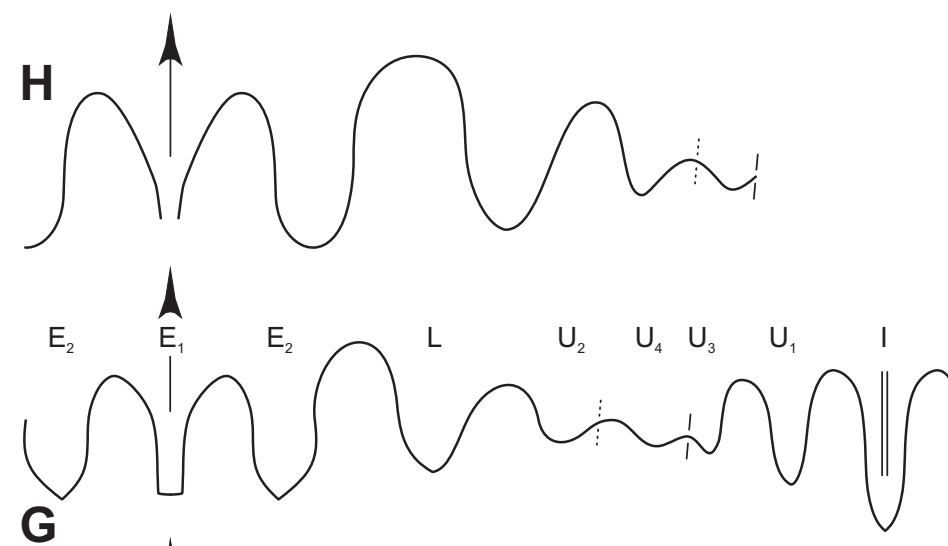

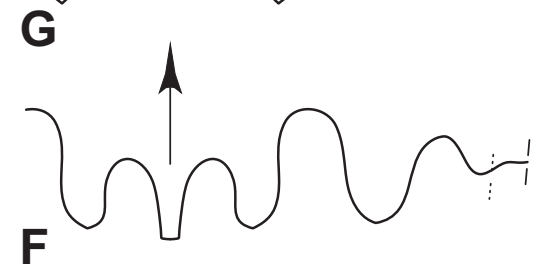

$\mathbf{F}$

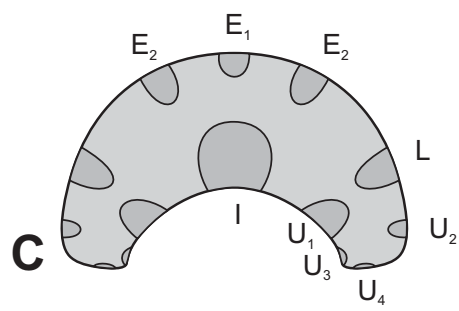

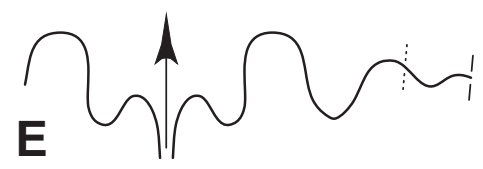
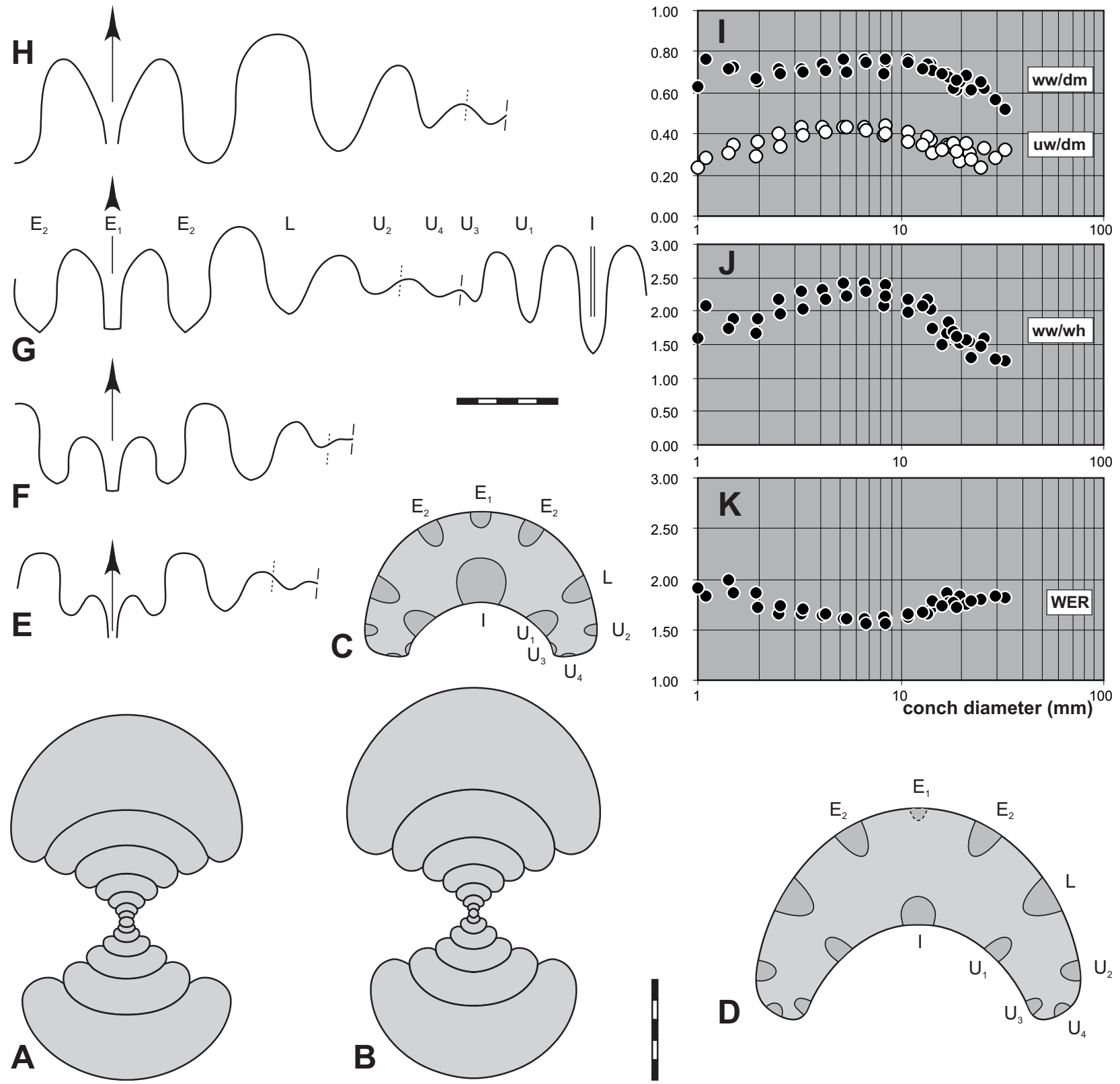

Figure 22. Pharciceras fornix n. sp. from Hassi Nebech; A. Cross-section of paratype MB.C.22110.11, $\times 3$; B. Cross-section of paratype MB.C.22110.2, ×3; C. Septal face of paratype MB.C.22110.3, ×3; D. Septal face of paratype MB.C.22110.4, ×3; E. Suture of paratype MB.C.22110.5 at $5.4 \mathrm{~mm}$ wh, $\times 3$; F. Suture of paratype MB.C.22110.6 at $6.1 \mathrm{~mm}$ wh, $\times 3$; G. Suture of paratype MB.C.22110.4 at $10.5 \mathrm{~mm}$ wh, $\times 3$; H. Suture of holotype MB.C.22110.1 at $16.5 \mathrm{~mm}$ wh, $\times 3$; I-K. Ontogenetic development of ww/dm, uw/dm, ww/wh, and WER. 

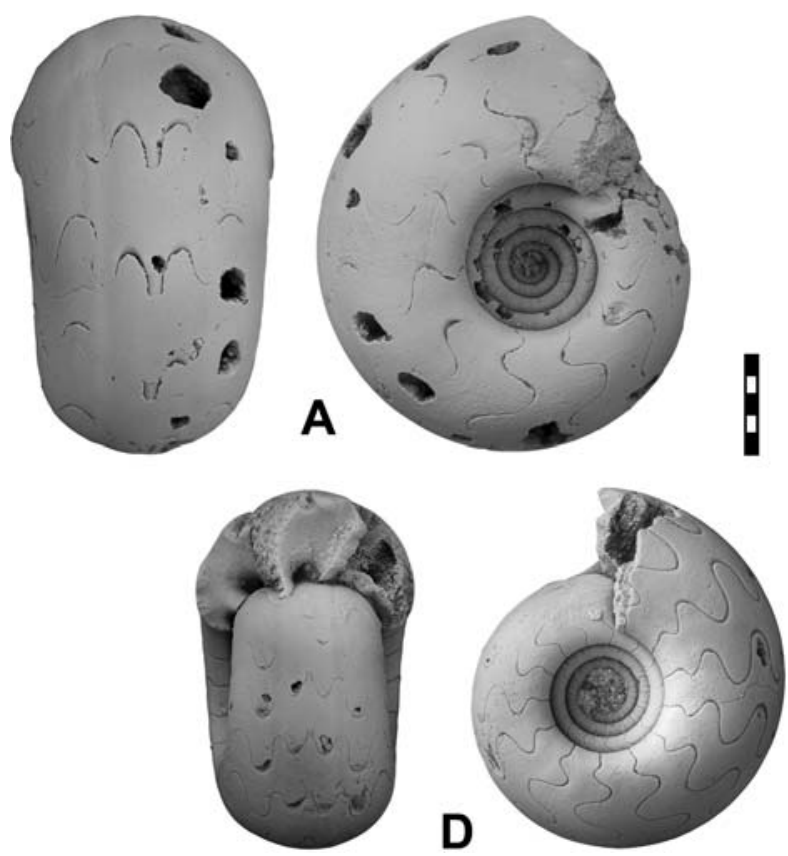

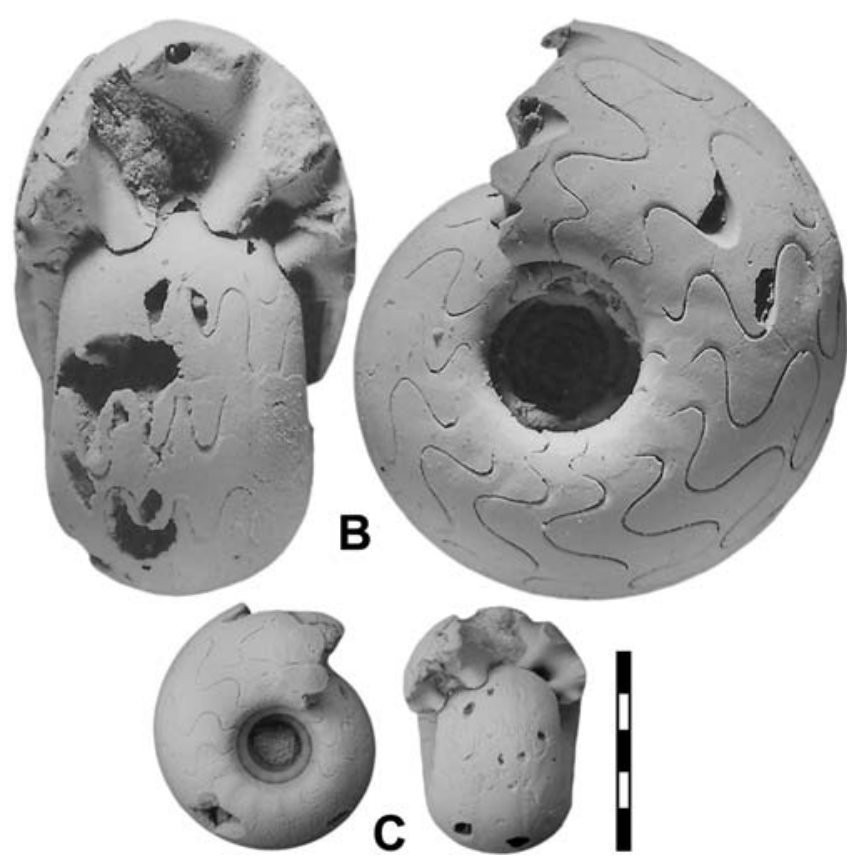

Figure 23. Pharciceras fornix n. sp. from Hassi Nebech; A. Paratype MB.C.22110.7, $\times 2$; B. Holotype MB.C.22110.1, $\times 2$; C. Paratype MB.C.22110.8, ×4; D. STIPB-Bensaïd-29 (old: GPI Bo 29), original of Ph. cf. applanatum of Bensaïd (1974, pl. 3, figs $8,8 \mathrm{a}), \times 2$.

Growth lines strongly biconvex, with narrow ventrolateral salient lying in spiral double furrows, which fade in intermediate stages. Mature sutures with narrow, slightly funnel-shaped $\mathrm{E}_{1}$ lobe, moderately high median saddle, deeply rounded or lanceolate $\mathrm{E}_{2}$ lobe, high $\mathrm{E}_{2} \mathrm{~L}$ saddle, pointed, divergent, moderately deep $L$ lobe, two rounded outer $U$ lobes separated by gradually lower, rounded saddles, small internal $U_{3}$ lobe, deep and narrow $\mathrm{U}_{1}$ lobe, and very deep, narrow, lanceolate I lobe.

\section{Pharciceras subconstans n. sp.}

Figures 24A, D-G, 25A, cf. 24B, C, 25B

Derivation of name. Due to its much more constant conch parameters throughout ontogeny than in most other Pharciceras species.

Holotype. MB.C.22111.1, which has been sectioned (Fig. 24A).

Type locality and horizon. Hassi Nebech, Section 2, SE Tafilalt, main collecting level, probably Taouzites taouzensis Zone (late Givetian).

Material: The holotype, paratype MB.C.22111.2, two specimens identified as cf. subconstans (MB.C.22112.1-MB.C.22112.2).

Diagnosis. First ca. 4 whorls until $10 \mathrm{~mm} \mathrm{dm}$ thickly pachyconic, with nearly constant ww/dm near 0.75 and slightly falling ww/wh ratios (from ca. 2.00 to $1.60-1.70$ ); uw/dm decreases from 0.35 to ca. 0.25 ; subsequently with little change of whorl width ratio and WER (1.70 to 1.80 ); the umbilicus re-opens slightly. Sutures until $15 \mathrm{~mm} \mathrm{dm}$ with small, divergent $\mathrm{E}_{1}$ lobe, small median saddle, narrow $\mathrm{E}_{2}$ lobe, almost as deep as the $\mathrm{E}_{1}$, high $\mathrm{E}_{2} \mathrm{~L}$ saddle, wide, deep L lobe, low dorsolateral saddle, small subumbilical $\mathrm{U}_{2}$ lobe, shallow $\mathrm{U}_{3}$ lobe just inside the umbilical seam, deep $\mathrm{U}_{1}$ and I lobe.

Table 21. Conch ontogeny (Figs 24A, B, E-G) of Pharciceras subconstans n. sp.

\begin{tabular}{|c|c|c|c|}
\hline $\mathrm{dm}$ & conch shape & whorl cross-section shape & whorl expansion \\
\hline $2 \mathrm{~mm}$ & $\begin{array}{l}\text { thickly pachyconic; subevolute } \\
\text { (ww/dm } \sim 0,75 ; \text { uw/dm } \sim 0.34 \text { ) }\end{array}$ & $\begin{array}{l}\text { moderately depressed; moderately embracing } \\
\text { (ww/wh } \sim 1.90 ; \text { IZR } \sim 0.30 \text { ) }\end{array}$ & $\begin{array}{l}\text { moderate } \\
(\text { WER } \sim 1.90)\end{array}$ \\
\hline $5 \mathrm{~mm}$ & $\begin{array}{l}\text { thickly pachyconic; subinvolute } \\
\text { (ww/dm } \sim 0.75 ; \text { uw/dm } \sim 0.27 \text { ) }\end{array}$ & $\begin{array}{l}\text { moderately depressed; strongly embracing } \\
\text { (ww/wh } \sim 1.80 ; \text { IZR } \sim 0.35 \text { ) }\end{array}$ & $\begin{array}{l}\text { moderate } \\
(\text { WER } \sim 1.80)\end{array}$ \\
\hline $15 \mathrm{~mm}$ & $\begin{array}{l}\text { thinly pachyconic; subinvolute } \\
\text { (ww/dm } \sim 0.70 ; \text { uw/dm } \sim 0.27 \text { ) }\end{array}$ & $\begin{array}{l}\text { moderately depressed; strongly embracing } \\
\text { (ww/wh }=1.50-1.60 ; \text { IZR } \sim 0.40 \text { ) }\end{array}$ & $\begin{array}{l}\text { low } \\
(\text { WER } \sim 1.70)\end{array}$ \\
\hline $20 \mathrm{~mm}$ & $\begin{array}{l}\text { thinly pachyconic; subevolute } \\
\text { (ww/dm } \sim 0.65 ; \text { uw/dm } \sim 0.35 \text { ) }\end{array}$ & $\begin{array}{l}\text { moderately depressed; strongly embracing } \\
\text { (ww/wh }=1.50-1.60 ; \text { IZR } \sim 0.40 \text { ) }\end{array}$ & $\begin{array}{l}\text { moderate } \\
(\text { WER } \sim 1.70)\end{array}$ \\
\hline
\end{tabular}


Table 22. Suture formula (Figs 24B-D), conch characteristics, and ornament of Pharciceras subconstans n. sp.

$\left(E_{2} E_{1} E_{2}\right) L U_{2}: U_{3} U_{1} l$ at $10 \mathrm{~mm}$ dm (cf. MB.C.22112.1)

\section{$\left(E_{2} E_{1} E_{2}\right) L U_{2}: U_{3} U_{1} I$ at $15 \mathrm{~mm}$ dm (holotype)}

$E_{1}$ lobe funnel-shaped, deep, narrow; ventrolateral saddle high, broadly rounded, symmetric; L lobe broad, slightly asymmetric venter broadly rounded, umbilicus moderately narrow no mould constrictions; shallow ventrolateral furrows growth lines rectiradiate, biconvex, with high ventrolateral projection
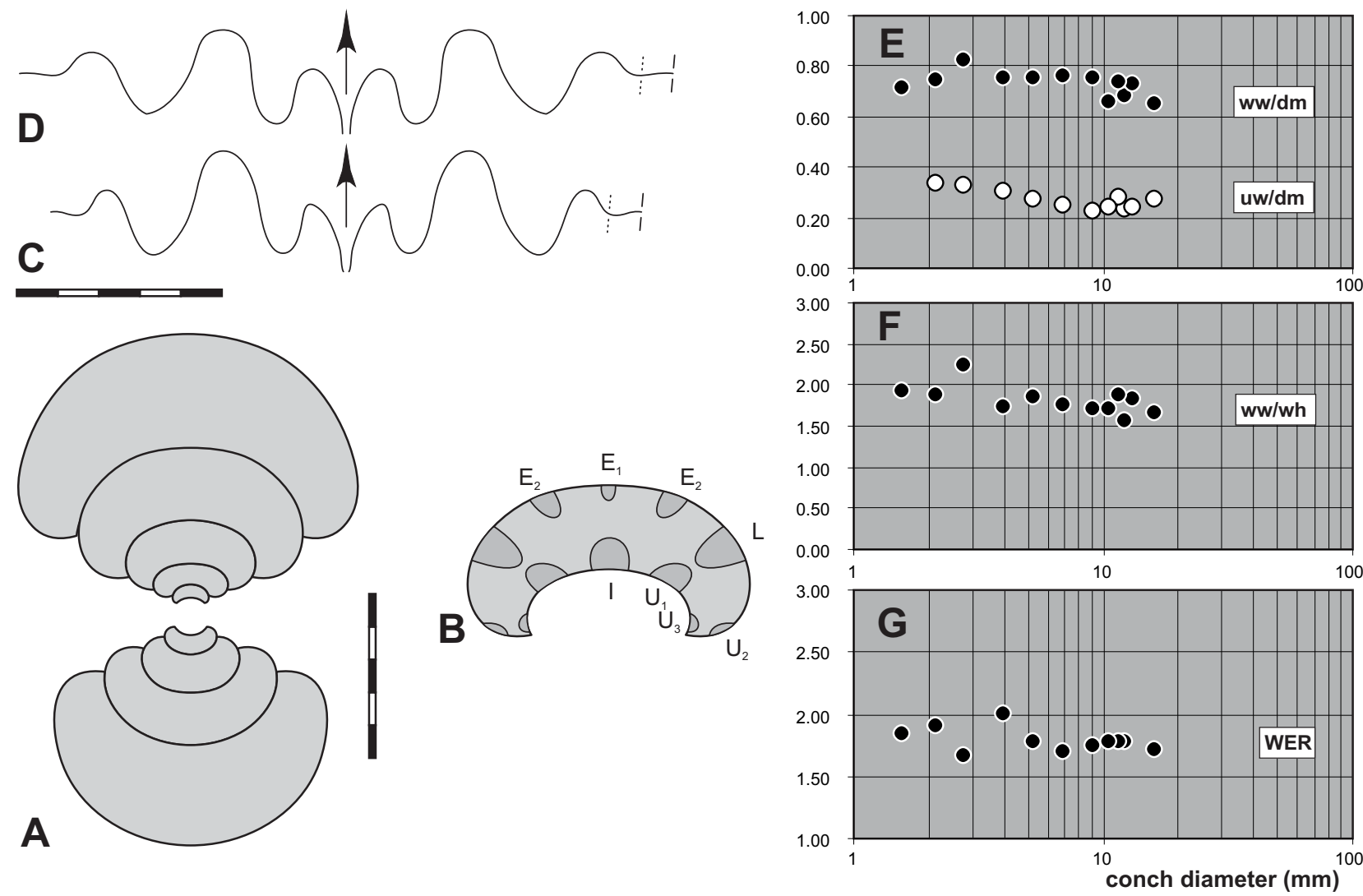

Figure 24. Pharciceras subconstans n. sp., including a cf. specimen, from Hassi Nebech; A. Cross-section of holotype MB.C.22111.1, $\times 4$; B. Septal face of cf. specimen, MB.C.22112.1, $\times 4$; C. Suture of cf. specimen MB.C.22112.1 at $3.2 \mathrm{~mm}$ wh, $\times 5$; D. Suture of paratype MB.C.22111.2 at $4.1 \mathrm{~mm} w h, \times 5 ; \mathbf{E}-$ G. Ontogenetic development of ww $/ \mathrm{dm}, \mathrm{uw} / \mathrm{dm}$, ww/wh, and WER of the holotype and paratype.

\section{Pharciceras involutum n. sp.}

\section{Figures $25 \mathrm{C}, 26$}

Derivation of name. Due to the involute conch. Holotype. MB.C.22113 (Figs 25C, 26A, B).

Material. Only the holotype, which was cast before sectioning.

Type locality and horizon. Hassi Nebech, Section 2, SE Tafilalt, nodular level in the upper part of Bed 11, probably Taouzites taouzensis Zone (late Givetian).

Table. 23. Conch ontogeny (Fig. 26A) of Pharciceras involutum n. sp.

\begin{tabular}{llll}
\hline $\mathrm{dm}$ & conch shape & whorl cross-section shape & whorl expanion \\
\hline $20 \mathrm{~mm}$ & thinly discoidal; involute & weakly compressed; strongly embracing & high \\
& $($ ww/dm $\sim 0.42)$ & (ww/wh $\sim 0,90 ;$ IZR $\sim 0,35)$ & $($ WER $\sim 2.10)$ \\
$30 \mathrm{~mm}$ & thinly discoidal; involute & weakly compressed; strongly embracing & high \\
& $($ ww/dm $\sim 0.38 ;$ uw/dm $\sim 0.15)$ & $($ ww/wh $\sim 0,75 ;$ IR $\sim 0,40)$ & $($ WER $\sim 2.05)$ \\
$44 \mathrm{~mm}$ & extremly discoidal; involute & weakly compressed; very strongly embracing & moderate \\
& $($ ww/dm $\sim 0.33 ;$ uw/dm $\sim 0.15)$ & $($ ww/wh $\sim 0,70 ;$ IZR $\sim 0,45)$ & $($ WER $\sim 1,90)$ \\
\hline
\end{tabular}


Table 24. Suture formula (Fig. 26B), conch characteristics, and ornament of Pharciceras involutum n. sp.

$\left(E_{2} E_{1} E_{2}\right) L U_{2} U_{4}$ : probably $U_{3} U_{1} l$ at $32 \mathrm{~mm} \mathrm{dm}$

$E_{1}$ lobe deep, narrow, funnel-shaped; wide median saddle, narrow $E_{2}$ lobe, short $L$ lobe narrow venter narrowly rounded, involute strong ventrolateral furrows; no ribs; a single internal constriction growth lines not visible

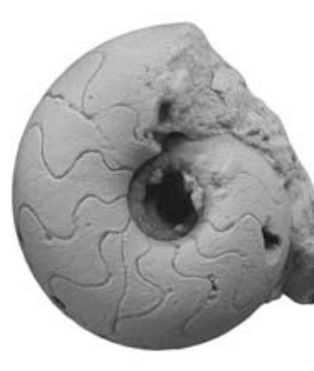

A

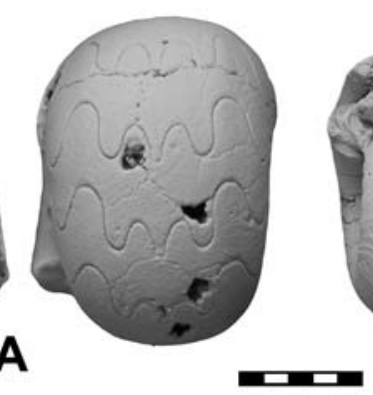

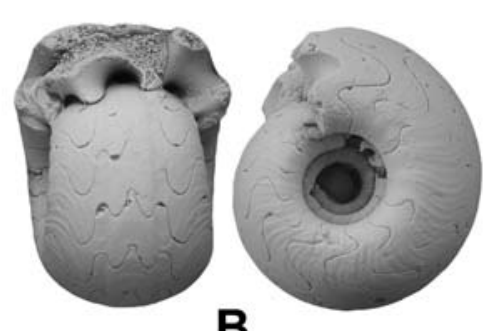

B

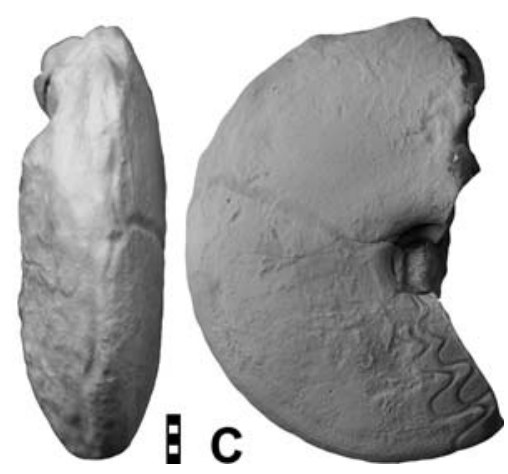

Figure 25. A. Pharciceras subconstans n. sp. from Hassi Nebech; paratype MB.C.22111.2, $\times 2.5$; B. Pharciceras cf. subconstans n. sp. from Hassi Nebech; MB.C.22112.1, ×2.5; C. Pharciceras involutum n. sp. from Hassi Nebech; Holotype MB.C.22113, $\times 1$.

Diagnosis. Mature conch compressed $(\mathrm{ww} / \mathrm{dm}=\mathrm{ca} .0 .33)$, involute $(\mathrm{uw} / \mathrm{dm}=\mathrm{ca} .0 .15)$, with gently rounded flanks and venter, separated by a marked ventrolateral edge and a deep, narrow spiral furrow. Mature suture with narrow, funnel-shaped $\mathrm{E}_{1}$ lobe, prominent, relatively wide median saddle, narrow, tongue-shaped $E_{2}$ lobe, slightly deeper than the $E_{1}$, moderately wide, high, slightly asymmetric ventrolateral saddle, distinctively short, narrow $\mathrm{L}$ lobe, and two small, wide outer $\mathrm{U}$ lobes separated by a wide and low saddle.

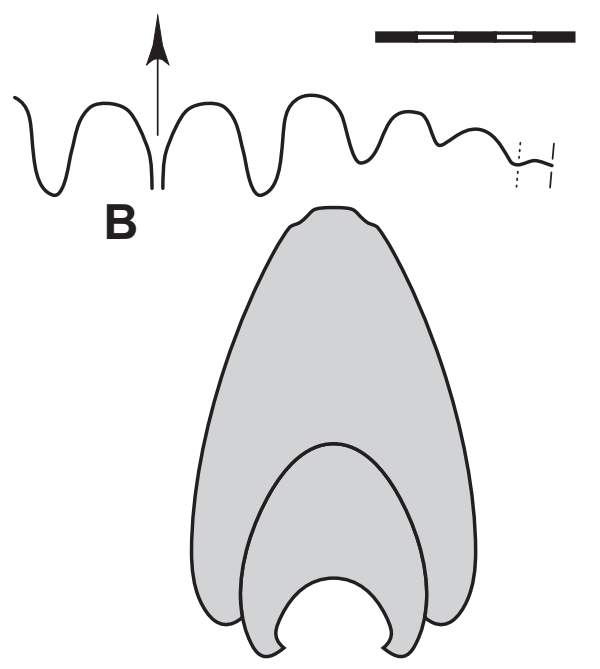

Figure 26. Pharciceras involutum n. sp. from Hassi Nebech, holotype A

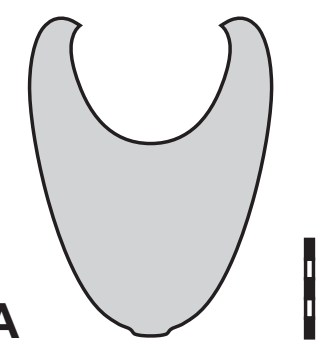
MB.C.22113; A. Cross-section, $\times 2$; B. Suture at $16.5 \mathrm{~mm}$ wh, $\times 4$.

\section{Extropharciceras Bockwinkel, Becker \& Ebbighausen, 2009}

Type species. Extropharciceras conex Bockwinkel et al., 2009.

For a detailed discussion of the genus see Bockwinkel et al. (2009). 


\section{Extropharciceras arenicum (Petter, 1959)}

Figures 27A-D, F-H, J-L, 28A-C, cf. 27E, I, 28D

Lectotype. The original specimen figured by Petter (1959, pl. 9, figs 3, 3a), selected by Bockwinkel et al. (2009).

Material. More than 30 specimens up to $35 \mathrm{~mm}$ conch diameter, including MB.C.22114.1-MB.C.22114.13, and four specimens identified as cf. arenicum (MB.C.22115.1-MB.C.22115.2 and MB.C.22139.1-MB.C.22139.2).

Diagnosis (emend.). Medium-sized; first two whorls until $4 \mathrm{~mm} \mathrm{dm}$ thinly pachyconic, with nearly constant whorl $(\mathrm{ww} / \mathrm{dm}=\mathrm{ca}$. 0.65$)$ and umbilical width (uw $/ \mathrm{dm}=0.35-0.38$ ) and falling WER ratios (down to 1.60); intermediate stages (3rd to 6th whorl) until ca. $20 \mathrm{~mm} \mathrm{dm}$ gradually weakly depressed and subinvolute (uw/dm rates down to ca. 0.20 ) with rapidly rising WER up to 2.00; mature whorls slightly com-
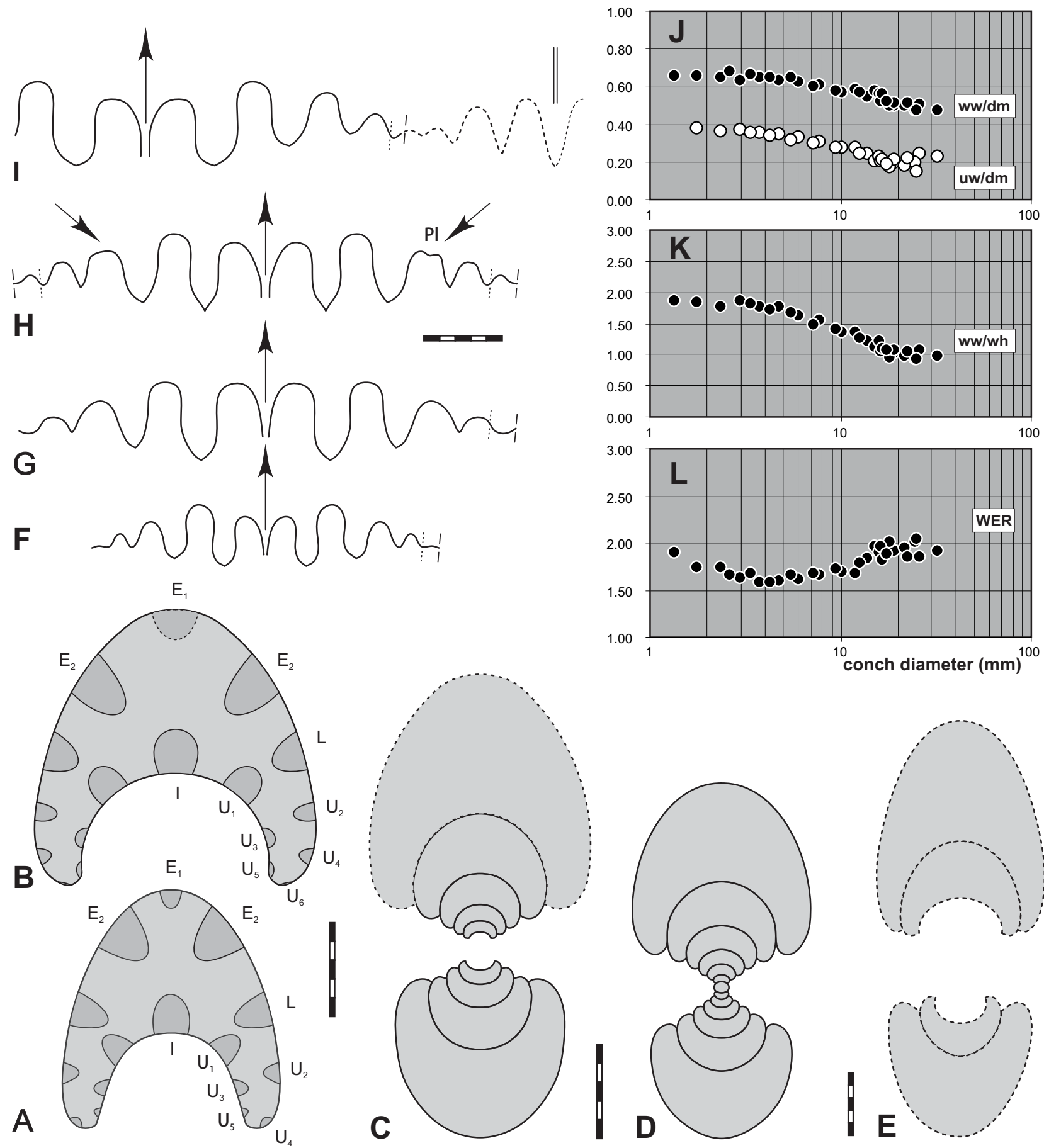

Figure 27. Extropharciceras arenicum (Petter, 1959) from Hassi Nebech, including calcareous cf. specimens; A. Septal face of MB.C.22114.1, $\times 3$; B. Septal face of MB.C.22114.2, $\times 3$; C. Cross-section of MB.C.22114.3, $\times 3$; D. Cross-section of MB.C.22114.4, $\times$ 4; E. Reconstructed cross-section of cf. specimen MB.C.22139.2, $\times 2$; F. Suture of MB.C.22114.5 at $7.5 \mathrm{~mm}$ wh, $\times 2.5$; G. Suture of MB.C.22114.1 at $12 \mathrm{~mm}$ wh, $\times 2.5$; H. Suture of MB.C.22114.6 at $12.3 \mathrm{~mm}$ wh, $\times 2.5$; I. Suture of cf. specimen MB.C.22139.1 (partly reconstructed) at $8.5 \mathrm{~mm}$ wh, $\times 2.5 ; \mathbf{J}-\mathbf{L}$. Ontogenetic development of ww/dm, uw/dm, ww/wh, and WER. 


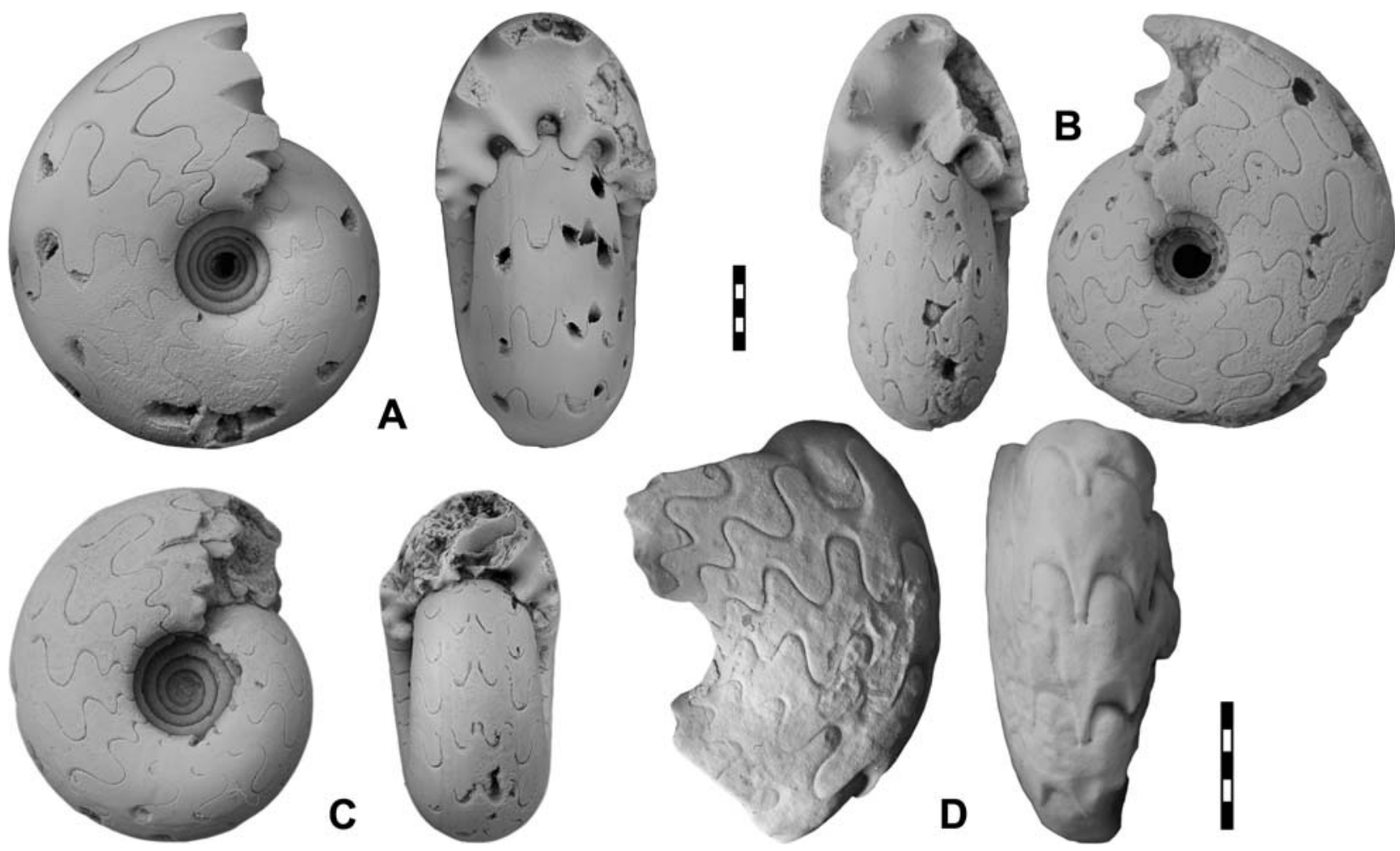

Figure 28. Extropharciceras arenicum (Petter, 1959), including a cf. specimen, from Hassi Nebech; A. MB.C.22114.6, $\times 2$ (with well developed plurilobes); B. MB.C.22114.1, $\times 2$; C. MB.C.22114.7, $\times 3$; D. cf. specimen MB.C.22139.1, $\times 2$.

pressed (ww/wh down to 0.80 ) with gently rounded flanks converging to a well-rounded venter; WER, ww/wh and uw/dm values stagnate. Growth lines strongly biconvex, with high ventrolateral salient lying only in slightly ribbed early stages in weak spiral furrows. Mature sutures with moderately short, divergent $\mathrm{E}_{1}$ lobe, high and narrow median saddle, deep, lanceolate $\mathrm{E}_{2}$ lobe, very high, narrow $\mathrm{E}_{2} \mathrm{~L}$ saddle, diverging to lanceolate $\mathrm{L}$ lobe, not as deep as the $\mathrm{E}_{2}$, moderately high and wide dorsolateral saddle, asymmetrically pointed $\mathrm{U}_{2}$ lobe, two descending saddles between the small rounded, $U$ lobes, three rounded internal $U$ lobes, and deep, pointed I lobe. Suture formula: $\left(E_{2} E_{1} E_{2}\right) L_{2} U_{4} U_{6}: U_{5} U_{3} U_{1} I$.

Table 25. Conch ontogeny (Figs 27A-D, J-L) of Extropharciceras arenicum (Petter, 1959) from Hassi Nebech.

\begin{tabular}{llll}
\hline $\mathrm{dm}$ & conch shape & whorl cross-section shape & whorl expansion \\
\hline $2 \mathrm{~mm}$ & thinly pachyconic; subevolute & moderately depressed; strongly embracing & moderate \\
& $($ ww/dm $\sim 0.65 ;$ uw/dm $\sim 0.35)$ & $($ ww/wh $\sim 1.80 ;$ IZR $\sim 0.33)$ & $($ WER $\sim 1.75)$ \\
$8 \mathrm{~mm}$ & thinly pachyconic; subevolute & moderately depressed; strongly embracing & low \\
& $($ ww/dm $\sim 0.60 ;$ uw/dm $\sim 0.30)$ & $($ ww/wh $\sim 1.50 ;$ IZR $\sim 0.43)$ & $($ WER $\sim 1.70)$ \\
$20 \mathrm{~mm}$ & thickly discoidal; subinvolute & weakly depressed; very strongly embracing & moderate \\
& $($ ww/dm $\sim 0.50 ;$ uw/dm $\sim 0.20)$ & $($ ww/wh $\sim 1.00 ;$ IZR $\sim 0.45)$ & $($ WER $\sim 2.00)$ \\
$35 \mathrm{~mm}$ & thinly discoidal; subinvolute & weakly compressed; very strongly embracing & moderate \\
& $($ ww/dm $\sim 0.40 ;$ uw/dm $\sim 0.20)$ & $($ ww/wh $\sim 0.80 ;$ IZR $\sim 0.45)$ & $($ WER $\sim 2.00)$
\end{tabular}

Table 26. Suture formula (Figs 27A, B, F-H), conch characteristics, and ornament of Extropharciceras arenicum from Hassi Nebech.

$\left(\mathrm{E}_{2} \mathrm{E}_{1} \mathrm{E}_{2}\right) L U_{2} \mathrm{U}_{4}: \mathrm{U}_{3} \mathrm{U}_{1} \mathrm{l}$ at ca. $10 \mathrm{~mm}$ dm (MB.C.22114.10)

$\left(E_{2} E_{1} E_{2}\right) L U_{2} U_{4}: U_{5} U_{3} U_{1} l$ at ca. $16 \mathrm{~mm}$ dm (MB.C.22114.10)

$\left(E_{2} E_{1} E_{2}\right) L_{2} U_{4} U_{6}: U_{5} U_{3} U_{1}$ l at ca. 25-30 mm dm (MB.C.22114.6, MB.C.22114.2)

$E_{1}$ lobe narrow, deep, funnel-shaped; sometimes asymmetrical plurilobation of the dorsolateral saddle (MB.C.22114.4); $U_{2}$ lobe partly hookshaped; $U_{4}$ lobe small, rounded

venter rounded, umbilicus moderately narrow

early stages with weak ventrolateral furrows or double furrows, weak or absent ribs, no mould constrictions

growth lines strongly biconvex, with high ventrolateral projection 


\section{Extropharciceras applanatum Bensaïd, 1974}

Figures 29-31

Holotype. STIPB-Bensaïd-36 (old: GPI Bo 36), original figured by Bensaïd (1974, pl. 4, figs 7, 7a), re-figured in Figure 30A.

Type locality and horizon. Hassi Nebech, Section 2, SE Tafilalt, main collecting level, probably Taouzites taouzensis Zone (late Givetian).

Material. The holotype, paratype STIPB-Bensaï-39 (old: GPI Bo 39), ca. 90 topotypes up to $50 \mathrm{~mm}$ conch diameter, including MB.C.22116.1-MB.C.22116.14, and five specimens identified as cf. applanatum (MB.C.22117.1-MB.C.22117.5).

Diagnosis (emend). Intermediate-sized, with triphasic post-embryonic conch ontogeny; first two whorls widely umbilicate with increasing umbilical and decreasing whorl widths rate; between 2 and $7 \mathrm{~mm} \mathrm{dm}$, with nearly constant ww/dm around 0.6 but increasingly depressed (ww/wh values up to $2.20-2.40$ ), evolute (uw/dm up to 0.55 ) and slowly expanding (WER down to 1.50); intermediate whorls until ca.

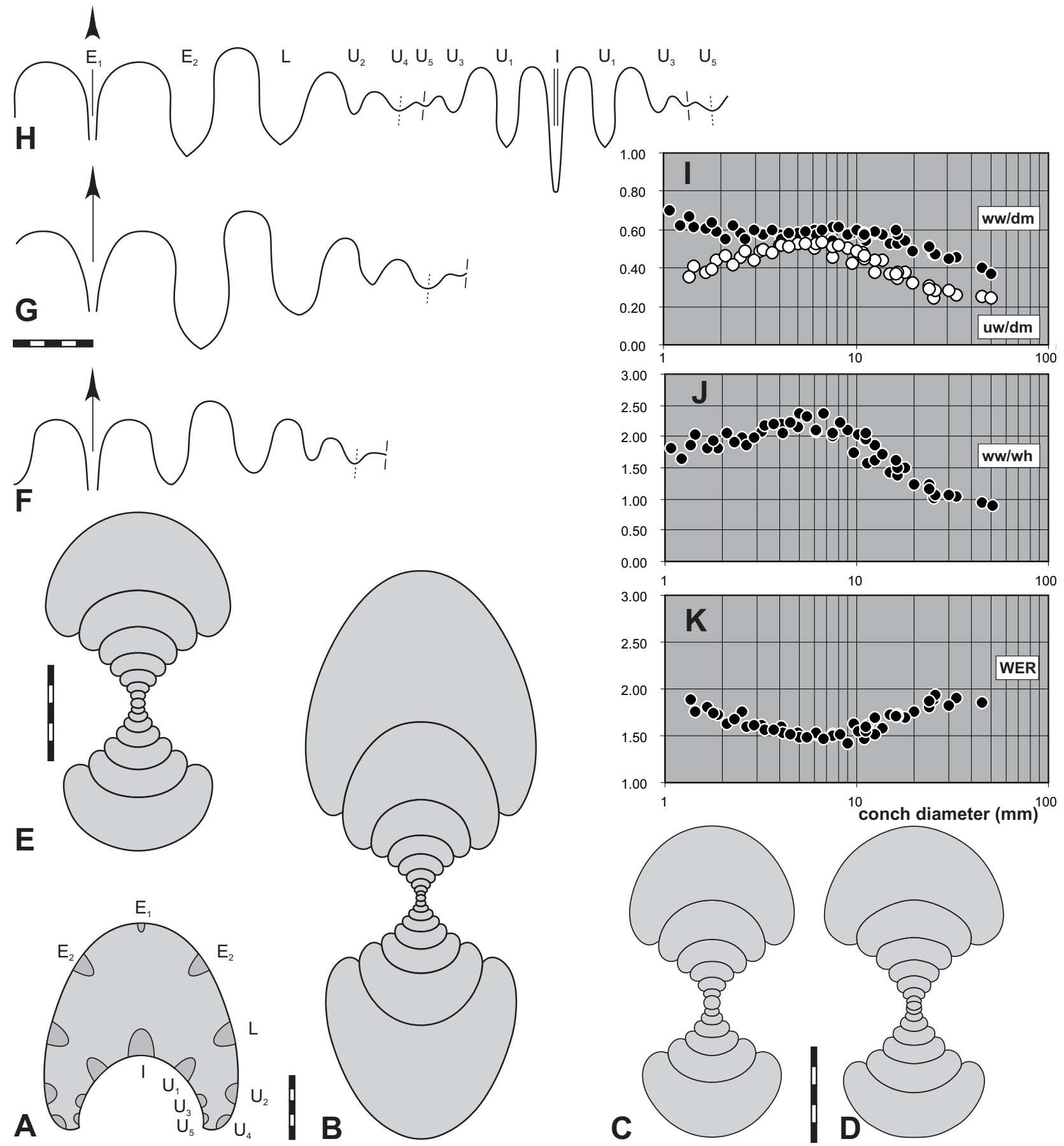

Figure 29. Extropharciceras applanatum (Bensaïd, 1974) Morphotype I (topotypes) from Hassi Nebech; A. Septal face of MB.C.22116.1, $\times 2$; B-E. Cross-sections; B. MB.C.22116.2, $\times 2$; C. MB.C.22116.3, $\times 3$; D. MB.C.22116.4, $\times 3$; E. MB.C.22116.2, inner whorls, $\times 3$; F-H. Sutures. all $\times 2.5$; F. MB.C.22116.5 at $13.5 \mathrm{~mm}$ wh; G. MB.C.22116.2 at $19 \mathrm{~mm}$ wh; H. MB.C.22116.1 at $16.1 \mathrm{~mm}$ wh; I-K. Ontogenetic development of ww/dm, uw/dm, ww/wh, and WER. 


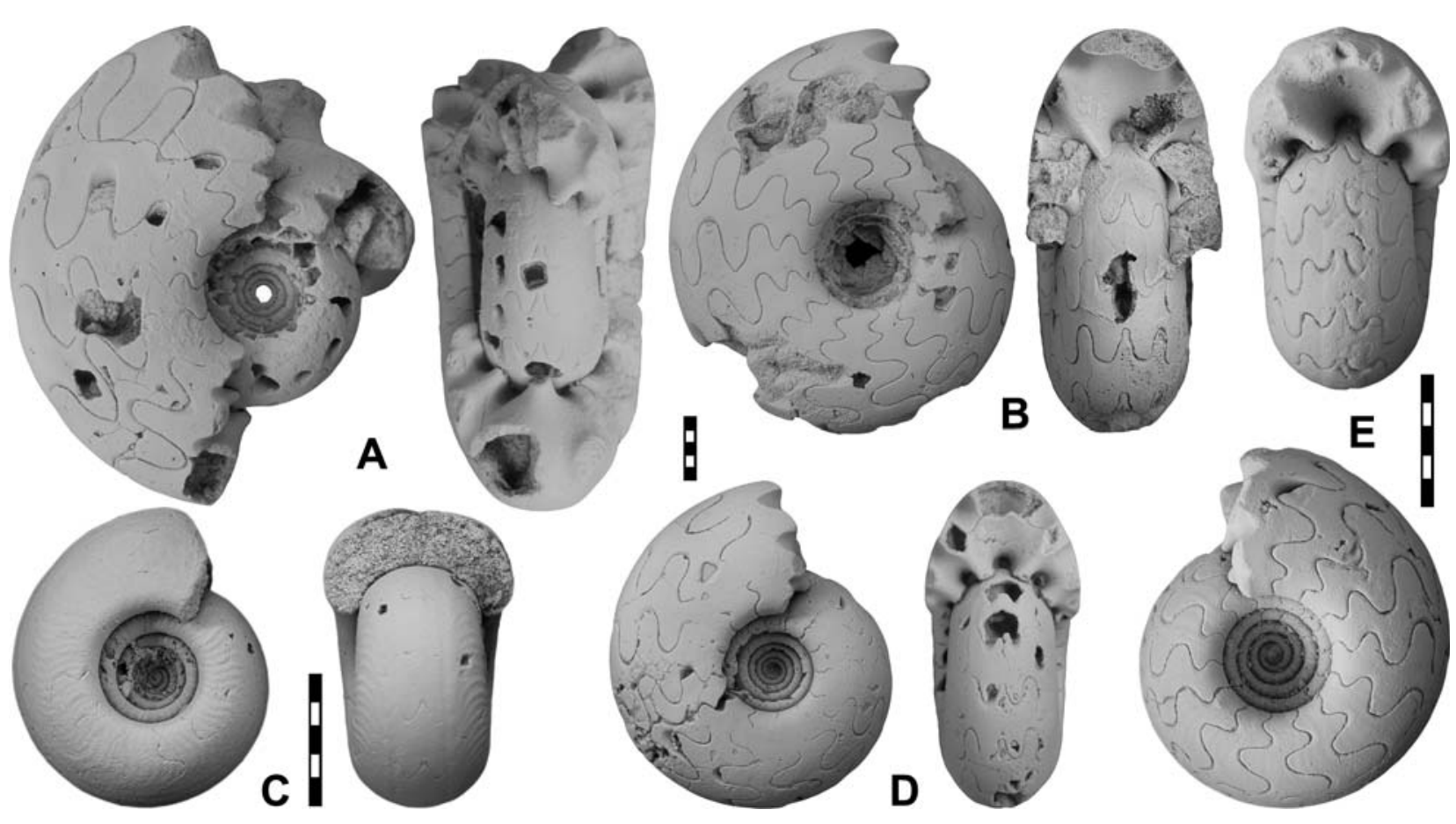

Figure 30. Extropharciceras applanatum (Bensaï, 1974) Morphotype I from Hassi Nebech; A. Holotype STIPB-Bensaïd-36 (old: GPI Bo 36), $\times 1.5$; B. Topotype MB.C.22116.5, $\times 1.5$; C. Topotype MB.C.22116.6, $\times 3$ (relatively involute juvenile specimen); D. Topotype MB.C.22116.7, $\times 1.5$; E. Topotype MB.C.22116.8, $\times 3$.
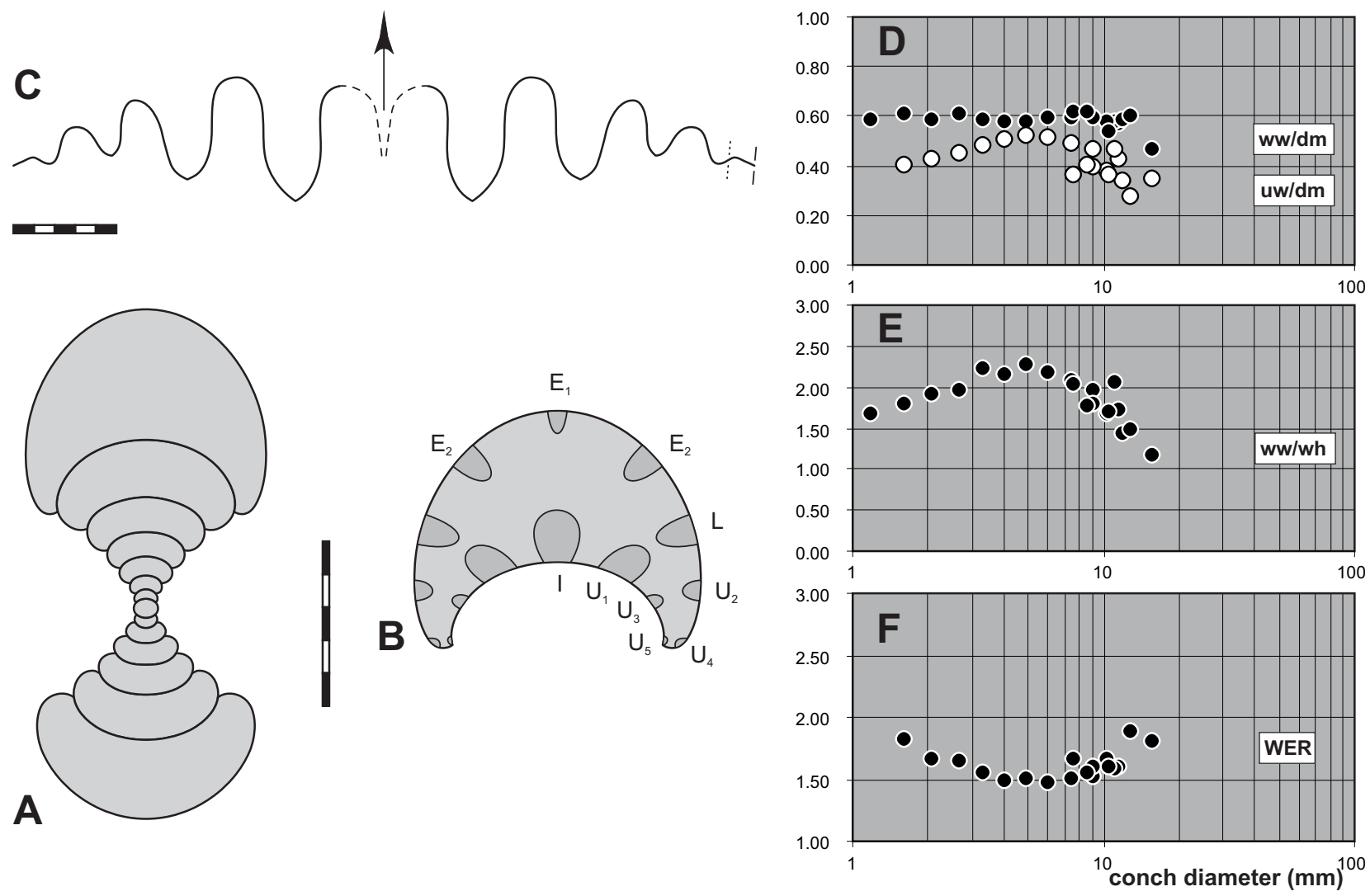

Figure 31. Extropharciceras applanatum (Bensaid, 1974) Morphotype II and a cf. specimen from Hassi Nebech; A. Cross-section of MB.C.22116.14, ×4; B. Septal face of relatively involute cf. specimen MB.C.22117.1, × 4; C. Suture of MB.C.22116.15 at ca. $14 \mathrm{~mm}$ wh, $\times 2.5$; D-F. Ontogenetic development of ww/dm, uw/dm, ww/wh, and WER of all available specimens of Morphotype II. 
Table 27. Conch ontogeny (Figs 29A-E, I-K) of Extropharciceras applanatum (Bensaïd, 1974) morphotype I

\begin{tabular}{|c|c|c|c|}
\hline $\mathrm{dm}$ & conch shape & whorl cross-section shape & whorl expansion \\
\hline $2 \mathrm{~mm}$ & $\begin{array}{l}\text { thickly discoidal; subevolute } \\
\text { (ww/dm =0.50-0.60; uw/dm } \sim 0.45 \text { ) }\end{array}$ & $\begin{array}{l}\text { moderately depressed; moderately embracing } \\
(\mathrm{w} w / \mathrm{wh}=1.90-2.00 ; \mathrm{IZR} \sim 0.25)\end{array}$ & $\begin{array}{l}\text { low } \\
\text { (WER } \sim 1.70)\end{array}$ \\
\hline $5 \mathrm{~mm}$ & $\begin{array}{l}\text { thickly discoidal; evolute } \\
(\mathrm{ww} / \mathrm{dm}=0.50-0.60 ; \mathrm{uw} / \mathrm{dm}=0.50-0.55)\end{array}$ & $\begin{array}{l}\text { strongly depressed; moderately embracing } \\
(\mathrm{ww} / \mathrm{wh}=2.20-2.40 ; \mathrm{IZR}=0.25-0.30)\end{array}$ & $\begin{array}{l}\text { low } \\
\text { (WER } \sim 1.50)\end{array}$ \\
\hline $10 \mathrm{~mm}$ & $\begin{array}{l}\text { thickly discoidal; subevolute to evolute } \\
(\mathrm{ww} / \mathrm{dm}=0.50-0.60 ; \mathrm{uw} / \mathrm{dm}=0.40-0.50)\end{array}$ & $\begin{array}{l}\text { moderately depressed; strongly embracing } \\
(\mathrm{w} w / \mathrm{wh}=1.70-2.00 ; \mathrm{IZR}=0.30-0.40)\end{array}$ & $\begin{array}{l}\text { low } \\
(\text { WER }=1.50-1.60)\end{array}$ \\
\hline $25 \mathrm{~mm}$ & $\begin{array}{l}\text { thinly discoidal; subinvolute } \\
\text { (ww/dm =0.45-0.50; uw/dm } \sim 0.25 \text { ) }\end{array}$ & $\begin{array}{l}\text { weakly depressed; strongly embracing } \\
(\mathrm{w} w / \mathrm{wh}=1.00-1.20 ; \mathrm{IZR}=0.35-0.40)\end{array}$ & $\begin{array}{l}\text { moderate } \\
(\mathrm{WER}=1.80-1.95)\end{array}$ \\
\hline $45 \mathrm{~mm}$ & $\begin{array}{l}\text { thinly discoidal; subinvolute } \\
\text { (ww/dm } \sim 0.40 \text { uw/dm } \sim 0.25 \text { ) }\end{array}$ & $\begin{array}{l}\text { weakly compressed; strongly embracing } \\
\text { (ww/wh } \sim 0.90 ; \mathrm{IZR}=0.35-0.40 \text { ) }\end{array}$ & $\begin{array}{l}\text { moderate } \\
(\text { WER } \sim 1.85)\end{array}$ \\
\hline
\end{tabular}

Table 28. Conch ontogeny (Figs 31A, B, D-F) of Extropharciceras applanatum (Bensaïd, 1974) morphotype II

\begin{tabular}{|c|c|c|c|}
\hline $\mathrm{dm}$ & conch shape & whorl cross-section shape & whorl expansion \\
\hline $2 \mathrm{~mm}$ & $\begin{array}{l}\text { thickly discoidal; subevolute } \\
\text { (ww/dm } \sim 0.60 ; \text { uw/dm } \sim 0.43 \text { ) }\end{array}$ & $\begin{array}{l}\text { moderately depressed; strongly embracing } \\
\text { (ww/wh } \sim 1.90 \text {; IZR } \sim 0.35 \text { ) }\end{array}$ & $\begin{array}{l}\text { low } \\
\text { (WER } \sim 1.65)\end{array}$ \\
\hline $5 \mathrm{~mm}$ & $\begin{array}{l}\text { thickly discoidal; evolute } \\
\text { (ww/dm 0.60; uw/dm 0.52) }\end{array}$ & $\begin{array}{l}\text { strongly depressed; moderately embracing } \\
\text { (ww/wh } \sim 2.25 ; \text { IZR } \sim 0.30 \text { ) }\end{array}$ & $\begin{array}{l}\text { low } \\
(\text { WER } \sim 1.50)\end{array}$ \\
\hline $10 \mathrm{~mm}$ & $\begin{array}{l}\text { thickly discoidal; subevolute to evolute } \\
\text { (ww/dm } \sim 0.60 ; \text { uw/dm } \sim 0.45)\end{array}$ & $\begin{array}{l}\text { moderately depressed; strongly embracing } \\
\text { (ww/wh } \sim 1.75 ; \text { IZR } \sim 0.36 \text { ) }\end{array}$ & $\begin{array}{l}\text { low } \\
(\text { WER } \sim 1.60)\end{array}$ \\
\hline $15 \mathrm{~mm}$ & $\begin{array}{l}\text { thickly discoidal; subevolute } \\
\text { (ww/dm } \sim 0.50 ; \mathrm{uw} / \mathrm{dm} \sim 0.35)\end{array}$ & $\begin{array}{l}\text { weakly depressed; strongly embracing } \\
\text { (ww/wh } \sim 1.20 ; \text { IZR } \sim 0.36)\end{array}$ & $\begin{array}{l}\text { moderate } \\
(\text { WER } \sim 1.80)\end{array}$ \\
\hline
\end{tabular}

Table 29. Suture formula (Figs 29A, F-H, 31B, C), conch characteristics, and ornament of Extropharciceras applanatum (Bensaïd, 1974)

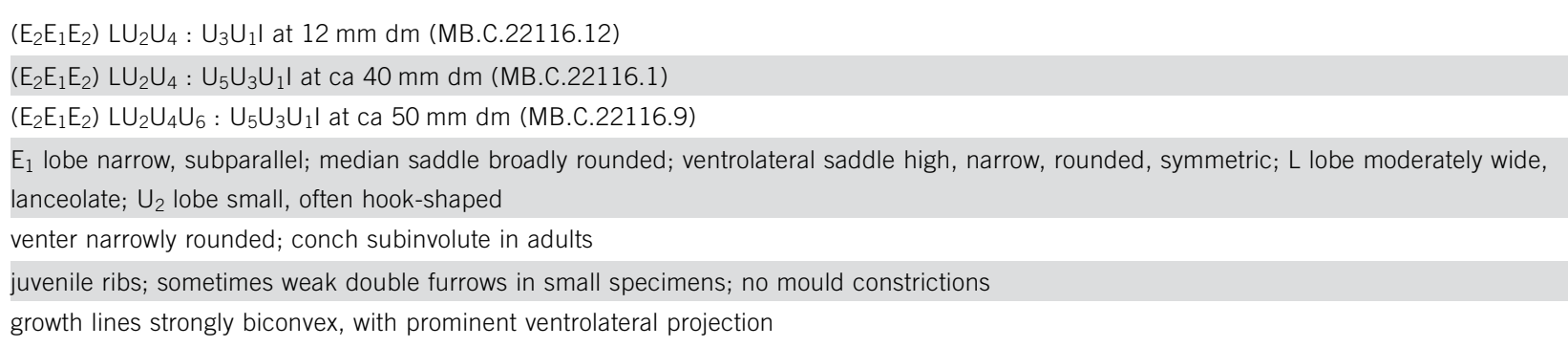

$30 \mathrm{~mm} \mathrm{dm}$ gradually or abruptly less depressed and subinvolute (mature uw/dm $\sim 0.25$ ), with sharply rising WER to values slightly below 2.00; adult whorls just compressed (ww/wh ca. 0.90), with rounded to slightly flattened venter, constant uw/dm and WER values. Postembryonic whorls until $12 \mathrm{~mm} \mathrm{dm}$ with delicate, dense, falcate, subumbilical ribbing; growth lines strongly biconvex, with narrow and high ventrolateral furrows sitting in weak double furrows that disappear in median stages. Sutures from 30-35 mm dm with narrow, moderately deep, funnel-shaped $\mathrm{E}_{1}$ lobe, relatively wide, high median saddle, deep, lanceolate $\mathrm{E}_{2}$ lobe, high, very narrow ventrolateral saddle, deeply rounded to lanceolate $\mathrm{L}$ lobe, not as deep as the $E_{2}$, descending height of saddles adjacent to the small, pointed $U_{2}$ lobe, rounded $U_{4}$ lobe, and very shallow $\mathrm{U}_{6}$ lobe; internal $\mathrm{U}_{5}$ and $\mathrm{U}_{3}$ lobes small, $\mathrm{U}_{1}$ lobe narrow and lanceolate, I lobe very deep and narrow. Suture formula: $\left(\mathrm{E}_{2} \mathrm{E}_{1} \mathrm{E}_{2}\right) \mathrm{LU}_{2} \mathrm{U}_{4} \mathrm{U}_{6}: \mathrm{U}_{5} \mathrm{U}_{3} \mathrm{U}_{1} \mathrm{I}$. 


\section{Extropharciceras n. sp. 2}

Figure 32

Material. Only MB.C.22118.
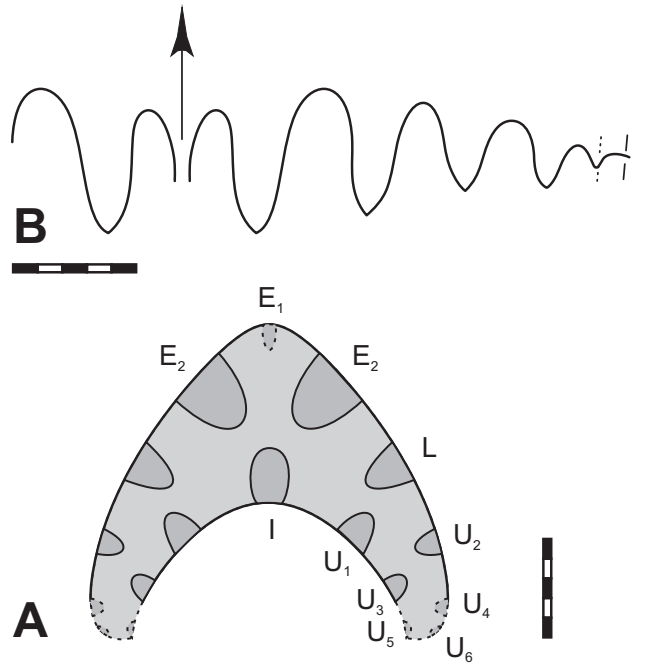

Figure 32. Extropharciceras n. sp. 2 from Hassi Nebech, MB.C.22118; A. Partly reconstructed septal face, $\times 2$; B. Suture at ca. $13 \mathrm{~mm}$ wh, $\times 2.5$.

\section{Lunupharciceras Korn \& Klug, 2002}

Type species. Goniatites lunulicosta Sandberger \& Sandberger, 1850.

\section{Lunupharciceras incisum n. sp.}

Figures 33, 34

Derivation of name. After the narrow, deeply incised flank lobes.

Holotype. MB.C.22119.1 (Figs 33A and 34C), which is fully septate and shows the characteristic inner and outer sutures and conch shape.

Type locality and horizon. Hassi Nebech, Section 2, SE Tafilalt, Bed 11a and main collecting interval, probably Taouzites taouzensis Zone (late Givetian). Material. Ten specimens up to ca. $25 \mathrm{~mm}$ conch diameter, including MB.C.22119.1-MB.C.22119.9.

Diagnosis. Slightly depressed and evolute conch shape relatively uniform throughout post-embryonic ontogeny; umbilical width near $50 \% \mathrm{dm}$, with slightly and gradually falling relative whorl width (ww/dm from ca. 0.40 at $5 \mathrm{~mm}$ dm down to ca. 0.35 at $20 \mathrm{~mm} \mathrm{dm}$, ww/wh from ca. 1.40 down to 1.10 at same sizes); WER expanding slowly (from 1.80 down to 1.60); mature stages unknown. No ribbing or ventrolateral spiral furrows; growth ornament unknown. Sutures from ca. $16 \mathrm{~mm} \mathrm{dm}$ with shortened, narrow divergent $\mathrm{E}_{1}$ lobe, moderately high median saddle, very deep, narrow, lanceolate $\mathrm{E}_{2}$ lobe, high and narrow, constricted ventrolateral and dorsolateral saddles, deep, narrow, lanceolate $\mathrm{L}$ lobe, ascending three outer $U$ lobes, the first two narrow and lanceolate, the third asymmetrical, subacute, a low saddle at the umbilical seam, a small internal $\mathrm{U}$ lobe, a deep, lanceolate $\mathrm{U}_{1}$ lobe, and a very deep, pointed I lobe. Suture formula $\left(\mathrm{E}_{2} \mathrm{E}_{1} \mathrm{E}_{2}\right) \mathrm{LU}_{2} \mathrm{U}_{3} \mathrm{U}_{4}: \mathrm{U}_{5} \mathrm{U}_{1} \mathrm{I}$ or $\left(\mathrm{E}_{2} \mathrm{E}_{1} \mathrm{E}_{2}\right) \mathrm{LU}_{2} \mathrm{U}_{3} \mathrm{U}_{4 \mathrm{e}}: \mathrm{U}_{4 \mathrm{i}} \mathrm{U}_{1} \mathrm{I}$.

Table 30. Conch ontogeny (Figs 33A-D, J-L) of Lunupharciceras incisum n. sp.

\begin{tabular}{|c|c|c|c|}
\hline$d m$ & conch shape & whorl cross-section shape & whorl expansion \\
\hline $2 \mathrm{~mm}$ & $\begin{array}{l}\text { thinly discoidal; subevolute } \\
\text { (ww/dm } \sim 0.45 ; \text { uw/dm } \sim 0.40 \text { ) }\end{array}$ & $\begin{array}{l}\text { weakly depressed; weakly embracing } \\
\text { (ww/wh } \sim 1.30 ; \text { IZR } \sim 0.15 \text { ) }\end{array}$ & $\begin{array}{l}\text { moderate } \\
(\text { WER } \sim 1.95)\end{array}$ \\
\hline $8 \mathrm{~mm}$ & $\begin{array}{l}\text { thinly discoidal; evolute } \\
\text { (ww/dm } \sim 0.40 ; \text { uw/dm } \sim 0.50 \text { ) }\end{array}$ & $\begin{array}{l}\text { weakly depressed; moderately embracing } \\
\text { (ww/wh } \sim 1.40 ; \text { IZR } \sim 0.20 \text { ) }\end{array}$ & $\begin{array}{l}\text { low } \\
(\text { WER } \sim 1.70)\end{array}$ \\
\hline $18 \mathrm{~mm}$ & $\begin{array}{l}\text { thinly discoidal; evolute } \\
\text { (ww/dm } \sim 0.35 ; \text { uw/dm } \sim 0.50 \text { ) }\end{array}$ & $\begin{array}{l}\text { weakly depressed; moderately embracing } \\
\text { (ww/wh }=1.15-1.30 ; \text { IZR } \sim 0.25 \text { ) }\end{array}$ & $\begin{array}{l}\text { low } \\
(\text { WER } \sim 1.65)\end{array}$ \\
\hline
\end{tabular}

Table 31. Suture formula (Figs 33A, E-I), conch characteristics, and ornament of Lunupharciceras incisum n. sp.

$\left(E_{2} E_{1} E_{2}\right) L U_{2} U_{3}: U_{4}: U_{1} l$ at $10 \mathrm{~mm} d m(M B . C .22119 .5)$

$\left(E_{2} E_{1} E_{2}\right) L U_{2} U_{3} U_{4 e}: U_{4 i} U_{1} I$ at $15,18,24 \mathrm{~mm} d m$ (MB.C.22119.1, MB.C.22119.5, MB.C.22119.6)

internally/externally asymmetrical development of the $U$ lobes from $U_{3}$; lateral saddles similarly shaped and narrow

venter broadly rounded, umbilical wall deep

no ribs of early stages; no ventrolateral furrows; no mould constrictions

growth lines not visible 


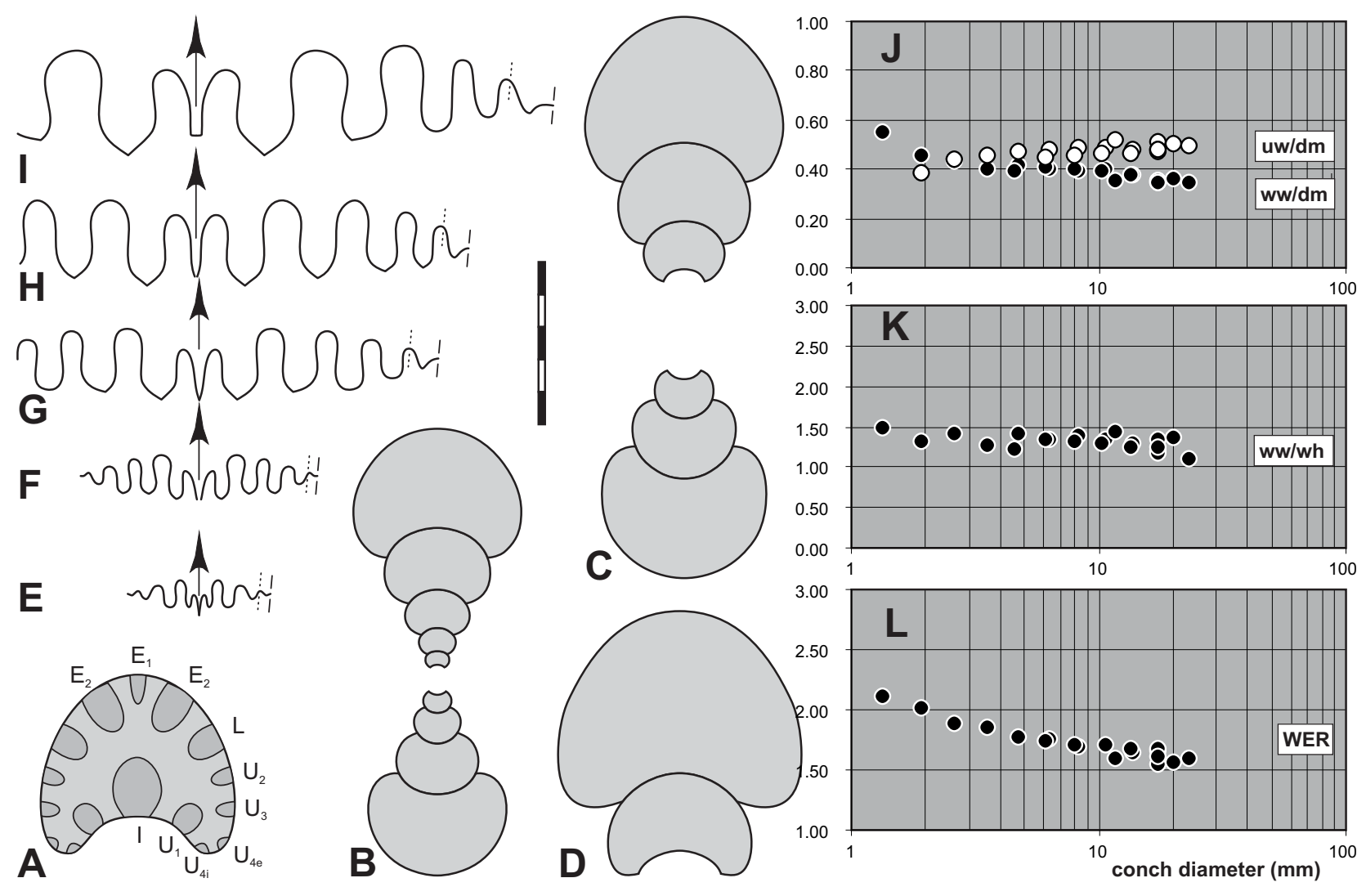

Figure 33. Lunupharciceras incisum n. sp. from Hassi Nebech; A. Septal face of holotype MB.C.22119.1, $\times 4$; B-D. Cross-sections, all $\times$ 4; B. Paratype MB.C.22119.2; C. Paratype MB.C.22119.3; D. Paratype MB.C.22119.4; E-I. Sutures, all $\times 4$; E. Paratype MB.C.22119.5 at $1.9 \mathrm{~mm}$ wh; F. Paratype MB.C.22119.5 at $2.7 \mathrm{~mm}$ wh; G. Paratype MB.C.22119.5 at $5.2 \mathrm{~mm}$ wh; H. Paratype MB.C.22119.4 at $6.5 \mathrm{~mm}$ wh; I. Paratype MB.C.22119.6 at $7.3 \mathrm{~mm}$ wh; J-L. Ontogenetic development of ww/dm, uw/dm, ww/wh, and WER of all available specimens.
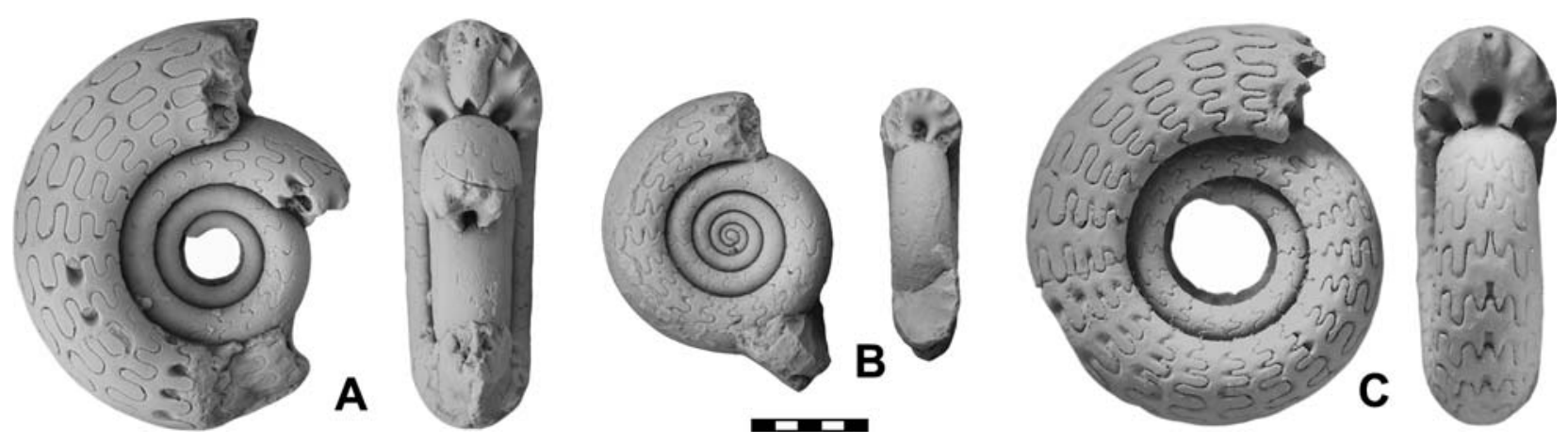

Figure 34. Lunupharciceras incisum n. sp. from Hassi Nebech; A. Paratype MB.C.22119.5; B. Paratype MB.C.22119.7; C. Holotype MB.C.22119.1; all $\times 2.5$.

\section{Lunupharciceras lunulicosta (Sandberger \& Sandberger, 1850)}

Figures 35, 36

Lectotype. The original of Sandberger \& Sandberger (1849, pl. 3, fig. 14a) designated and re-illustrated in House \& Ziegler (1977), part of No. 27 of the Sandberger Collection in the Landesmuseum Wiesbaden.

Type locality and horizon. Oberscheld, Lahn-Dill area, southern Rhenish Massive; Red Ironstone Formation, late Givetian, precise level unknown.

Material. Five incomplete or poorly preserved specimens encrusted by goethite from Hassi Nebech (MB.C.22120.1-MB.C.22120.5) and a topotype from Oberscheld, originally from the collection at Münster (MB.C.22121).

Diagnosis (emend.). Post-embryonic whorls up to ca. $9 \mathrm{~mm} \mathrm{dm}$ smooth, increasingly subevolute (uw/dm up to 0.48 ), depressed, with decreasing relative whorl width (ww/dm falling from ca. 0.50 to 0.30 ) and slightly decreasing ww/wh values while WER values fluctuate; intermediate to mature stages increasingly subinvolute (uw/dm down to ca. $0.30>50 \mathrm{~mm} \mathrm{dm}$ ), with at first weakly depressed and finally compressed, moder- 
ately expanding whorls (WER > 1.90) with flattened flanks, ventrolateral furrows and flattened venter. Growth ornament strongly biconvex with high and narrow ventrolateral projection adjacent to the ventral margins. Mature sutures with divergent, shortened $\mathrm{E}_{1}$ lobe, moderately high median saddle, very deep, lanceolate $\mathrm{E}_{2}$ lobe, very high, narrow, constricted $\mathrm{E}_{2} \mathrm{~L}$ saddle, three descending, narrow, constricted flank saddles separating narrow, ascending, lanceolate $\mathrm{L}$ and $\mathrm{U}$ lobes, an asymmetrically pointed, small $\mathrm{U}_{4}$ lobe, a small saddle at the umbilical seam, a small internal $\mathrm{U}_{4 \mathrm{i}}$ and a deep, lanceolate $\mathrm{U}_{1}$ lobe, and a narrow, very deep I lobe. Suture formula: $\left(\mathrm{E}_{2} \mathrm{E}_{1} \mathrm{E}_{2}\right) \mathrm{LU}_{2} \mathrm{U}_{3} \mathrm{U}_{4 \mathrm{e}}: \mathrm{U}_{4 \mathrm{i}} \mathrm{U}_{1} \mathrm{I}$

Table 32. Conch ontogeny (Figs 35A-E, G-I) of Lunupharciceras lunulicosta (Sandberger \& Sandberger, 1850) from Hassi Nebech.

\begin{tabular}{|c|c|c|c|}
\hline$d m$ & conch shape & whorl cross-section shape & whorl expansion \\
\hline $2 \mathrm{~mm}$ & thickly discoidal; subevolute & moderately depressed; weakly embracing & moderate \\
\hline $8 \mathrm{~mm}$ & $\begin{array}{l}\text { thinly discoidal; subevolute } \\
\text { (ww/dm } \sim 0.40 ; \text { uw/dm } \sim 0.45 \text { ) }\end{array}$ & $\begin{array}{l}\text { weakly depressed; moderately embracing } \\
\text { (ww/wh } \sim 1.30 ; \text { IZR }=0.20-0.25 \text { ) }\end{array}$ & $\begin{array}{l}\text { low } \\
(\text { WER } \sim 1.70)\end{array}$ \\
\hline $15 \mathrm{~mm}$ & $\begin{array}{l}\text { thinly discoidal; subevolute } \\
\text { (ww/dm } \sim 0.35 ; \text { uw/dm } \sim 0.45 \text { ) }\end{array}$ & $\begin{array}{l}\text { weakly depressed; moderately embracing } \\
\text { (ww/wh } \sim 1.15 ; \text { IZR }=0.25-0.30 \text { ) }\end{array}$ & $\begin{array}{l}\text { moderate } \\
(\text { WER } \sim 1.80)\end{array}$ \\
\hline
\end{tabular}

Table 33. Suture formula (Figs 35B, F), conch characteristics, and ornament of Lunupharciceras lunulicosta (Sandberger \& Sandberger, 1850) from Hassi Nebech.

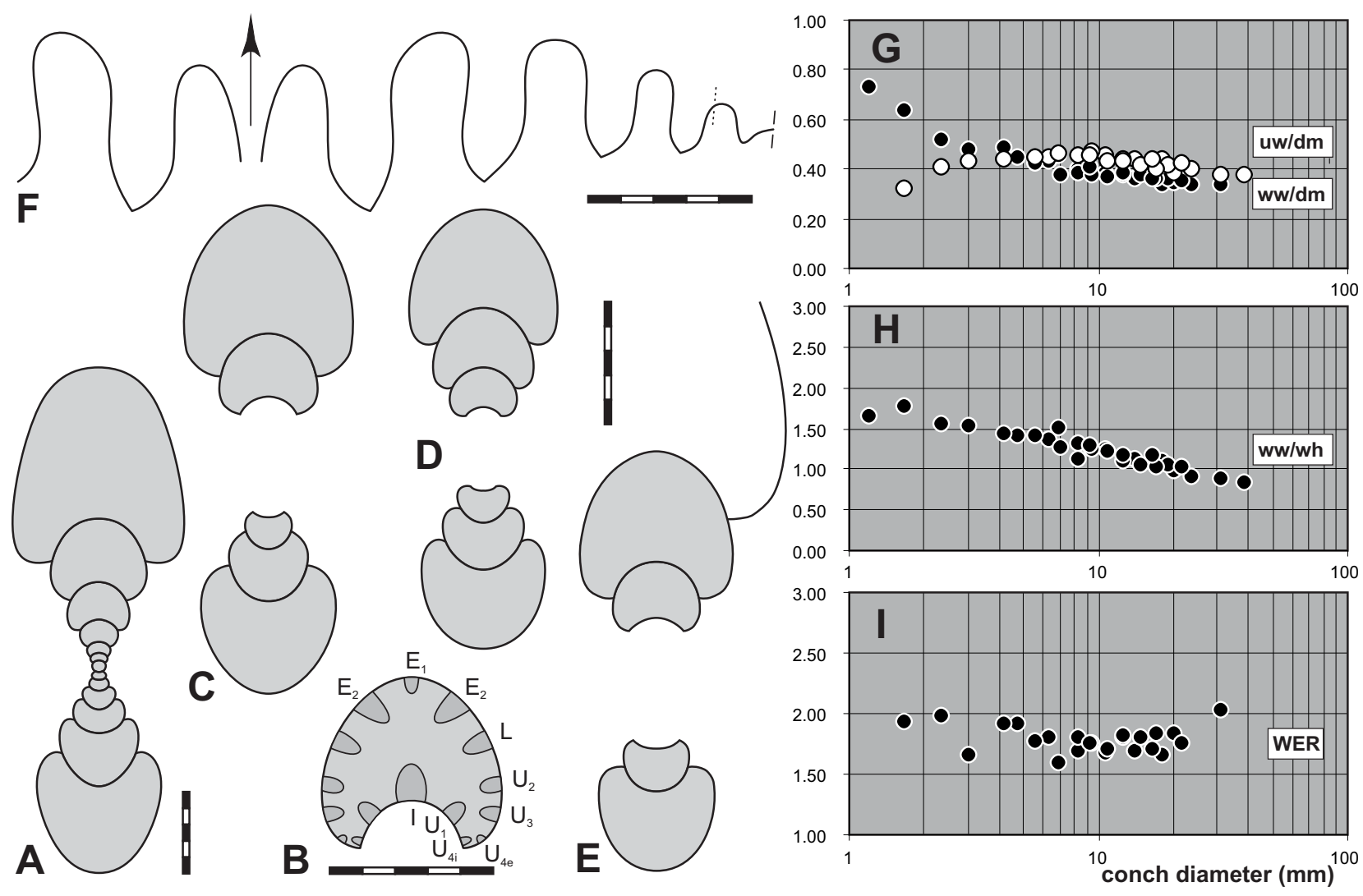

Figure 35. Lunupharciceras lunulicosta (Sandberger \& Sandberger, 1850); A. Cross-section of topotype MB.C.22121 from Sessacker, Oberscheld, $\times 2$; B-F. Specimens from Hassi Nebech; B. Septal face of MB.C.22120.1, $\times 4$; C. Cross-section of MB.C.22120.1, $\times 3$; D. Cross-section of MB.C.22120.2, $\times 3$; E. Cross-section of MB.C.22120.3, $\times 3$; F. Suture of MB.C.22120.3 at ca. $12 \mathrm{~mm}$ wh, $\times 4$; G-I. Ontogenetic development of ww/dm, uw/dm, ww/wh, and WER. 

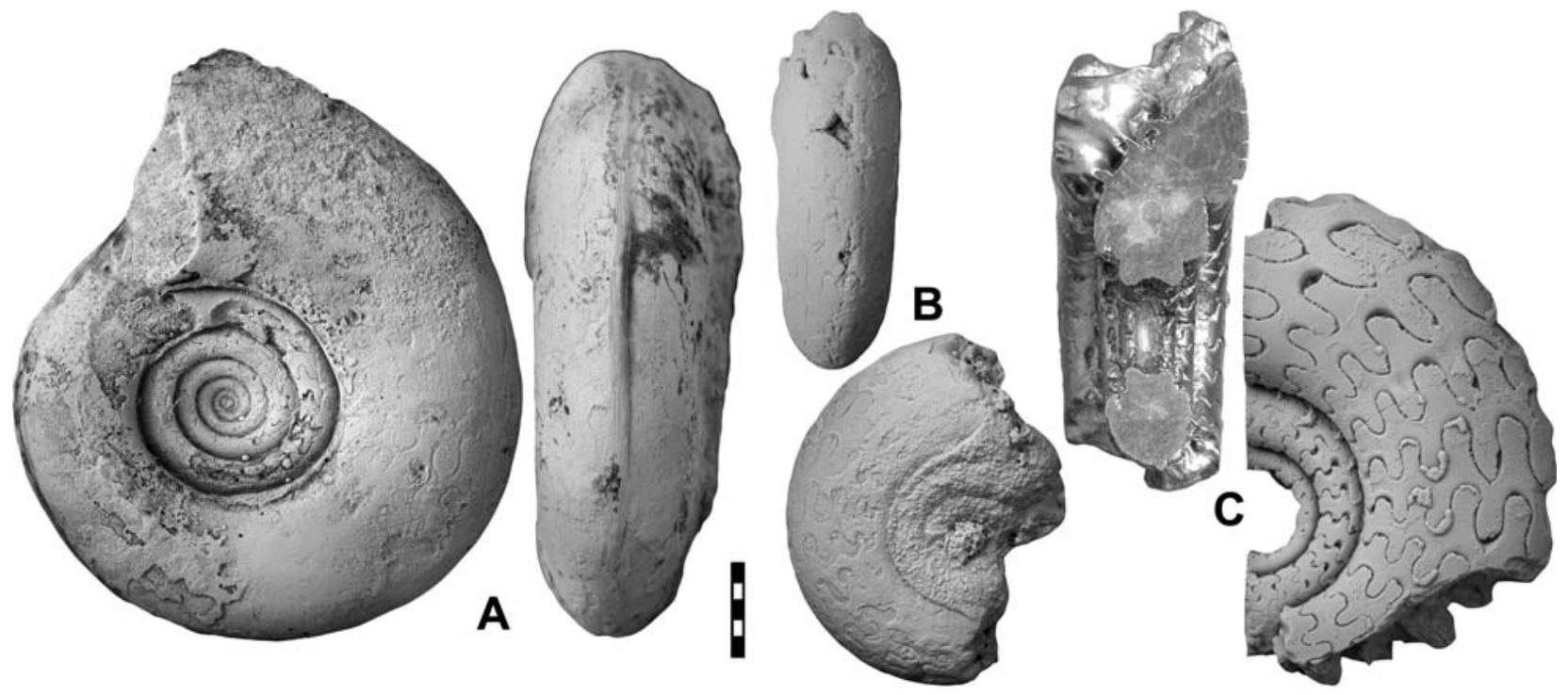

Figure 36. Lunupharciceras lunulicosta (Sandberger \& Sandberger, 1850); A. Topotype MB.C.22121 from Sessacker, Oberscheld (cast), $\times 2$; B. MB.C.22120.4 from Hassi Nebech, $\times 2$; C. MB.C.22120.3 from Hassi Nebech,$\times 2$.

\section{Transpharciceras n. gen.}

Derivation of name. From the Latin trans (= over); due to the transition from Lunupharciceras to species of the new genus with additional $\mathrm{U}$ lobes.

Type species. Transpharciceras procedens $\mathrm{n} . \mathrm{sp}$.

Diagnosis. Early to median stages evolute to subevolute, smooth, with well-rounded flanks and venter, slowly expanding whorls (low WER values near 1.50), decreasing umbilical width and partially lower uw/dm than ww/dm ratios. Mature whorls slightly depressed to compressed, with somewhat higher whorl expansion rates. Mature sutures with shortened, divergent $E_{1}$ lobe, narrow, ascending, similarly-shaped, lanceolate lateral lobes and narrow, characteristically constricted saddles becoming lower towards the umbilicus, including three to four outer $U_{\text {lobes, }}$ with three internal $U$ lobes and narrow, deep I lobe. Suture formulae not yet clearly established, probably $\left(E_{2} E_{1} E_{2}\right) L_{2} U_{3} U_{5}: U_{6} U_{4} U_{1} I$ to $\left(E_{2} E_{1} E_{2}\right)$ $\mathrm{LU}_{2} \mathrm{U}_{3} \mathrm{U}_{5} \mathrm{U}_{7}: \mathrm{U}_{6} \mathrm{U}_{4} \mathrm{U}_{1} \mathrm{I}$.

\section{Transpharciceras procedens $\mathbf{n}$. sp.}

\section{Figures 37,38}

Derivation of name. Due to the progressive addition of a sixth $\mathrm{U}$ lobe in comparison with the five $\mathrm{U}$ lobes in Lunupharciceras.

Holotype. MB.C.22122.1 (Figs 37A, 37F, and 38B).

Type locality and horizon. Hassi Nebech, Section 2, SE Tafilalt, main collecting level, probably Taouzites taouzensis Zone (late Givetian). Material. Four specimens up to $16.5 \mathrm{~mm}$ conch dm (MB.C.22122.1-MB.C.22122.4).

Diagnosis. Early whorls up to ca. $8 \mathrm{~mm} \mathrm{dm} \mathrm{smooth,} \mathrm{moderately} \mathrm{but} \mathrm{increasingly} \mathrm{depressed} \mathrm{(ww/wh} \mathrm{up} \mathrm{to} 1.80$ ), evolute, with broadly rounded venter, slightly decreasing umbilical width ratio (from 0.60 to 0.50$)$, and nearly static, low WER $(1.50-1.60)$; intermediate stages markedly less depressed (ww slightly larger than wh at $15 \mathrm{~mm} \mathrm{dm}$ ), subevolute, with lower uw/dm than ww/dm ratios (ca. 0.40 at $15 \mathrm{~mm} \mathrm{dm}$ ), and slightly higher whorls (WER up to 1.85). No ventrolateral furrows; growth ornament not known. Sutures of intermediate stages with shortened, divergent $E_{1}$ lobe, low median saddle, narrow lanceolate flank lobes ascending towards the umbilicus, descending, very narrow, constricted lateral saddles, three internal $\mathrm{U}$ lobes, and deep, narrow I lobe. Probable suture formula: $\left(\mathrm{E}_{2} \mathrm{E}_{1} \mathrm{E}_{2}\right) \mathrm{LU}_{2} \mathrm{U}_{3} \mathrm{U}_{5}: \mathrm{U}_{6} \mathrm{U}_{4} \mathrm{U}_{1} \mathrm{I}$.

Table 34. Conch ontogeny (Figs 37A-C, G-I) of Transpharciceras procedens n. gen. n. sp.

\begin{tabular}{llll}
\hline $\mathrm{dm}$ & conch shape & whorl cross-section shape & whorl expansion \\
\hline $2 \mathrm{~mm}$ & thinly discoidal; evolute & moderately depressed; weakly embracing & low \\
& $($ ww/dm $\sim 0.35 ;$ uw/dm $\sim 0.60)$ & $($ ww/wh $\sim 1.70 ;$ IZR $\sim 0.15)$ & $($ WER $\sim 1.55)$ \\
$8 \mathrm{~mm}$ & thickly discoidal; evolute & moderately depressed; moderately embracing & low \\
& $($ ww/dm $\sim 0.50 ;$ uw $/ \mathrm{dm} \sim 0.50)$ & $($ ww/wh $\sim 1.80 ;$ IZR $=0.20-0.30)$ & $($ WER $\sim 1.60)$ \\
$13 \mathrm{~mm}$ & thinly discoidal; subevolute & weakly depressed; moderately embracing & low \\
& $($ ww/dm $\sim 0.45 ;$ uw/dm $=0.40-0.45)$ & $($ ww/wh $=1.30-1.40 ;$ IZR $\sim 0.30)$ & $($ WER $\sim 1.70)$ \\
\hline
\end{tabular}


Table 35. Suture formula (Figs 37A, D-F), conch characteristics, and ornament of Transpharciceras procedens $\mathrm{n}$. gen. $\mathrm{n}$. sp.

\section{$\left(E_{2} E_{1} E_{2}\right) L U_{2} U_{3} U_{5}: U_{6} U_{4} U_{1} l$ at $15 \mathrm{~mm}$ dm (MB.C.22122.1)}

asymmetrical development of the $\mathrm{U}$ lobes from $\mathrm{U}_{3}$

venter rounded, umbilical wall steeply rounded

no ribs; no spiral furrows; no mould constrictions

growth lines not visible

$$
\begin{aligned}
& \text { F भी }
\end{aligned}
$$

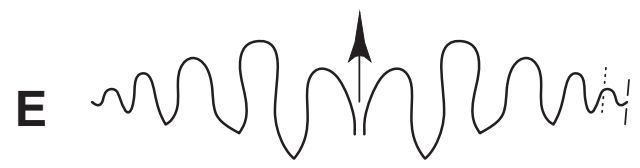

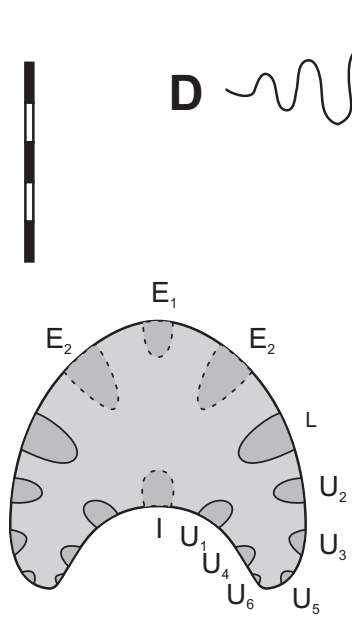

A
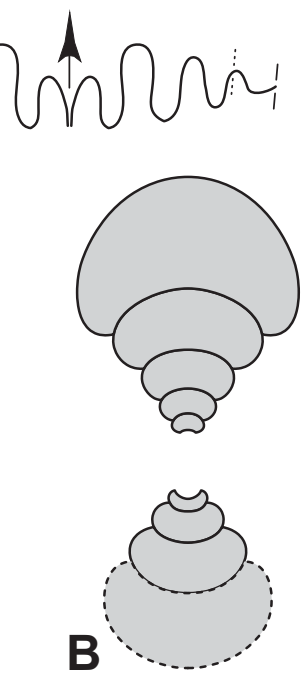
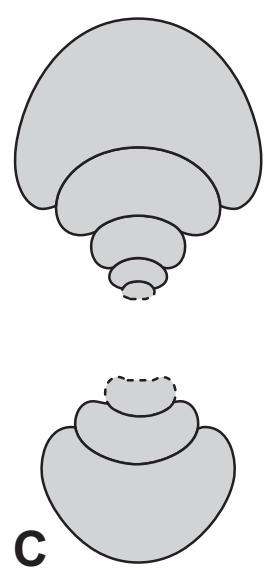

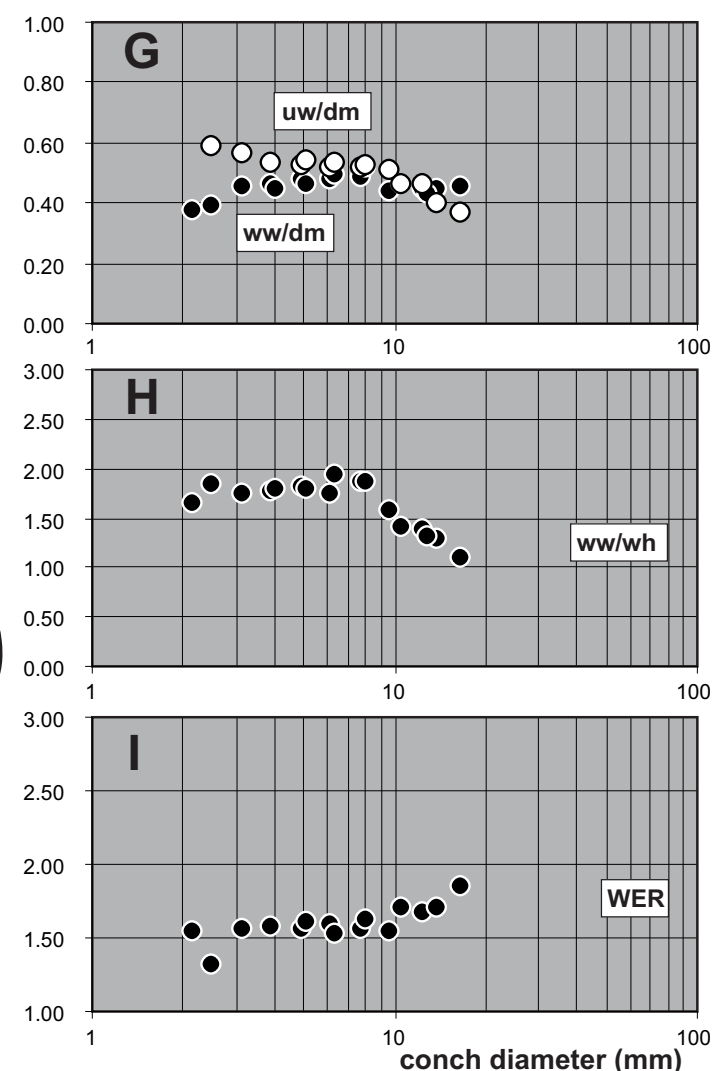

Figure 37. Transpharciceras procedens n. gen. n. sp. from Hassi Nebech; A. Septal face of holotype MB.C.22122.1, $\times 4$; B. Crosssection of paratype MB.C.22122.2, $\times 4$; C. Cross-section of paratype MB.C.22122.3, $\times 4$; D. Suture of paratype MB.C.22122.4 at $1.5 \mathrm{~mm}$ wh, $\times 4$; E. Suture of paratype MB.C. 22122.3 at $1.9 \mathrm{~mm}$ wh, $\times 4$; F. Suture of holotype MB.C.22122.1 at $7 \mathrm{~mm}$ wh, $\times 4$; G-I. Ontogenetic development of ww/dm, uw/dm, ww/wh, and WER of all available specimens.
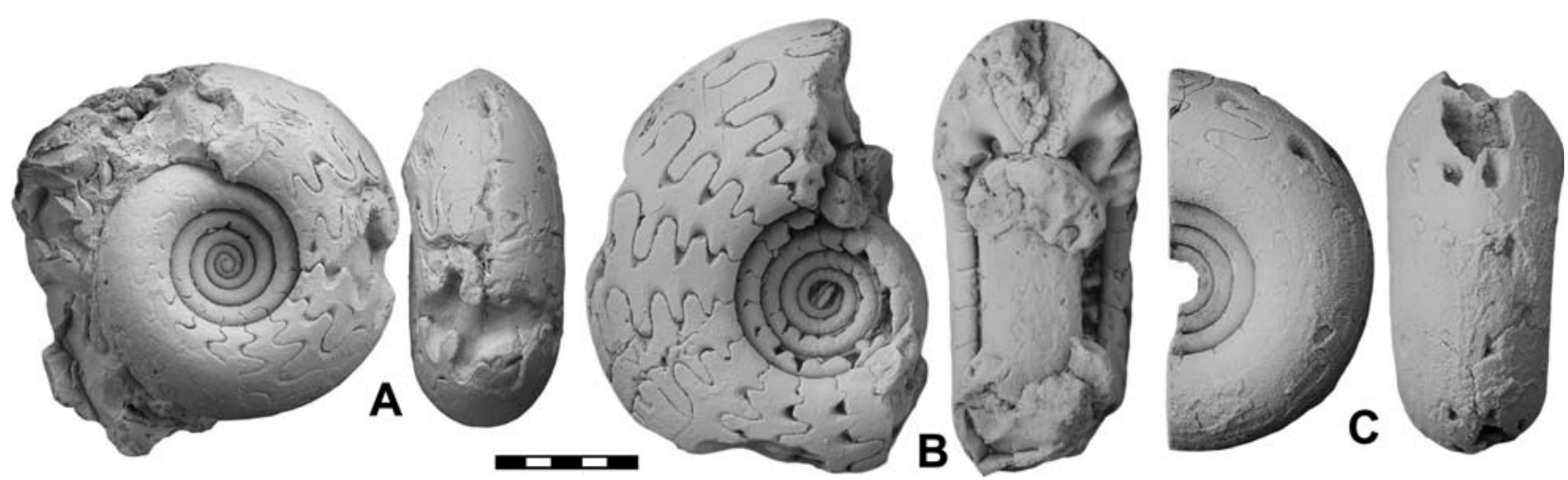

Figure 38. Transpharciceras procedens n. gen. n. sp. from Hassi Nebech; A. Paratype MB.C.22122.4; B. Holotype MB.C.22122.1; C. Paratype MB.C.22122.3; all $\times 3$. 
Subfamily Synpharciceratinae Schindewolf, 1940

\section{Stenopharciceras Montesinos \& Henn, 1986}

Type species. Pharciceras tridens var. kseirense Termier \& Termier, 1950.

\section{Stenopharciceras kseirense (Termier \& Termier, 1950)}

Figures 39, 40

Lectotype. As outlined in Bockwinkel et al. (2009), the lectotype designation in Montesinos \& Henn (1986) is invalid since it was not based on one of the syntypes (Clariond collection) from the Tafilalt. The valid lectotype, designated in Bockwinkel et al. (2009), is the original of Termier \& Termier (1950, pl. 150, fig. 38).

Type locality and horizon. Oued Kseir = Hassi Nebech area, SE Tafilalt, main collecting level, probably Taouzites taouzensis Zone (late Givetian). Material. More than 120 topotypes up to ca. $30 \mathrm{~mm}$ conch diameter, including MB.C.22124.1-MB.C.22124.20, a previously unfigured syntype (paralectotype) from the Clariond Collection at Musée d'Histoire Naturelle, Paris (ds 8311, Fig. 40A), and the original of Pharciceras n. sp. in Bensaïd (1974, pl. 5, figs 3, 3a; STIPB-Bensaïd-43, old: GPI Bo 43).
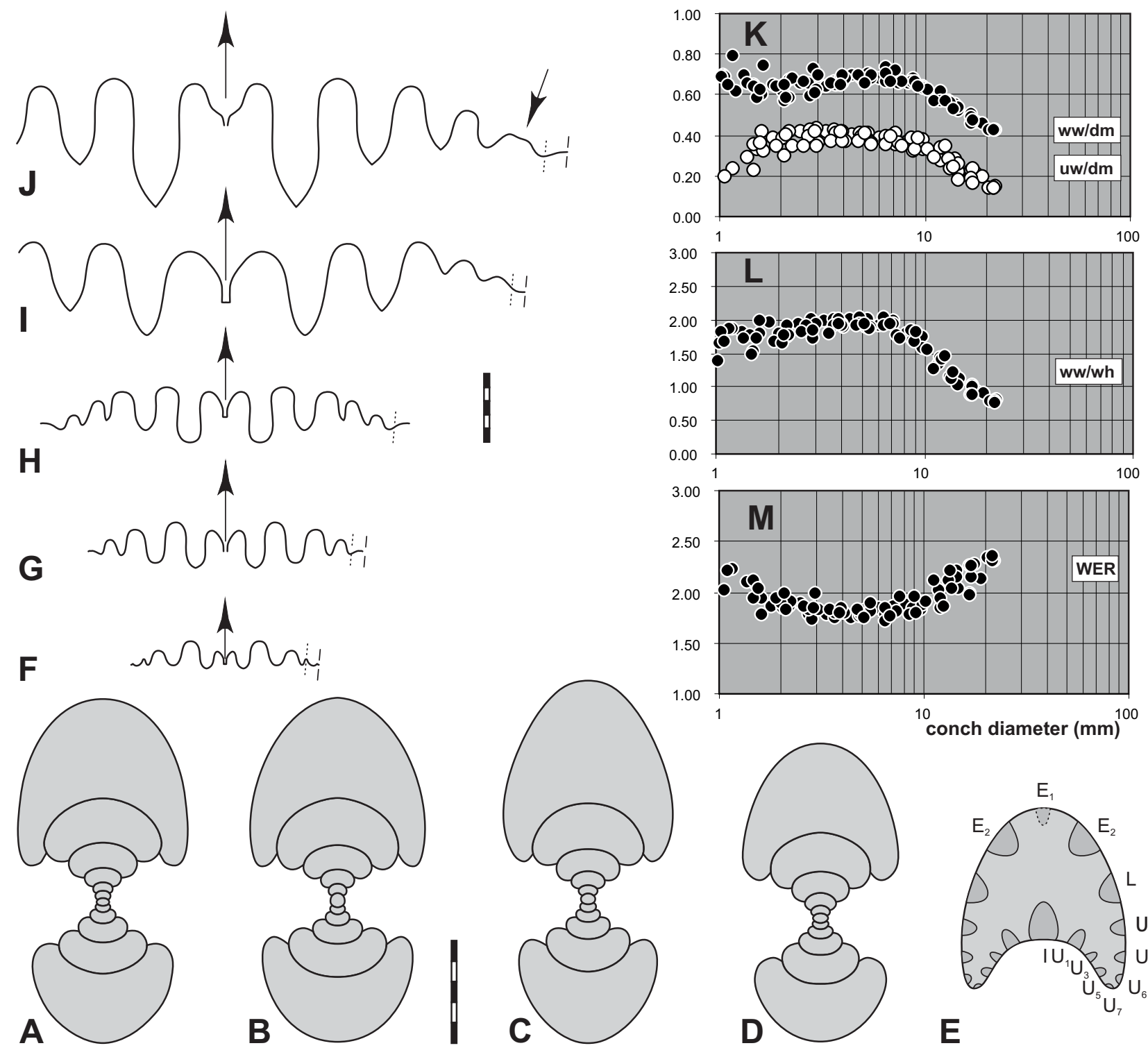

Figure 39. Stenopharciceras kseirense (Termier \& Termier, 1950), topotypes from Hassi Nebech; A-D. Cross-sections, all $\times 3$; A. MB.C.22124.1; B. MB.C.22124.2; C. MB.C.22124.3; D. MB.C.22124.4; E. Septal face of STIPB-Bensaïd-43 (old: GPI Bo 43), original of Pharciceras n. sp. of Bensaïd (1974: pl. 5, figs 3, 3a), $\times 3$; F-J. Sutures, all $\times 2.5$; F. MB.C.22124.5 at $3.5 \mathrm{~mm}$ wh; G. MB.C. 22124.6 at $6.1 \mathrm{~mm}$ wh; H. MB.C. 22124.7 at $8.5 \mathrm{~mm}$ wh; I. MB.C.22124.8 at $14.5 \mathrm{~mm}$ wh; J. MB.C.22124.9 at $20 \mathrm{~mm}$ wh; K-M. Ontogenetic development of ww/dm, uw/dm, ww/wh, and WER. 

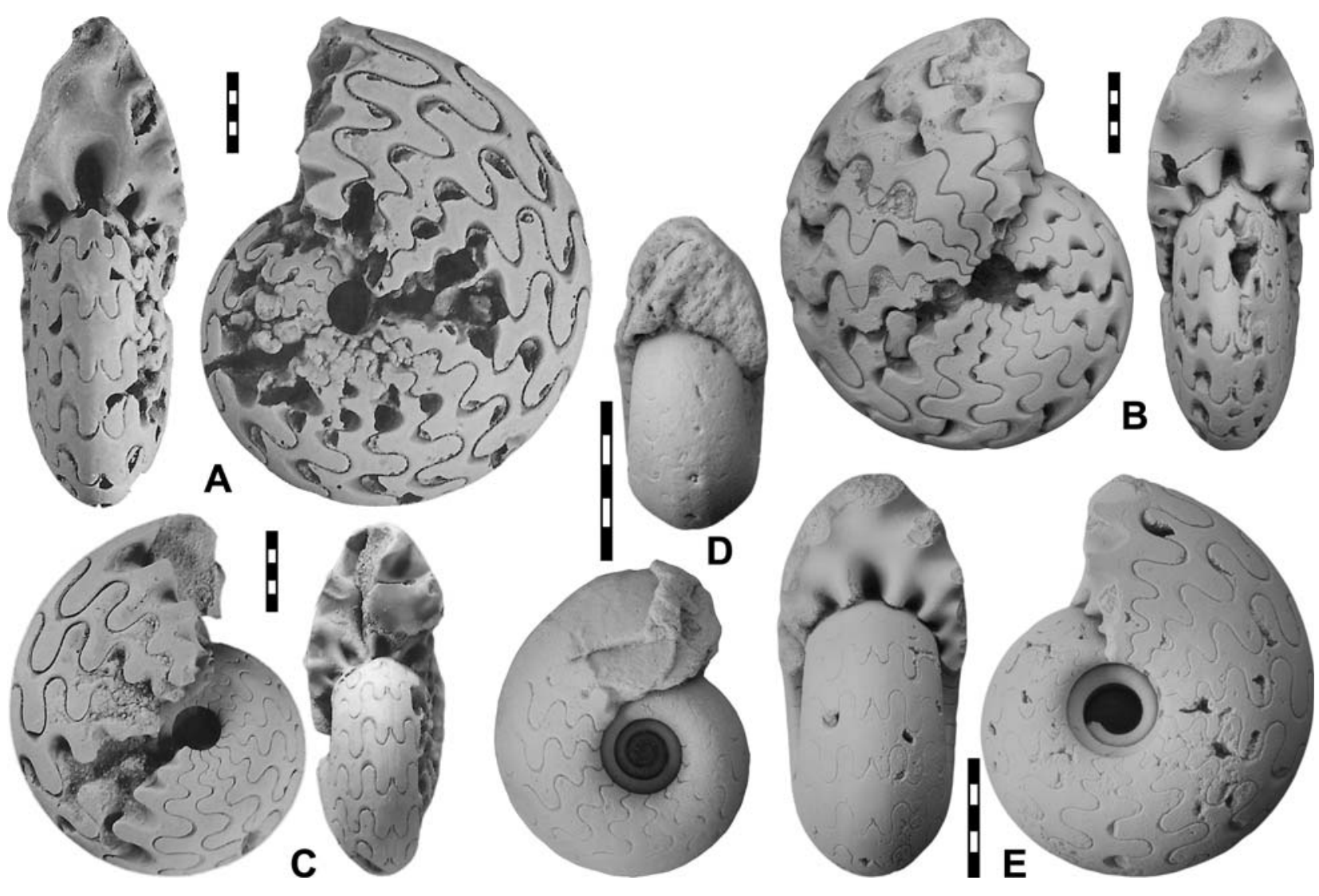

Figure 40. Stenopharciceras kseirense (Termier \& Termier, 1950) from Hassi Nebech; A. Paralectotype ds 8311, Clariond Collection, Musée d'Histoire Naturelle, Paris, $\times 2$; B. Topotype MB.C.22124.10, $\times 2$; C. Topotype MB.C.22124.11, $\times 2$; D. Topotype MB.C.22124.12, $\times$ 4; E. STIPB-Bensaïd-43 (old: GPI Bo 43), original of Pharciceras $n$. sp. of Bensaïd (1974: pl. 5, figs 3, 3a), $\times 3$.

Table 36. Conch ontogeny (Figs 39A-E, K-M) of Stenopharciceras kseirense (Termier \& Termier, 1950) from the type region.

\begin{tabular}{|c|c|c|c|}
\hline $\mathrm{dm}$ & conch shape & whorl cross-section shape & whorl expansion \\
\hline $2 \mathrm{~mm}$ & $\begin{array}{l}\text { thickly discoidal to thinly pachyconic; subevolute } \\
\text { (ww/dm }=0.55-0.65 ; \text { uw/dm } \sim 0.40 \text { ) }\end{array}$ & $\begin{array}{l}\text { moderately depressed; moderately embracing } \\
(\mathrm{ww} / \mathrm{wh}=1.70-1.80 ; \mathrm{IZR} \sim 0.20)\end{array}$ & $\begin{array}{l}\text { moderate } \\
(\text { WER } \sim 1.90)\end{array}$ \\
\hline $5 \mathrm{~mm}$ & $\begin{array}{l}\text { thinly pachyconic; subevolute } \\
(\mathrm{ww} / \mathrm{dm}=0.60-0.70 ; \mathrm{uw} / \mathrm{dm} \sim 0.40 \text { ) }\end{array}$ & $\begin{array}{l}\text { moderately depressed; moderately embracing } \\
(\mathrm{ww} / \mathrm{wh}=1.90-2.00 ; \mathrm{IZR} \sim 0.30 \text { ) }\end{array}$ & $\begin{array}{l}\text { moderate } \\
(\text { WER } \sim 1.80)\end{array}$ \\
\hline $10 \mathrm{~mm}$ & $\begin{array}{l}\text { thinly pachyconic; subevolute } \\
\text { (ww/dm } \sim 0.65 ; \mathrm{uw} / \mathrm{dm}=0.30-0.40 \text { ) }\end{array}$ & $\begin{array}{l}\text { moderately depressed; strongly embracing } \\
(\mathrm{ww} / \mathrm{wh}=1.55-1.65 ; \mathrm{IZR}=0.30-0.35)\end{array}$ & $\begin{array}{l}\text { moderate } \\
(\mathrm{WER} \sim 1.90)\end{array}$ \\
\hline $20 \mathrm{~mm}$ & $\begin{array}{l}\text { thinly discoidal; subinvolute } \\
\text { (ww/dm } \sim 0.45 ; \text { uw/dm } \sim 0.20 \text { ) }\end{array}$ & $\begin{array}{l}\text { weakly compressed; strongly embracing } \\
\text { (ww/wh }=0.80-1.00 ; \mathrm{IZR}=0.35-0.40 \text { ) }\end{array}$ & $\begin{array}{l}\text { high to very high } \\
\text { (WER }=2.15-2.35)\end{array}$ \\
\hline
\end{tabular}

Table 37. Suture formula (Figs 39E-J), conch characteristics, and ornament of Stenopharciceras kseirense (Termier \& Termier, 1950) from the type region.

$\left(E_{2} E_{1} E_{2}\right) L U_{2} U_{4}: U_{5} U_{3} U_{1} l$ at $13,5 \mathrm{~mm}$ dm (MB.C.22124.16)

$\left(E_{2} E_{1} E_{2}\right) L U_{2} U_{4} U_{6}: U_{5} U_{3} U_{1} l$ at $13,5 \mathrm{~mm}$ dm (MB.C.22124.17)

$\left(\mathrm{E}_{2} \mathrm{E}_{1} \mathrm{E}_{2}\right) L_{2} \mathrm{U}_{4} \mathrm{U}_{6}: \mathrm{U}_{7} \mathrm{U}_{5} \mathrm{U}_{3} \mathrm{U}_{1} \mathrm{l}$ at 16 and $22 \mathrm{~mm}$ dm (STIPB-Bensaïd-43, MB.C.22124.13)

$E_{1}$ lobe subparallel in juvenils, strongly funnel-shaped in adults; $E_{2}$ lobe deep and lanceolate at $\sim 25 \mathrm{~mm} \mathrm{dm}$, rounded in juveniles; sometimes plurilobation in $U_{4} U_{6}$ saddle (MB.C.22124.9)

venter narrowly rounded; umbilicus closing at the fifth whorl at steinkerns

no ribs; shallow ventrolateral furrows in juveniles, forming a cingulate venter; no mould constrictions

growth lines biconvex; Ritzstreifung in MB.C.22124.13 
Diagnosis (emend.). Conch ontogeny triphasic; first two post-embryonic whorls, until ca. $3 \mathrm{~mm} \mathrm{dm}$, with increasing umbilical width, variable whorl width rate and falling WER; late juvenile whorls until 7-8 $\mathrm{mm} \mathrm{dm}$ depressed, with nearly constant umbilical width ratio (ca. 0.40), slightly increasing ww/dm (up to ca. 0.70 ) and ww/wh ratios (up to ca. 2.00) and WER at ca. 1.80; intermediate to mature stages rapidly changing from weakly depressed to compressed, subinvolute to involute and high to very high WER (up to 2.30), with absolute reduction of the umbilical width (from ca. $14 \mathrm{~mm} \mathrm{dm}$ on) and very narrowly rounded venter. No juvenile ribs and no ventrolateral furrows on intermediate and adult whorls. Growth lines biconvex. Mature sutures with funnel-shaped, short $\mathrm{E}_{1}$ lobe, very high and narrow median saddles, very deep, lanceolate $\mathrm{E}_{2}$ lobes, very high and narrow ventral saddle, moderately deep, lanceolate $\mathrm{L}$ lobe, lanceolate, short $\mathrm{U}_{2}$ lobe, two smaller, rounded outer $U$ lobes, four internal $U$ lobes, and deep I lobe. Suture formula: $\left(E_{2} E_{1} E_{2}\right) L U_{2} U_{4} U_{6}: U_{7} U_{5} U_{3} U_{1} I$.

\section{Stenopharciceras progressum n. sp.}

Figures 41, 42

Derivation of name. From the Latin word for advanced, because of the additional U lobes.

Holotype. MB.C.22125.1, a well-preserved, fully septate mould that displays the characteristic conch shape, inner and outer sutures (Figs 41C, F, and 42B), and Ritzstreifung.

Type locality and horizon. Hassi Nebech, Section 2, SE Tafilalt, main collecting level, probably Taouzites taouzensis Zone (late Givetian). Material. 11 specimens up to ca. $34 \mathrm{~mm}$ conch diameter, including MB.C.22125.1-MB.C.22125.10.
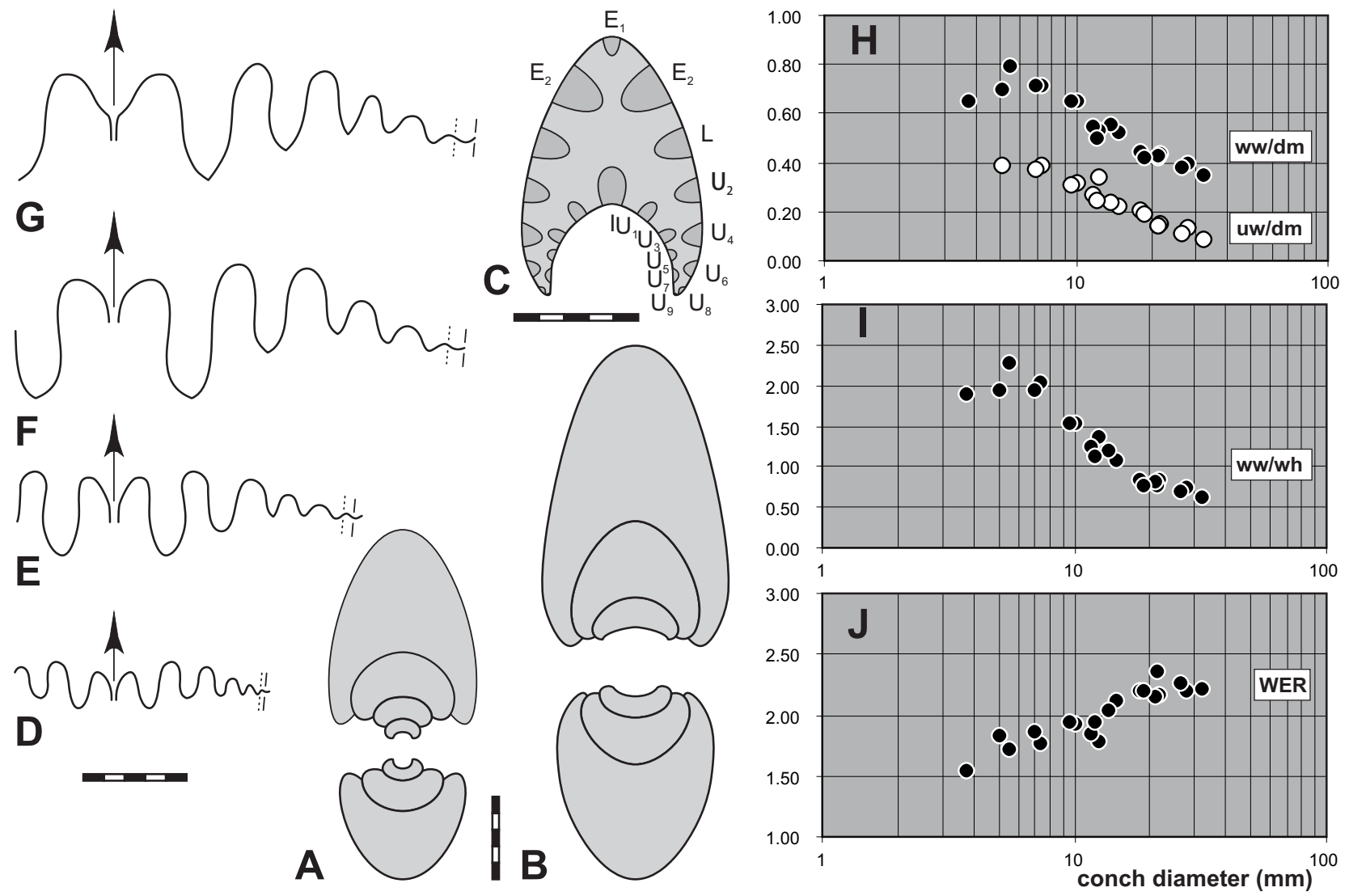

Figure 41. Stenopharciceras progressum n. sp. from Hassi Nebech; A. Cross-section of paratype MB.C.22125.2, $\times 2$; B. Crosssection of paratype MB.C.22125.3, $\times 2$; C. Septal face of holotype MB.C.22125.1, $\times 3$; D-G. Sutures, all $\times 2.5$; D. Paratype MB.C. 22125.4 at $5.2 \mathrm{~mm}$ wh; E. Paratype MB.C.22125.5 at $9.5 \mathrm{~mm}$ wh; F. Holotype MB.C.22125.1 at $14.5 \mathrm{~mm}$ wh; G. Paratype MB.C.22125.6 at $15 \mathrm{~mm}$ wh; H-J. Ontogenetic development of ww/dm, uw/dm, ww/wh, and WER.

Table 38. Conch ontogeny (Figs $41 \mathrm{~A}-\mathrm{C}, \mathrm{H}-\mathrm{J}$ ) of Stenopharciceras progressum $\mathrm{n}$. sp.

\begin{tabular}{|c|c|c|c|}
\hline$d m$ & conch shape & whorl cross-section shape & whorl expansion \\
\hline $10 \mathrm{~mm}$ & $\begin{array}{l}\text { thinly pachyconic; subevolute } \\
\text { (ww/dm } \sim 0.65 ; \mathrm{uw} / \mathrm{dm}=0.25-0.35 \text { ) }\end{array}$ & $\begin{array}{l}\text { weakly depressed; strongly embracing } \\
\text { (ww/wh } \sim 1.50 ; \text { IZR }=0.30-0.35 \text { ) }\end{array}$ & $\begin{array}{l}\text { Moderate } \\
(\text { WER } \sim 1.95)\end{array}$ \\
\hline $20 \mathrm{~mm}$ & $\begin{array}{l}\text { thinly discoidal; subinvolute } \\
\text { (ww/dm } \sim 0.45 ; \text { uw/dm } \sim 0.20 \text { ) }\end{array}$ & $\begin{array}{l}\text { weakly compressed; strongly embracing } \\
\text { (ww/wh } \sim 0.85 \mathrm{IZR}=0.35-0.40 \text { ) }\end{array}$ & $\begin{array}{l}\text { high } \\
\text { (WER 2.15) }\end{array}$ \\
\hline $30 \mathrm{~mm}$ & $\begin{array}{l}\text { thinly discoidal; involute } \\
\text { (ww/dm } \sim 0.35 ; \text { uw/dm } \sim 0.10 \text { ) }\end{array}$ & $\begin{array}{l}\text { weakly compressed; strongly embracing } \\
\text { (ww/wh } \sim 0.70 \mathrm{IZR}=0.35-0.40 \text { ) }\end{array}$ & $\begin{array}{l}\text { high } \\
\text { (WER 2.25) }\end{array}$ \\
\hline
\end{tabular}


Table 39. Suture formula (Figs $41 \mathrm{C}-\mathrm{G}$ ), conch characteristics, and ornament of Stenopharciceras progressum $\mathrm{n}$. sp.

\section{$\left(\mathrm{E}_{2} \mathrm{E}_{1} \mathrm{E}_{2}\right) L U_{2} \mathrm{U}_{4} \mathrm{U}_{6}: \mathrm{U}_{5} \mathrm{U}_{3} \mathrm{U}_{1} \mathrm{l}$ at $12 \mathrm{~mm}$ dm (MB.C.22125.9)}

\section{$\left(E_{2} E_{1} E_{2}\right) L U_{2} U_{4} U_{6} U_{8}: U_{7} U_{5} U_{3} U_{1}$ I at $16,5 \mathrm{~mm}$ dm (holotype)}

$E_{1}$ lobe subparallel in juveniles, funnel-shaped in adults; $E_{2}$ lobe deep and lanceolate at $\sim 27 \mathrm{~mm} \mathrm{dm}$, rounded in juveniles; $U_{2}$ lobe often hook-shaped; one outer $\mathrm{U}$ lobe more than in Stenopharciceras kseirense
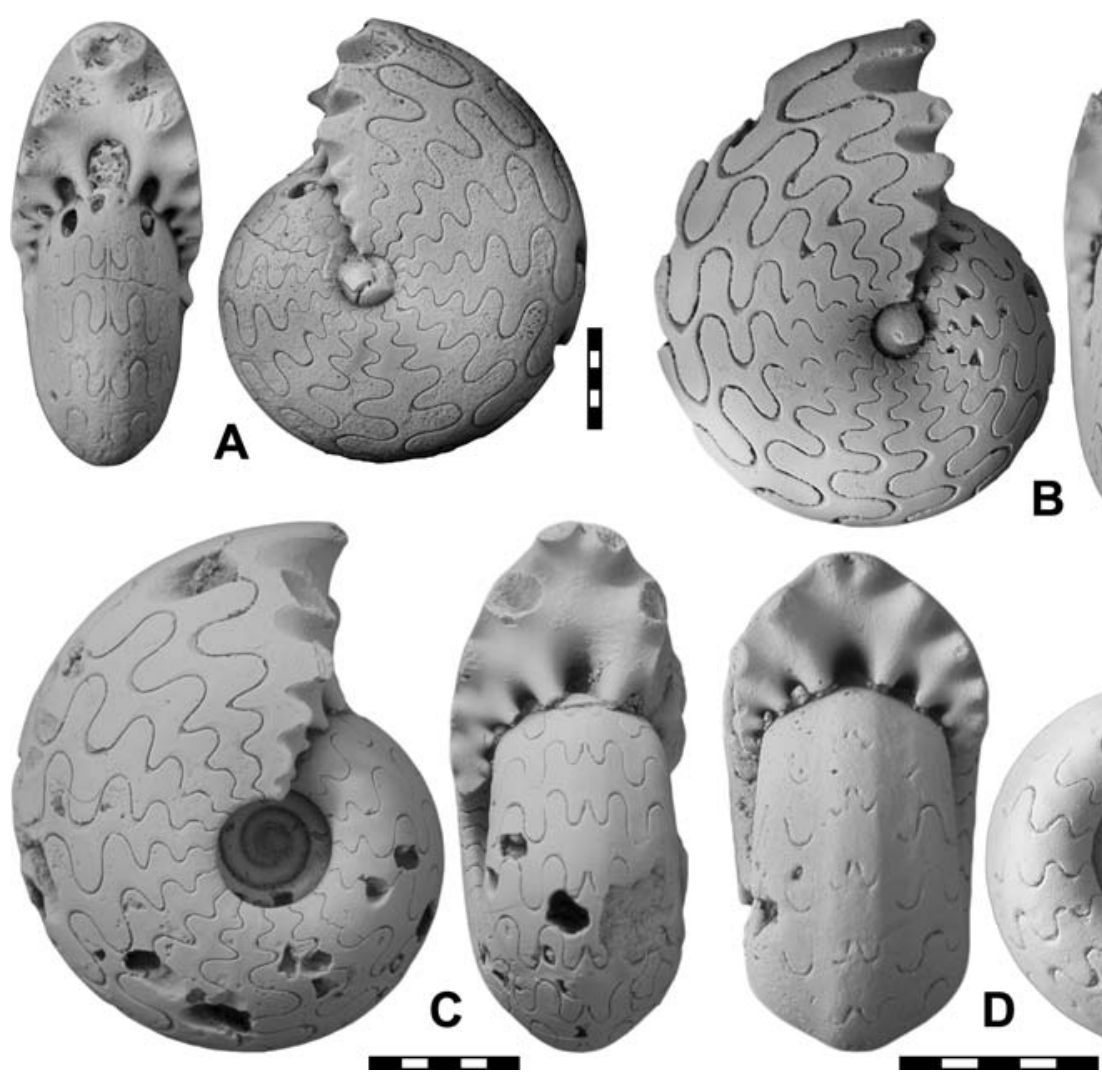

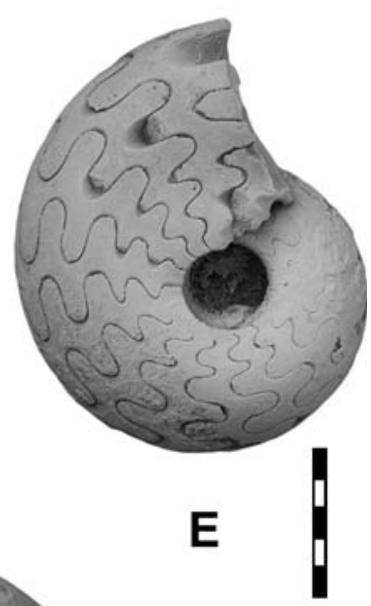

Figure 42. Stenopharciceras progressum n. sp. from Hassi Nebech; A. Paratype MB.C.22125.10, $\times 2$; B. Holotype MB.C.22125.1, $\times 2$; C. Paratype MB.C.22125.5, ×3; D. Paratype MB.C.22125.4; $\times 4$, E. Paratype MB.C.22125.7, $\times 3$.

Diagnosis. Conch shape and ontogeny as in St. kseirense. Growth lines biconvex, with moderately high ventrolateral salient. Mature sutures with strongly funnel-shaped, wide $\mathrm{E}_{1}$ lobe, diverging in the late part, high median saddle, very deep, lanceolate $\mathrm{E}_{2}$ lobe, high and very narrow $\mathrm{E}_{2} \mathrm{~L}$ saddle, moderately deep, lanceolate $\mathrm{L}$ lobe, lanceolate $\mathrm{U}_{2}$ lobe, three further, rounded outer $\mathrm{U}$ lobes separated by small saddles becoming lower towards the umbilicus, five, gradually deeper internal $U$ lobes, and deep, narrow I lobe. Suture formula: $\left(E_{2} E_{1} E_{2}\right) L_{2} U_{4} U_{6} U_{8}: U_{9} U_{7} U_{5} U_{3} U_{1} I$.

\section{Pluripharciceras n. gen.}

Type species. Synpharciceras plurilobatum Petter, 1959.

Derivation of name. After the name of the type species and due to the addition of U lobes in comparison to Stenopharciceras.

Diagnosis. Early whorls increasingly depressed, subevolute and with broadly rounded venter, intermediate to adult conchs gradually moderately to strongly compressed and subinvolute to involute, with absolute reduction of the umbilical width, high to very high whorl expansion and narrowly rounded venter. Growth lines strongly biconvex, with projecting ventrolateral salient. Mature suture formula: $\left(\mathrm{E}_{2} \mathrm{E}_{1} \mathrm{E}_{2}\right)$ $\mathrm{LU}_{2} \mathrm{U}_{4} \mathrm{U}_{6} \mathrm{U}_{8} \mathrm{U}_{10}: \mathrm{U}_{11} \mathrm{U}_{9} \mathrm{U}_{7} \mathrm{U}_{5} \mathrm{U}_{3} \mathrm{U}_{1} \mathrm{I}$ to $\left(\mathrm{E}_{2} \mathrm{E}_{1} \mathrm{E}_{2}\right) \mathrm{LU}_{2} \mathrm{U}_{4} \mathrm{U}_{6} \mathrm{U}_{8} \mathrm{U}_{10} \mathrm{U}_{12}: \mathrm{U}_{13} \mathrm{U}_{11} \mathrm{U}_{9} \mathrm{U}_{7} \mathrm{U}_{5} \mathrm{U}_{3} \mathrm{U}_{1} \mathrm{I}$.

Included species

Synpharciceras plurilobatum Petter, 1959

Pluripharciceras orbis $\mathrm{n}$. gen. n. sp.

?Pharciceras arenicum in House et al. (1985)

Stratigraphic range and geographic distribution. Late Givetian of the Tafilalt, possibly also of the Montagne Noire. 


\section{Pluripharciceras plurilobatum (Petter, 1959)}

Figures 43B-F, H-L, 44, cf. 43A, G

Lectotype. The original of Petter (1959, pl. 8, figs 5, 5a), first illustrated by Termier \& Termier (1950, pl. 151, figs 16-18) as Pharciceras taouzensis, Clariond Collection, Paris Natural History Museum, re-illustrated in Figure 44A, is here designated as lectotype. It is a specimen with a part of the last whorl broken off, but showing the typical conch shape and sutures, including a septal face with all inner lobes.

Type locality and horizon. Oued Kseir = Hassi Nebech area, SE Tafilalt, main collecting level, probably Taouzites taouzensis Zone (late Givetian).

Material. Ca. 65 specimens up to $37.5 \mathrm{~m}$ conch diameter, including the lectotype, 18 topotypes (MB.C.22126.1-MB.C.22126.18), and two specimens identified as cf. plurilobatum (MB.C.22127.1-MB.C.22127.2).

Diagnosis (emend.). First two whorls until ca. $2 \mathrm{~mm}$ dm with rapidly decreasing whorl expansion rate (down to ca. 1.9-1.85) and rising umbilical width ratio (uw/dm up to 0.35 ); until $8-9 \mathrm{~mm}$ dm with nearly constant WER, increasingly depressed (ww/dm up to ca 0.60 , ww/wh up to 1.70), narrowly rounded venter and subevolute; intermediate to mature whorls increasingly less depressed to compressed (from ca. $15 \mathrm{~mm} \mathrm{dm}$ ),

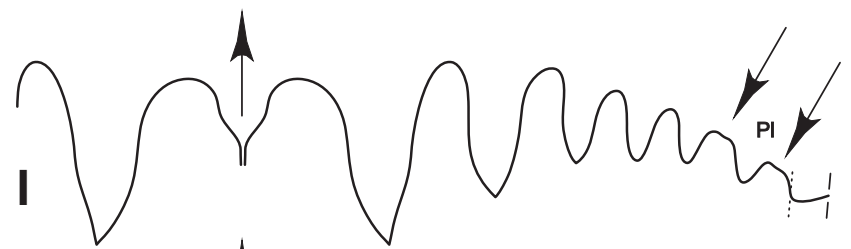

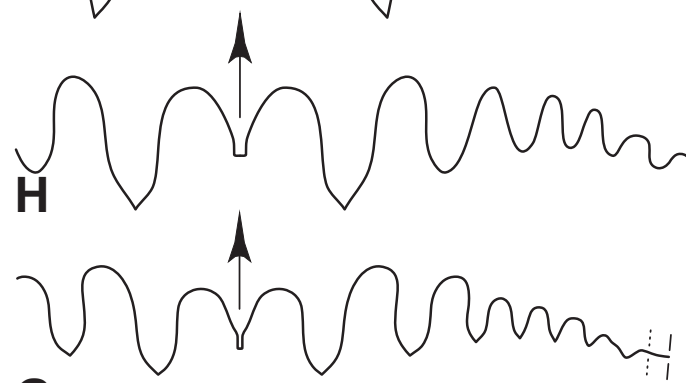

G

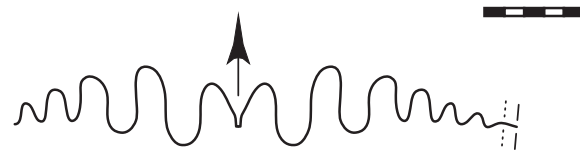

$\mathbf{F}$
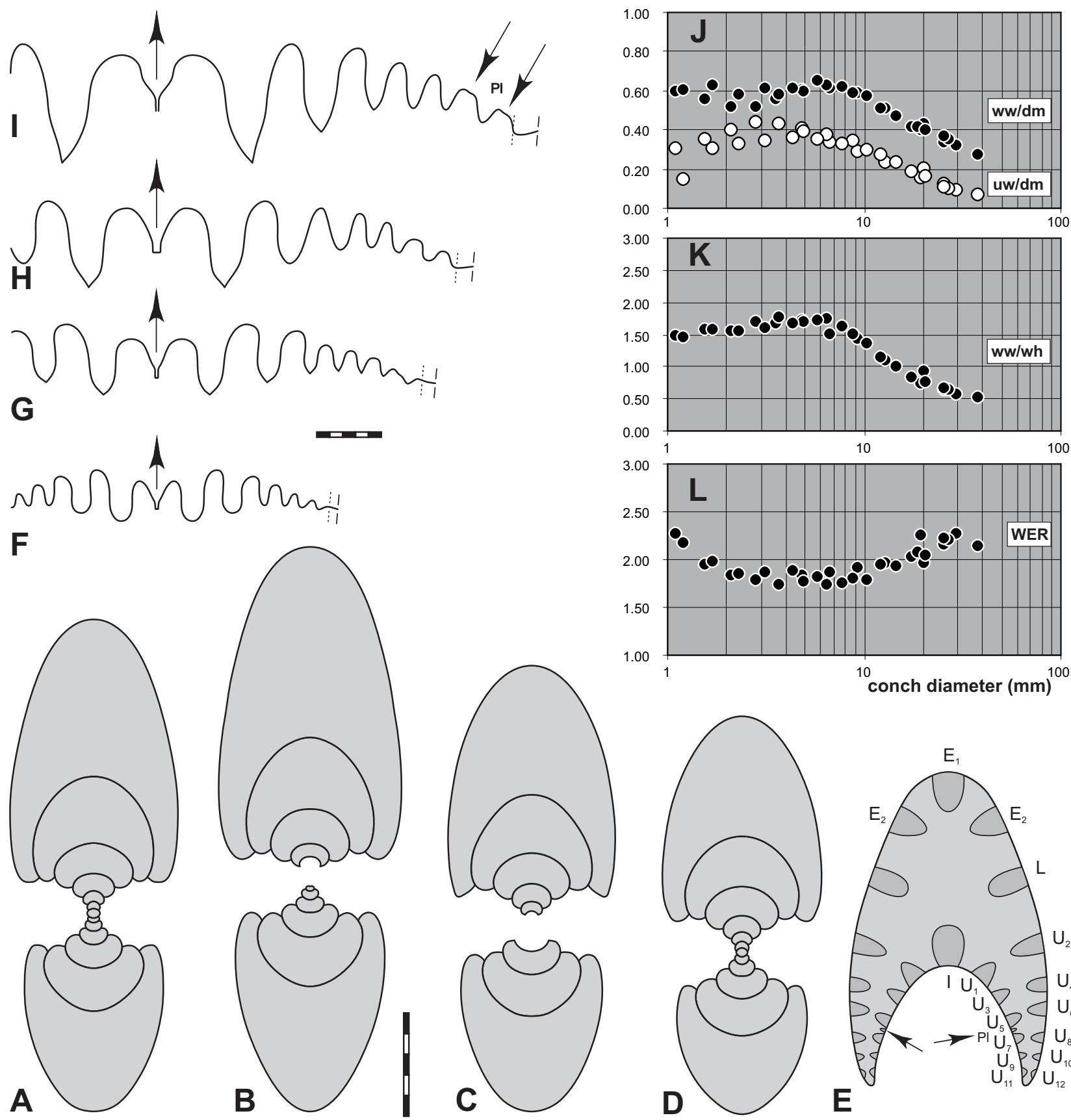

Figure 43. Pluripharciceras plurilobatum (Petter, 1959), topotypes from Hassi Nebech; A-D. Cross-sections, all $\times 3$; A. cf. specimen MB.C.22127.1; B. MB.C.22126.1; C. MB.C.22126.2; D. MB.C.22126.3; E. Septal face of MB.C.22126.4, $\times 3$; F-I. Sutures, all $\times 2.5$; F. MB.C.22126.5 at $9.3 \mathrm{~mm}$ wh; G. cf. specimen MB.C. 22127.2 at $15.5 \mathrm{~mm}$ wh; H. MB.C.22126.6 at $16.3 \mathrm{~mm}$ wh; I. MB.C.22126.11 at $21 \mathrm{~mm}$ wh; J-L. Ontogenetic development of ww/dm, uw/dm, ww/wh, and WER. 


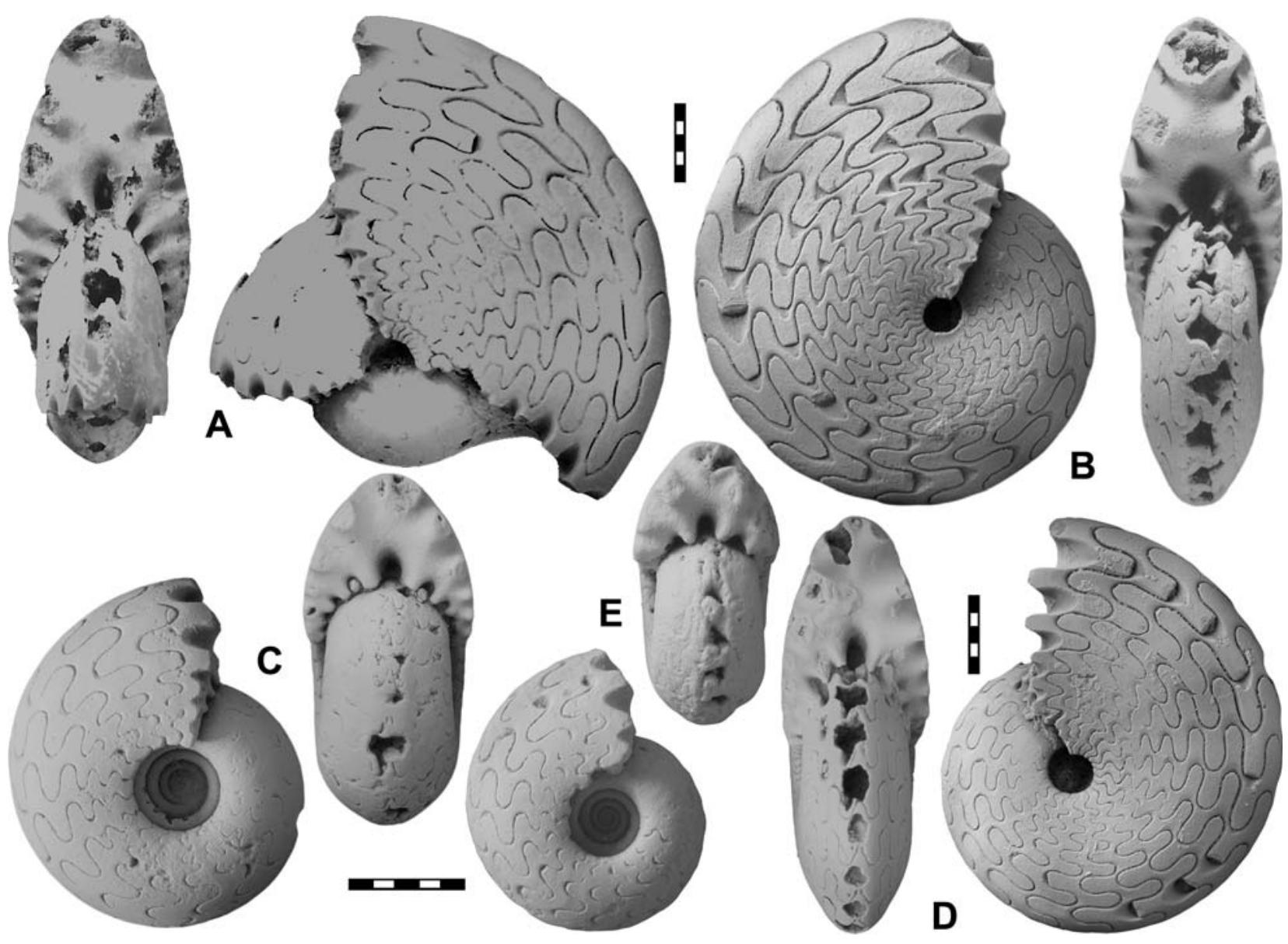

Figure 44. Pluripharciceras plurilobatum (Petter, 1959) from Hassi Nebech; A. Lectotype, original of Petter (1959: pl. 8, figs 5, 5a), Clariond Collection, Musée d'Histoire Naturelle, Paris, $\times 2$; B. Topotype MB.C.22126.7, $\times 2$; C. Topotype MB.C.22126.9, $\times 3$; D. Topotype MB.C.22126.8, $\times 2$; E. Topotype MB.C.22126.10, $\times 3$.

Table 40. Conch ontogeny (Figs 43A-E, J-L) of Pluriphaciceras plurilobatum (Petter, 1959) from the type locality.

\begin{tabular}{|c|c|c|c|}
\hline $\mathrm{dm}$ & conch shape & whorl cross-section shape & whorl expansion \\
\hline $2 \mathrm{~mm}$ & $\begin{array}{l}\text { thickly discoidal; subevolute } \\
\text { (ww/dm =0.50-0.60; uw/dm } \sim 0.35 \text { ) }\end{array}$ & $\begin{array}{l}\text { moderately depressed; moderately embracing } \\
\text { (ww/wh } \sim 1.60 ; \text { IZR } \sim 0.25)\end{array}$ & $\begin{array}{l}\text { moderate } \\
(\text { WER } \sim 1.85)\end{array}$ \\
\hline $5 \mathrm{~mm}$ & $\begin{array}{l}\text { thinly pachyconic; subevolute } \\
\text { (ww/dm }=0.60-0.70 ; \text { uw/dm } \sim 0.35 \text { ) }\end{array}$ & $\begin{array}{l}\text { moderately depressed; moderately embracing } \\
\text { ( } w w / w h \sim 1.70 ; \text { IZR } \sim 0.30 \text { ) }\end{array}$ & $\begin{array}{l}\text { moderate } \\
(\text { WER } \sim 1.85)\end{array}$ \\
\hline $20 \mathrm{~mm}$ & $\begin{array}{l}\text { thinly discoidal; subinvolute } \\
\text { (ww/dm =0.40-0.50; uw/dm } \sim 0.15 \text { ) }\end{array}$ & $\begin{array}{l}\text { weakly compressed; strongly embracing } \\
\text { ( } \mathrm{ww} / \mathrm{wh}=0.75-0.85 ; \mathrm{IZR}=0.35-0.40 \text { ) }\end{array}$ & $\begin{array}{l}\text { high } \\
(\text { WER }=2.05-2.25)\end{array}$ \\
\hline $30 \mathrm{~mm}$ & $\begin{array}{l}\text { extremely discoidal; involute } \\
\text { ( } w w / d m=0.30-0.35 ; \text { uw/dm } \sim 0.10)\end{array}$ & $\begin{array}{l}\text { weakly compressed; strongly embracing } \\
\text { (ww/wh } \sim 0.60 ; \text { IZR } \sim 0.40 \text { ) }\end{array}$ & $\begin{array}{l}\text { very high } \\
\text { (WER 2.30) }\end{array}$ \\
\hline
\end{tabular}

Table 41. Suture formula (Figs 43E-I), conch characteristics, and ornament of Pluriphaciceras plurilobatum (Petter, 1959) from the type locality.

$\left(E_{2} E_{1} E_{2}\right) L U_{2} U_{4} U_{6} U_{8}: U_{7} U_{5} U_{3} U_{1} l$ at $15 \mathrm{~mm}$ dm (MB.C.22126.16)
$\left(E_{2} E_{1} E_{2}\right) L U_{2} U_{4} U_{6} U_{8} U_{10}: U_{9} U_{7} U_{5} U_{3} U_{1} l$ at $19 \mathrm{~mm}$ dm (MB.C.22126.5)
$\left(E_{2} E_{1} E_{2}\right) L U_{2} U_{4} U_{6} U_{8} U_{10} U_{12}: U_{11} U_{9} U_{7} P I U_{5} U_{3} U_{1} l$ at $28 \mathrm{~mm}$ dm (MB.C.22126.4)
$E_{1}$ lobe funnel-shaped, median saddle $\sim 50-60$ \% depth of $E_{2}$ lobe; $E_{2}$ lobe deep and lanceolate; plurilobation by subdivision of the dorsolat-
eral $U$ saddels close to the umbilicus externally and/or or internally
conch discoconic; venter narrowly rounded; umbilicus closing at steinkerns at about $20 \mathrm{~mm} \mathrm{dm}$
no ribs; ventrolateral furrows until ca. $6.5 \mathrm{~mm}$ dm; no mould constrictions
growth lines rectiradiate, strongly biconvex, with projecting ventrolateral salient


finally strongly compressed, with narrowly rounded venter, closure of the umbilicus by overlap of mould whorls over the umbilicus, and high to very high WER (up to 2.35). Growth lines strongly biconvex, no ribbing, no marked ventrolateral furrows. Adult sutures with short, strongly funnel-shaped $\mathrm{E}_{1}$ lobe, wide, moderately high median saddle, very deep, lanceolate $\mathrm{E}_{2}$ lobe, high and narrow $\mathrm{E}_{2} \mathrm{~L}$ saddle, moderately deep, lanceolate $\mathrm{L}$ lobe, high $\mathrm{LU}_{2}$ saddle, two pointed, two narrowly rounded, and one widely rounded U lobes, separated by descending saddles, which become wider towards the umbilicus; inner suture with six U lobes and a very deep, narrow I lobe; plurilobes common. Suture formula: $\left(\mathrm{E}_{2} \mathrm{E}_{1} \mathrm{E}_{2}\right) \mathrm{LU}_{2} \mathrm{U}_{4} \mathrm{U}_{6} \mathrm{U}_{8} \mathrm{U}_{10} \mathrm{U}_{12}: \mathrm{U}_{11} \mathrm{U}_{9} \mathrm{U}_{7} \mathrm{U}_{5} \mathrm{U}_{3} \mathrm{U}_{1} \mathrm{I}$

\section{Pluripharciceras orbis n. sp.}

Figures 45, 46

Derivation of name. After the Latin word orbis = disc; due to the thinly discoidal conch shape

Holotype. MB.C.22128.1 (Figs 45A, D, and 46A)

Type locality and horizon. Hassi Nebech, Section 2, SE Tafilalt, main collecting level, probably Taouzites taouzensis Zone (late Givetian). Material. Four specimens up to ca. $17 \mathrm{~mm}$ conch diameter (MB.C.22128.1-MB.C.22128.4).

Diagnosis. WER falling sharply to ca. 2.0 at the end of the second whorl; first four whorls, up to ca. $7 \mathrm{~mm} \mathrm{dm}$, increasingly subevolute (uw/ $\mathrm{dm}$ up to ca. 0.35 ), thinly pachyconic and moderately depressed (ww/wh ratios reach 1.6); intermediate stages gradually more involute (uw/dm

D

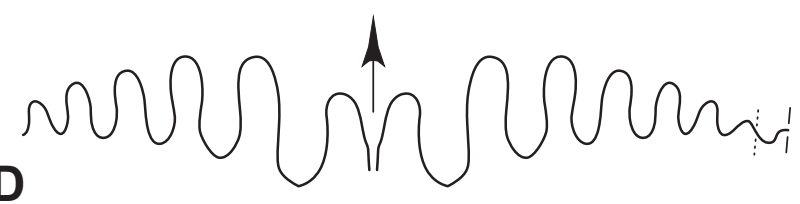

C

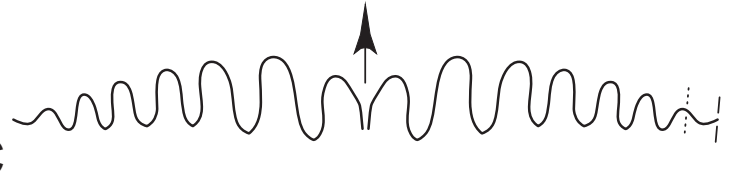

A

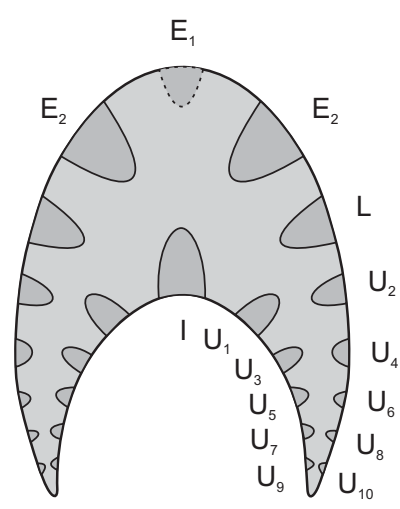

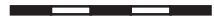

B

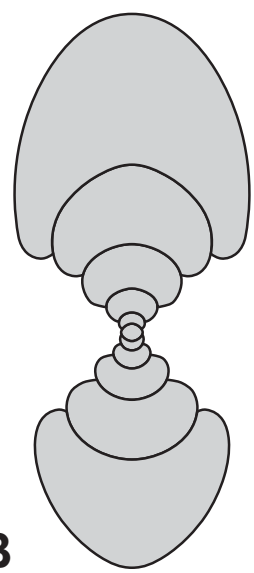

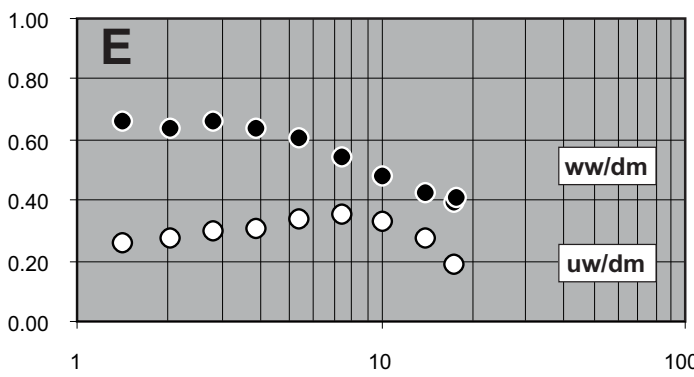
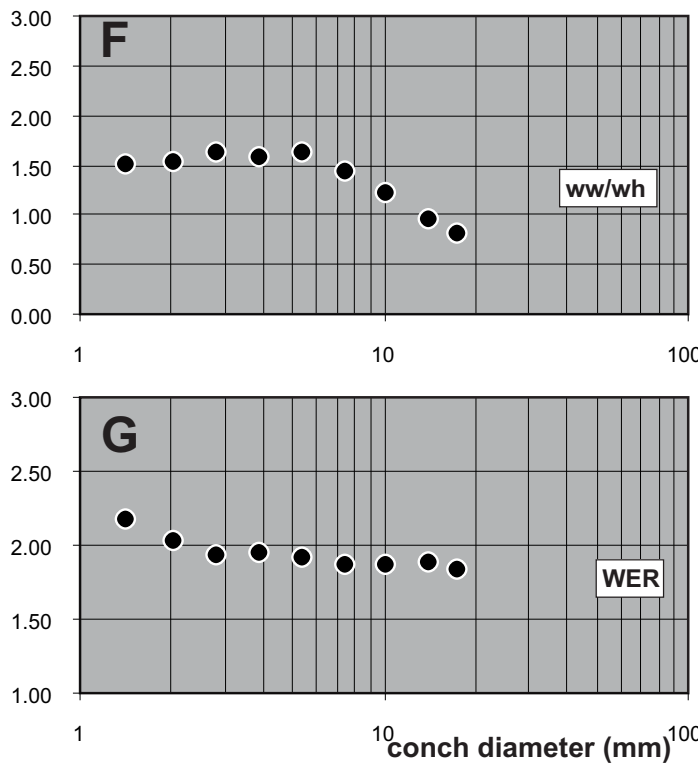

Figure 45. Pluripharciceras orbis n. sp. from Hassi Nebech; A. Septal face of holotype MB.C.22128.1, $\times 5$; B. Cross-section of paratype MB.C.22128.2, $\times 4$; C. Suture of paratype MB.C.22128.3 at $6.7 \mathrm{~mm}$ wh, $\times 4$; D. Suture of holotype MB.C.22128.1 at $8.4 \mathrm{~mm}$ wh, $\times 4$; E-G. Ontogenetic development of ww/dm, uw/dm, ww/wh, and WER of all available specimens.

Table 42. Conch ontogeny (Figs 45A, B, E-G) of Pluripharciceras orbis n. sp.

\begin{tabular}{llll}
\hline $\mathrm{dm}$ & conch shape & whorl cross-section shape & whorl expansion \\
\hline $2 \mathrm{~mm}$ & thinly pachyconic; subinvolute & moderately depressed; strongly embracing & moderate \\
& $($ ww/dm $\sim 0.65 ;$ uw/dm $\sim 0.30)$ & $($ ww/wh $\sim 1.55 ;$ IZR $\sim 0.30)$ & $($ WER $\sim 2.00)$ \\
$5 \mathrm{~mm}$ & thickly discoidal; subevolute & moderately depressed; moderately embracing & moderate \\
& $($ ww/dm $\sim 0.60 ;$ uw $/ \mathrm{dm} \sim 0.35)$ & $($ ww/wh $\sim 1.65 ;$ IZR $\sim 0.25)$ & $($ WER $\sim 1.90)$ \\
$15 \mathrm{~mm}$ & thinly discoidal; subinvolute & weakly compressed; strongly embracing & moderate \\
& $($ ww/dm $\sim 0.40 ;$ uw/dm $\sim 0.20)$ & $($ ww/wh $=0.80-0.90 ;$ IZR $\sim 0.45)$ & $($ WER $\sim 1.85)$ \\
\end{tabular}


Table 43. Suture formula (Figs 45A, C, D), conch characteristics, and ornament of Pluripharciceras orbis $\mathrm{n}$. $\mathrm{sp}$.

$\left(E_{2} E_{1} E_{2}\right) L U_{2} U_{4} U_{6} U_{8}: U_{7} U_{5} U_{3} U_{1} l$ at $15 \mathrm{~mm} d m(M B . C .22128 .2)$

$\left(E_{2} E_{1} E_{2}\right) L U_{2} U_{4} U_{6} U_{8} U_{10}: U_{9} U_{7} U_{5} U_{3} U_{1} l$ at $17 \mathrm{~mm}$ dm (holotype))

$E_{1}$ lobe short, narrow, funnel-shaped; flank saddles self-similar

venter narrowly rounded, subinvolute

no ribs; no furrows; no mould constrictions

growth lines not visible

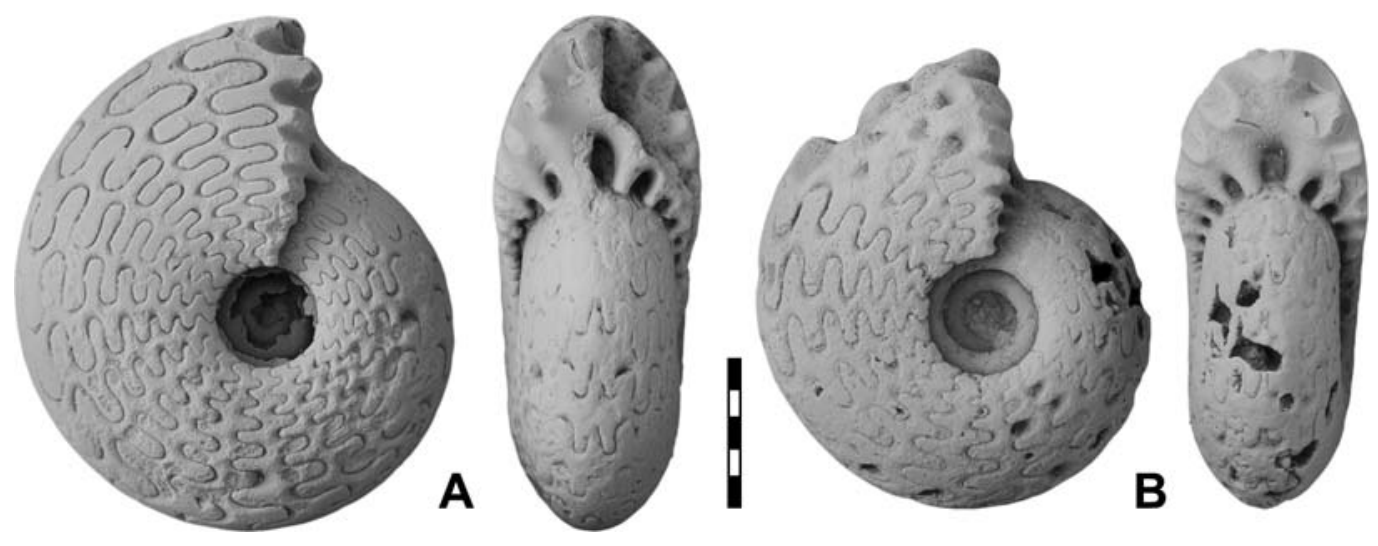

Figure 46. Pluripharciceras orbis n. sp. from Hassi Nebech; A. Holotype MB.C.22128.1, ×3; B. Paratype MB.C.22128.2, ×3.

falling below 0.20), with slight absolute reduction of the umbilicus by overlap of the moderately expanding whorls (WER $=$ ca. 1.85 ), less depressed to compressed (from ca. $15 \mathrm{~mm} \mathrm{dm}$ ), and thinly discoidal, with gently rounded flanks and venter. Sutures at $17 \mathrm{~mm}$ dm with funnelshaped, shortened, narrow $\mathrm{E}_{1}$ lobe, moderately high median saddle, deep $\mathrm{E}_{2}$ lobe, narrow and high ventral saddle, $\mathrm{L}$ and five outer $\mathrm{U}$ lobes of ascending depth towards the umbilicus, separated by narrow saddles of descending height, five internal U lobes, and deep, narrow I lobe. Suture formula: $\left(\mathrm{E}_{2} \mathrm{E}_{1} \mathrm{E}_{2}\right) \mathrm{LU}_{2} \mathrm{U}_{4} \mathrm{U}_{6} \mathrm{U}_{8} \mathrm{U}_{10}: \mathrm{U}_{9} \mathrm{U}_{7} \mathrm{U}_{5} \mathrm{U}_{3} \mathrm{U}_{1} \mathrm{I}$.

\section{Meropharciceras Becker \& House, 1993}

Type species. Beloceras? disciforme Bensaid, 1974.

\section{Meropharciceras disciforme (Bensaïd, 1974)}

Figures 47, 48

Holotype. STIPB-Bensaïd-53 (old: GPI Bo 53), original of Bensaïd (1974, pl. 5, fig. 13), re-illustrated by Becker \& House (1993), and, partly (suture), by Korn \& Klug (2002).

Type locality and horizon. Hassi Nebech, Section 2, SE Tafilalt; Late Givetian, probably Petteroceras errans Zone, as at Ouidane Chebbi (Belka et al. 1999).

Material. The holotype and six topotypes up to ca. $35 \mathrm{~mm}$ conch diameter (MB.C.22129.1-MB.C.22129.6).

Table 44. Conch ontogeny (Figs 47A-D, I-K) of Meropharciceras disciforme (Bensaï, 1974).

\begin{tabular}{|c|c|c|c|}
\hline $\mathrm{dm}$ & conch shape & whorl cross-section shape & whorl expansion \\
\hline $2 \mathrm{~mm}$ & $\begin{array}{l}\text { thinly pachyconic; subevolute } \\
\text { (ww/dm } \sim 0.65 ; \text { uw/dm } \sim 0.35 \text { ) }\end{array}$ & $\begin{array}{l}\text { moderately depressed; moderately embracing } \\
(\mathrm{ww} / \mathrm{wh} \sim 1.75 ; \mathrm{IZR} \sim 0.30)\end{array}$ & $\begin{array}{l}\text { moderate } \\
(\text { WER } \sim 1.85)\end{array}$ \\
\hline $5 \mathrm{~mm}$ & $\begin{array}{l}\text { thinly pachyconic; subevolute } \\
\text { (ww/dm } \sim 0.60 ; \mathrm{uw} / \mathrm{dm} \sim 0.35 \text { ) }\end{array}$ & $\begin{array}{l}\text { moderately depressed; strongly embracing } \\
\text { (ww/wh } \sim 1.70 ; \text { IZR } \sim 0.40 \text { ) }\end{array}$ & $\begin{array}{l}\text { low } \\
\text { (WER 1.70) }\end{array}$ \\
\hline $10 \mathrm{~mm}$ & $\begin{array}{l}\text { thickly discoidal; subinvolute } \\
\text { (ww/dm } \sim 0.55 ; \text { uw } / \mathrm{dm} \sim 0.30 \text { ) }\end{array}$ & $\begin{array}{l}\text { weakly depressed; strongly embracing } \\
\text { (ww/wh } \sim 1.40 \text {; IZR } \sim 0.45 \text { ) }\end{array}$ & $\begin{array}{l}\text { low } \\
(\text { WER } \sim 1.70)\end{array}$ \\
\hline $15 \mathrm{~mm}$ & $\begin{array}{l}\text { thickly discoidal; subinvolute } \\
\text { (ww/dm } \sim 0.50 ; \text { uw/dm } \sim 0.20 \text { ) }\end{array}$ & $\begin{array}{l}\text { weakly depressed; strongly embracing } \\
\text { (ww/wh } \sim 1.05 ; \text { IZR } \sim 0.45 \text { ) }\end{array}$ & $\begin{array}{l}\text { moderate } \\
(\text { WER } \sim 1.80)\end{array}$ \\
\hline $55 \mathrm{~mm}$ & $\begin{array}{l}\text { extremely discoidal;involute } \\
\text { (ww/dm } \sim 0.27 ; \text { uw/dm } \sim 0.05)\end{array}$ & $\begin{array}{l}\text { strongly compressed; strongly embracing } \\
\text { (ww/wh } \sim 0.45 ; \text { IZR } \sim 0.45 \text { ); }\end{array}$ & $\begin{array}{l}\text { high } \\
\text { (WER 2.25) }\end{array}$ \\
\hline
\end{tabular}


Table 45. Suture formula (Figs 47D-H), conch characteristics, and ornament of Meropharciceras disciforme (Bensaïd, 1974).

\section{$\left(E_{2} E_{1} E_{2}\right) L U_{2} U_{4} U_{6} U_{8}: U_{7} U_{5} U_{3} U_{1} I$ at $8 m m$ dm (MB.C.22129.6)}

$\left(E_{2} E_{1} E_{2}\right) L_{2} U_{4} U_{6} U_{8} U_{10} U_{12}: U_{11} U_{9} U_{7} U_{5} U_{3} U_{1} l$ at ca. 11 and $13 \mathrm{~mm} \mathrm{dm} \mathrm{(MB.C.22129.6)} \mathrm{MB.C.22129.4)}$

$\left(E_{2} E_{1} E_{2}\right) L U_{2} U_{4} U_{6} U_{8} U_{10} U_{12} U_{14}: U_{13} U_{11} U_{9} U_{7} U_{5} U_{3} U_{1}$ l at ca. $33 \mathrm{~mm} \mathrm{dm} \mathrm{(MB.C.22129.5)}$

$\left(E_{2}\left(E_{3}\right) E_{1}\left(E_{3}\right) E_{2}\right) L U_{2} U_{4} U_{6} U_{8} U_{10} U_{12} U_{14} U_{16}: U_{15} U_{13} U_{11} U_{9} U_{7} U_{5} U_{3} U_{1}$ l at ca. $55 \mathrm{~mm}$ dm (holotype)

$E_{1}$ broad and funnel-shaped, trifid, with incipient $E_{3}$ lobe at latest maturity; $E_{2}$ lobe, $L, U_{2}$ and $U_{4}$ lobe deep and lanceolate; all saddles narrowly rounded

venter narrowly rounded; umbilicus closing by whorl overlap

no ribs; no ventrolateral furrows; no mould constrictions

growth lines biconvex, with relative low ventrolateral salient

Diagnosis (emend.). First four whorls, until ca. $4 \mathrm{~mm} \mathrm{dm}$, moderately depressed, with gradually opening umbilicus (uw/dm up to 0.40 ), nearly constant ww/wh ratios (around 1.70) and declining WER (down to 1.70); 5th and 6th whorl gradually less depressed (ww/dm $=0.55$ at $10 \mathrm{~mm}$ $\mathrm{dm}$ ) and with decreasing umbilical width ratio (uw/dm down to 0.20 at $15 \mathrm{~mm} \mathrm{dm})$; from ca. the 7 th whorl $(17 \mathrm{~mm} \mathrm{dm})$ weakly to strongly compressed, with higher whorls (WER up to $>2.20$ ), with narrowly rounded venter, and with almost closure of the umbilicus by whorl overlap over the umbilical space. No ribbing of early whorls, no ventrolateral furrows. Growth lines biconvex. Mature sutures with very small and

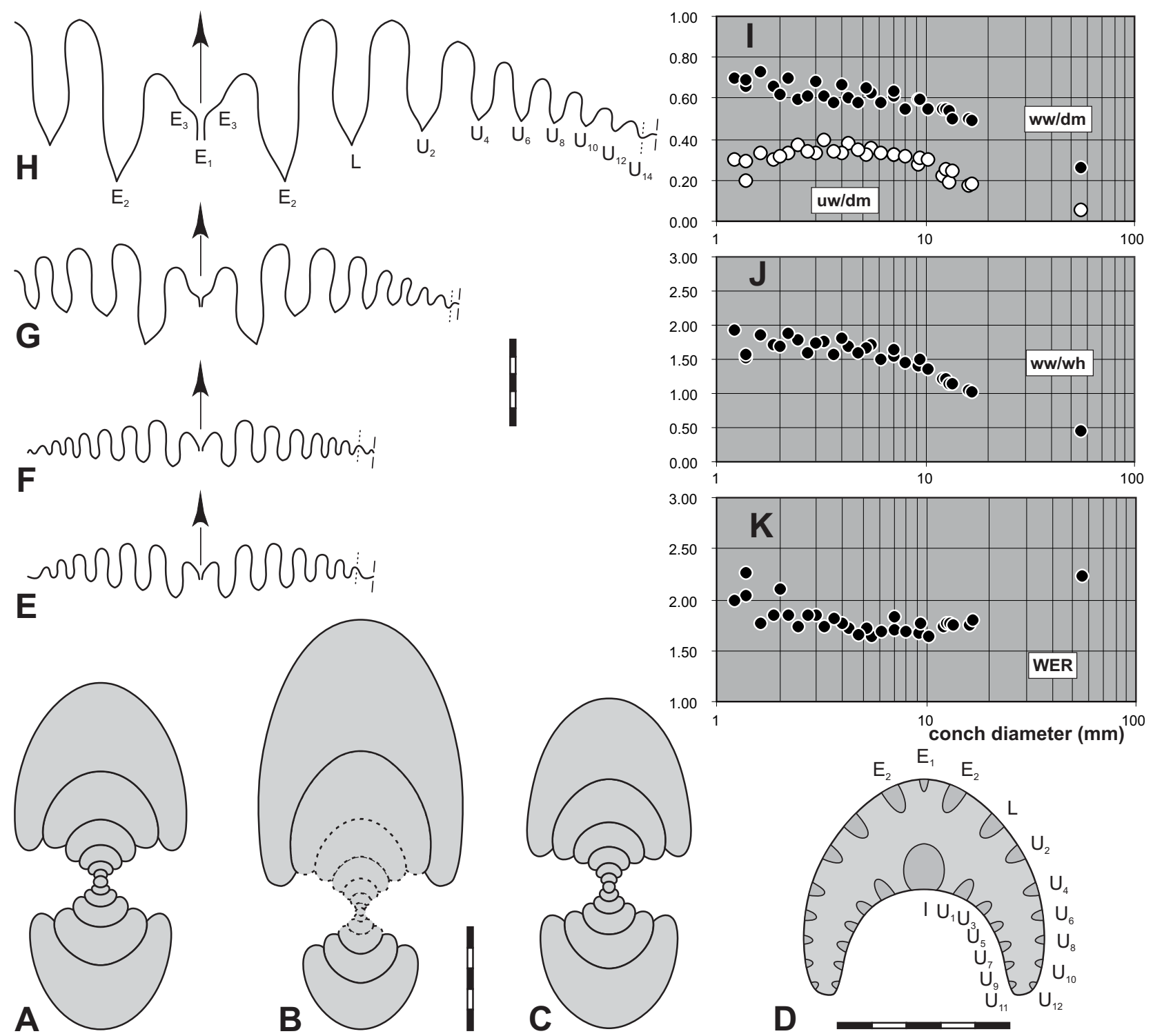

Figure 47. Meropharciceras disciforme (Bensaïd, 1974), topotypes from Hassi Nebech; A-C. Cross-sections, all $\times 3$; A. MB.C.22129.1; B. MB.C.22129.2; C. MB.C.22129.3; D. Septal face of MB.C.22129.4, $\times 5$; E-H. Sutures, all $\times 2.5$; E. MB.C. 22129.1 at $2.5 \mathrm{~mm}$ wh; F. MB.C. 22129.3 at $2.5 \mathrm{~mm}$ wh; G. MB.C. 22129.2 at $9.5 \mathrm{~mm}$ wh; H. MB.C.22129.5 at $12.5 \mathrm{~mm}$ wh; I-K. Ontogenetic development of ww/dm, uw/dm, ww/wh, and WER of all available specimens. 

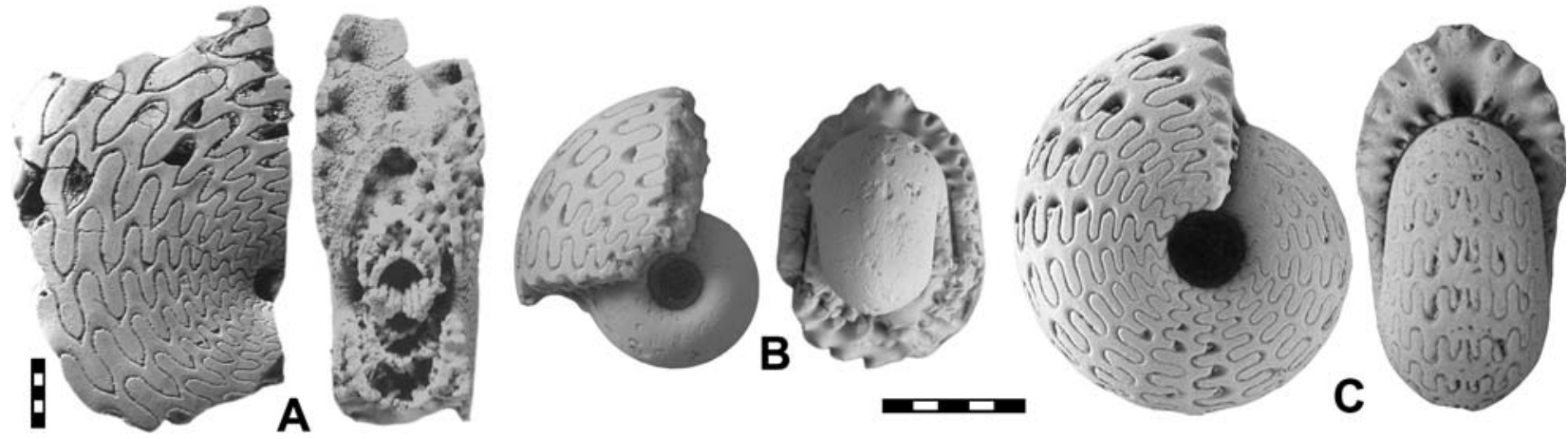

Figure 48. Meropharciceras disciforme (Bensaïd, 1974), topotypes from Hassi Nebech; A. MB.C.22129.5, × 1.5; B. MB.C. $22129.6, \times 3$; C. MB.C. $22129.4, \times 3$.

narrow $E_{1}$ lobe, incipient $E_{3}$ lobe forming a terrace on the diverging late part of the $E_{1}$, low median saddle, very deep, lanceolate $E_{2}$ lobe, very narrow and high lateral saddles, separated by a deep, lanceolate L lobe, and with a maximum of eight lateral U lobes, becoming less lanceolate and smaller towards the umbilicus, separated by gradually less high and less narrow saddles. Suture formula: $\left(E_{2}\left(E_{3}\right) E_{1}\left(E_{3}\right) E_{2}\right)$ $\mathrm{LU}_{2} \mathrm{U}_{4} \mathrm{U}_{6} \mathrm{U}_{8} \mathrm{U}_{10} \mathrm{U}_{12} \mathrm{U}_{14} \mathrm{U}_{16}: \mathrm{U}_{15} \mathrm{U}_{13} \mathrm{U}_{11} \mathrm{U}_{9} \mathrm{U}_{7} \mathrm{U}_{5} \mathrm{U}_{3} \mathrm{U}_{1} \mathrm{I}$.

\section{Synpharciceras Schindewolf, 1940}

Type species. Goniatites clavilobus Sandberger \& Sandberger, 1850.

\section{Synpharciceras sp.}

Figures 49, 50

Material. One specimen (MB.C.22123).

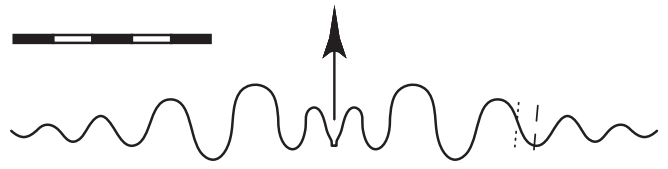

Figure 49. Suture of Stenopharciceras. sp. from Hassi Nebech, MB.C. 22123 at $5.6 \mathrm{~mm}$ wh, $\times 4$.
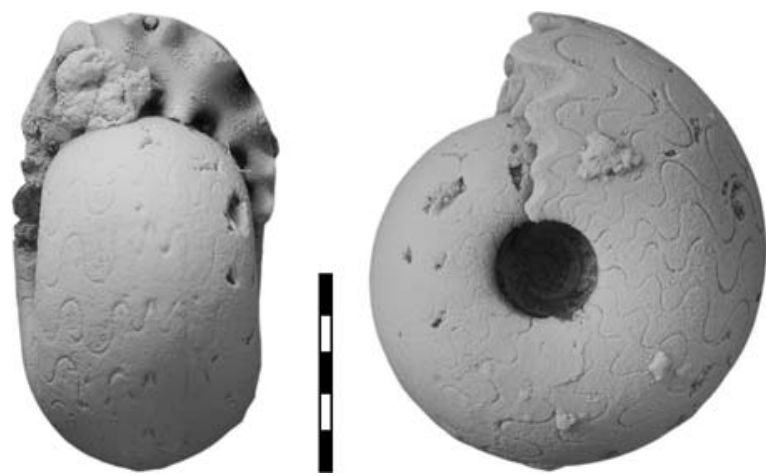

Figure 50. Stenopharciceras. sp. from Hassi Nebech, MB.C. $22123, \times 4$.

Table 46. Suture formula (Fig. 49), conch characteristics, and ornament of Synpharciceras sp.

$\left(E_{2} E_{1} E_{2}\right) L U_{2} U_{4} U_{6}: U_{5} U_{3} U_{1} l$ at $13 \mathrm{~mm} d m$

$E_{1}$ lobe funnel-shaped, as deep as $E_{2}$ lobe; ventrolateral saddle broadly rounded, symmetric

no ribs; no furrows; no mould constrictions

venter rounded, umbilicus narrow

growth lines not visible 


\section{Synpharciceras clavilobum (Sandberger \& Sandberger, 1850)}

Figures 51, 52

Type, Type locality, level, and diagnosis. See Bockwinkel et al. (2009).

Material. Ca. 50 specimens up to $40 \mathrm{~mm}$ conch diameter, including MB.C.22130.1-MB.C.22130.18.

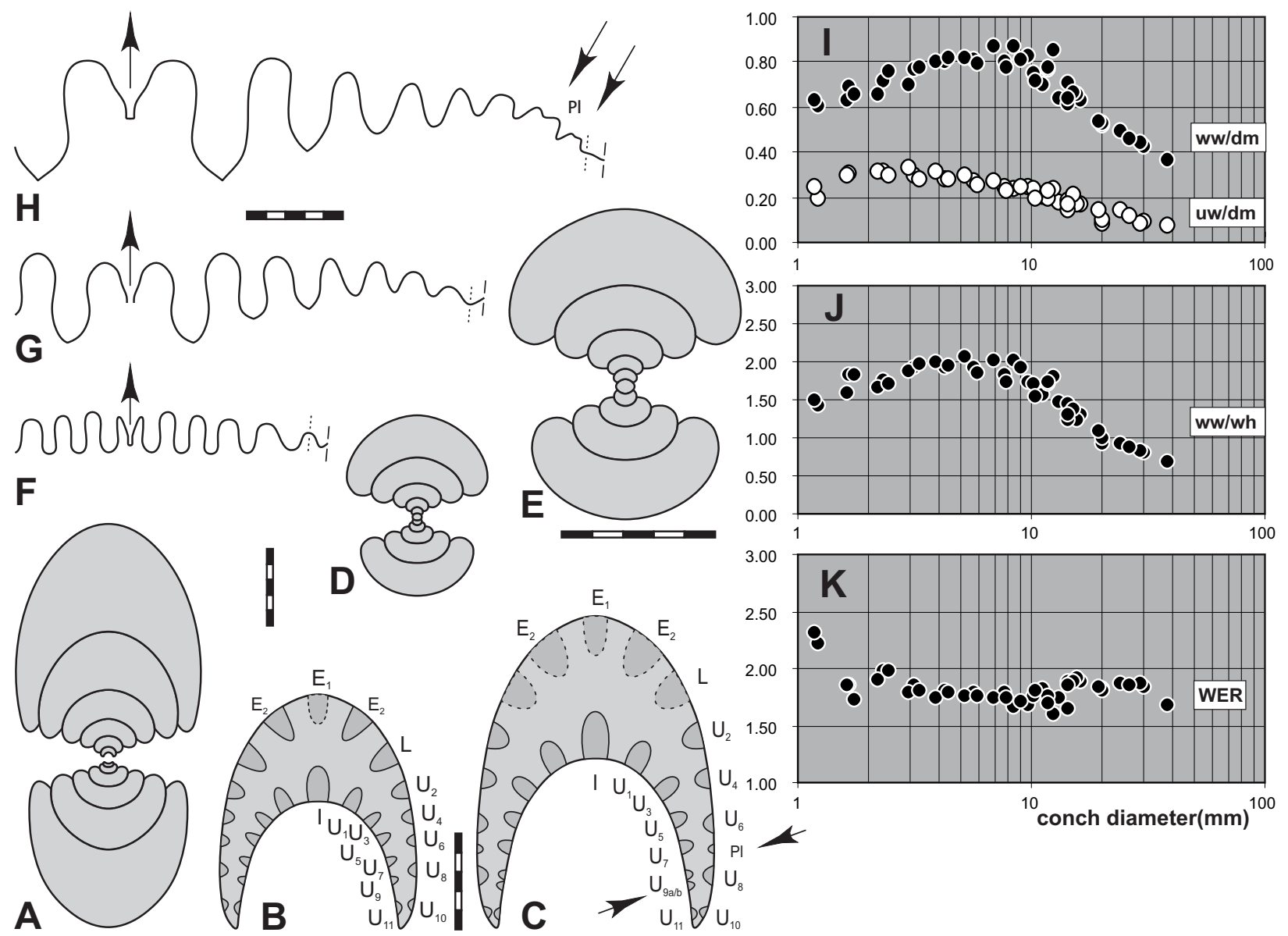

Figure 51. Synpharciceras clavilobum (Sandberger \& Sandberger, 1850) from Hassi Nebech; A. Cross-section of MB.C.22130.1, $\times 2$; B. Septal face of MB.C.22130.2, $\times 2.5$; C. Septal face of MB.C.22130.3, $\times 2.5$; D. Cross-section of MB.C.22130.4, $\times 2$; E. Cross-section of MB.C.22130.5, $\times$ 4; F. Suture of MB.C.22130.6 at $4.3 \mathrm{~mm}$ wh, $\times 2.5$; G. Suture of MB.C.22130.7 at $15 \mathrm{~mm}$ wh, $\times 2.5$; H. Suture of MB.C.22130.8 at $20.5 \mathrm{~mm}$ wh, $\times 2.5$; I-K. Ontogenetic development of ww $/ \mathrm{dm}$, uw $/ \mathrm{dm}$, ww/wh, and WER.

Table 47. Conch ontogeny (Figs 51A-E, I-K) of Synphaciceras clavilobum (Sandberger \& Sandberger, 1850) from Hassi Nebech.

\begin{tabular}{|c|c|c|c|}
\hline$d m$ & conch shape & whorl cross-section shape & whorl expansion \\
\hline $2 \mathrm{~mm}$ & $\begin{array}{l}\text { thinly pachyconic; subevolute } \\
\text { (ww/dm }=0.65-0.70 ; \text { uw/dm } \sim 0.30 \text { ) }\end{array}$ & $\begin{array}{l}\text { moderately depressed; moderately embracing } \\
\text { (ww/wh } \sim 1.70 ; \text { IZR } \sim 0.30 \text { ) }\end{array}$ & $\begin{array}{l}\text { moderate } \\
(\text { WER } \sim 1.90-2.00)\end{array}$ \\
\hline $7 \mathrm{~mm}$ & $\begin{array}{l}\text { thickly pachyconic; subinvolute } \\
\text { (ww/dm }=0.80-0.85 ; \text { uw/dm } \sim 0.25 \text { ) }\end{array}$ & $\begin{array}{l}\text { moderately depressed; strongly embracing } \\
\text { (ww/wh }=1.80-2.00 ; \text { IZR } \sim 0.40 \text { ) }\end{array}$ & $\begin{array}{l}\text { moderate } \\
(\text { WER } \sim 1.80)\end{array}$ \\
\hline $10 \mathrm{~mm}$ & $\begin{array}{l}\text { thickly pachyconic; subinvolute } \\
\text { (ww/dm }=0.70-0.80 ; \text { uw/dm } \sim 0.25 \text { ) }\end{array}$ & $\begin{array}{l}\text { moderately depressed; strongly embracing } \\
\text { (ww/wh } \sim 1.70 ; \text { IZR } \sim 0.45 \text { ) }\end{array}$ & $\begin{array}{l}\text { low } \\
(\text { WER } \sim 1.75)\end{array}$ \\
\hline $20 \mathrm{~mm}$ & $\begin{array}{l}\text { thickly discoidal; involute } \\
\text { (ww/dm } \sim 0.55 ; \text { uw/dm }=0.10-0.15 \text { ) }\end{array}$ & $\begin{array}{l}\text { weakly compressed; very strongly embracing } \\
\text { (ww/wh }=0.90-1.00 ; \text { IZR } \sim 0.50 \text { ) }\end{array}$ & $\begin{array}{l}\text { moderate } \\
(\text { WER } \sim 1.80)\end{array}$ \\
\hline 38 mm & $\begin{array}{l}\text { thinly discoidal; involute } \\
\text { (ww/dm } \sim 0.37 ; \text { uw/dm } \sim 0.08 \text { ) }\end{array}$ & $\begin{array}{l}\text { weakly compressed; very strongly embracing } \\
\text { (ww/wh } \sim 0.70 ; \text { IZR } \sim 0.57 \text { ) }\end{array}$ & $\begin{array}{l}\text { moderate } \\
(\mathrm{WER} \sim 1.70)\end{array}$ \\
\hline
\end{tabular}


Table 48. Suture formula (Figs 51B, C, F-H), conch characteristics, and ornament of Synphaciceras clavilobum (Sandberger \& Sandberger, 1850) from Hassi Nebech.

$\left(E_{2} E_{1} E_{2}\right) L U_{2} U_{4} U_{6}: U_{5} U_{3} U_{1} l$ at $8 \mathrm{~mm}$ dm (MB.C.22130.12)

$\left(E_{2} E_{1} E_{2}\right) L U_{2} U_{4} U_{6}: U_{7} U_{5} U_{3} U_{1} l$ at $12 \mathrm{~mm}$ dm (MB.C.22130.11)

$\left(\mathrm{E}_{2} \mathrm{E}_{1} \mathrm{E}_{2}\right) \mathrm{LU}_{2} \mathrm{U}_{4} \mathrm{U}_{6} \mathrm{U}_{8}: \mathrm{U}_{9} \mathrm{U}_{7} \mathrm{U}_{5} \mathrm{U}_{3} \mathrm{U}_{1} \mathrm{I}$ at $15 \mathrm{~mm} \mathrm{dm}$ (MB.C.22130.14)

$\left(E_{2} E_{1} E_{2}\right) L_{2} U_{4} U_{6} P_{I} U_{8} U_{10}: U_{11} U_{9 a b} U_{7} U_{5} U_{3} U_{1} l$ at $30 \mathrm{~mm} \mathrm{dm}$ (MB.C.22130.3)

$\left(E_{2} E_{1} E_{2}\right) L U_{2} U_{4} U_{6} P_{I U} P_{8} U_{10}: U_{11} U_{9} U_{7} U_{5} U_{3} U_{1}$ at $37 \mathrm{~mm} \mathrm{dm}$ (MB.C.22130.8)

plurilobation by subdivision of $U$ saddles; subdivision of inner $U$ lobes rare (MB.C.22130.3)

umbilicus narrow throughout ontogeny, no whorl overlap

no ribs; no mould constrictions; no furrows

growth lines biconvex, with moderately high ventrolateral salient

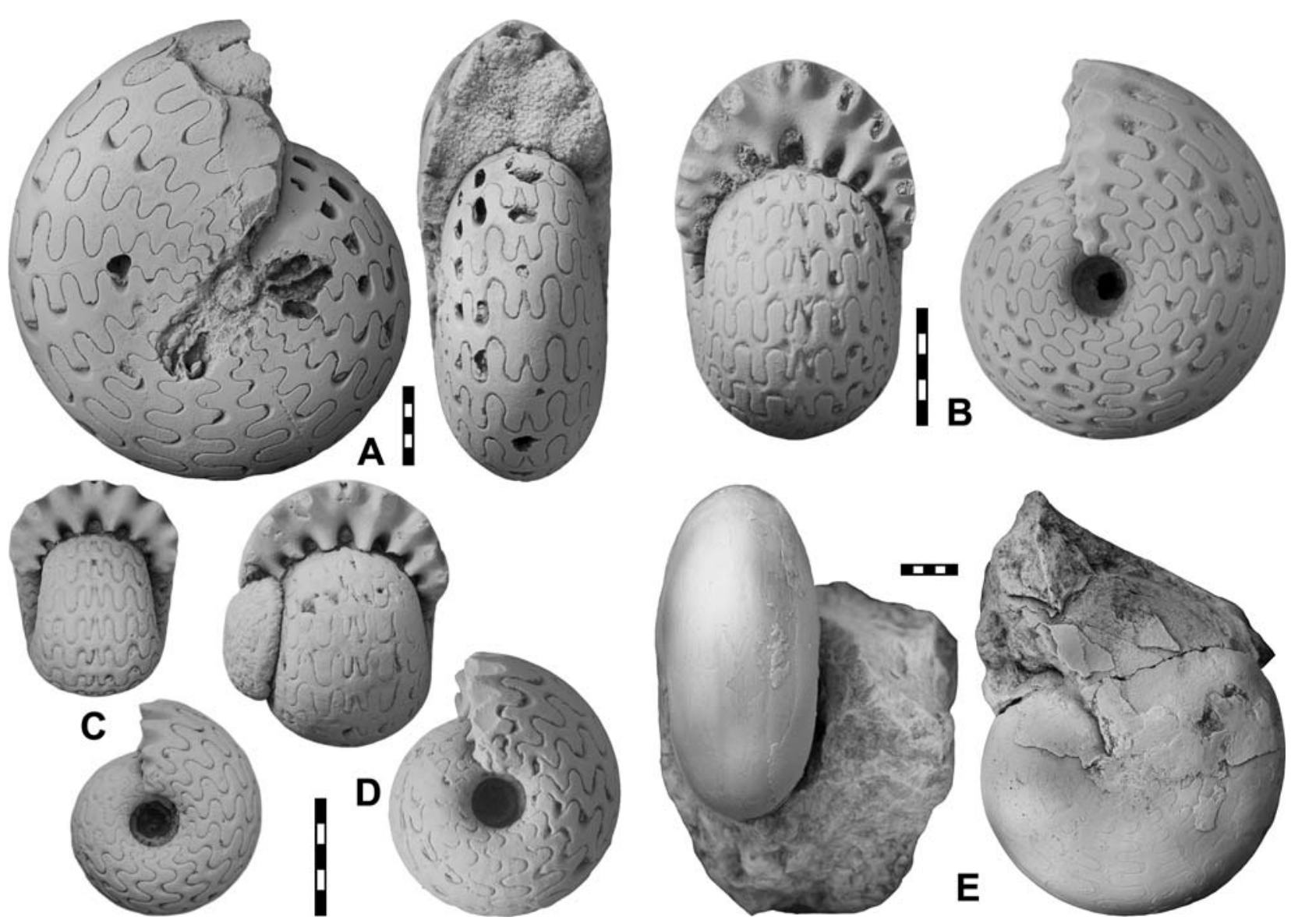

Figure 52. Synpharciceras clavilobum (Sandberger \& Sandberger, 1850) from Hassi Nebech; A. MB.C.22130.7, $\times 2$; B. MB.C. $22130.9, \times 3$; C. MB.C.22130.18, $\times 3$; D. MB.C.22130.11, $\times 3$; E. Reillustration of the holotype (original of Sandberger \& Sandberger 1850, pl. 8, figs 3, 3a, b), ×1.5. Museum Wiesbaden, Geology, Inv. No. 42.

\section{Synpharciceras frequens n. sp.}

Figures 53, 54

Derivation of name. Due to the frequent occurrence at Hassi Nebech.

Holotype. MB.C.22131.1 (Fig. 54B).

Type locality and horizon. Hassi Nebech, Section 2, SE Tafilalt, main collecting level, probably Taouzites taouzensis Zone (late Givetian).

Material. More than 250 specimens up to $40 \mathrm{~mm}$ conch diameter, including MB.C.22131.1-MB.C.22131.27.

Diagnosis. First $2 \frac{1}{2}$ whorls, up to ca. $3 \mathrm{~mm} \mathrm{dm}$, widely umbilicate, increasingly evolute (uw/dm rising to 0.42 ) and with ww/dm near 0.60 ; subsequent two whorls up to ca. $7 \mathrm{~mm} \mathrm{dm}$ smooth, slightly evolute (uw/dm up to 0.45 ), with depressed whorls (ww/wh up to 2.50 ) and with low WER (down to ca. 1.50); median stages until ca. $25 \mathrm{~mm}$ dm gradually less depressed to compressed (from $20 \mathrm{~mm} \mathrm{dm}$ ) and with WER rising to 1.70-1.80; the umbilicus closes rapidly, during the 6th whorl by absolute overlap of whorls; mature stage thinly discoidal, with narrow umbilicus (probably closed by umbilical shell flares), and with decreasing WER. Growth lines fine, sometimes bundled, strongly arched over 


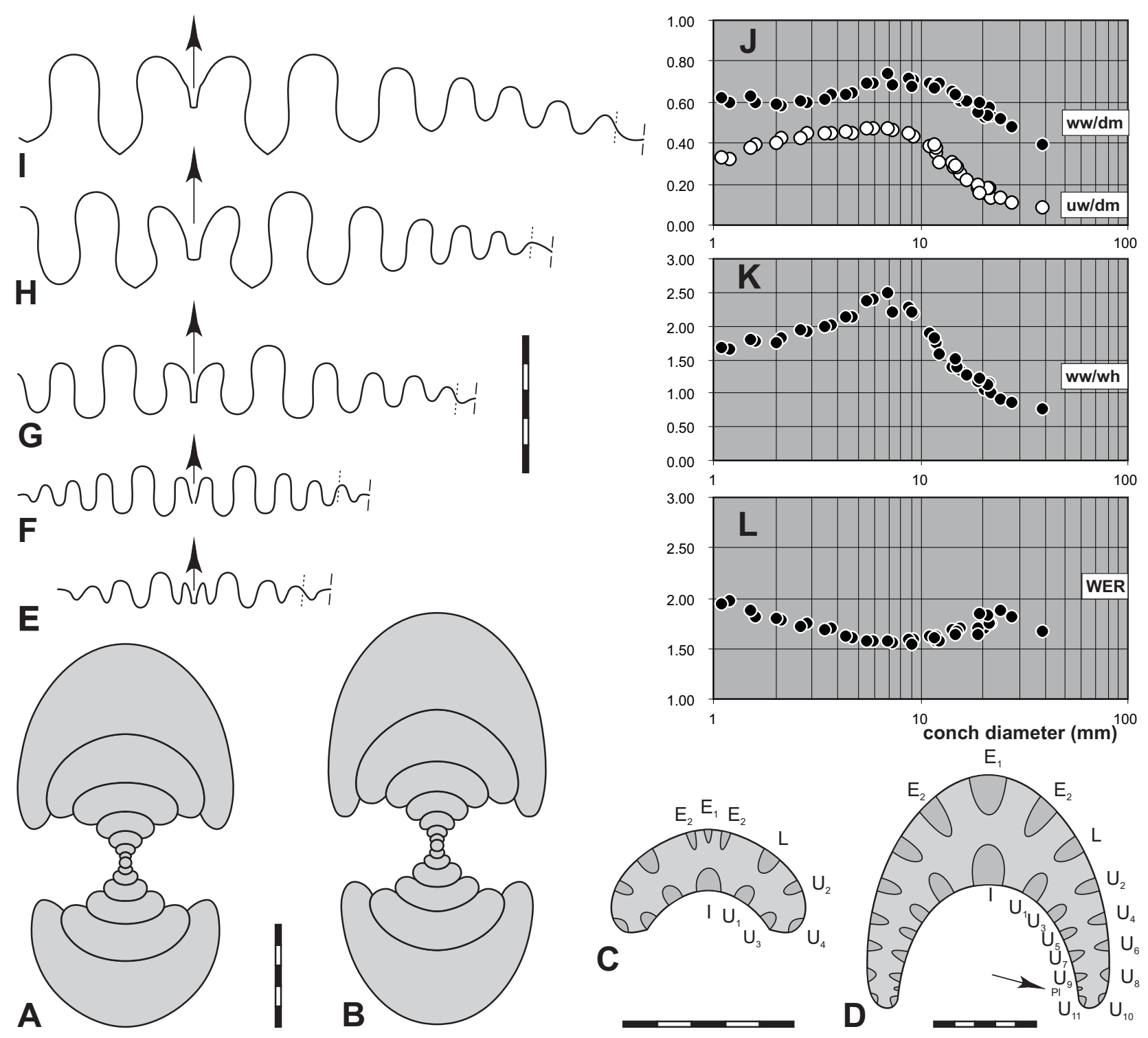

Figure 53. Synpharciceras frequens n. sp. from Hassi Nebech; A. Cross-section of paratype MB.C.22131.2, $\times 3$; B. Cross-section of paratype MB.C.22131.3, ×3; C. Septal face of MB.C.22131.4, $\times 5$; D. Septal face of paratype MB.C.22131.5, $\times 3$; E-I. Sutures, all $\times$ 4; E. MB.C.22131.4 at $2.5 \mathrm{~mm}$ wh; F. Paratype MB.C.22131.6 at $3.4 \mathrm{~mm}$ wh; G. Paratype MB.C.22131.7 at $8 \mathrm{~mm}$ wh; H. Paratype MB.C.22131.8 at $8.8 \mathrm{~mm}$ wh; I. Holotype MB.C.22131.1 at 13,6 mm wh, $\times 2.5$; J-L. Ontogenetic development of ww/dm, uw/dm, ww/wh, and WER.

the flanks. Mature sutures with divergent, funnel-shaped, short $\mathrm{E}_{1}$ lobe, moderately high median saddle, deep, lanceolate $\mathrm{E}_{2}$ lobe, high and narrow $\mathrm{E}_{2} \mathrm{~L}$ saddle, moderately deep, lanceolate $\mathrm{L}$ lobe, six, gradually smaller, shorter or wide and shallow $\mathrm{U}$ lobes, six internal $\mathrm{U}$ lobes, and deep, narrow I lobe. Suture formula: $\left(E_{2} E_{1} E_{2}\right) L_{2} U_{4} U_{6} U_{8} U_{10} U_{12}: U_{11} U_{9} U_{7} U_{5} U_{3} U_{1} I$.

Table 49. Conch ontogeny (Figs 53A-D, J-L) of Synphaciceras frequens $\mathrm{n}$. $\mathrm{sp}$.

\begin{tabular}{llll}
\hline $\mathrm{dm}$ & conch shape & whorl cross-section shape & whorl expansion \\
\hline $2 \mathrm{~mm}$ & thickly discoidal; subevolute & moderately depressed; moderately embracing & moderate \\
& $($ ww/dm $\sim 0.60 ;$ uw/dm $\sim 0.40)$ & (ww/wh $\sim 1.80 ;$ IZR $\sim 0.25)$ & $($ WER $\sim 1.80)$ \\
$7 \mathrm{~mm}$ & thinly pachyconic; evolute & strongly depressed; moderately embracing & low \\
& $($ ww/dm $\sim 0.70 ;$ uw/dm $\sim 0.45)$ & $($ ww/wh $=2.40-2.50 ;$ IZR $\sim 0.30)$ & $($ WER $\sim 1.60)$ \\
$10 \mathrm{~mm}$ & thinly pachyconic; subevolute & moderately depressed; strongly embracing & low \\
& $($ ww/dm $\sim 0.70 ;$ uw/dm $\sim 0.40)$ & $($ ww/wh $\sim 2.00 ;$ IZR $\sim 0.40)$ & $($ WER $\sim 1.60)$ \\
$20 \mathrm{~mm}$ & thickly discoidal; subinvolute & weakly depressed; very strongly embracing & moderate \\
& $($ ww/dm $\sim 0.55 ;$ uw/dm $=0.15-0.20)$ & $($ ww/wh $\sim 1.10 ;$ IZR $\sim 0.55)$ & $($ WER $=1.70-1.75)$ \\
\hline
\end{tabular}


Table 50. Suture formula (Figs 53C-I), conch characteristics, and ornament of Synphaciceras frequens n. sp.

\section{$\left(E_{2} E_{1} E_{2}\right) L U_{2} U_{4}: U_{5} U_{3} U_{1} I$ at $9 \mathrm{~mm}$ dm (MB.C.22131.27)}

$\left(E_{2} E_{1} E_{2}\right) L U_{2} U_{4} U_{6}: U_{7} U_{5} U_{3} U_{1} I$ at $12 \mathrm{~mm}$ dm (MB.C.22131.19)

$\left(E_{2} E_{1} E_{2}\right) L U_{2} U_{4} U_{6} U_{8}: U_{7} U_{5} U_{3} U_{1} l$ at $15 \mathrm{~mm}$ dm (MB.C.22131.14)

$\left(E_{2} E_{1} E_{2}\right) L U_{2} U_{4} U_{6} U_{8} U_{10}: U_{11} U_{9} U_{7} U_{5} U_{3} U_{1} l$ at $25 \mathrm{~mm}$ dm (holotype)

$\left(E_{2} E_{1} E_{2}\right) L U_{2} U_{4} U_{6} U_{8} U_{10}: U_{11} P_{I} U_{9} U_{7} U_{5} U_{3} U_{1} I$ at $22 \mathrm{~mm}$ dm (MB.C.22131.5, MB.C.22131.23)

$\left(E_{2} E_{1} E_{2}\right) L U_{2} U_{4} U_{6} U_{8} U_{10} U_{12}: U_{11} U_{9} U_{7} U_{5} U_{3} U_{1} l$ at $33 \mathrm{~mm} \mathrm{dm}$ (MB.C.22131.17)

plurilobation by subdivision of $U$ saddles

early whorls evolute; conch depressed; umbilicus closing by whorl overlap

no ribs; no mould constrictions; no furrows

growth lines convex, without ventrolateral salient

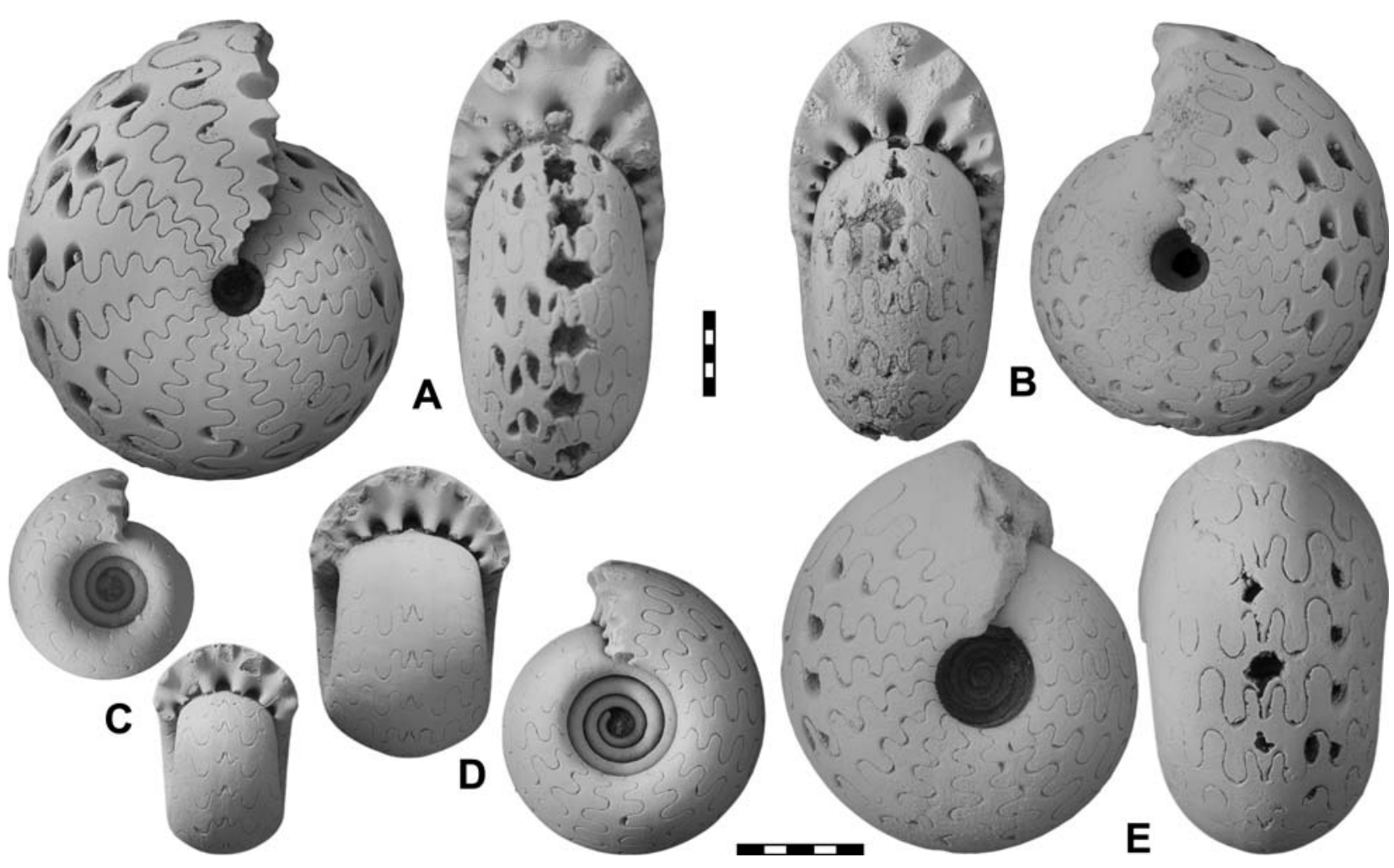

Figure 54. Synpharciceras frequens n. sp. from Hassi Nebech A. Paratype MB.C.22131.11, $\times 2$; B. Holotype MB.C.22131.1, $\times 2$; C. MB.C.22131.4, ×3; D. Paratype MB.C.22131.12, ×3; E. Paratype MB.C.22131.10, $\times 3$.

\section{Synpharciceras aff. frequens n. sp.}

\section{Figures 55}

Material. Three sectioned specimens (MB.C.22132.1-MB.C.22132.3).

Table 51. Conch ontogeny (Figs 55A-C, F-H) of Synphaciceras aff. frequens $\mathrm{n}$. $\mathrm{sp}$.

\begin{tabular}{|c|c|c|c|}
\hline $\mathrm{dm}$ & conch shape & whorl cross-section shape & whorl expansion \\
\hline $2 \mathrm{~mm}$ & $\begin{array}{l}\text { thickly pachyconic; subinvolute } \\
\text { (ww/dm } \sim 0.75 ; \text { uw/dm } \sim 0.30 \text { ) }\end{array}$ & $\begin{array}{l}\text { moderately depressed; strongly embracing } \\
\text { (ww/wh } \sim 1.80 \text {; IZR } \sim 0.35 \text { ) }\end{array}$ & $\begin{array}{l}\text { moderate } \\
(\text { WER } \sim 1.85)\end{array}$ \\
\hline $7 \mathrm{~mm}$ & $\begin{array}{l}\text { thickly pachyconic; subevolute } \\
\text { (ww/dm } \sim 0.80 ; \text { uw/dm } \sim 0.40 \text { ) }\end{array}$ & $\begin{array}{l}\text { strongly depressed; strongly embracing } \\
\text { (ww/wh } \sim 2.35 \mathrm{IZR} \sim 0.40 \text { ) }\end{array}$ & $\begin{array}{l}\text { low } \\
\text { (WER 1.60) }\end{array}$ \\
\hline $10 \mathrm{~mm}$ & $\begin{array}{l}\text { thickly pachyconic; subevolute } \\
\text { (ww/dm } \sim 0.80 ; \text { uw/dm } \sim 0.40 \text { ) }\end{array}$ & $\begin{array}{l}\text { strongly depressed; strongly embracing } \\
(\text { ww/wh } \sim 2.30 ; \text { IZR } \sim 0.40)\end{array}$ & $\begin{array}{l}\text { low } \\
(\text { WER } \sim 1,55)\end{array}$ \\
\hline $20 \mathrm{~mm}$ & $\begin{array}{l}\text { thickly discoidal; involute } \\
\text { (ww/dm } \sim 0.60 ; \text { uw/dm } \sim 0.15 \text { ) }\end{array}$ & $\begin{array}{l}\text { weakly depressed; very strongly embracing } \\
\text { (ww/wh } \sim 1.20 \text {; IZR } \sim 0.50 \text { ) }\end{array}$ & $\begin{array}{l}\text { low } \\
\text { (WER 1,70) }\end{array}$ \\
\hline
\end{tabular}


Table 52. Suture formula (Figs 55D, E), conch characteristics, and ornament of Synphaciceras aff. frequens n. sp.

$\left(E_{2} E_{1} E_{2}\right) L U_{2} U_{4} U_{6}: \ldots \ldots \ldots \ldots \ldots$ at $12 \mathrm{~mm} \mathrm{dm} \mathrm{(MB.C.22132.2)}$
$\left(E_{2} E_{1} E_{2}\right) L U_{2} U_{4} U_{6} U_{8}: \ldots \ldots \ldots \ldots \ldots$ at $15 \mathrm{~mm} \mathrm{dm} \mathrm{(MB.C.22132.3)}$
$\left(E_{2} E_{1} E_{2}\right) L U_{2} U_{4} U_{6} U_{8} U_{10}: \ldots \ldots \ldots \ldots \ldots$ at $20 \mathrm{~mm} \mathrm{dm} \mathrm{(MB.C.22132.1)}$
$E_{1}$ lobe short, narrow; flank saddles self-similar
venter broadly rounded, involute
no ribs; no constrictions; no furrows
growth lines not visible
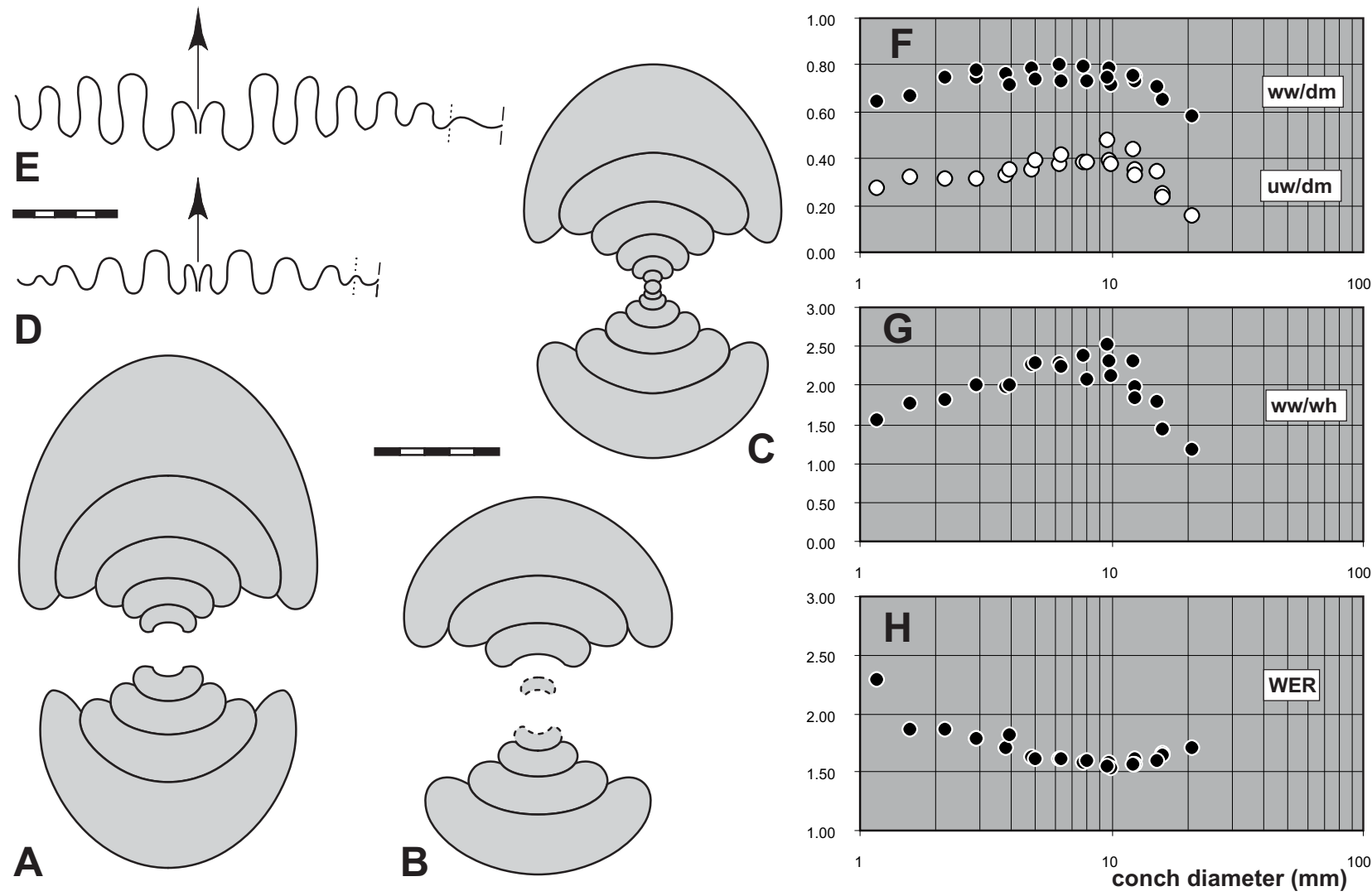

Figure 55. Synpharciceras aff. frequens n. sp. from Hassi Nebech; A. Cross-section of MB.C.22132.1, $\times 3$; B. Cross-section of MB.C.22132.2, $\times 3$; C. Cross-section of MB.C.22132.3, $\times 3$; D. Suture of MB.C.22132.2 at $5 \mathrm{~mm}$ wh, $\times 2.5$; E. Suture of MB.C.22132.1 at $10.5 \mathrm{~mm}$ wh, $\times 2.5 ; \mathbf{F}-\mathbf{H}$. Ontogenetic development of ww/dm, uw/dm, ww/wh, and WER of all available specimens.

\section{Family Petteroceratidae Becker \& House, 1993}

Diagnosis (emend). Moderate to large-sized, early whorls depressed, subinvolute to evolute, mature whorls compressed, subinvolute, with rounded or oxyconic venter. Sutures with incipient to fully developed third E lobe and six to thirteen U lobes, often with more outer than inner ones.

Discussion. Our new observations at Hassi Nebech specimens show that the U lobes do not develop in the regular, alternating way as in typical Pharciceratinae and Synpharciceratinae. Petteroceras specimens display more outer than inner U lobes, which was first noted in Petter (1959, fig. $33 \mathrm{H}_{2}$ ). As discussed under Extropharciceras n. sp., it is possible that Clariondites, despite its incipient $\mathrm{E}_{3}$ lobe, is not related to Petteroceras.

\section{Petteroceras Bogoslovsky, 1962}

Type species. Pharciceras? errans Petter, 1959.

Diagnosis. See Bogoslovsky (1962) or Bensaïd (1974). Suture formula: $\left(E_{2} E_{3} E_{1} E_{3} E_{2}\right) L_{U_{2}} U_{4} U_{5} U_{7} U_{9}: U_{8} U_{6} U_{3} U_{1} I$. 


\section{Petteroceras errans (Petter, 1959)}

Figures 56, 57

Lectotype. The original of Petter (1959, pl. 8, fig. 11) is here selected as lectotype and re-illustrated in Figure 57A (including for the first time a ventral view). It was first figured in Termier \& Termier (1950, pl. 151, figs 27-30) as Beloceras Denckmanni and is stored in the Musée d'Histoire Naturelle, Paris. It is a medium-sized fragment showing conch shape and outer and inner sutures.

Type locality and horizon. Kreb Oued Amerloh, Tafilalt; Petteroceras errans Zone.

Material. More than 30 specimens up to ca. $55 \mathrm{~mm}$ conch diameter, including MB.C.22133.1-MB.C.22133.19.

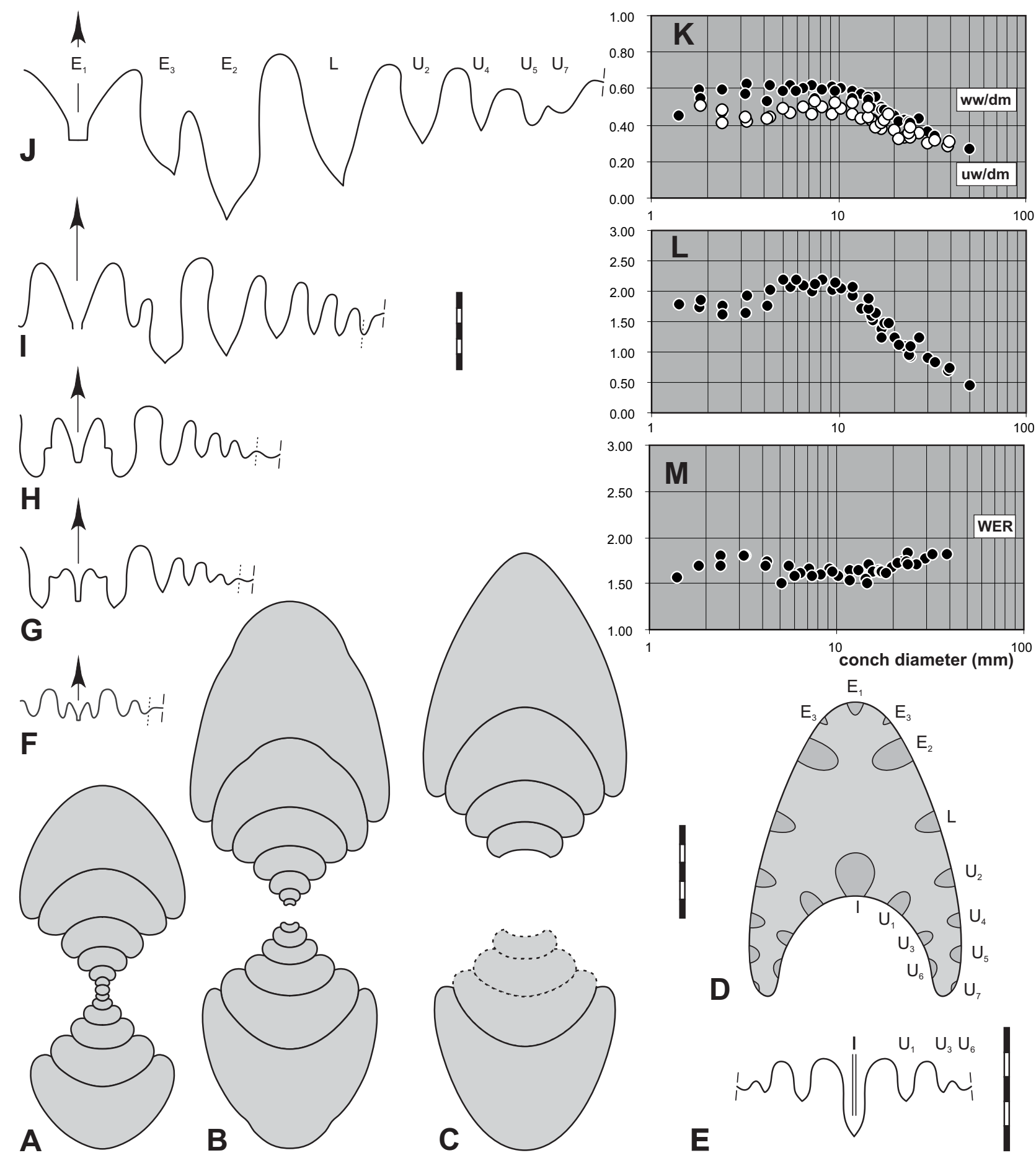

Figure 56. Petteroceras errans (Petter, 1959) from Hassi Nebech; A-C. Cross-sections, all $\times 3$; A. MB.C.22133.1; B. MB.C.22133.2; C. MB.C.22133.3; D. Septal face of MB.C.22133.4, $\times 3$; E. MB.C.22133.5, dorsal lobes at $9.5 \mathrm{~mm} w w, \times 4$; F-J. Sutures, all $\times 2.5$; F. MB.C.22133.6 at $2.8 \mathrm{~mm}$ wh; G. MB.C. 22133.7 at $8.8 \mathrm{~mm}$ wh; H. MB.C.22133.8 at $10 \mathrm{~mm}$ wh; I. MB.C.22133.9 at $16 \mathrm{~mm}$ wh; J. MB.C.22133.10 at $30 \mathrm{~mm}$ wh; K-M. Ontogenetic development of ww/dm, uw/dm, ww/ wh, and WER. 

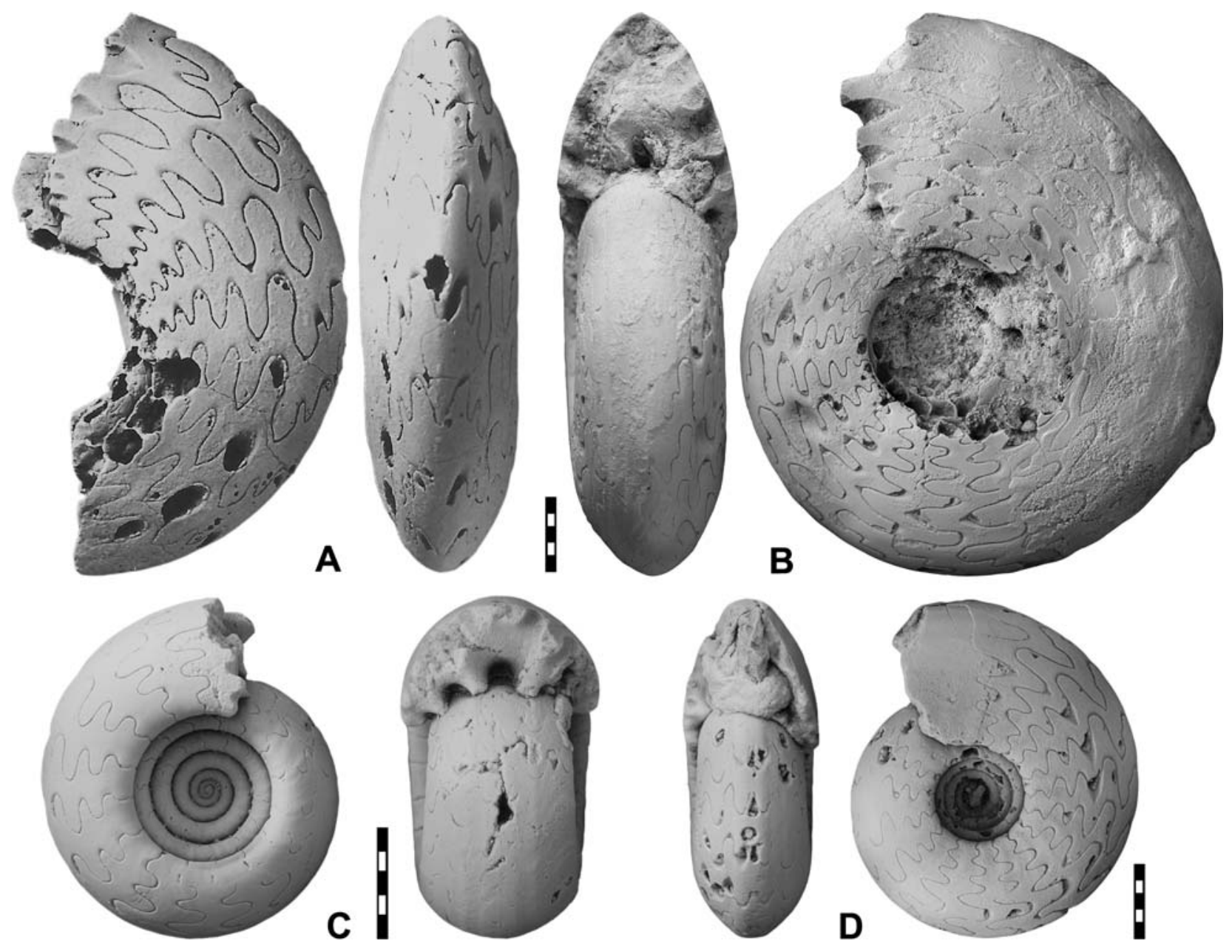

Figure 57. Petteroceras errans (Petter, 1959); A. Lectotype (designated here) from Kreb Oued Amerloh, original of Beloceras Denckmanni in Termier \& Termier (1950, pl. 151, figs 27-30) and of Ph. errans in Petter (1959, pl. 8, fig. 11), $\times 2$; B-D. Specimens from Hassi Nebech; B. MB.C.22133.9, $\times 2$; C. MB.C.22133.11, $\times 3$; D. MB.C.22133.12, $\times 2$.

Diagnosis (emend.). Post-embryonic conch ontogeny triphasic; first two whorls, until $3 \mathrm{~mm} \mathrm{dm}$, with decreasing umbilical width, rising whorl width (ww/dm up to 0.60 ) and WER values; between 3 and $10 \mathrm{~mm} \mathrm{dm}$ (until 5th whorl) broadly depressed, slowly expanding (WER 1.601.70), with constant ww/dm ratio, rising ww/wh (up to 2.00), and increasing umbilical width rate (uw/dm up to 0.50 ); submature and mature stages gradually less depressed to compressed (from ca. $25 \mathrm{~mm} \mathrm{dm}$ on), and finally thinly compressed and oxyconic (from $50 \mathrm{~mm} \mathrm{dm}$ on); the relative umbilical width decreases to below 0.30. Early stages with dense subumbilical ribbing. Growth lines strongly biconvex, with narrow ventrolateral salient lying in spiral (double) furrows. Mature sutures with divergent, short, funnel-shaped $\mathrm{E}_{1}$ lobe, deeper, asymmetrically inward flexured $\mathrm{E}_{3}$ lobe, very narrow, low inner median saddle, very deep, asymmetric lanceolate $\mathrm{E}_{2}$ lobe, very high and narrow ventrolateral saddle, asymmetric, pointed, moderately deep L lobe, two pointed and two rounded, small outer $\mathrm{U}$ lobes towards the umbilicus, separated by relative high and narrow saddles, with the exception of the smallest $U_{5} U_{7}$ saddle. Mature suture formula: $\left(E_{2} E_{3} E_{1} E_{3} E_{2}\right)$ $\mathrm{LU}_{2} \mathrm{U}_{4} \mathrm{U}_{5} \mathrm{U}_{7} \mathrm{U}_{9}: \mathrm{U}_{8} \mathrm{U}_{6} \mathrm{U}_{3} \mathrm{U}_{1} \mathrm{I}$

Table 53. Conch ontogeny (Figs 56A-D, K-M) of Petteroceras errans (Petter, 1959) from Hassi Nebech.

\begin{tabular}{|c|c|c|c|}
\hline$d m$ & conch shape & whorl cross-section shape & whorl expansion \\
\hline $2 \mathrm{~mm}$ & $\begin{array}{l}\text { thickly discoidal; subevolute to evolute } \\
(\mathrm{ww} / \mathrm{dm}=0.50-0.60 ; \mathrm{uw} / \mathrm{dm}=0.40-0.50)\end{array}$ & $\begin{array}{l}\text { moderately depressed; moderately embracing } \\
(\mathrm{ww} / \mathrm{wh}=1.70-1.80 ; \mathrm{IZR}=0.20-0.25)\end{array}$ & $\begin{array}{l}\text { low to moderate } \\
(\mathrm{WER}=1.70-1.80)\end{array}$ \\
\hline $8 \mathrm{~mm}$ & $\begin{array}{l}\text { thickly discoidal; evolute } \\
\text { (ww/dm } \sim 0.60 ; \text { uw/dm }=0.45-0.50 \text { ) }\end{array}$ & $\begin{array}{l}\text { strongly depressed; moderately embracing } \\
\text { (ww/wh }=2.00-2.20 ; \text { IZR }=0.25-0.30)\end{array}$ & $\begin{array}{l}\text { low } \\
(\text { WER }=1.60-1.70)\end{array}$ \\
\hline $20 \mathrm{~mm}$ & $\begin{array}{l}\text { thinly discoidal; subevolute } \\
\text { (ww/dm } \sim 0.45 ; \mathrm{uw} / \mathrm{dm}=0.35-0.40 \text { ) }\end{array}$ & $\begin{array}{l}\text { weakly depressed; strongly embracing } \\
(\mathrm{ww} / \mathrm{wh}=1.00-1.20 ; \mathrm{IZR}=0.35-0.40)\end{array}$ & $\begin{array}{l}\text { low to moderate } \\
(\mathrm{WER}=1.70-1.80)\end{array}$ \\
\hline $40 \mathrm{~mm}$ & $\begin{array}{l}\text { extremely discoidal; subinvolute } \\
\text { (ww/dm } \sim 0.30 ; \text { uw/dm } \sim 0.30 \text { ) }\end{array}$ & $\begin{array}{l}\text { weakly compressed; strongly embracing } \\
\text { (ww/wh } \sim 0.75 ; \text { IZR } \sim 0.40 \text { ) }\end{array}$ & $\begin{array}{l}\text { moderate } \\
(\text { WER } \sim 1.80)\end{array}$ \\
\hline
\end{tabular}


Table 54. Suture formula (Figs 56D-J), conch characteristics, and ornament of Petteroceras errans (Petter, 1959) from Hassi Nebech.

$\left(E_{2} E_{1} E_{2}\right) L U_{2} U_{4}: U_{3} U_{1} l$ at ca. $12 \mathrm{~mm} d m$ (MB.C.22133.6)

$\left(E_{2} E_{3} E_{1} E_{3} E_{2}\right) L U_{2} U_{4} U_{5}: U_{3} U_{1} l$ at ca. $15 \mathrm{~mm}$ dm (MB.C.22133.11)

$\left(\mathrm{E}_{2} \mathrm{E}_{3} \mathrm{E}_{1} \mathrm{E}_{3} \mathrm{E}_{2}\right) \mathrm{LU}_{2} \mathrm{U}_{4} \mathrm{U}_{5} \mathrm{U}_{7}: \mathrm{U}_{6} \mathrm{U}_{3} \mathrm{U}_{1} \mathrm{l}$ at $40 \mathrm{~mm}$ dm (MB.C.22133.9)

$\left(E_{2} E_{3} E_{1} E_{3} E_{2}\right) L U_{2} U_{4} U_{5} U_{7} U_{9}: U_{8} U_{6} U_{3} U_{1} l$ at ca. $55 \mathrm{~mm}$ dm (MB.C.22133.5)

$E_{1}$ lobe funnel-shaped, moderately deep; $E_{2}$ lobe deep and large, pointed; $E_{3}$ lobe small and steplike in juveniles, deeper and acute in adults; $L$ lobe shorter than $E_{2}$ lobe; all saddles narrowly rounded; $L$ and outer $U$ lobes pointed; asymmetrical development of the $U$ lobes from $U_{5}$ venter rounded at $20 \mathrm{~mm} \mathrm{dm}$, subacute at $35 \mathrm{~mm} \mathrm{dm}$, acute at $50 \mathrm{~mm} \mathrm{dm}$; umbilicus moderately wide

weak ribs in juveniles; strongly ventrolateral furrows (MB.C.22133.7) or double furrows (MB.C.22133.8) present or not (MB.C.22133.10); no mould constrictions

growth lines rectiradiate, concavo-convex with high ventrolateral projection and deep lateral sinus

Order Goniatitida Hyatt, 1884

Suborder Tornoceratina Wedekind, 1918

Superfamily Tornocerataceae von Arthaber, 1911

Family Tornoceratidae von Arthaber, 1911

Subfamily Tornoceratinae von Arthaber, 1911

Diagnosis (emend.). Conch in first post-embryonic whorl evolute, whorl cross-section depressed, subsequent whorls with completely closing umbilicus, whorl cross-section gradually weakly to strongly compressed, WER moderately high to high; venter narrowly rounded, oxyconic, or tabulate; growth lines biconvex to (secondarily) convex, ventrolateral furrows only in some derived forms; shell mostly smooth, rarely with ribs, with or without internal shell thickenings. L lobe undivided or divided, A lobe wide and shallow or deep, lingulate to asymmetrically pointed, external lobe small. Suture formulae: EALI to $\mathrm{EAL}_{\mathrm{e}}: \mathrm{L}_{\mathrm{i}} \mathrm{I}$

\section{Epitornoceras Frech, 1902}

Type species. Goniatites mithracoides Frech, 1888 (OD).

Diagnosis, included species, stratigraphic range and geographic distribution. See Bockwinkel et al. (2009).

\section{Epitornoceras mithracoides (Frech, 1888)}

Figures 4D, 58, 59

Lectotype. MB.C.469, designated in Bockwinkel et al. (2009).

Type locality and horizon. Volpertseiche Mine near Eibach, Dill Syncline, southern Rhenish Massive; late Givetian, precise horizon unknown.

Material. More than 15 specimens from the main collecting horizon, up to $27.5 \mathrm{~mm}$ conch diameter, including MB.C.22101.1-MB.C.22101.9; MB.C.22137 from the "Lower Marker Bed".

Diagnosis. See Bockwinkel et al. (2009).

Table 55. Conch ontogeny (Figs 58A-C, G-I) of Epitornoceras mithracoides (Frech, 1888) from Hassi Nebech.

\begin{tabular}{|c|c|c|c|}
\hline$d m$ & conch shape & whorl cross-section shape & whorl expansion \\
\hline $2 \mathrm{~mm}$ & $\begin{array}{l}\text { thickly discoidal; subinvolute } \\
\text { (ww/dm } \sim 0.60 ; \text { uw/dm } \sim 0.20 \text { ) }\end{array}$ & $\begin{array}{l}\text { weakly depressed; moderately embracing } \\
\text { (ww/wh }=1.40-1.50 ; \text { IZR } \sim 0.30 \text { ) }\end{array}$ & $\begin{array}{l}\text { moderate } \\
(\text { WER } \sim 2.00)\end{array}$ \\
\hline $5 \mathrm{~mm}$ & $\begin{array}{l}\text { thickly discoidal; involute } \\
\text { (ww/dm } \sim 0.50 ; \text { uw/dm } \sim 0.10 \text { ) }\end{array}$ & $\begin{array}{l}\text { weakly compressed; strongly embracing } \\
\text { (ww/wh }=0.90-1.00 ; \text { IZR } \sim 0.45 \text { ) }\end{array}$ & $\begin{array}{l}\text { high } \\
\text { (WER 2.15) }\end{array}$ \\
\hline $10 \mathrm{~mm}$ & $\begin{array}{l}\text { thinly discoidal; involute } \\
\text { (ww/dm } \sim 0.45 ; \text { uw/dm } \sim 0.02 \text { ) }\end{array}$ & $\begin{array}{l}\text { weakly compressed; strongly embracing } \\
\text { (ww/wh } \sim 0.80 ; \text { IZR } \sim 0.45 \text { ) }\end{array}$ & $\begin{array}{l}\text { high } \\
\text { (WER 2.15) }\end{array}$ \\
\hline $25 \mathrm{~mm}$ & $\begin{array}{l}\text { thinly discoidal; involute } \\
\text { (ww/dm } \sim 0.40 ; \text { uw/dm } \sim 0.01 \text { ) }\end{array}$ & $\begin{array}{l}\text { weakly compressed; strongly embracing } \\
\text { (ww/wh } \sim 0.65 ; \text { IZR } \sim 0.45 \text { ) }\end{array}$ & $\begin{array}{l}\text { high } \\
\text { (WER } \sim 2.20)\end{array}$ \\
\hline
\end{tabular}

Table 56. Suture formula (Figs 58B, D-F), conch characteristics, and ornament of Epitornoceras mithracoides (Frech, 1888) from Hassi Nebech.

EALI

E lobe deep, wide, funnel-shaped; A lobe deeply rounded, slightly asymmetric; ventrolateral saddle very narrow, high, subacute venter narrowly rounded in juveniles, nearly suboxyconic at $30 \mathrm{~mm} \mathrm{dm}$; umbilical ramp flat no furrows; no ribs; no internal constrictions

growth lines biconvex 

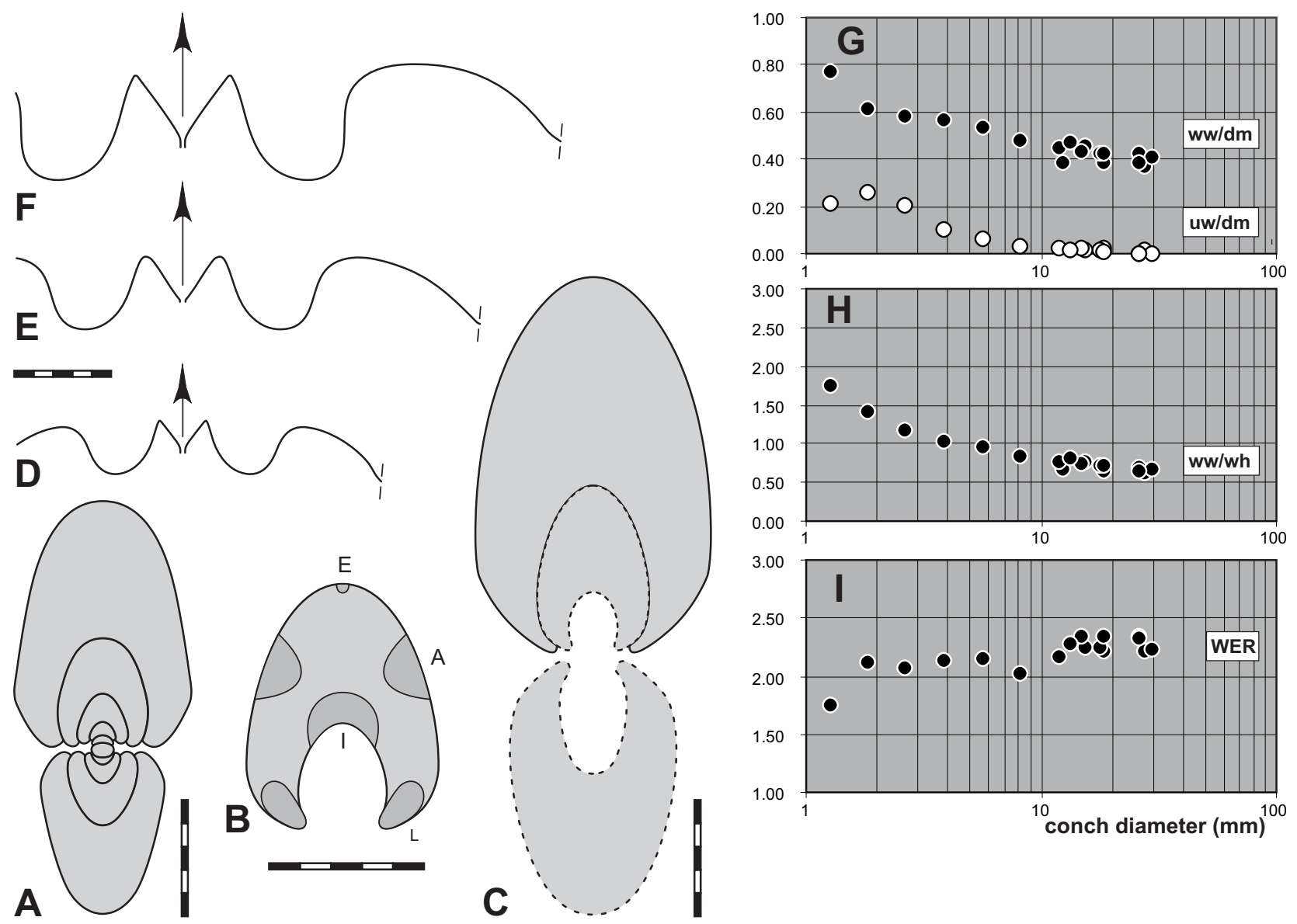

Figure 58. Epitornoceras mithracoides (Frech, 1902) from Hassi Nebech; A. Cross-section of MB.C.22101.1, $\times 3$; B. Septal face of MB.C.22101.2, $\times 4$; C. Cross-section of MB.C.22101.3, $\times 3$; D. Suture of MB.C.22101.4 at $7 \mathrm{~mm}$ wh, $\times 2.5$; E. Suture of MB.C.22101.5 at $11.5 \mathrm{~mm}$ wh, $\times 2.5$; F. Suture of MB.C.22101.5 at $16.5 \mathrm{~mm}$ wh, $\times 2.5$; G-I. Ontogenetic development of ww/dm, $\mathrm{uw} / \mathrm{dm}, \mathrm{ww} / \mathrm{wh}$, and WER.
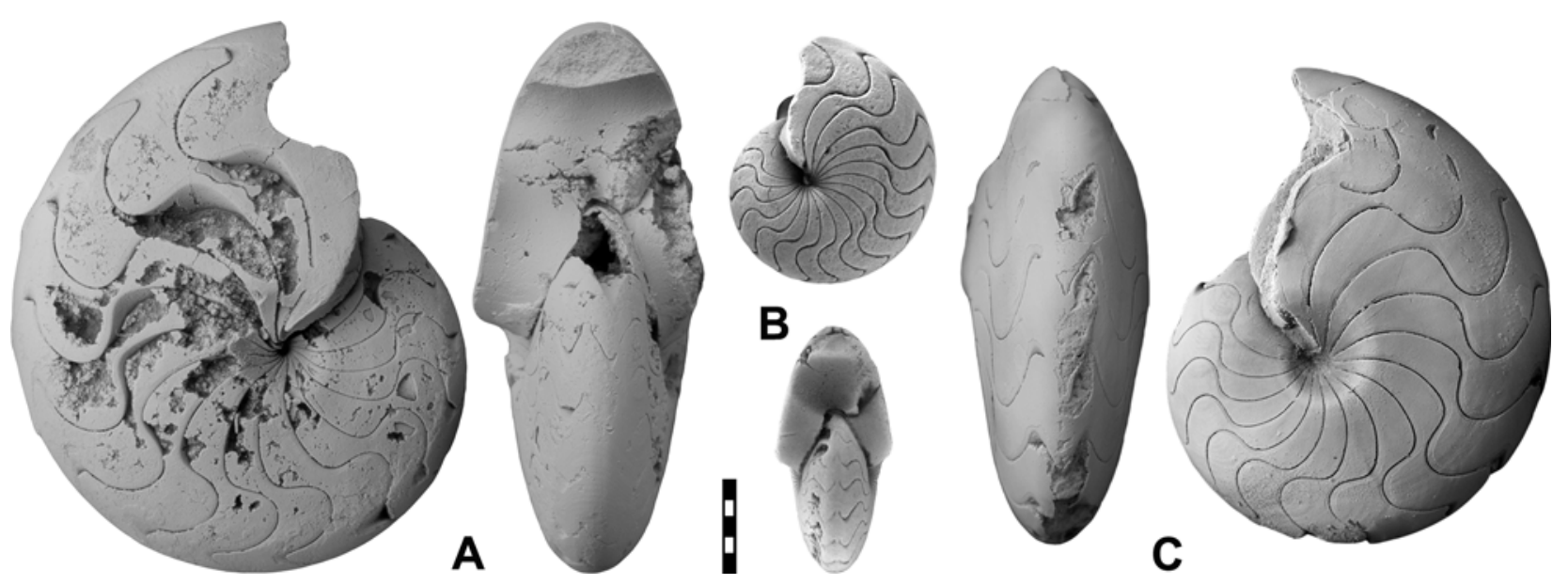

Figure 59. Epitornoceras mithracoides (Frech, 1902) from Hassi Nebech; A. MB.C.22101.5; B. MB.C.22101.2; C. MB.C.22101.6; all × 2 .

\section{Lobotornoceras Schindewolf, 1936}

Type species. Goniatites Ausavensis Steininger, 1849.

Diagnosis (emend.). Very small-sized, earliest whorls depressed, subsequent whorls involute or with closed umbilicus, compressed, thinly discoidal, with flattened flanks and narrowly rounded venter; WER high; growth lines slightly prorsiradiate, weakly biconvex, with shallow flank sinus and low ventrolateral projection; with or without mould constrictions, smooth, without spiral furrows; L lobe subdivided by small and low saddle at umbilical seam, lateral saddles moderately high, A lobe rounded, E lobe small. Suture formula: $\mathrm{EAL}_{\mathrm{e}}: \mathrm{L}_{\mathrm{i}} \mathrm{I}$ 


\section{Lobotornoceras bensaidi $\mathrm{n} . \mathbf{s p}$.}

Figures 4C, 60, 61

Derivation of name. In honour of Dr. M. Bensaïd (Rabat), who first illustrated the new species.

Holotype. MB.C.22102.1 (Fig. 61D).

Type locality and horizon. Hassi Nebech, Section 2, SE Tafilalt, main collecting level, probably Taouzites taouzensis Zone (late Givetian).

Material. Four specimens up to ca. $12.5 \mathrm{~mm}$ conch diameter, MB.C.22102.1-MB.C.22102.3, and paratype STIPB-Bensaïd-44 (old: GPI Bo 44), the original of Bensaïd (1974, pl. 5, figs 4, 4a, re-illustrated here in Figure 61A).
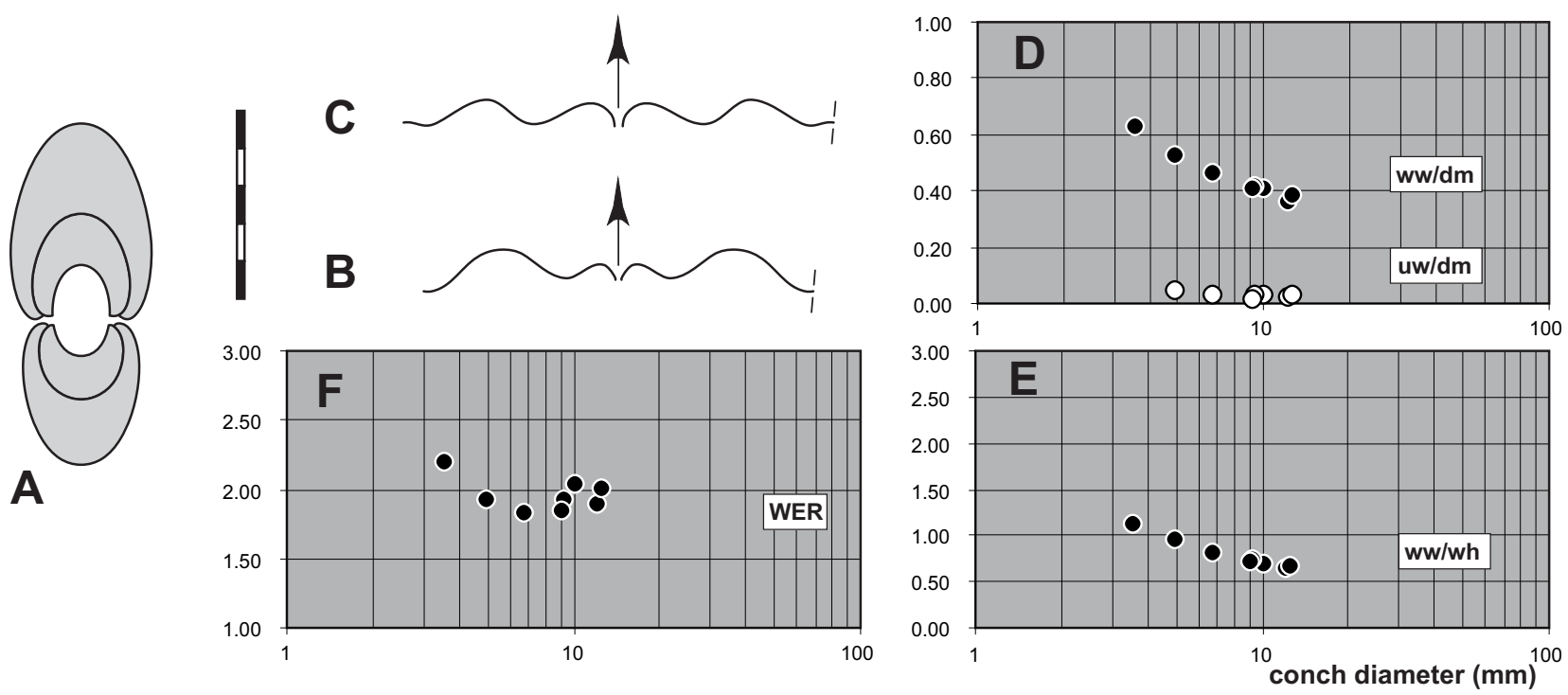

Figure 60. Lobotornoceras bensaidi n. sp. from Hassi Nebech; A. Cross-section of paratype MB.C.22102.2, $\times 4$; B. Suture of paratype MB.C.22102.2 at $5.2 \mathrm{~mm}$ wh, $\times 4$; C. Suture of paratype MB.C.22102.3 at $5.9 \mathrm{~mm}$ wh, $\times 4$; D-F. Ontogenetic development of ww/dm, uw/dm, ww/wh, and WER of all available specimens.
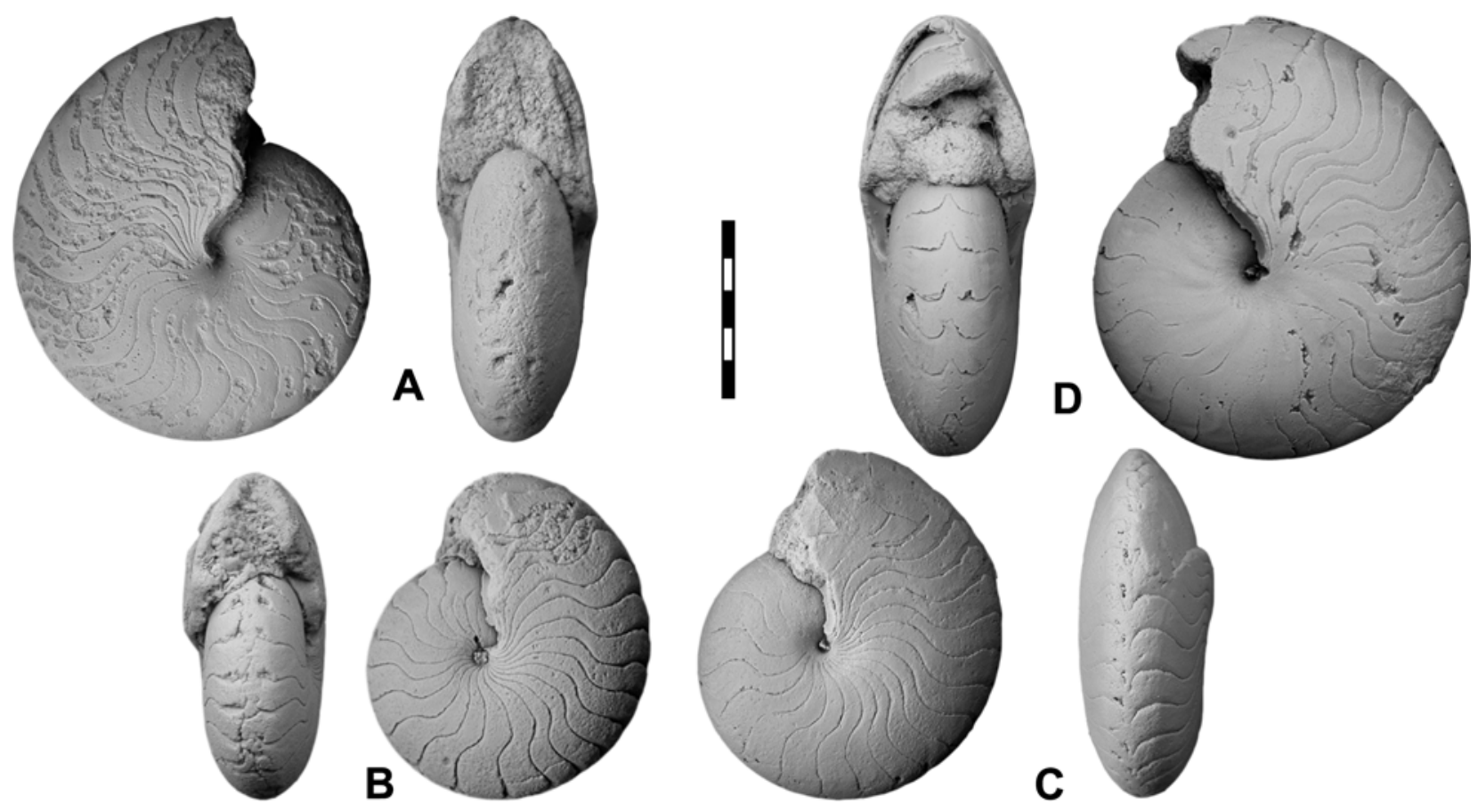

Figure 61. Lobotornoceras bensaidi n. sp. from Hassi Nebech; A. Paratype STIPB-Bensaïd-44 (old: GPI Bo 44), original of Tornoceras frechi of Bensaïd (1974: pl. 5, figs 4, 4a); B. Paratype MB.C.22102.2 (before sectioning); C. Paratype MB.C.22102.3; D. Holotype MB.C.22102.1; all $\times 4$. 
Table 57. Conch ontogeny (Figs 60A, D-F) of Lobotornoceras bensaidi $\mathrm{n}$. sp.

\begin{tabular}{llll}
\hline $\mathrm{dm}$ & conch shape & whorl cross-section shape & whorl expansion \\
\hline $5 \mathrm{~mm}$ & thickly discoidal; involute & weakly compressed; very strongly embracing & moderate \\
& $($ ww/dm $\sim 0.53 ;$ uw/dm $\sim 0.05)$ & $($ ww/wh $\sim 0.95 ;$ IZR $\sim 0.50)$ & $($ WER $\sim 1.95)$ \\
$10 \mathrm{~mm}$ & thinly discoidal; involute & weakly compressed; very strongly embracing & high \\
& $($ ww/dm $\sim 0.40 ;$ uw/dm $\sim 0.04)$ & $($ ww/wh $=0.70-0.75 ;$ IZR $\sim 0.50)$ & $($ WER $\sim 2.00)$
\end{tabular}

Table. 58. Suture formula (Figs 60B, C), conch characteristics, and ornament of Lobotornocers bensaidi $\mathrm{n}$. sp.

$E L_{1 e}: L_{1 i} l$

E lobe short; A lobe broadly rounded, shallow; EA and AL saddles low; L lobe divided by small, low saddle at the umbilicus

venter narrowly rounded; umbilicus closed

no ribs; no ventrolateral furrows; no internal constrictions

growth lines strongly biconvex, bundled

Diagnosis. Small, earliest stages currently unknown; conch from $5 \mathrm{~mm}$ dm strictly involute, compressed, gradually becomming thinly discoidal (ww/dm decreasing from $>0.60$ to ca. 0.40 ), with gently rounded flanks and narrowly rounded venter, WER rising from ca. 1.80 to ca. 2.00 at $>10 \mathrm{~mm} \mathrm{dm}$, no mould constrictions. Sutures with small L lobe, asymmetric, wide and low AL saddle, widely rounded, shallow A lobe, low, asymmetric ventrolateral saddle and small, slightly diverging E lobe. The biconvex growth lines are best visible on the holotype.

\section{Subfamily Falcitornoceratinae Becker, 1993}

Diagnosis. Embryonic first whorl and first post-embryonic whorl depressed, subsequent whorls with completely closed umbilicus, weakly to strongly compressed, WER moderately high to high, venter rounded or oxyconic; growth lines strongly biconvex in early stages, weakly or strongly biconvex at maturity, often forming distinctive lirae or ribs, with shallow or strong ventrolateral furrows, at least in early whorls, with or without internal shell thickenings. L lobe undivided or divided, A lobe wide and shallow or deep, lingulate to asymmetrically pointed, external lobe small. Suture formulae: EALI, EAL $L_{e}: \mathrm{L}_{\mathrm{i}} \mathrm{I}$ or $\mathrm{EAL}_{1 \mathrm{e}} \mathrm{L}_{2} \mathrm{~L}_{1 \mathrm{i}} \mathrm{I}$.

\section{Nebechoceras n. gen.}

Derivation of name. After the type locality Hassi Nebech.

Type species. Nebechoceras excentricum $\mathrm{n}$. sp.

Diagnosis: First two whorls depressed and openly umbilicate, subsequently increasingly compressed, with high WER, weak ventrolateral furrows, and rounded venter; umbilicus closed at maturity, from the 6th whorl on with a sudden umbilical opening of internal moulds, probably caused by a shell callus; growth lines strongly biconvex, with prominent ventrolateral salient. Suture line with asymmetrically rounded adventive lobe and low ventrolateral saddle. Suture formula EALI.

Included species. Only the type species.

Stratigraphic range and geographic distribution. Type locality and horizon.

\section{Nebechoceras excentricum n. sp.}

\section{Figures 62, 63}

Derivation of name. After the sudden, eccentric umbilical opening on internal moulds of mature whorls.

Holotype. MB.C.22103.1 (Figs 62K \& 63A).

Table 59. Conch ontogeny (Figs 62A-I, M-O) of Nebechoceras excentricum n. gen. n. sp.

\begin{tabular}{|c|c|c|c|}
\hline $\mathrm{dm}$ & conch shape & whorl cross-section shape & whorl expansion \\
\hline $2 \mathrm{~mm}$ & $\begin{array}{l}\text { thickly discoidal; subinvolute } \\
\text { (ww/dm =0.55-0.60; uw/dm 0.25) }\end{array}$ & $\begin{array}{l}\text { weakly depressed; strongly embracing } \\
\text { (ww/wh } \sim 1.10 ; \text { IZR }=0.40-0.45 \text { ) }\end{array}$ & $\begin{array}{l}\text { high } \\
(\text { WER }=2.00-2.10)\end{array}$ \\
\hline $5 \mathrm{~mm}$ & $\begin{array}{l}\text { thickly discoidal; involute } \\
(\mathrm{ww} / \mathrm{dm}=0.50-0.55 ; \mathrm{uw} / \mathrm{dm}=0.05-0.10)\end{array}$ & $\begin{array}{l}\text { weakly compressed; very strongly embracing } \\
\text { (ww/wh } \sim 0.95 ; \text { IZR } \sim 0.50 \text { ) }\end{array}$ & $\begin{array}{l}\text { moderate } \\
(\text { WER } \sim 2.00)\end{array}$ \\
\hline $10 \mathrm{~mm}$ & $\begin{array}{l}\text { thinly to thickly discoidal; involute } \\
\text { (ww/dm }=0.45-0.55 ; \text { uw/dm } \sim 0.05 \text { ) }\end{array}$ & $\begin{array}{l}\text { weakly compressed; very strongly embracing } \\
(\mathrm{w} w / \mathrm{wh}=0.80-0.90 ; \mathrm{IZR}=0.45-0.50)\end{array}$ & $\begin{array}{l}\text { high } \\
(\text { WER }=2.00-2.20)\end{array}$ \\
\hline $15 \mathrm{~mm}$ & $\begin{array}{l}\text { thinly discoidal; involute } \\
\text { (ww/dm } \sim 0.45 ; \text { uw/dm } \sim 0.05)\end{array}$ & $\begin{array}{l}\text { weakly compressed; very strongly embracing } \\
(\mathrm{w} w / w h=0.70-0.80 ; \mathrm{IZR}=0.45-0.50)\end{array}$ & $\begin{array}{l}\text { high } \\
(\text { WER }=2.00-2.20)\end{array}$ \\
\hline
\end{tabular}


Table 60. Suture formula (Figs 62A, J-L), conch characteristics, and ornament of Nebechoceras excentricum n. gen. n. sp.

EALI

E lobe parallel-sided or funnel-shaped, moderately deep; A lobe moderately deep, asymmetric, broadly rounded; I lobe deep, narrow conch tegoid; venter rounded; umbilical margin rounded or flattened; sudden umbilical uncoiling at about $20 \mathrm{~mm} \mathrm{dm}$ with (Morphotype II) or without (Morphotype I) ventrolateral furrows; no ribs; no internal constrictions growth lines biconvex, lirate, with high ventrolateral projection

Type locality and horizon. Hassi Nebech, Section 2, SE Tafilalt, main collecting level, probably Taouzites taouzensis Zone (late Givetian). Material. More than 50 specimens up to $25 \mathrm{~mm}$ conch diameter, including MB.C.22103.1-MB.C.22103.22.

Diagnosis. Embryonic whorl subinvolute and depressed, from second whorl on with constant absolute umbilical width but rapidly decreasing uw $/ \mathrm{dm}$ values, at ca. $20 \mathrm{~mm} \mathrm{dm}$ with sudden opening of the umbilicus on internal moulds; cross-section tegoid, compressed from 5-6 mm dm,

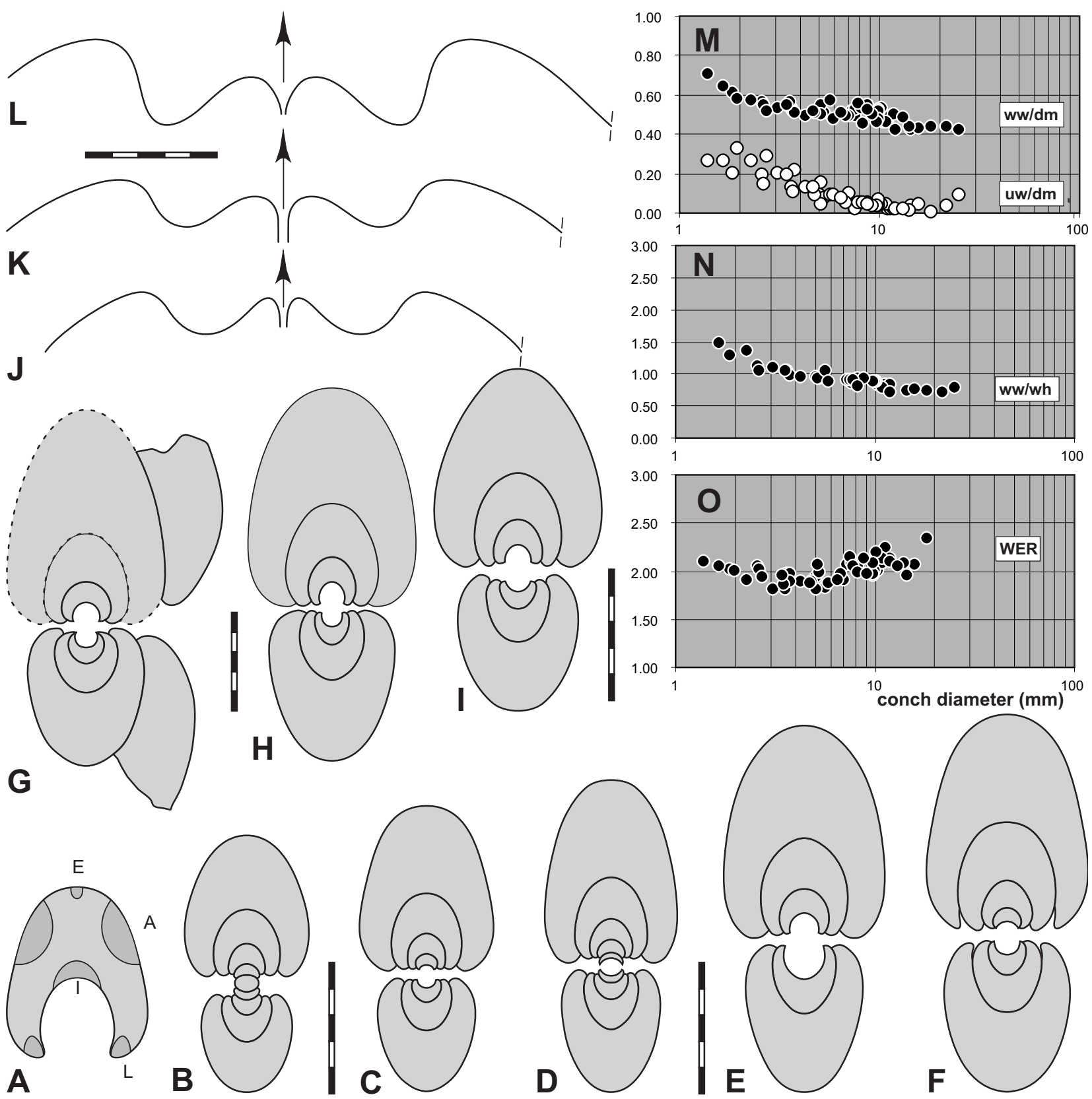

Figure 62. Nebechoceras excentricum n. gen. n. sp. from Hassi Nebech; Morphotype I: C, H, Morphotype II: A, B, D-G, I; A. Septal face of paratype MB.C.22103.2, $\times 4$; B-I. Cross-sections; B. Paratype MB.C.22103.3, $\times 4$; C. Paratype MB.C.22103.4, $\times$ 4; D. Paratype MB.C.22103.5, $\times$ 4; E. Paratype MB.C.22103.6, $\times 4$; F. Paratype MB.C.22103.7, $\times 4$; G. Paratype MB.C.22103.8, $\times 3$; H. Paratype MB.C.22103.9, $\times 3$; I. Paratype MB.C.22103.10, $\times 4$; J-L. Sutures, all $\times 4$; J. Paratype MB.C.22103.18 at $7 \mathrm{~mm}$ wh; K. Holotype MB.C.22103.1 at $9 \mathrm{~mm}$ wh; L. Paratype MB.C.22103.9 at $10 \mathrm{~mm}$ wh; M-O. Ontogenetic development of ww/ $\mathrm{dm}, \mathrm{uw} / \mathrm{dm}, \mathrm{ww} / \mathrm{wh}$, and WER. 

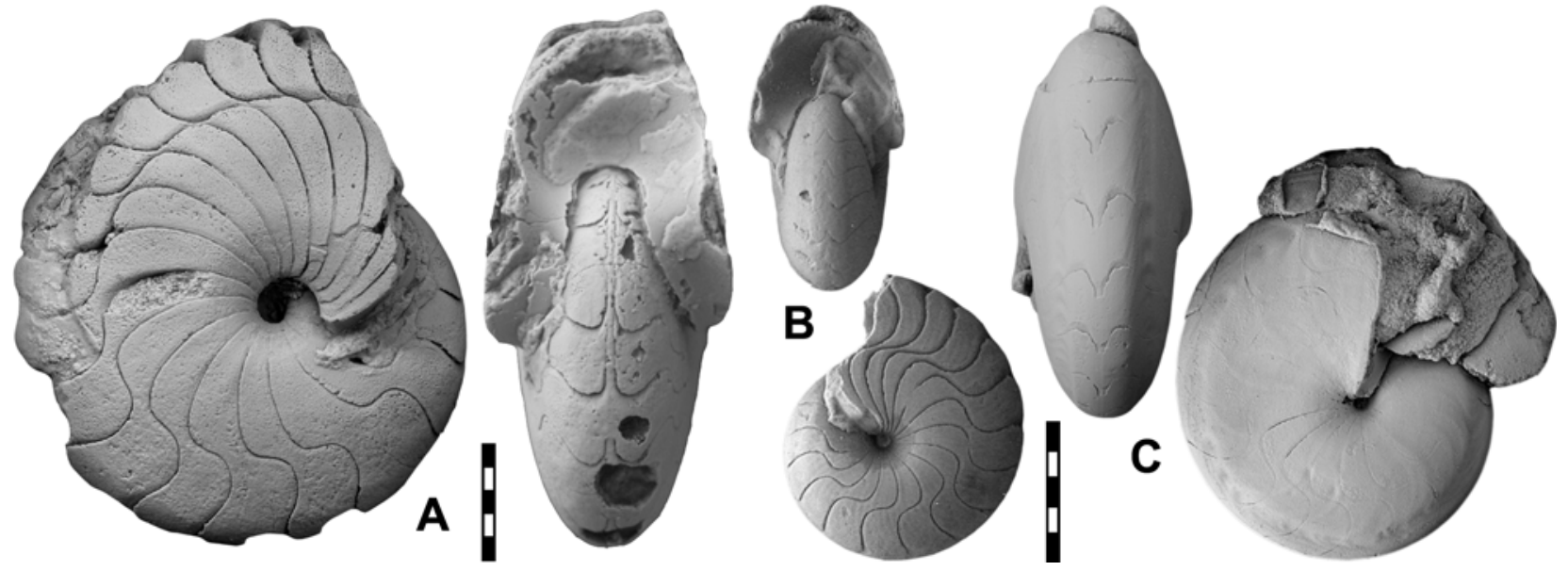

Figure 63. Nebechoceras excentricum n. gen. n. sp. from Hassi Nebech; A. Holotype MB.C.22103.1, Morphotype I, $\times 2.5$; B. Paratype MB.C.22103.11, Morphotype I, $\times 3$; C. Paratype MB.C.22103.12, Morphotype II, $\times 3$.

with ww $/ \mathrm{dm}=0.4$ at ca. $25 \mathrm{~mm} \mathrm{dm}$, and high WER, but moderate WER until ca. $7 \mathrm{~mm} \mathrm{dm}$. Ornament consists of biconvex growth lirae with high ventrolateral salient. Sutures with small, divergent E lobe, low, asymmetric ventrolateral saddle, rounded A lobe, asymmetrically arched, high dorsolateral saddle, and moderately deep, parallel-sided I lobe.

\section{Phoenixites Becker, 1993}

Type species. Tornoceras frechi Wedekind, 1918.

Diagnosis. See Becker (1993).

\section{Phoenixites lenticulus n. sp.}

Figures 64, 65

Derivation of name. After the lenticular conch shape.

Holotype. MB.C.22104.1 (Figs 64D \& 65B).

Type locality and horizon. Hassi Nebech, Section 2, SE Tafilalt, main collecting level, probably Taouzites taouzensis Zone (late Givetian). Material. 30 specimens up to $15 \mathrm{~mm}$ conch diameter, including MB.C.22104.1-MB.C.22104.16.

Table 61. Conch ontogeny (Figs 64A-C, G-I) of Phoenixites lenticulus n. sp.

\begin{tabular}{|c|c|c|c|}
\hline $\mathrm{dm}$ & conch shape & whorl cross-section shape & whorl expansion \\
\hline $2 \mathrm{~mm}$ & $\begin{array}{l}\text { thinly pachyconic; subinvolute } \\
\text { (ww/dm } \sim 0.65 ; \text { uw/dm } \sim 0.15 \text { ) }\end{array}$ & $\begin{array}{l}\text { weakly depressed; very strongly embracing } \\
\text { (ww/wh } \sim 1.20 \text {; IZR } \sim 0.50 \text { ) }\end{array}$ & moderate \\
\hline $5 \mathrm{~mm}$ & $\begin{array}{l}\text { thickly discoidal; involute } \\
\text { (ww/dm } \sim 0.55 ; \text { uw/dm } \sim 0.10 \text { ) }\end{array}$ & $\begin{array}{l}\text { weakly compressed; very strongly embracing } \\
\text { (ww/wh } \sim 1.00 ; \text { IZR } \sim 0.50 \text { ) }\end{array}$ & $\begin{array}{l}\text { moderate } \\
(\text { WER } \sim 1.90)\end{array}$ \\
\hline $8 \mathrm{~mm}$ & $\begin{array}{l}\text { thickly discoidal; involute } \\
(\mathrm{ww} / \mathrm{dm} \sim 0.55 ; \text { uw/dm } \sim 0.05)\end{array}$ & $\begin{array}{l}\text { weakly compressed; very strongly embracing } \\
\text { (ww/wh } \sim 0.90 ; \text { IZR }=0.45-0.50 \text { ) }\end{array}$ & $\begin{array}{l}\text { moderate to high } \\
(\text { WER }=1.90-2.10)\end{array}$ \\
\hline $13 \mathrm{~mm}$ & $\begin{array}{l}\text { thickly discoidal; involute } \\
\text { (ww/dm } \sim 0.47 ; \text { uw/dm } \sim 0.03 \text { ) }\end{array}$ & $\begin{array}{l}\text { weakly compressed; very strongly embracing } \\
\text { (ww/wh } \sim 0.80 ; \text { IZR } \sim 0.48)\end{array}$ & $\begin{array}{l}\text { high } \\
\text { (WER 2.10) }\end{array}$ \\
\hline
\end{tabular}

Table 62. Suture formula (Figs 64A, D-F), conch characteristics, and ornament of Phoenixites lenticulus n. sp.

\section{EALI}

E lobe short, funnel-shaped; A lobe shallow to moderately deep, narrow, asymmetrical; EA saddle lower than broadly rounded AL saddle; I lobe deep, narrow

venter narrowly rounded; umbilicus closed

striking ventrolateral grooves; no ribs; no mould constrictions

growth lines strongly biconvex, with low dorsolateral salient and high ventrolateral projection within the grooves 


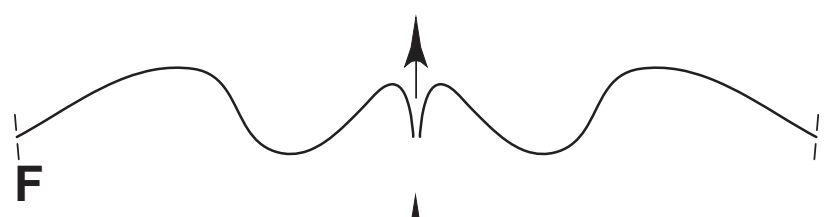

$\mathbf{F}$

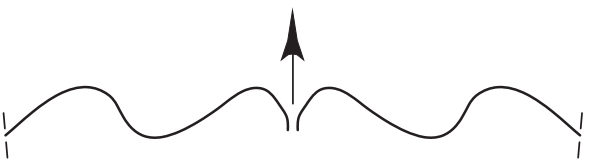

E

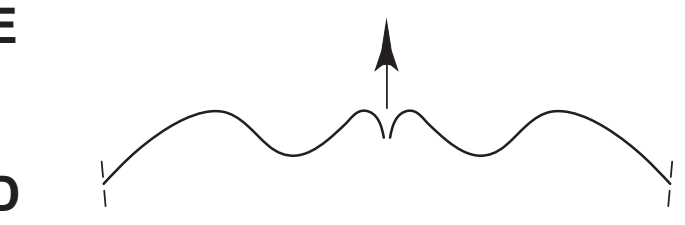

A

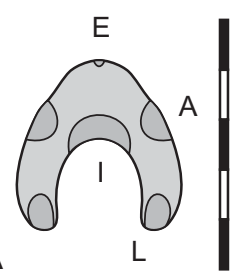

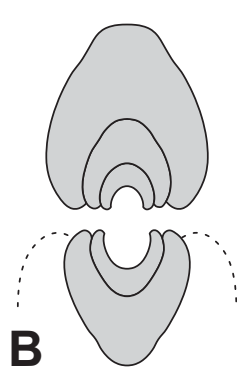

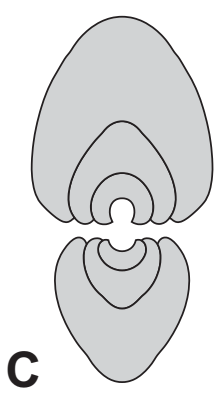

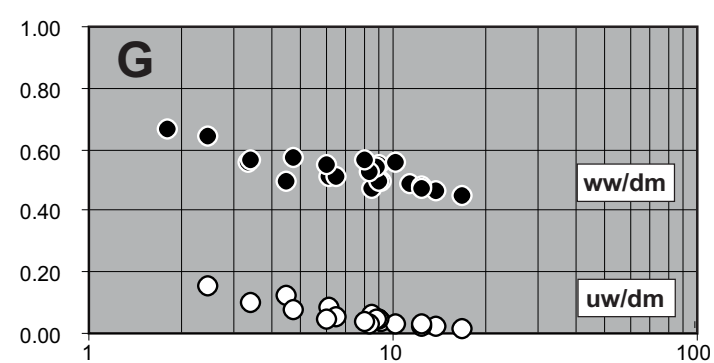
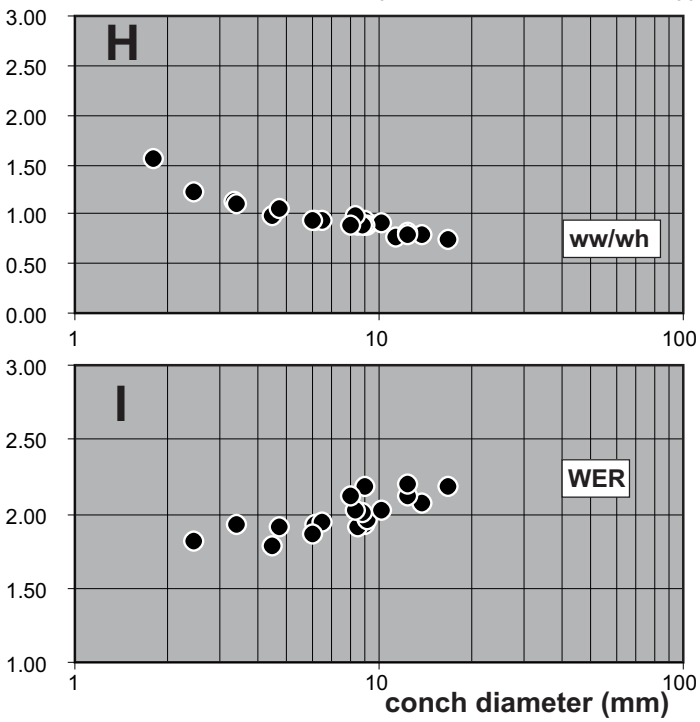

Figure 64. Phoenixites lenticulus n. sp. from Hassi Nebech; A. Septal face of paratype MB.C.22104.2, $\times 4$; B. Cross-section of paratype MB.C.22104.3, $\times 4$; C. Cross-section of paratype MB.C.22104.4, $\times 4$; D-F. Sutures, all $\times 4$; D. Holotype MB.C.22104.1 at $5.8 \mathrm{~mm}$ wh; E. Paratype MB.C.22104.5 at $6 \mathrm{~mm}$ wh; F. Paratype MB.C.22104.6 at $9.3 \mathrm{~mm}$ wh; G-I. Ontogenetic development of ww/dm, uw/dm, ww/wh, and WER.
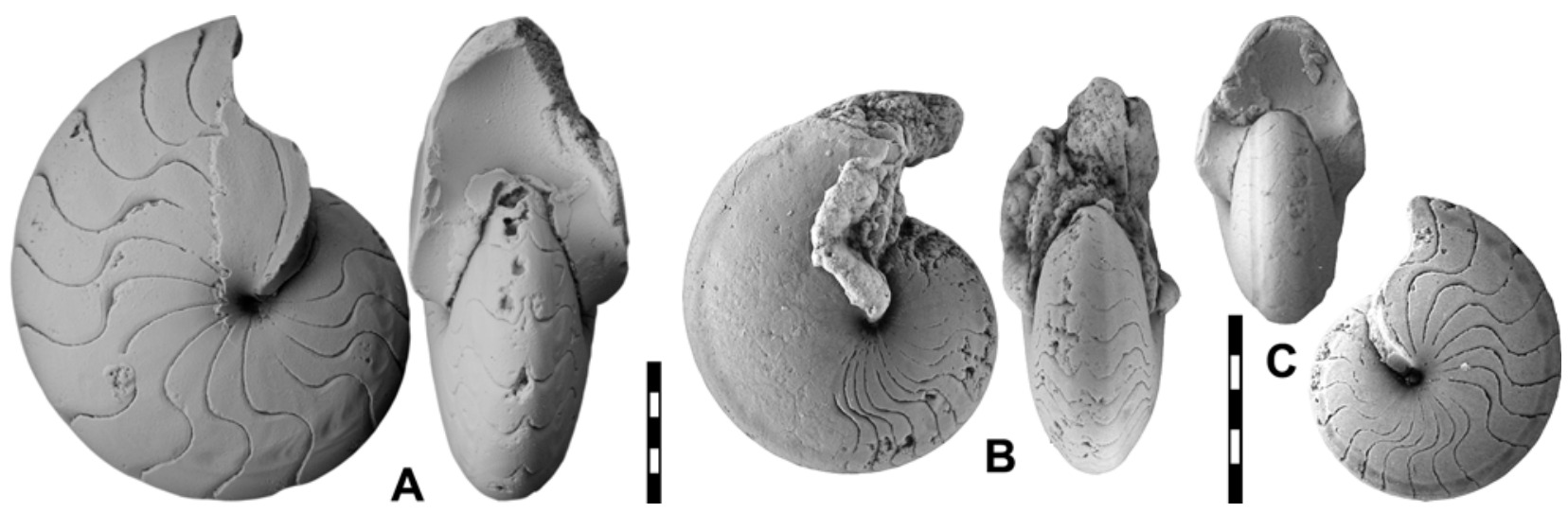

Figure 65. Phoenixites lenticulus n. sp. from Hassi Nebech; A. Paratype MB.C.22104.7, $\times 3$; B. Holotype MB.C.22104.1, $\times 3$; C. Paratype MB.C.22104.8, $\times 4$.

Diagnosis. Embryonic whorl subinvolute and depressed, from second whorl on with constant absolute umbilical width but strongly decreasing uw $/ \mathrm{dm}$ ratios $(<0.05$ from ca. $5 \mathrm{~mm} \mathrm{dm})$, increasingly compressed $(w w / w h=0.80$ at $13-14 \mathrm{~mm} \mathrm{dm}, w w / d m=c a .0 .46$ at $15 \mathrm{~mm} \mathrm{dm})$ and with rising WER (high from ca. $8 \mathrm{~mm} \mathrm{dm}$ ); cross-section lenticular to subtrapezoidal, with rounded, strongly converging flanks and narrow, flatly rounded venter; growth lines strongly biconvex, with projecting, narrow ventrolateral salient lying in shallow or marked spiral groves, at least until $4 \mathrm{~mm}$ wh. Sutures with divergent (early stages) to subparallel (mature stages) I lobe, moderately low, asymmetric dorsolateral saddle, widely rounded, asymmetric A lobe on outer flanks, even lower, asymmetrical, narrow ventrolateral saddle, and small, divergent E lobe. 


\section{Acknowledgements}

This paper is devoted to M. Bensaid (Rabat) in honour of his significant contribution to research on Moroccan pharciceratids. Early parts of the field work, including measurements of sections, were conducted jointly with the late M. R. House. Z. S. Aboussalam (Münster) also joined us during several field campaigns and assisted during collecting. Work permits and fossil export licences were provided over several years by M. Bensaïd and M. Dahmani (Ministére de l'Energie et des Mines, Rabat) and, in more recent years, together with general support, by our principal Moroccan collaborator, A. El Hassani (Institut Scientifique, Rabat). R. Mapes (Athens/Ohio) kindly made RTB his large purchased Hassi Nebech collection available for examination during a brief visit to his institute. The late W. Haas, S. I. Kaiser, and G. Heumann (all Steinmann Institute, University Bonn) enabled at different times the examination and partial loan of the Bensaï Collection at the Goldfuß Museum. D. Korn (Museum für Naturkunde, Berlin) enabled the access and loan of German late Givetian ammonoids. F. Geller-Grimm and D. Heidelberger, (Landesmuseum Wiesbaden), U. Jansen (Forschungsinstitut Senckenberg, Frankfurt a.M.) loaned other specimens from the southern Rhenish Massive. T. Fährenkemper (Münster) produced the section log and global ammonoid distribution map. We are very thankful for the detailed reviews of K. De Baets, Zürich / Bristol and D. M. Work, Augusta.

\section{References}

Aboussalam, Z. S. 2003. Das „Taghanic-Event“ im höheren MittelDevon von West-Europa und Marokko. - Münstersche Forschungen zur Geologie und Paläontologie 97: 1-332.

Aboussalam, Z. S. \& Becker, R. T. 2007. New Upper Givetian to basal Frasnian conodont faunas from the Tafilalt (Anti-Atlas, southern Morocco). - Geological Quarterly 51 (4): 345-374.

Aboussalam, Z. S. \& Becker, R. T. 2011. The global Taghanic Biocrisis (Givetian) in the eastern Anti-Atlas, Morocco. - Palaeogeography, Palaeoclimatology, Palaeoecology 304: 136-164.

Arthaber, G. von 1911. Die Trias von Albanien. - Beiträge zur Paläontologie und Geologie Österreich-Ungarns und des Orients 24: 169-277.

Becker, R. T. 1993. Stratigraphische Gliederung und AmmnonoideenFaunen im Nehdenium (Oberdevon II) von Europa und Nord-Afrika. - Courier Forschungsinstitut Senckenberg 155: 1-405.

Becker, R. T. \& House, M. R. 1993. New early Upper Devonian (Frasnian) goniatite genera and the evolution of the "Gephurocerataceae". - Berliner geowissenschaftliche Abhandlungen, Reihe E 9: 111-133.

Becker, R. T. \& House, M. R. 2000a. Late Givetian and Frasnian ammonoid succession at Bou Tchrafine (Anti-Atlas, Southern Morocco). - Notes et Mémoires du Service Géologique 399: 27-36.

Becker, R. T. \& House, M. R. 2000b. Devonian ammonoid zones and their correlation with established series and stage boundaries. Courier Forschungsinstitut Senckenberg 220: 113-151.

Belka, Z., Klug, C., Kaufmann, B., Korn, D., Döring, S., Feist, R. \& Wendt, J. 1999. Devonian conodont and ammonoid succession of the eastern Tafilalt (Ouidane Chebbi section), Anti-Atlas, Morocco. - Acta Geologica Polonica 49 (1): 1-23.

Bensaïd, M. 1974. Etude sur des Goniatites a la limite du Devonien Moyen et Supérieur, du Sud Marocain. - Notes de Service Carte géologique du Maroc 36 (264): 81-140.

Bensaï,, M., Bultynck, P., Sartenaer, P., Walliser, O. H. \& Ziegler, W. 1985. The Givetian-Frasnian Boundary in pre-Sahara Morocco. Courier Forschungsinstitut Senckenberg 75: 287-300

Bockwinkel, J., Becker, R. T. \& Ebbighausen, V. 2009. Upper Givetian ammonoids from Dar Kaoua (Tafilalt, SE Anti-Atlas, Morocco). - Berliner paläobiologische Abhandlungen 10: 61-128.
Bogoslovsky, B. I., Librovitch, L. S. \& Ruzhencev, V. E. 1962. Nadotryad Ammonoidea. Sistematicheskaya chast'. In Yu. A. Orlov \& V. E. Ruzhencev (eds) Osnovy Paleontologii 5, Mollyuski: Golovonogie 1; Akademiya Nauk SSSR, Moskva, pp.: 334425 .

Clariond, L. 1934. A propos d'une coupe de la region d'Erfoud. Compte Rendu somnaire de la Société Géologique de France 1934: 223-224.

Frech, F. 1888. Geologie der Umgebung von Haiger bei Dillenburg (Nassau). - Abhandlungen der Königlich Preussischen Geologischen Landesanstalt 8 (3): 223-258

Frech, F. 1897. Lethaea geognostica oder Beschreibung und Abbildung der für die Gebirgs-Formationen bezeichnensten Versteinerungen. I. Theil. Lethaea paleozoica, 2. Band IV: 1-283.

Frech, F. 1902. Über devonische Ammoneen. - Beiträge zur Paläontologie Österreich-Ungarns und des Orients 14: 27-112.

Göddertz, B. 1987. Devonische Goniatiten aus SW-Algerien und ihre stratigraphische Einordnung in die Conodonten-Abfolge. - Palaeontographica, Abt. A 197 (4-6): 127-220.

Gouwy, S., Haydukiewicz, J. \& Bultynck, P. 2007. Conodont-based graphic correlation of upper Givetian-Frasnian sections of the Eastern Anti-Atlas (Morocco). - Geological Quarterly 51 (4): 375-392.

Heckel, P. H. \& Witzke, B. J. 1979. Devonian world palaeogeography determined from distribution of carbonates and related lithic palaeoclimatic indicators. - Special Papers in Palaeontology 23: 99-123.

Hollard, H. 1981. Tableaux de corrélations du Silurien et du Dévonien de l'Anti-Atlas. - Notes du Service Geologiques du Maroc 308: 23.

House, M. R. 1985. Correlation of mid-Palaeozoic ammonoid evolutionary events with global sedimentary perturbations. - Nature 313 (5997): 17-22.

House, M. R. \& Ziegler, W. 1977. The Goniatite and Conodont sequences in the early Upper Devonian at Adorf, Germany. - Geologica et Palaeontologica 11: 69-108.

House, M. R., Kirchgasser, W. T., Price, J. D. \& Wade, G. 1985. Goniatites from Frasnian (Upper Devonian) and adjacent strata of the Montagne Noire. - Hercynica 1 (1): 1-21.

Hyatt, A. 1884. Genera of fossil cephalopods. - Proceedings of the Boston Society of Natural History 22: 273-338 [pp. 253-272 published in 1883]

Hyatt, A. 1900. Cephalopoda. In Zittel, K. A. v. (ed., transl \& ed. by C. R. Eastman). Text-book of palaeontology, volume 1, 1st edition. McMillan, London, New York: pp. 502-604.

Kaufmann, B. 1998. Facies, stratigraphy and diagenesis of Middle Devonian reef- and mud-mounds in the Mader (eastern Anti-Atlas, Morocco). - Acta Geologica Polonica 48 (1): 43-106.

Kennedy, W. J. \& Cobban, W. A. 1990. Cenomanian micromorphic ammonites from the Western Interior of the USA. - Palaeontology 33 (2): 379-422.

Klug, C. \& Korn, D. 2004. The origin of ammonoid locomotion. Acta Palaeontologica Polonica, 49 (2): 235-242.

Korn, D. 1998. Phylogenie unter- und mitteldevonischer Ammonoideen. - Terra Nostra 98/3, Geo-Berlin '98, Programm und Zusammenfassungen: V182-V183.

Korn, D. 2001. Morphometric evolution and phylogeny of Palaeozoic ammonoids. Early and Middle Devonian. - Acta Geologica Polonica 51 (3): 193-215.

Korn, D. 2010. A key for the description of Palaeozoic ammonoids. Fossil Record 13 (1): 5-12.

Korn, D. \& Klug, C. 2002. Ammoneae Devonicae. - Fossilium Catalogus Animalia 138: 1-375.

Landman, N. H. 1988. Heterochrony in ammonites. In McKinney, M. L. (ed.). Heterochrony in Evolution. Plenum, New York: 159182.

Marchand, D., Courville, P., Bonnet, A., Rossi, J. \& Scouflaire, Q. 2002. Very Small Ammonites (Micromorphs) from Lower Oxfor- 
dian Marls (Mariae Zone). - Abhandlungen der Geologischen Bundesanstalt 57: 467-478.

Monnet, C., De Baets, K. \& Klug, C. 2011. Parallel evolution controlled by adaptation and covariation in ammonoid cephalopods. BMC Evolutionary Biology 11: 115. DOI: 10.1186/1471-2148$11-115$

Montesinos, R. A \& Henn, A. H. 1986. La fauna de Pharciceras (Ammonoidea) de la Formacion Cardano (Dominio Palentino, Cordillera Cantabrica, NO de Espana). - Trabajos de Geologia 16: $61-76$.

Ogg, J. G., Ogg, G. \& Gradstein, F. M. 2008. The Concise Geological Time Scale. Cambrige University Press, Cambridge: 177 pp.

Petter, G., 1959. Goniatites Dévoniennes du Sahara. - Publications du Service de la Carte Géologique de l'Algérie, Nouvelle Série, Paléontologie 2: 1-313.

Ruzhencev, V. E. 1957. Filogeneticheskaya sistema paleozoyskikh ammonoidey. - Byulleten Moskovskogo obshchestva ispytately prirody, novaya seriya, otdel geologicheskiy 31 (2): 49-64.

Sandberger, G. \& Sandberger, F. 1850-1856. Die Versteinerungen des Rheinischen Schichtensystems in Nassau. Wiesbaden: 564 [pp. 172 and pls. 1-8 published in 1850].

Schindewolf, O. H. 1936. Neue Gattungen tiefoberdevonischer Goniatiten. - Zeitschrift der Deutschen Geologischen Gesellschaft 88: 689-691.

Schindewolf, O. H. 1940. Konvergenzen bei Korallen und bei Ammoneen. - Fortschritte der Geologie und Palaeontologie 12 (41): 389-492.
Schindewolf, O. H. 1955. Zur Taxionomie und Nomenklatur der Clymenien. Ein Epilog. - Neues Jahrbuch für Geologie und Paläontologie, Monatshefte, 1955: 417-429.

Skelton, P. (ed.) 1993. Evolution. A Biological and Palaeontological Approach. Addison-Wesley Publishing Company, Wokingham, Reading etc.: $1064 \mathrm{pp}$.

Stegemann, T. 2005. Taxonomie und Phylogenie von Phacopiden (Trilobita) des höheren Givetiums (Mitteldevon). - Münstersche Forschungen zur Geologie und Paläontologie 100: 21-33.

Steininger, J. 1849. Die Versteinerungen des Uebergangsgebirges der Eifel. Trier: $34 \mathrm{pp}$.

Termier, H. 1929. Sur le Dévonien du Tafilalt. - Compte Rendu de l'Academie des Sciences 1929: 158-160.

Termier, H. \& Termier, G. 1950. Paléontologie Marocaine. II. Invertébres de l'ère Primaire. Fascicule III. Mollusques. - Notes et Mémoires du Service Géologique, Rabat 78: 1-246.

Wedekind, R. 1918. Die Genera der Palaeoammonoidea (Goniatiten). - Palaeontographica 62: 85-184.

Wendt, J. 1988. Facies pattern and paleogeography of the Middle and Late Devonian in the eastern Anti-Atlas (Morocco). In McMillan, N. J., Embry, A. F. \& Glass, D. J. (eds). Devonian of the World, Volume I: Regional Syntheses. Canadian Society of Petroleum Geologists, Memoir 14 (I): 467-480.

Wendt, J., Aigner, T. \& Neugebauer, J. 1984. Cephalopod limestone deposition on a shallow pelagic ridge: the Tafilalt Platform (upper Devonian, eastern Anti-Atlas, Morocco). - Sedimentology 31: 601-625. 